\title{
Evaluation of ischemic injury in donor kidneys : an experimental study
}

Citation for published version (APA):

Maessen, J. G. (1988). Evaluation of ischemic injury in donor kidneys : an experimental study. [Doctoral Thesis, Maastricht University]. Rijksuniversiteit Limburg. https://doi.org/10.26481/dis.19880916jm

Document status and date:

Published: 01/01/1988

DOI:

10.26481/dis.19880916jm

Document Version:

Publisher's PDF, also known as Version of record

\section{Please check the document version of this publication:}

- A submitted manuscript is the version of the article upon submission and before peer-review. There can be important differences between the submitted version and the official published version of record.

People interested in the research are advised to contact the author for the final version of the publication, or visit the DOI to the publisher's website.

- The final author version and the galley proof are versions of the publication after peer review.

- The final published version features the final layout of the paper including the volume, issue and page numbers.

Link to publication

\footnotetext{
General rights rights.

- You may freely distribute the URL identifying the publication in the public portal. please follow below link for the End User Agreement:

www.umlib.nl/taverne-license

Take down policy

If you believe that this document breaches copyright please contact us at:

repository@maastrichtuniversity.nl

providing details and we will investigate your claim.
}

Copyright and moral rights for the publications made accessible in the public portal are retained by the authors and/or other copyright owners and it is a condition of accessing publications that users recognise and abide by the legal requirements associated with these

- Users may download and print one copy of any publication from the public portal for the purpose of private study or research.

- You may not further distribute the material or use it for any profit-making activity or commercial gain

If the publication is distributed under the terms of Article $25 \mathrm{fa}$ of the Dutch Copyright Act, indicated by the "Taverne" license above, 
Evaluation of ischemic injury in donor kidneys

an experimental study 



\title{
Evaluation of ischemic injury in donor kidneys
}

\author{
an experimental study
}

Proefschrift

ter verkrijging van de graad van doctor aan de Rijksuniversiteit Limburg te Maastricht, op gezag van de Rector Magnificus, Prof. dr. F.I.M. Bonke, volgens het besluit van het College van Dekanen, in het openbaar te verdedigen op vrijdag 16 september 1988 om 14.00 uur

$$
\text { door }
$$

Josephus Gregorius Maessen geboren te Roermond in 1960 
Promotores:

Prof.dr. G. Kootstra

Prof.dr. G.J. van der Vusse

Co-promotor:

Dr.W.Th.Hermens

Beoordelingscommissie:

Prof. P.J. Hoedemaeker

Dr. J.P. van Hooff

Dr. J.W. de Jong

Prof.dr. O.C.K.M. Penn (voorzitter)

Prof.dr. R.S. Reneman

Dr. M.J.H. Slooff

Prof.dr. H.A.J. Struyker Boudier

Het onderzoek in dit proefschrift beschreven werd financieel mogelijk gemaakt door steun van de Nierstichting Nederland. 
Aan mijn ouders Voor Jet 


\section{CONTENTS}

Preface

PART I

CHAPTER 1

Integrity of the cellular energy metabolism during ischemia and reperfusion introduction 4

viability

purine nucleotides

renal adenine nucleotide content

normothermic ischemia

reperfusion following ischemia

hypothermia

cooling by flushing

cold storage

viability assessment

concluding remarks

\section{CHAPTER 2}

Quantitation of ischemic, structural renal cell damage

introduction

enzymes as markers of tissue damage $\quad 32$

estimation of infarct size 33

transport of enzymes from kidney to plasma or urine 34

changes in tissue enzyme activity 34

enzyme activity following ischemic renal injury 35

cumulative amount of plasma enzyme activity 36

tissue enzyme loss and plasma enzyme activity 37

\section{CHAPTER 3}

Potentiation of ischemic injury by inflammatory mediators

introduction

reperfusion injury

inflammatory response

modulation of the inflammatory response

future prospects 


\section{CHAPTER 4}

Determination of warm ischemia time during donornephrectomy

\section{CHAPTER 5}

Inability to maintain adenine nucleotide levels by cold storage in ischemically damaged and control kidneys

\section{CHAPTER 6}

New approach to preservation of non-heart beating donor kidneys: total body cooling

\section{CHAPTER 7}

Potentiation of ischemic injury by hypothermia in donor kidneys: role of the energy metabolism

\section{CHAPTER 8}

Assessment of nucleotides, nucleosides, and oxypurines in human donor kidneys

\section{CHAPTER 9}

The stunned kidney: postischemic renal failure and adenine nucleotide homeostasis

\section{CHAPTER 10}

Beneficial effect of intermediate normothermic perfusion during cold storage of ischemically injured kidneys

\section{CHAPTER 11}

Appearance of enzymes in plasma or urine following renal injury

\section{CHAPTER 12}

Estimation of renal ischemic damage from quantitated enzym leakage in plasma

\section{CHAPTER 13}

Loss of enzymes from viable kidney tissue 


\section{CHAPTER 14}

II1 and interferon gamma mediated cytotoxicity of kidney tubular cells

CHAPTER 15

Potentiation of ischemic acute renal failure by LPS is mediated by TNF

References

Summary/ samenvatting

Dankwoord

Curriculum vitae 


\section{PREFACE}

In our days the border between life and death is evidently put forward by the replacement of non-functioning organs for transplanted organs. It is therefore rather surprising that it is still impossible to tell from a donor organ whether it will be alive or dead upon transplantation. During the sixties preservation of organs became a natural part of human kidney transplantation, and a small but steady number of studies was published on the viability of donor kidneys dealing with a variety of parameters as diverse as the collour of the kidney, ultra-microscopical alterations and urine production. Most studies contributed to a slowly growing understanding of the pathophysiology of ischemic or preservation injury but did not report on successful viability tests. Until now no chemical or physiological tests are in common clinical use that can compete with the intuition of the experienced clinician.

Due to the moderate success of viability tests in experimental studies the interest in a simple and clinically feasible method to predict organ viability got lost "Gradually the opinion emerged that the concept of organ viability was actually an oversimplification of the processes leading to death. These processes appeared to have a complicated time course, affecting different levels of structural and cellular organization of the organ at different time intervals. The existence of a "point of no return", discriminating reversible from irreversible organ damage which would provide a sound basis for viability assessment, was seriously doubted.

There was still another reason for a decrease of interest in viability tests. In the pre-cyclosporin era rejection of the graft was certainly the major culprit of the limited success of clinical transplantation. The solution to this problem was considered the most effective way to increase transplant success rate and became the prime goal of research efforts. As after the introduction of cyclosporin and a number of sophisticated supportive therapies, organ rejection is satisfactorily under control, new angles become apparent which receive new priority.

At present the shortage of donor organs unmistakably hampers the current putative possibility to help all patients in need for a transplant organ. Future prospects are even worse. It has been calculated that today the need for kidneys doubles the supply. Unfor tunately, the disparity between supply and demand will probably increase as the proportion of elderly people within our population will grow in the next decades. This change in the age structure negatively influences the balance of potential donors and potential recipients. A number of possible solutions have been intensively discussed in the last few years without leading to a way out. The development of an artificial kidney is unlikely to offer a definitive solution as it might only replace the kidney as an "endogenous hemodialyzer" unable to perform hormonal renal functions. The use of kidneys from non-related living donors is not acceptable from an ethical point of view. Similarly, organs from primates are not accepted apart from immunological problems that have to be solved first. Therefore, a more efficient use of the potential source of organ donors seems at present to be the only realistic way to cope with organ shortage.

An important way to improve the current use of potential donors is to relax the criteria for donor selection. A new policy towards the acceptance of the so-called non-heartbeating donors may in this way be regarded to result in an appreciable enlargement of 
the donor pool. These donors have in common that they die in a rather uncontrolled situation from circulatory arrest. Harvesting of the kidneys is preceded by rapid in situ cooling of the organ through a catheter that is inserted via the femoral artery into the abdominal aorta. Once cooling of the kidneys has been achieved nephrectomy can be performed. The quality of these kidneys is thus likely to be more diverse because a number of kidneys may have suffered ischemic injury from circulatory instability before donor nephrectomy. Preliminary reports on the success rate of non-heartbeating donor kidneys are encouraging. Therefore the major drawback for general use of non-heartbeating donor kidneys is not the risk of some ischemic injury to the donor kidney but rather the uncertainty about the extent of possible ischemic damage. Transplantation of nonviable kidneys is not acceptable from an ethical point of view. In addition, it may offer a serious threat to the success of a retransplantation. Renewed interest in a method to discriminate viable from non-viable kidneys is therefore obvious. The direct and urgent clinical importance of viability assessment is likely to give new impetus to experimental studies on this subject in the near future.

The present thesis comprises a number of experimental studies that have been performed to explore possibilities in monitoring viability of donor kidneys. Most experiments were performed in dog, some in rat. Although not the principal aim of this study, a number of pilot experiments were started in the clinical setting, some of which are mentioned in this thesis.

It is fascinating to recall the experience of the person who first recognized that the death of the body does not necessarily coincide with the death of the individual organs themselves. It might have been a soldier in some ancient war looking at a beating heart in the crushed chest of a beheaded body. Such a cruel observation actually presents the phenomenological basis for current cadaver transplantation. The observed disparity in time in the process of dying, also illustrates the difficulties one may encounter in defining death. The example of the paradoxically beating heart in the beheaded body may be scaled down to the level of intracellular processes. Some cellular mechanisms can function apparently normal whereas others suggest inevitable death. The transition from viable to non-viable is obviously a time consuming process with a time schedule that may differ according to the level of observation. It is therefore unlikely that the assessment of the viability of an organ can be achieved by monitoring one single event at one single level. In the present study three different processes each at a different level were examined on their role in the development of renal damage due to ischemia. First, the cellular energy metabolism was studied representing an intracellular mechanism closely related to general metabolic processes. Second, the leakage of macro-molecules, i.c. enzymes, from damaged cells was examined as a symptom of lost homeostasis between the intra and extracellular environment. Finally, the involvement of the inflammatory response to tissue damage was studied as an extrarenal factor which may influence the eventual extent of ischemic damage.

(1)Attention has been paid to the role of the energy metabolism in processes leading to ischemic cellular damage and organ death. For many years the obvious crucial role of the adequate supply of high energy nucleotides for the cell to maintain viability has been suggested. The actual importance, especially with respect to the assessment of viability 
by measuring the tissue content of these metabolites has not yet been established. An answer to this problem was expected from recently developed, sophisticated techniques. High performance liquid chromatography technology allows to measure the tissue content of high energy nucleotides and their degradation products which are produced in a catabolic state during ischemia. With this technology it is possible to study changes at the level of the energy metabolism induced by ischemia as well as other factors during organ preservation and transplantation. If these changes appear to be characteristic they might be of great importance to the development of a viability test.

(2)The second part of the study is dedicated to the loss of cell integrity, due to ischemia, at a structural level. A progressive increase of membrane permeability has been suggested as a pivotal sign of cells destined to die. Thus the appearance of cellular constituents in the fluids surrounding the cell should evidence the presence of dying cells. Proper quantitation of the leaked cell content should allow to determine the extent of tissue damage. Enzymes were chosen as representative elements of the intracellular content as the biochemical assessment of a number of these proteins is routinely performed and their kinetic behaviour in the extracellular compartments has been well documented. In studies on the assessment of myocardial infarct size a mathematical model had been developed to estimate the quantitative amount of enzyme release from cells into plasma. In studies on renal ischemia, however, such attempts have not been previously described. Hence a number of assumptions underlying the model of quantitation of circulating proteins had to be verified in the specific situation of the kidney. If successful, this approach allows first to estimate the extent of tissue damage by serial plasma sampling during reperfusion of the ischemically damaged organ. Second, information on the tissue loss of enzyme might reveal a correlation between the duration of the ischemic interval and the corresponding extent of cell death.

(3)Increased attention is currently focused on the role of inflammatory mechanisms on the eventual outcome of organ injury following a period of ischemia. It has been recognized that mechanisms associated with reperfusion may influence the impact of the original ischemic insult both in a positive and negative sense. With respect to the latter, the role of invading neutrophils has attracted attention. Previously, the damaging effect of accumulated neutrophils was considered to result from microvascular obstruction. Currently, a number of direct interactions between neutrophils and ischemically damaged parenchymal cells are thought to play an important role in addition to the mechanical concept of obstruction. In the present study a direct toxic effect of substances released from inflammatory cells on renal parenchymal cells has been examined. Furthermore, an interaction between ischemic tissue injury and a general inflammatory response has been recognized suggesting a reciprocal stimulation.

These three approaches are introduced and discussed in section I of this thesis, each in one chapter, against the background of important findings in the literature. In section $\mathrm{II}$, individual experimental studies are reported, including materials and methods, similarly as they have been published or have been submitted for publication in scientific journals. 


\section{PART I}

\section{CHAPTER 1}

\section{INTEGRITY OF THE CELLULAR ENERGY METABOLISM DURING ISCHEMIA, HYPOTHERMIA, AND REPERFUSION}

\section{INTRODUCTION}

A quarter of a century ago a group of German investigators published a detailed and extensive quantitative description of the nucleotide behaviour in kidneys (Gerlach,1963). Catabolic pathways during ischemia as well as the postischemic recovery of the nucleotide content were studied to find an explanation for the remarkable ischemia tolerance of renal tissue in comparison to heart and brain tissue. A possible relationship between renall cellular energy metabolism and renal viability was suggested. Shortly thereafter, the first human renal transplant was performed. This new development stimulated the search for methods to store donor organs between harvesting and transplantation. Within the scope of this new field of interest soon the need arose to have a method to assess the viability of the donor kidney during or following storage other than by transplantation itself. The potential usefulness of monitoring tissue energy metabolism to assess viability was recognized leading to a large number of articles on this subject hitherto. In those days a valid viability test would have allowed to perform large series of experiments to evaluate new preservation techniques without the need for difficult and time consuming transplantations in animal experiments. Besides, the study of viability served a general interest in the nature of ischemic injury to find a more rational approach to organ preservation (Calman,1974). The application of a viability test in the clinical setting was considered useful but did not have the compelling need as it has today.

Current organ shortage and even worse prospects for the future forces the relaxation of donor selection criteria. Kidneys with ischemic injury from donors with circulatory instability may become acceptable for transplantation even though they may gain only life supporting function at some delay after transplantation (Kootstra 1984). As the transplantation of kidneys that will never function, however, is still a undesirable complication, a method to predict the viability of an organ upon transplantation has obviously become an indispensable tool.

From a historical point of view the use of the cellular nucleotide homeostasis in viability assessment may seem fully explored without success with respect to clinical application. During the early eighties the opinion grew in the field of organ preservation that the initial approach to the relation between energy metabolism and viability was rather too straight to be fruitful. A new impetus for a more fundamental approach came from distantly related fields of nephrology and physiology. Some recent experimental findings as well as new technical developments seem to justify now a pragmatic application of adenine nucleotide measurements in the clinical setting.

\section{VTABILITY}

The fact that there is still no simple and reliable method to distinguish viable from nonviable organs is obvious if one takes into account that the mechanisms of cell death are 
incompletely understood. Detailed descriptions of the chain of events leading to cell death are available but the key event, responsible for the irreversible nature of the process has not been defined (Duvall,1986). Several techniques to demonstrate the end stage of cell death are available. In case of viability assessment, however, the aim is to predict death from events during the course of dying rather than to determine the death of cells at the end of the process.

\section{Energy metabolism}

What are the reasons to believe that the cellular energy metabolism plays a crucial role in the process of dying? The metabolism of living organisms is based on the balance of energy yielding and energy consuming reactions. The tight coupling of these reactions is largely mediated by the ontogenitically conservative adenylate system. Its metabolites, AMP, ADP, and ATP may represent the ability of the cell to receive energy, to store it to some extent, and to provide the energy to metabolic reactions. Thus, the maintenance of the adenylate homeostasis is of utmost importance for cells to maintain viability. Since ischemia is known to induce rapid and striking changes of the tissue content of energy metabolites the persistent interest in the role of the energy metabolism on the loss of viability is obvious (Hochachka,1986).

\section{Value of monitoring the energy metabolism}

To provide full justification for the particular significance of the energy metabolism to viability some assumptions are still to be verified. It has often been claimed that disturbances of the energy metabolism play a pivotal role in the initiation of fatal injury (Bore,1979). It is, however, possible that the energy mediating system may be the most ischemia resistant mechanism of the cell. In other words, cells may have an intact energy metabolism and simultaneously develop structural or metabolic injury which appears to be fatal only after some time. The way in which the cellular energy metabolism is involved in cell death can therefore be described in two opposite concepts. First, a reduction of the cellular tolerance to a temporarily low capacity of the energy metabolism and secondly, the occurrence of a single lethal event in ischemia-induced changes of the energy metabolism that will irreversibly lead to cell death. These concepts are important to the nature of the relationship, if any, between the energy metabolism and viability as they indicate whether it is a causal relationship ar a time course relationship.

A second more practical assumption is that we are able to measure changes in the homeostasis of the energy metabolism by simple determination of the tissue content of energy metabolites. In experimental studies it is generally assumed that the latter is actually possible whereas the discussion is exclusively focused on the effect of changes. However, one has to bear in mind that our current technical tools may be inappropriate.

There is still a third assumption which needs attention. The assessment of viability of tissues is generally the aim of our biochemical assays whereas the prediction of the viability of an organ is the real aim (Fahy,1982). Among others, two reasons may be put forward that urge caution in this respect. Areas of cell destruction due to ischemia may be heterogeneously distributed dependent on the ischemia sensitivity of individual cell types (Brezis,1985). The architectural integrity may be disturbed with subsequent functional disorders whereas the majority of cells appear viable (Fahy,1982). Hence, assessment of cell viability may falsely indicate a viable organ. Furthermore, the development of spe- 
cific metabolic disturbances during ischemia may only become injurious if following the ischemic interval recirculation of blood is started and the metabolic rate of the cells is forced to rise. So the outcome of a viability test may be largely dependent to the moment at which it is performed.

\section{Definitions}

An obvious difficulty arising from these arguments is the lack of consensus in using the term viability. It may thus be impossible to compare the outcome of a variety of experimental studies with different definitions of the term viability. Assuming viability as a description of the state of events which possibly leads to death is not adequate as appears from the discrepancy between viability of individual renal cells and the viability of the organ as a whole. The viability of the organ as a whole, should actually be a functional description as it is coupled to the ability of the organ to sustain the life of the organism to which it belongs. In experimental studies, life sustaining function is often accepted as the ultimate proof of viability. The most important factor which determines this concept of viability secondary to the effect of the original ischemic impetus is the time the kidney is allowed to recover. The length of this time is mainly determined by the sensitivity of the organism to inadequate function of the organ. With complete lack of function the recovery time for kidneys, livers, and hearts is in the order of 3 days, 24 hours and a couple of minutes, respectively. Defining viability in this way leads to a number of various interpretations dependent on the organ which is concerned. This definition is therefore still no final judgement. The recovery time is not really fixed in both experimental studies and in clinical practice as lack of renal function may be temporarily supported by hemodialysis. These considerations indicate that the term viability should be clearly defined in each case in which it is used.

In studies on the role of the energy metabolism in ischemic injury, difficulties with the use of the term viability are in full length present (Pegg,1986). If the importance of the cellular energy metabolism to the loss of cellular integrity is discussed, viability is used with respect to the transition from reversible to irreversible damage, reflecting a chain of events occurring at the (sub)cellular level. If, however, viability is discussed in relation to viability assessment of organs for transplantation, it reflects the likelihood at which the injured organ will be able to recover functionally to a certain, satisfactory extent after some time following transplantation.

\section{PURINE NUCLEOTIDES}

The cellular pool of purine compounds consists of a wide range of metabolites with various functions. These metabolites all contain a purine base including adenine, guanine, hypoxanthine or xanthine. Cyclic AMP and GMP act as second messengers in hormonal information transmission whereas a number of adenine nicotinamides and adenine flavines are known as important co-enzymes. In the context of the present discussion the adenine tri-, di-, and mono-phosphates are considered. These compounds are generally involved in energy mediating processes in the cell. In addition, they act together with the guanine nucleotides as intermediates in a variety of metabolic processes. Moreover, in the tri-phosphate and deoxytri-phosphate form they are important precursors in RNA and DNA synthesis (Atkinson,1968;Buhl,1978). The role of the purine metabolism to the normally functioning cell is oversimplified by considering only the energy mediating 
aspects. Since the energy metabolism is, however, supposed to play a major role in the development of tissue damage during ischemia and hypothermia, these aspects will receive most attention.

\section{Turnover}

The turnover of purine compounds in the body is balanced by two generative pathways on one hand and the excretion of uric acid, as the final break down product, in urine and to a lesser extent in faeces, on the other hand. Conversion of phosphoribosyl pyrophosphate through various intermediate steps to IMP which in turn is convertible to AMP represents the de novo synthesis pathway of adenine nucleotides. A second way of nucleotide generation is offered by the reconversion of hypoxanthine, guanine and adenine as produced by nucleotide break down or as provided by dietary food intake (Mur-

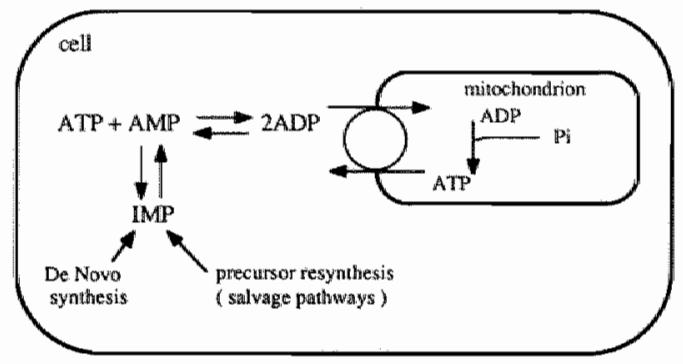

Figure 1.1.: Simplified scheme of adenine nucleotlde turnover.

ray, 1971). The ATP production is largely located in the mitochondria by the process of oxidative phosphorylation. Energy from mitochondrial respiration using $\mathrm{O}_{2}$ and substrates from the Krebs cycle is used to produce ATP through the binding of ADP and inorganic phosphate. Oxidation of, among others, fatty acids and glucose delivers the energy for ATP synthesis. To a small extent ATP is produced in the cytosol by substrate level phosphorylation. This mode of energy production plays a role in ischemic conditions in which lack of oxygen hampers mitochondrial respiration (Hochachka,1986). In some studies the respiratory control index (RCI) has been used to describe the energy state of the cells (Southard,1984). This index is a reflection of the ballance between energy consumption and production under various conditions. The availability of ADP and inorganic phosphate to the mitochondria is dependent on ATP hydrolysis by ATP consuming processes in the cell. Thus, the respiratory control index illustrates how the energy production keeps pace with the energy consumption. This close coupling is the key feature of the intact cellular energy metabolism. The coupling is mainly maintained by the action of the adenine nucleotide translocase activity across the mitochondrial membrane. The preservation of the integrity of the energy coupling mechanism has been suggested to be crucial in the preservation of tissues for transplantation, outside the body in oxygen-free conditions (Feinberg,1982). 


\section{RENAL ADENINE NUCLEOTIDE CONTENT}

\section{Introductory remarks}

Direct measurement of purine compounds in renall tissue reveals the actual concentration at a certain moment as well as the balance of the individual compounds. Multiple measurements in time give important additional information as they can tell whether the nucleotide homeostasis is either in balance or in a catabolic or anabolic state. It should be stressed, however, that the determination of the nucleotide content in itself does not give any information on the turnover rate of nucleotides. If their content drops the consumption is obviously higher than the synthesis process. Whether the consumption is actually increasing or decreasing remains unknown. To provide a picture of the energy state of the cell results of measurements are therefore usually interpreted in view of theoretical considerations, previous experimental experience and by taking into account the conditions in which the measurements were performed.

\section{Methods}

A number of methods have been developed to perform the determination of purine compounds. In most studies ATP, ADP, and AMP are measured using enzymatic assay techniques. In earlier years a paperchromatography method was employed (Gerlach,1963). This time-consuming technique lost its popularity but it offered the opportunity to determine a large number of other purine compounds as well as pyrimidine compounds in addition to the adenine nucleotides. Later ionexchange chromatography was used with similar possibilities to measure spectra of purine compounds, however, without di- and tri-phosphates (Busch,1968). With enzymatic assay techniques only few attempts have been undertaken to determine adenine nucleotide degradation products as well (Buhl,1978). The most convenient way to assess purine compounds is most likely the application of sophisticated HPLC technology (Wynants,1985). Recently, this technique has been adapted to determine an almost complete spectrum of adenine and guanine nucleotides as well as their degradation products (chapter 8). A completely different technical approach is presented by ${ }^{31} \mathrm{P}$ nuclear magnetic resonance (NMR). Changes of the ATP content can be non-invasively and continuously monitored with this method (Marschall,1985;Ross,1986). Unfortunately, no degradation products can be determined whereas the detection of ADP and AMP does not allow precise quantification. An advantage of this method when applied in studies on ischemic tissues is the possibility of simultaneous determination of $\mathrm{pH}$ changes.

\section{Substrate for measurements}

For all these analytical methods, except the ${ }^{31}$ P NMR technique, tissue specimen are needed. Since the individual nucleotide levels rapidly respond to arrest of the oxygen supply, particularly in renal tissue, harvesting of tissue samples may induce in itself ischemic changes in the biopted tissue. To stop energy consuming processes as soon as possible tissue samples are generally frozen in liquid nitrogen immediately. In small experimental animals whole kidneys can be cooled by a freeze clamp technique. In larger animals various sample techniques have been developed. The efficacy of such a technique is of great influence to the assessment of the level of individual nucleotide compounds in fresh kidneys with high di- and tri-phosphate levels. Thus, comparison of, especially, control values among various studies is difficult. It has been demonstrated, 
however that within the time needed to harvest a tissue sample and obtain metabolic arrest the sum of the adenine nucleotide content is not affected (chapter 4).

A few attempts have been reported in which the tissue nucleotide content is estimated indirectly by determination of degradation products in the effluent of the kidney assuming that these degradation products are washed out during reperfusion following ischemia (Buhl,1976;Takenaka,1980). Application of this technique is limited to storage procedures. If this method is valid, however, a convincing advantage is that there is no need for harvesting tissue specimen.

Measurements of renal purine compounds in the clinical setting have only recently be employed. Before, the risk for complications by the harvesting of tissue samples was considered non acceptable (Buhl,1978). It has been shown now that biopsies as small as 6 $\mathrm{mg}$ wet weight can be safely obtained from human kidneys during storage as well as following reperfusion (chapter 4 and 8 ).

\section{Tissue content}

It should be noted that the assessment of levels of purine compounds represents average values for a number of different cell types. With ${ }^{31} \mathrm{P}$ NMR technique it is possible to detect signals in cortex and medulla separately (Freeman,1986). In most studies, however, the content of purine compounds in cortical tissue only, is considered. As over $80 \%$ of the cortical tissue on weight base consists of proximal and distal tubular epithelium these cell types are most likely responsible for the measured changes in nucleotide content. In as much this simplification is a source of error to the interpretation of the measurements is unknown. The nucleotide homeostasis in the medulla is regulated differently (Urbaitis,1984). In addition, the adenine nucleotide content is similar but less reactive to cessation of the oxygen supply.

\section{ATP}

Although the adenylate system (interconversion of AMP and ATP in ADP) is universally involved in the energy metabolism of cells, the cellular concentration of these compounds varies among species and tissue types. Due to the characteristic extremely rapid fall of ATP in renal tissue upon circulatory arrest comparison of ATP values among different studies reveals more about the efficacy of the tissue specimen freezing techniques than about the actual ATP tissue levels in physiological conditions as explained before. The real ATP concentration can be roughly estimated by extrapolation from the sum of the nucleotides if one takes into account that the total adenine nucleotide pool is not affected by short periods of ischemia (approximately less than 30 seconds) and the energy charge which reflects the internal balance among the individual nucleotides: (ATP + $0.5 \mathrm{ADP}) /(\mathrm{ATP}+\mathrm{ADP}+\mathrm{AMP})$, ranges from 0.7 to 0.9 in normally functioning tissues (Atkinson,1973). ATP values of about $10 \mu \mathrm{mol} / \mathrm{g} \mathrm{dw}$ at a energy charge of 0.88 have been measured in canine kidneys using HPLC techniques (Southard,1985; chapter 4). Quantitation of ${ }^{31}$ P NMR ATP signals delivers values in the same order (Freeman,1986). Such values are probably in close approximation to the actual physiological value. The ATP content is different along various nephron segments. In rat the lowest ATP levels are found in the medullar region, in the thin limb of the loop of Henle and in the papillary area. In the distal tubules the content ( $17 \mu \mathrm{mol} / \mathrm{g} \mathrm{dw}$ ) is more than twofold higher whereas in the thick ascending limb and in the proximal tubules the content is 13 and 10 
$\mu \mathrm{mol} / \mathrm{g} \mathrm{dw}$, respectively (Cunarro,1985). These data suggest that measurements in cortical tissue samples or in whole kidney samples represent average values of nucleotides which are mainly determined by the content in the distal tubules.

\section{Adenine nucleotides}

The total adenine nucelotide content in dog kidneys is $10-12 \mu \mathrm{mol} / \mathrm{g} \mathrm{dw}$ as has been reported by different groups using HPLC techniques (Sinsteden,1986; Southard,1986; chapter 4). In a large study using enzymatic assays a value of $3 \mu \mathrm{mol} / \mathrm{g} \mathrm{ww}$ has been found (Cunarro,1982). If the percentage dry weight is considered to be about $20 \%$ this value represents $15 \mu \mathrm{mol}$ on dry weight base. Others reported values in closer agreement to the HPLC results (Pegg,1984; Fischer,1980). In rat kidney the total adenine nucleotide content is rather similar to values obtained in dog. In rabbit, however, higher levels have been found, ranging from 15 to $20 \mu \mathrm{mol} / \mathrm{g} \mathrm{dw}$ (Buhl,1979).

Only a few studies are available on the assessment of purine compounds in human kidneys. The total adenine nucleotide content in cortical tissue is $10-11 \mu \mathrm{mol} / \mathrm{g}$ dw with HPLC techniques. A mean energy charge of 0.68 has been obtained in this study using cortical wedge biopsies (chapter 8). In whole kidney samples with enzymatic assay techniques a similar nucleotide content was found at a energy charge of 0.59 (Voss, 1970).

\section{Degradation products}

The level of degradation products is extremely low in fresh, nonischemic renal tissue. Nucleoside levels, adenosine and inosine, are less than $0.1 \mu \mathrm{mol} / \mathrm{g} \mathrm{dw}$ whereas the purine bases hypoxanthine and xanthine are not detectable using HPLC methodology. Normal tissue IMP levels range approximately between 0.2 and $0.5 \mu \mathrm{mol} / \mathrm{g} \mathrm{dw}$ (chapter 4).

\section{NORMOTHERMIC ISCHEMIA}

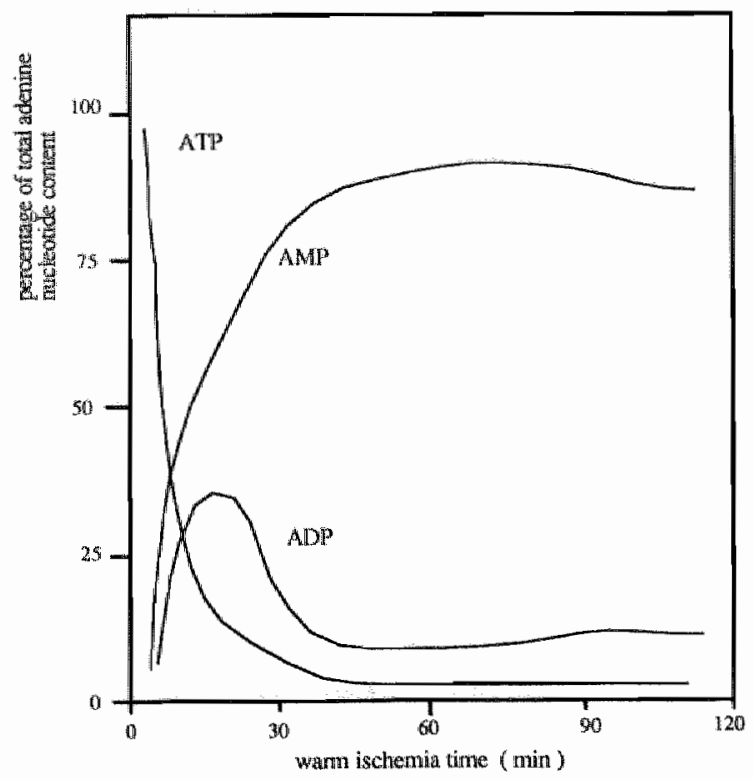

Figure 1.2.: Levels of the individual adenine nucleotides as a percentage of the total adenine nucleotide pool in canime kidneys during normothermic ischemia. Values are obtained from chapter 4. It is shown in this diagram that the ATP pool is rapidly depleted concomitant to a transient increase of ADP and a permanent accumulation of AMP. After $\mathbf{3 0}$ minutes of ischemia a balance among the individual compounds is achieved which does not change during the subsequent period of ischemia. 
Following cessation of the oxygen supply to the kidney due to circulatory arrest, the oxidative phosphorylation in the mitochondria ceases. As the production of ATP is impaired and the need for energy continues, it is theoretically amenable that the residual

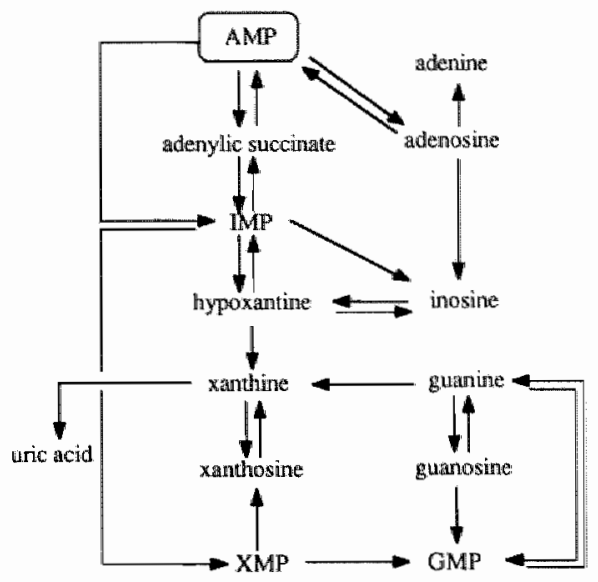

Figure 1.3.: Possible breakdown pathways of AMP

amount of chemically bound energy will be ecomomically redistributed to processes which are essential for the cell to maintain its integrity. The normal functioning tubular cell consumes about $9 \mu \mathrm{mol}$ ATP per minute per gram of tissue. If the same consumption rate is continued during ischemia the total ATP pool will be depleted within 1 minute (Freeman,1986;Mandel,1986). Most of the energy production is normally spend on the exchange of ions from and to the glomerular filtrate. These processes rapidly cease upon the oxygen deprivation. The regulation of the close coupling of energy expenditure, cell function and the maintenance of a minimum cellular metabolism is not precisely known (Hochachka,1986).

The equilibrium between the individual adenine nucleotides as represented in the adenylate kinase reaction ATP + AMP $=2 \mathrm{ADP}$ is supposed to play an important role in adjusting the balance of energy consumption and production as these substances may inhibit or stimulate reciprocally metabolic processes that influence their concentrations (Weidemann,1969). The effect of the response of the adenylate kinase reaction to ischemia is tissue dependent as important differences have been observed in brain, heart, liver, and kidney tissue (Busch,1968;Gerlach,1970;Schmidt-Mende,1966). The tissue specific response has been related to differences in ischemia tolerance but the underlying mechanism is not yet understood.

Catabolic pathways

After a certain time interval following the onset of the ischemic insult the adenylate pool is no longer kept constant and starts to decrease. Through the break down of AMP a spectrum of degradation products is generated which again shows some specificity to 
tissue and species (Busch,1968). If the ischemic insult persists the break down of AMP will theoretically proceed to xanthine or uric acid. The activity of the enzymes that are involved in each conversion however may show a different sensitivity to the ischemic conditions. As a results some degradation products may defimitively accumulate whereas others are transiently increased dependent to the length of ischemia time (Gerlach,1963). Some degradation products can potentially act as precursors in the regeneration of the adenine nucleotides (Murray,1971). The actual importance of these pathways in ischemia or postischemic conditions in the kidney is not yet known. A direct conversion of ischemia produced degradation products to AMP has not been demonstrated (Buhl,1979). Xanthine, uric acid and allantoin are end products which are nor-

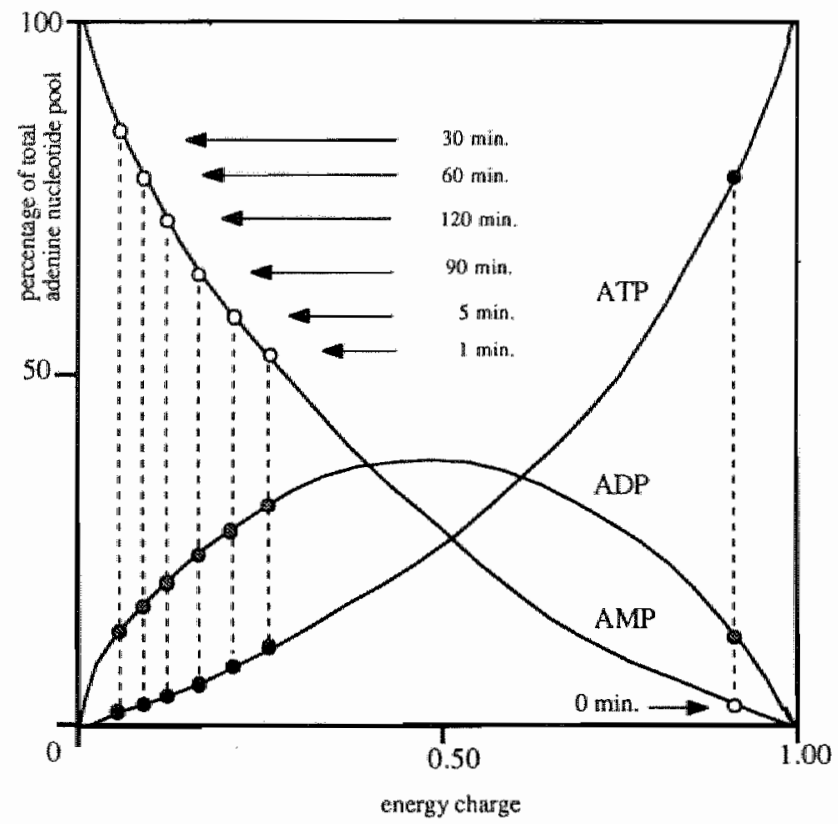

Figure 1.4.: This diagram shows the relative proportion of the individual adenine nucleotides, ATP, ADP, and $A M P$, as a function of the energy charge: (ATP + 1/2 ADP)/ (ATP + ADP + AMP) based on the adenylate kinase reaction $A T P+A M P=2 A D P$. The line drawings show the calculated relative concentrations of the individual adenine nucleotides when the adenylate kinase reaction is at equilibrium (Atkinson, 1968). The substituted data points represent actually measured concentrations of these metabolites at various intervals of warm ischemia (indicated in minutes at the bottom of the dotted lines) as a function of the energy charge (the dotted lines link values at the same time interval). The data suggest that despite prolonged ischemia time (120 minutes) the levels of the adenine nucleotide are regulated according to the adenylate kinase reaction at equilibrium. Thus, the postulated theoretical correlation between the individual metabolites in physiological conditions is maintained during severe ischemic injury. The measured concentrations of the adenine nucleotides were obtained from chapter 5 . 
mally excreted by the intestine or the urinary system (Quebbemann,1975). There is evidence that all degradation products except IMP are able to leave the cell (Buhl,1979). In postischemic tissue perfusion these metabolites may be in part or completely recovered in the effluent. There may be a role for circulating degradation products in regenerative pathways.

Two possible pathways for the break down of AMP have been described (Buhl,1979; Busch, 1968). If deamination is the first step, IMP will be produced which may be in turn converted to inosine, hypoxanthine and xanthine. However, when dephosphorylation is the first step to occur, adenosine will be produced with subsequent conversion to inosine and hypoxanthine. Present techniques to measure the content of purine compounds provide a static picture that do not allow to discriminate which pathway actually is followed. However, the accumulation of a particular product demonstrates that previous converting steps took place and that the subsequent converting step is less fast or is absent in the given circumstances.

\section{Degradation products}

During normothermic ischemia only limited accumulation of inosine and adenosine has been demonstrated in renal tissue (Osswald,1978; chapter 4). The concentration of these products always remains less than $1 \mu \mathrm{mol} / \mathrm{g} \mathrm{dw}$ up to 180 minutes of ischemia. It is in teresting to note that in heart tissue a profound increase of these products is found within the same time interval (Busch,1968). The degradation product adenosine has received much attention as strong vasoactive properties of this compound are known. In kidney

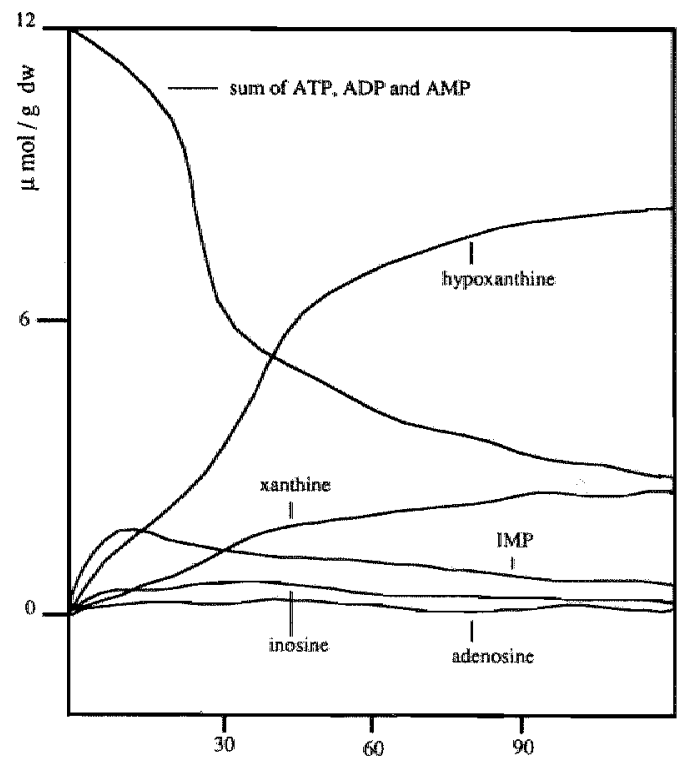

warm ischemia time (min)
Figure 1.5.: Changes in the levels of possible degradation products of the adenine nucleotides in cortical tissue of canine kidneys during ischemia (Data obtained as described in chapter 4). Abbreviations are: $\mathrm{HX}_{\text {, hypo- }}$ xanthine " $X_{\text {, }}$ xanthine; INO, inosine" $\mathrm{ADO}$, adenosine; $\mathrm{AN}_{\text {"sum }}$ of adenine nucleotides. The dotted line indicates the decrease of the adenine nucleotide pool along with ischemia time. Note the transient increase of IMP early during ischemia. A similar but much smaller increase within the first few minutes is observed in the adenosine levels. Hypoxanthine and xanthine are in quantitative respect the most important degradation products. Other degradation products, adenylic succinate, guanosine, xanthosine, and XMP only slightly changed in the same range of order as adenosine (not shown). 
minute amounts can induce a remarkable vasoconstrictive response of the intrarenal vessels. The importance of this phenomenon has been suggested in studies in which adenosine concentrations were measured in the nanomolar range. A rapid increase following ischemia was observed which peaked at 10 minutes and then gradually decreased. The characteristic absence of postischemic hyperemia in kidneys has been related to the effect of the accumulated adenosine in this organ (LeHir,1988;Osswald,1978). Quantitatively, however, hypoxanthine is by far the most important degradation product in ischemic kidneys. The characteristic accumulation of hypoxanthine was already recognized in 1945 (Kerr,1945). In dog, rabbit, and man the hypoxanthine level steadily increases along with the length of ischemia time (Buhl,1978; Voss,1970; chapter 4 and 6). In rat a somewhat different pattern is observed as the hypoxanthine accumulation is less whereas a concomitant and equal accumulation of xanthine is found (chapter 6). The conversion of hypoxanthine to xanthine is important as the enzyme mediating this step is assumed to be involved in the formation of radical oxygen metabolites during reperfusion (McCord,1985). These metabolites may be responsible for part of the damaging effect of ischemia-reperfusion associated injury. The presence of this enzyme, xanthine oxidase, however, has been demonstrated to be species dependent (Southard,1986; Granger,1986). Its rolle in the generation of oxygen radicals induced ischemic injury has therefore been doubted. In addition, it is unknown whether the increase in tissue xanthine levels results from hypoxanthine conversion or from other sources like guanine breakdown.

\section{Pool of cellular purine compounds}

Since no further break down beyond xanthine is observed during ischemia the adenine nucleotides and their degradation products might be considered as a pool with a fixed content. It should be noted, however, that the break down of other purine and pyrimidine compounds as a result of ischemic injury may contribute to the accumulation of similar degradation products (Gerlach,1963). The guanine nucleotide concentration is about $25 \%$ of the concentration of adenine mucleotides in fresh kidneys (rat, dog, and man) (Busch,1968; chapter 10). The depletion of this pool during ischemia is proportional to the depletion of the adenine nucleotides. Hence, part of the xanthine accumulation may originate from guanine nucleotide break down. However, it has been shown in rat kidneys that within two hours of ischemia, guanine is the major degradation product of the guanine nucleotides. The concentration of uridine compounds is less than $2 \mu \mathrm{mol} / \mathrm{g} \mathrm{dw}$ in normal kidneys with a negligible loss during ischemia. The renal cytidine content is even smaller $(0.1 \mu \mathrm{mol} / \mathrm{g} \mathrm{dw})$ and is also unlikely to influence the accumulation of degradation products. The contribution of RNA break down to tissue levels of degradation products is uncertain. In rat no increase of purine compounds following RNA break down has been observed. In rabbit, RNA hydrolysis has been assumed to induce an increase of $4-5 \mu \mathrm{mol} / \mathrm{g} \mathrm{dw}$ of the purine metabolite pool after 3 hours of ischemia (Gerlach,1963; Busch,1968). Therefore, this influence should be accounted for if changes in the total purine pool are discussed. Until now no consistent results have been reported on changes of the total pool of cellular purine compounds during normothermic ischemia. In rat a decrease of this pool of about $16 \%$ has been found. In contrast, in rabbit this pool increases during ischemia (Buhl,1978). In dog this pool tends to increase (chapter 4). 


\section{REPERFUSION FOLLOWING ISCHEMIA}

Dramatic changes may occur at a metabolic level of the cell in previously ischemically damaged tissue during recirculation with well oxygenated blood. On the one hand restoration of blood perfusion is the only way to save the cell from an inevitable death. On the other hand it can be regarded as a challenge to the disturbed metabolic homeostasis of the cell. The death of cells at borderline viability may be accelerated. Cells with a declining membrane integrity are suddenly encountered with osmotic forces. Inadequate processing of metabolic substrates may create toxic intermediates and induce further damage. The impact of such negative side-effects is difficult to establish but a growing number of observations makes the occurrence of what has been called 'reperfusion injury' likely.

Recovery of the energy metabolism

The energy metabolism is able to rapidly adapt to the renewed normoxic conditions. In canine kidneys after an ischemic interval of $\mathbf{3 0}$ minutes the energy charge is back at a pre-ischemic level within 5 minutes of reperfusion and the replenishment of the nucleotide pool has started. Within one hour of reperfusion tissue nucleotide levels have almost returned to pre-ischemic levels (chapter 9). Apparently, the cellular energy metabolism is capable to meet the energy requirements as well as to restore the high energy phosphate levels to normal after 30 minutes of ischemia. After prolongation of the ischemic interval to 90 minutes the rapid normalization of the energy charge is still present. The difference is that the increase of tissue content of nucleotides is much more slower than after $\mathbf{3 0}$ minutes of ischemia. These changes are generally accepted to be characteristic in renal tissue. However, in a number of studies some different observations were reported. In rats the tissue adenine nucleotide levels do not increase within 15 minutes of reperfusion following 25 minutes of ischemia (Zager,1986). Others found a rapid almost doubling of the nucleotide content in rat kidneys within one hour of reperfusion following 50 minutes warm ischemia (Arnold,1986). In one study in dog kidmeys a fall of adenine nucleotide levels within 5 minutes of reperfusion was observed. In the same study nucleotide levels were found to rise during warm ischemia rather than being depleted. These confusing observations suggest that methodological variations should be taken into account when comparing results from different studies.

\section{Recovery in relation to prior depletion}

Since the depletion of the adenine nucleotide store depends on the length of the ischemic interval the suggestion has been made that the postischemic nucleotide recovery is directly related to the nucleotide depletion during ischemia. This hypothesis is supported by findings in rabbit kidneys in which the rate of nucleotide resynthesis upon reperfusion is similar in kidneys with moderate and severe ischemic damage. Hence, the final level of nucleotides after some period of time upon reperfusion depends on the extent of depletion during ischemia before reperfusion is started (Buhl,1979). In a study in canine kidneys, however, it has been shown that the rate of nucleotide recovery is retarded after 90 minutes of warm ischemia in comparison to the recovery rate in kidneys after 30 minutes of ischemia. Here it is not solely the depletion of nucleotide levels during the ischemic interval that causes low postischemic nucleotide levels following reper- 
fusion but it is in addition an impaired capacity to regenerate nucleotides that causes a depressed recovery upon reperfusion (chapter 7). Studies on the regeneration of adenine nucleotides in incubated cortical tissue slices in vitro in the presence of precursors have shown that both the ischemic tissue as well as fresh tissue are equally well able to regenerate nucleotides as the fresh tissue. It was therefore suggested that the poor replenishment of the nucleotide pool after ischemic damage results from insufficient amounts of available substrates (Collins, 1979).

\section{Postischemic function}

In a number of studies the development of postischemic renal failure has been related to inadequate recovery of the energy metabolism. It might appear from these studies that the impairment of the nucleotide recovery which has been demonstrated early during reperfusion, persists during subsequent renal failure. Recent findings, however, have shown that even after 90 minutes of ischemia the depressed nucleotide regeneration early during reperfusion is more a delay in recovery rather than a definitive incapability to resynthesize nucleotides. Within 24 hours following reperfusion the tissue adenine nucleotide content has returned to normal pre-ischemic levels ( chapter 9 ). No recovery to life sustaining function of the organ as a whole is observed at that time. Additional evidence that the nucleotides levels per se do not reflect the functional potency of the tissue has been obtained in studies on the effect of exogenous supply of metabolic compounds. It is well established now that the level of tissue adenine nucleotide can be increased with the use of pharmacological agents. Especially, products including nucleotides or purine bases are effective. In addition, treatment with these agents has been successful in improving the quality of some functional renal parameters during postischemic reperfusion. However, important questions to the nature of these effects which are less obvious as they may seem to be, are still to be solved. Improvement of function, for instance, has been achieved without notable changes in the levels of nucleotides. Similarly, increased levels of nucleotides have been obtained without any beneficial effect on renal function. Finally there is no proof that putative precursors, such as exogenous nucleotides and purine bases, are actually incorporated in the endogenous nucleotide pool (Gaudio,1986; Weinberg, 1986; Shapiro,1987; Weinberg, 1988). Other mechanisms of action of exogenously supplied substrates which may indirectly lead to the observed effects, have to be considered. For instance, a number of purine compounds, if present in the extracellular compartment, are known to exert strong vascular actions ( $\mathrm{LeHir}, 1988$; Osswald,1978).

\section{Degradation products}

Hitherto no evidence is at hand that the degradation products which accumulate during the ischemic episode are involved in the regeneration of nucleotides during reperfusion. Within 5 minutes of reperfusion the decrease of the level of degradation products is not related to the increase of nucleotides in the same interval. Both, in kidneys after 30 and 90 minutes of ischemia the levels of degradation products fall to the same extent whereas in the latter group of kidneys no nucleotide regeneration is found (chapter 7 and 9). Theoretically several pathways of nucleotide synthesis using degradation products are available to the cell at a lower energy cost than de novo resynthesis. It is possible that the degradation products which are known to be able to freely pass the cellular mem- 
brane do not accumulate in the same compartment as the salvage pathways occur. Isolated kidney perfusion studies suggest that the degradation products are rapidly and quantitatively washed out from the ischemic tissue during reperfusion. A role for circulating degradation products in nucleotide resynthesis has to be considered.

\section{HYPOTHERMIA}

In general, (non-hibernating) mammals die at body temperatures below $15^{0} \mathrm{C}$. Their organs however, can stand reasonably well long term exposure to cold without any loss of function after re-establishment of normothermic conditions (Fuhrman,1943). Many factors might influence the tolerance to hypothermia including tissue type, and storage temperature. The cooling rate was considered the most important one. Surface cooling with ice-coild saline in polyethylene bags packed around the kidney induces a fall in core temperature to about $17^{\circ} \mathrm{C}$ within 8 minutes dependent on the size of the kidney. Notably,

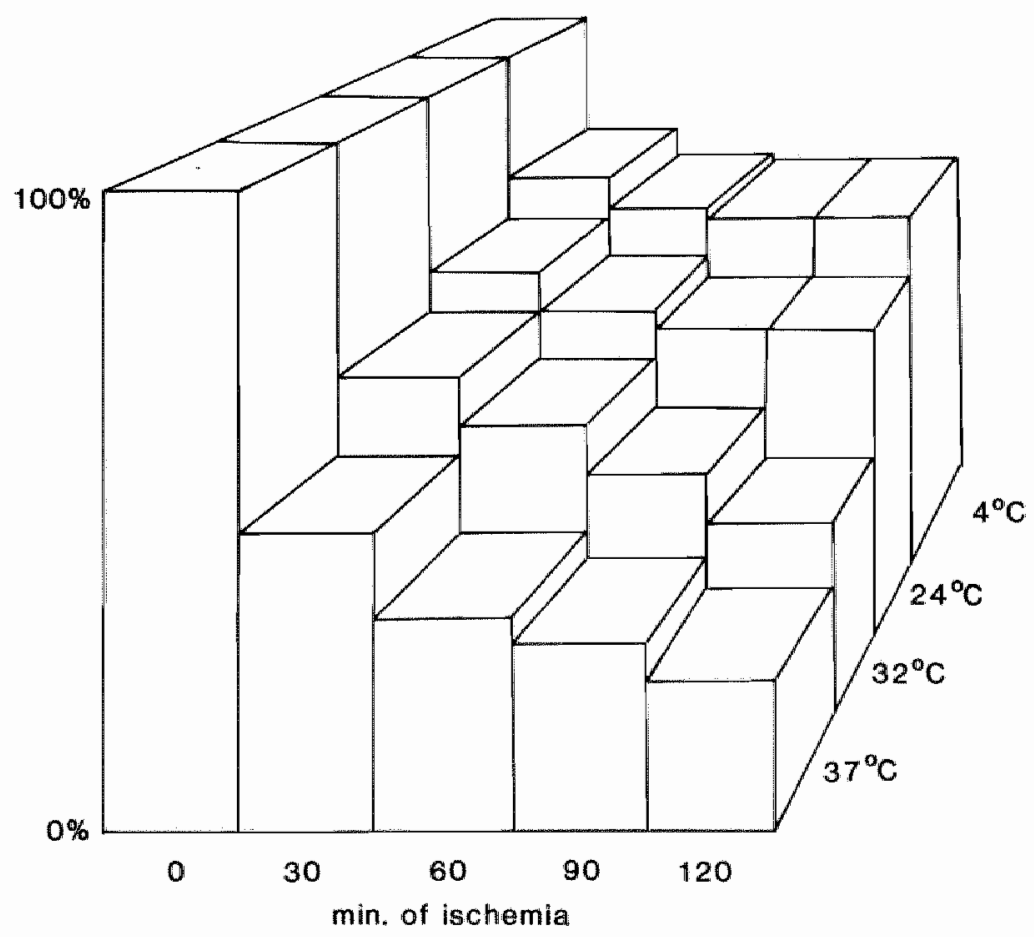

Figure 1.6.: This diagram shows the effect of temperature on the depletion of adenine nucleotides during ischemia. Canine kidneys were stored at $37^{0} \mathrm{C}, 32^{0} \mathrm{C}, 24^{\circ} \mathrm{C}$, and $4^{\circ} \mathrm{C}$ during 120 minutes following nephrectomy without flushing. At intervals of 30 minutes cortical biopsies were taken to assess the tissue adenine nuclleotide content. A clear reduction ws. control (100\%) of both the rate and the extent of nucleotide loss is obtained by even a small lowering of temperature during the ischemic interval. Data were obtained from 
large differences in temperature may exist in different areas of the kidney. Temperature differences of more than $12^{\circ} \mathrm{C}$ between the surface and the core of the kidney have been measured (Kerr, 1960). Later, successful preservation during 12 to 17 hours was obtained by cooling outside the body if kidneys were treated with heparin (Calne,1963). Further prolongation of the maximum preservation time of cold storage has only been obtained after the introduction of flushing to cool kidneys.

Role of the energy metabolism in hypothermia tolerance

The major questions concerning the role of the energy metabolism in the limitations to hypothermia tolerance can be defined as follows: first, is the endogenous level of energy metabolites and the production of energy sufficiently high to drive the sodium pumps effectively to maintain the cellular ion balance during storage, and second, is the energy metabolism capable to meet the higher demands when following reperfusion and reoxygenation the metabolic rate is increased and the physical properties of the cellular surroundings are rapidly changing.

The latter question will be addressed in the section on cold storage. With respect to the first question it should be noted that some cellular metabolic properties remain remarkably well preserved during hypothermia. The activity of the major enzymes involved in the process of gluconeogenesis is only moderately decreased during prolonged cold storage for as long as 11 days (Kesner,1977). Similarly, the coupling of oxidative phosphorylation in kidney mitochondria is still present at $5^{\circ} \mathrm{C}$ during 3 to 5 days as appeared from studies on isolated mitochondria (Southard,1980). In contrast, other metabolic processes are clearly affected by hypothermia. Adequate control of cell volume and ion balance is supposed to be a key feature of successful hypothermic storage of kidneys (Pegg, 1978). In membranes isolated from cortical cells a decrease of $\mathrm{Na}+\mathrm{K}+-\mathrm{ATPase}$ activity has been observed following hypothermic storage. The reduced electrolyte transport mechanism causes changes in the intracellular sodium content. This effect is dependent on the electrolyte concentrations in the storage solution (States,1972).

Processes in which the mitochondria are involved show various important changes in response to hypothermia. The activation energy for ADP stimulated respiration increases at low temperature as is apparent from a discontinuity in the Arrhenius plot (Southard,1983). At temperatures below $10^{\circ} \mathrm{C}$ the adenine nucleotide translocation activity is suppressed whereas the activity of the respiratory chain is largely unaffected (Southard,1980). The discontinuity in the Arrhenius plot has been related to alterations in the fluidity of membranes. Below a certain temperature a phase transition of the phospholipids in cellular membranes is observed, thus altering the activity of membrane bound processes. The reversion of this transition may be impaired after prolonged exposure to cold which may induce disruption of membrane integrity. These findings may explain in part the gradual decrease of the cellular adenine nucleotide content during hypothermia. Since the consumption of ATP continues, albeit at a lower rate, ADP will accumulate if the transport of ADP across the mitochondrial membrane is unable to keep pace with ADP production. Due to the adenylate kinase reaction this will lead to the production of AMP and to subsequent break down of AMP to degradation products. In this concept, the failure of cells to recover upon reperfusion is caused by a lack of ATP precursors. This state of energy shortage has been designated as the "purine deficit syndrome". However, it was recently recognized that the decrease of the nucleotide store 
does not continue along with storage time. The occurrence of nucleotide depletion appeared to be dependent on the amount of nucleotides that are still present in the cell rather than on the length of the hypothermic period. The exhaustion of the nucleotide pool leveled off at nadir levels. Interestingly, this nadir level did not correspond to loss of viability. In these conditions apparently, a new balanced state is obtained in which the AMP break down is most likely suppressed ( chapter 5). In this alternative concept, a purine deficit upon reperfusion is still possible but it also suggests that nucleotide depletion per se is probably not the major threat to cell viability during hypothermia.

\section{COOLING BY FLUSHING}

It has been empirically established that the maximum length of preservation time of kidneys by simple cold storage is considerably extended if kidneys are flushed during a short time interval with an electrolyte solution at $4^{\circ} \mathrm{C}$ before storage. The observations that pretreatment of kidneys with heparin and a brief period of perfusion of kidneys with heparinized autologous blood at $10^{\circ} \mathrm{C}$ (Calne,1963;Pegg,1964) markedly improved the effect of surface cooling, gave rise to the concept of flush cooling of kidneys for transplantation. Although flushing has become a standard technique in clinical transplantation the mechanisms underlying the effect have not been completely elucidated. This is illustrated by the fact that currently presented modifications of this technique, more than 15 years after the introduction of the generally used Eurocollins flush solution, offer remarkable better results.

\section{Sparing of nucleotides}

One of the explanations for the effect of flushing is related to adenine nucleotide metabolism. Since a more rapid cooling of kidneys is obtained by flushing with a solution at $4^{\circ} \mathrm{C}$ than by surface cooling at $4^{\circ} \mathrm{C}$, it was assumed that the rate of adenine nucleotide break down would be proportionally reduced (Pegg,1978). Thus, flushing might have a nucleotide sparing effect. Recently, however, it was found that the loss of nucleotides during 8 minutes of flushing with Eurocollins in freshly excised kidneys is significantly larger than with surface cooling (chapter 5 and 10). In kidneys with a depleted adenine nucleotide pool from a pre-existing ischemic insult these differences are not found. The nucleotide pool does not further decline during flushing time. These findings point out that the beneficial effect of flushing with Eurocollins is unlikely to be related to the effect on the energy metabolism. The increased nucleotide loss by flushing might in part result from the inhomogeneous distribution of the flush solution in the renal tissue due to vasospasm (Kallerhof,1986). In bad perfused areas the cooling is ineffective and the catabolism of nucleotides continues at a high rate. An alternative explanation is that the installment of a new osmotic equilibrium between the intra- and extra-cellular space by the flushing solution in actively pumping cells may affect the membrane permeability leading to a partial loss of nucleotides.

\section{COLD STORAGE}

In the previous paragraphs the general effect of low temperatures on the energy metabolism has been discussed as well as the effect of cooling by the flushing procedure. In this paragraph we will examine the effects of long term storage on the recovery of the energy metabolism when kidneys are reimplanted. 
A

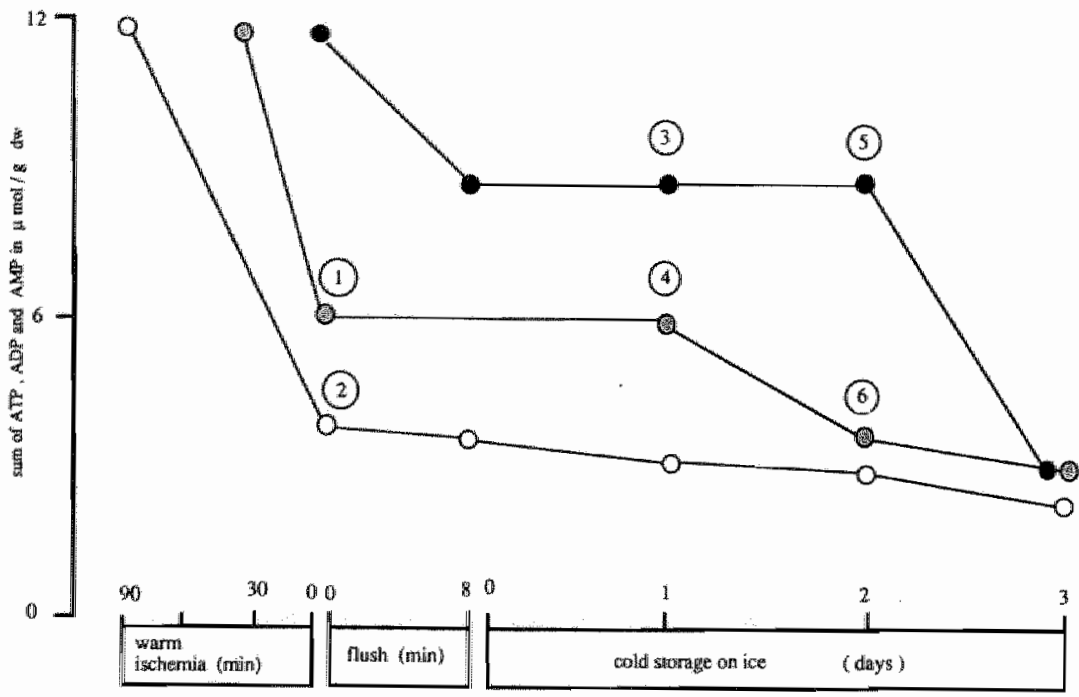

Pigure 1.7." Diagram sthowing tissue adenine nucleotide levels during simple cold storage with or without prior exposure to warm ischemia (closed cicrcle, no additional warm ischemia; shaded circle, 30 minutes of ischemia; open circle, 90 minutes of ischemia). Data were based on studies described in chapter 6 and 10 . Adenine nucleotide levels were determined in cortical tissue specimen from canine kidneys using HPLC methodology. The flush procedure was performed with Eurocollins during 8 minutes at $4^{\circ} \mathrm{C}$. In the small diagram (B) the increase of the sum of ATP and ADP, and the sum of ATP , ADP, and AMP is shown within 60 minutes of reperfusion either in situ $(1,2)$ or following autologous implantation ( 3 to 6$)$. Conditions prior to reperfusion are shown in the large diagram (A) at the corresponding figures. e.g. the bars on number 4 show the nucleotide recovery within 60 minutes of reperfusion in kidneys that were subjected to 30 minutes of warm ischemia, flushed with Eurocollins and stored for 24 hours on ice before reperfusion.

B

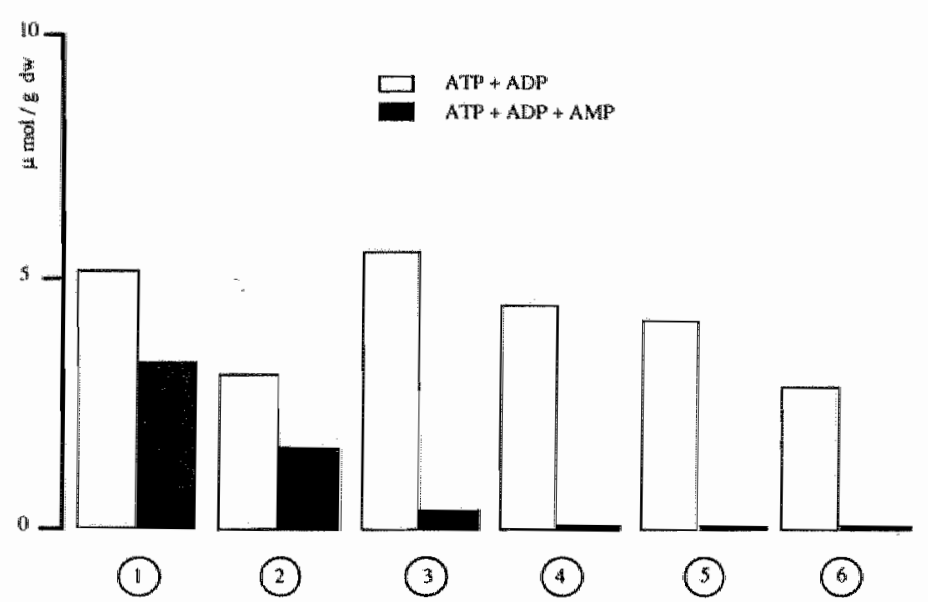




\section{Storage on ice}

Simple storage of kidneys on ice in an intracellular electrolyte solution with which the kidney has previously been flushed is the most commonly applied preservation method. With this technique a reduction of the nucleotide loss is achieved. During 24 hours storage a depletion to about $70 \%$ of control is observed. The depletion continues during the next day and then gradually levels off at one third of the original pool between 72 and 120 hours (Southard,1977; Fischer,1981). Recently, it has been demonstrated in dog that the adenine nucleotide loss in non-ischemically injured kidneys during the first 24 hours may be due to the effect of the flush procedure (chapter 5 and 10). A decrease of the total nucleotide pool during the storage period itself is only observed when storage time is extended to 48 hours (chapter 5).

However, in dog kidney, ATP is clearly almost completely depleted within the first day of storage and remains at a constant low level for at least two days more. In rat kidneys a different pattern is observed. The nucleotide pool is reduced to about one third of control within 24 hours using a hypertonic citrate solution. The ATP level remains surprisingly high, comprising 50\% of the total adenine nucleotide pool (Marshall,1978). Degradatiom products accumulate in a rather similar way during cold storage as during normothermic ischemia. Hypoxanthine and xanthine are quantitatively the most important degradation products. Accumulation of other degradation products is hardly noticeable. It has been demonstrated, however, that IMP levels rapidly increase and remain at a higher level than in fresh kidmeys and in kidneys subjected to normothermic ischemia for more than 30 minutes (chapter 5). The observed increase of IMP levels during hypothermia may result from the general retardation of the break down process of this nucleotide since early during normothermic ischemia a slight and transient increase of IMP is found. An alternative explanation is that the route of AMP break down during hypothermia is different (chapter 5). Comparison of the amount of degradation products that is formed and the amount of nucleotides that is lost during hypothermia, is hampered by the fact that the flushing procedure also induces a loss of metabolites. Part of the degradation products that are present in the renal tissue before flushing as well as the degradation products that are formed during the flushing procedure are most likely washed out (Takenaka,1980; chapter 5). It is therefore difficult to determine whether during cold storage degradation products are formed by processes other than the break down of nucleotides.

\section{Machine preservation}

Hypothermic continuous perfusion is the second most generally applied preservation technique. Comparison of the renal metabolism during hypothermic continuous perfusion among various studies is hampered by the use of a large number of different perfusion solutions. Hypothermic continuous perfusion is generally considered as a more biological alternative to the physical approach of simple cold storage in an electrolyte solution. When the perfusion solution is thought to replace the function of the blood in this biological concept, it is clear that a wide range of metabolites alone or in combination have to be examined to achieve the ideal composition of the perfusion solution. The direction in which the development of these solutions should proceed is not yet clear. The addition of some obviously important substrates like carbohydrates and free fatty 
acids to the standard solution with albumin did not change the quality of preservation (Fischer,1980). Glucose, glycogen, and lactate levels are low in kidneys during storage and remain at the same level during at least 48 hours. If glucose is supplied during preservation some break down products of glucose are found. This treatment, however, does not influence the level of the energy metabolites (Fischer,1979; Fischer,1980).

Changes of the levels of purine metabolites during machine perfusion are markedly different from changes during storage on ice. In rabbit kidneys the total adenine nucleotide content is maintained at control levels during the first 24 hours of preservation and drops during the next 24 hours to about two third of control which is more or less similar as has been reported for simple cold storage. The difference, however, is that the levels of the two high-energy nucleotides, ATP and ADP, remain high and no accumulation of AMP is observed (Pegg,1981). In dog kidneys similar results were obtained with the exception that the total adenine nucleotide content started to decrease after 72 hours of machine perfusion (Southard,1984). It is unknown whether these differences are related to the use of different perfusate mediums. These data suggest, however, that the renal tissue is capable to maintain high tissue levels of adenine nucleotides by using endogenous substrate stores for at least 48 hours.

\section{Intervention studies}

Many pharmacological compounds have been used as additives to the perfusate to preserve the energy metabolism of the kidney during storage. Inosine has been supplemented as a direct precursor for nucleotide resynthesis. Improvement of postischemic function has been reported with and without a concomitant increase of the nucleotide levels (Buhl,1977; Fernando,1976). The usefulness of supplying nucleotides or nucleotide precursors has therefore been doubted. Other interventions have been proposed to prevent nucleotide exhaustion. Methylated xanthines are known to block effectively the break down of nucleoside monophosphates (Buhl,1979). Its possible beneficial effect on posttransplant function has to be explored. Using deoxycoformycin to block the break down of adenosine, together with addition of adenosine and phosphate, ATP levels can be enlarged to three times the original content. The mitochondrial function, however, is not improved with this technique.

Although there is no consensus about the most preferable technique in the clinical setting, some notable observations have been made in experimental studies. Life sustaining function of kidneys is maintained after 24 hours preservation both with simple storage following a flushing procedure and with machine perfusion using a standard perfusate medium. The differences are that the machine perfused kidneys function immediately upon implantation and recover more rapidly. After one month, however, both groups of kidneys function equally well (Gregg, 1986). The maximum length of preservation time with machine perfusion is higher than with cold storage. Canine kidneys can be safely stored with machine perfusion for 3 days. In a number of studies this limited time interval has been extended (Johnson, 1980;Rijkmans,1984) due to several modifications of the standard technique (Belzer,1967;Claes,1973). The limitations of the routine technique when applied for 3 days or more are not fully understood. Evaluation of mitochondrial function by determination of the respiratory control index and the tissue ATP content have failed to show alterations after prolongation of preservation time from 3 to 5 days (Southard,1984). The loss of effectiveness of machine perfusion after prolonged 
storage might result from a reduced maintenance of cellular fluid and ion homeostasis. Following 5 days of continuous hypothermic perfusion the capacity of the renal cells to re-accumulate potassium is markedly impaired (Southard,1984).

\section{Recovery of the energy metabolism following storage}

In conclusion, the level of energy metabolites is maintained during cold storage at near normal levels for a certain period of time. The final outcome appears to be species dependent and influenced the preservation technique used. There is evidence that machine perfusion extends this period and is able to maintain high levels of ATP and ADP. The question to examine now is what effect depressed tissue levels of energy metabolites such as ATP, ADP and others have on the recovery of the cellular energy metabolism following implantation. In canine kidneys stored for 24 and 48 hours on ice no increase of the nucleotide pool is observed during 60 minutes of reperfusion after implantation. The high levels of AMP at the end of cold storage, however, are rapidly converted to ATP and ADP. The energy charge after 60 minutes reperfusion is in the same range as in control tissue (chapter 7). Unfortunately, no data are available on differences in recovery rate between machine perfused and simply on ice stored kidneys. Studies on the metabolic properties of renal cortical tissue slices in vitro that were obtained from kidneys after prolonged machine perfusion, showed that renal cells were able to maintain steady state ATP levels after 3 days as well as after 5 days of storage (Southard,1984). Lack of further information makes it difficult to give a final statement on the relationship of cellular injury to the energy metabolism by hypothermia and the ability of the energy metabolism to recover following reinstallment of normoxic conditions. It is likelly that the length of preservation time, within reasonable limits, does not influence the restoration of the nucleotide pool early after the onset of reperfusion. Similarly, the capacity to rephosphorylate ATP from the available substrates is independent of preservation time (chapter 7, chapter 10).

It was recently argued that changes in the tissue adenine nucleotide content between the end of preservation time and 60 minutes of reperfusion may be substantially influenced by the effect of the so-called second warm ischemia time which occurs during from the implantation procedure (chapter 10). The paradoxical effect has been observed that kidneys with high levels of nucleotides just before implantation show a marked reduction of the nucleotide pool during implantation whereas this reduction does not occur in kidneys in which the nucleotide pool has already been depressed before implantation. This observation is explained by the dependency of the rate of nucleotide breakdown on the actual level of nucleotides in the tissue as discussed before.

\section{Prior warm ischemic injury}

A special problem in organ preservation is offered by the storage of kidneys that have previously suffered from warm ischemic injury. The maximum length of adequate preservation time is markedly reduced along with the extent of prior induced ischemic injury. In terms of energy metabolism, this may be translated in preservation of nucleotide levels, starting from a point at which depletion of nucleotide levels already exists. One might expect that this will lead to a further depletion during storage resulting in very low tissue levels. This, however, is not the case as appeared from studies in canine kidneys during simple cold storage. After 72 hours storage following flushing with Eurocollins 
the tissue adenine nucleotide content is similar in kidneys subjected to either zero, 30 , or 90 minutes of preceding warm ischemia ( chapter 5). The way in which these levels are reached, however, is markedly different. Nucleotide levels in kidneys with 90 minutes of warm ischemia time do not further decrease during 72 hours of cold storage. After 30 minutes of warm ischemia time nucleotide levels are initially preserved but drop between 24 and 48 hours of storage. In kidneys not subjected to ischemia part of the nucleotide content is lost during the flushing procedure but a second loss is only observed after 48 hours. Hence, kidneys proof to be able to maintain a nadir level of adenine nucleotides during hypothermia after some period of time. The time interval after which this nadir level is reached is determined by the level of tissue nucleotides at the beginning of the storage period. These findings substantiate previous observations on kidneys with or without 20 minutes of warm ischemia before cold storage in a different storage solution (Southard,1977). Further evidence to the existence of nadir levels of tissue nucleotides during storage have also been reported in studies on prolongation of storage time to 120 hours (Fischer,1981).

If the integrity of the energy metabolism in these kidneys at the end of storage is different this is not readily apparent from changes of tissue nucleotide content early during reperfusion following implantation. Generally, the nucleotide content at the end of 60 minutes reperfusion is not different from the nucleotide content at the end of the storage period suggesting that the cells are not able to replenish their nucleotide pool. Notably, following warm ischemic injury without cold storage the capacity to replenish the nucleotide pool is maintained even after 90 minutes of ischemia. Generation of ATP from the available nucleotides such as ADP and AMP is obviously not hampered. In kidneys with and without warm ischemia before 24 and 48 hours cold storage ATP rephosphorylation is observed. Similarly, rephosphorylation in kidneys with 90 minutes of warm ischemia time and 48 hours cold storage is not worse than in kidneys with only 30 minutes of warm ischemia time and 48 hours cold storage (chapter 7 and 10 ).

Hypothermic machine perfusion of ischemically damaged kidneys is thought to be superior to simple cold storage. In canine as well as in rabbit kidneys it has been found that tissue nucleotide levels do not decrease during 48 hours machine perfusion after prior exposure to warm ischemia during 0 to 60 minutes. It is even possible to increase the adenine nucleotide content during hypothermic machine perfusion in kidneys with a severely depleted nucleotide pool after 60 minutes of warm ischemia by using adequate modifications of the perfusion solution. High oxygen tensions and the addition of hypoxanthine as nucleotide precursor, restore nucleotide levels within 48 hours to preischemic levels. The possible beneficial effect of these changes on posttransplant tissue energy metabolism has not been examined (Pegg,1981;Pegg,1984).

\section{VIABILITY ASSESSMENT}

In 1972 an important hypothesis about viability assessment in kidneys using changes in adenine nucleotide levels was proposed by K.C.Calman. Most studies on the possible relationship of adenine nucleotide metabolism and renal viability refer to this hypothesis. Calman assumed that the viability of an organ, after storage, correlates to the content of total adenine nucleotide in the organ. He failed to verify his hypothesis but others tried ever since. His choice for tissue adenine nucleotide levels as the most promising parameter of viability assessment arose from the general idea that persistent ischemia stops 
oxidative phosphorylation and hence, causes a decrease of adenine nucleotide content with subsequent changes of cellular integrity as discussed before. Calman emphasized the need for a theoretical fundament for future viability studies but he was certainly not the first one to recognize the potential usefulness of the adenine nucleotide metabolism in this respect. Others had already described changes in tissue levels of purine compounds along with ischemia time in kidneys and had recognized some typical features that were related to the apparent ischemia tolerance of kidneys. Even efforts to use these findings for evaluating postischemic function had been published before. Calman, however, was probably the first who tried to rationalize this approach and made explicit some demands for the reliability of viability parameters that are still accepted to be crucial today.

\section{Adenine nucleotides}

The use of ATP as a single marker of viability is most attractive. ATP as the final "transmitter of energy' for all chemical reactions in the cell, is easy apprehensible as a prerequisite for the maintenance of cellular viability. In kidney, the study of the relationship between ATP levels and the extent of ischemic damage presents some special difficulties. In fact, it has been demonstrated that measurements of tissue ATP levels are not suitable for assessing the viability of renal tissue without taking into account the conditions in which the measurements have been made. The value of ATP as a viability marker depends on the assay technique as well as the circumstances in which the measurements were performed such as normothermic ischemia, cold storage, or reperfusion (chapter 4,5, and 9).

At least three approaches to the use of changes ATP and in adenine nucleotide levels in viability assessment can be distinguished based on the assumptions that either (1) a particular level of tissue nucleotides marks the transition from reversible to irreversible damage, or (2) the rate of nucleotide resynthesis following reperfusion indicates the viability of an organ, or (3) the changes in the tissue content of nucleotides correlate to the length of ischemia time.

\section{Point of no return}

(1)The most optimistic approach is represented by the search for a critical level of ATP or adenine nucleotides below which the renal energy metabolism will be unable to recover from ischemic damage. Such a point of no return has not been established (Bore,1979). This point may occur after a period of ischemia which exceeds the minimum period of ischemia time that will turn the kidney to a non-viable state. In vitro studies using cultured renal cells, have also failed to discriminate a point of no return. Lowering ATP levels with the use of the mitochondrial inhibitor antimycin does not reveal a critic ATP level. ATP levels can be brought down to non-measurable quantities without leading to a concomitant loss of membrane integrity (Kreisberg, 1986; Shapiro, 1986).

\section{Recovery rate}

(2)Monitoring the postischemic, or postimplantation, recovery of depleted adenine nucleotide levels is an obvious next approach. Whatever the changes of the levels of energy metabolites during ischemia are, if following reinstallment of normal oxygen and sub- 
strate delivery the cellular energy metabolism does not recover, cells will inevitably die. It has therefore generally been assumed that the lack of replenishment of the adenine nucleotide store is a final proof of actual damage to the cell. This method has a disadvantage to assess renal viability in the clinical setting as it can only be performed after the, possible non-viable, kidney has been transplanted. By assessing the extent of preexisting ischemic damage it may help in the diagnosis of early posttransplant non-function. In animal studies this method has revealed useful information on the role of adenine nucleotide levels to the functional state of the kidney. The rate as well as the extent of ATP recovery in rat during the first hours of reperfusion has been found to be dependent on the length of ischemia time varying from 30 to 180 minutes at $37^{\circ} \mathrm{C}$. Notably, some ATP recovery was observed even after 180 minutes of ischemia (Tatsukawa, 1979). Periods of warm ischemia between 15 and 60 minutes correlate to postischemic inulin clearance in rat. The increase of ATP upon reperfusion, similarly, correlates to the preceding ischemia time and the inulin clearance 24 hours following implantation (Cooper,1986). After 10 minutes of reperfusion following various intervals of ischemia non-functioning rat kidneys have lower ATP levels than functioning kidneys. However, no ATP value has been discriminated which predicts lack of functional recovery (Bore,1979). In isolated perfused rat kidneys no increase of total adenine nucleotide levels is found within 60 minutes of reperfusion after either 30,60 , or 90 minutes of ischemia. ATP levels, however, considerably increase at a similar rate after 30 and 60 minutes of ischemia. The ATP recovery after 90 minutes of ischemia is negligible (Shapiro,1986). Following in situ reperfusion after 30 and 90 minutes of ischemia in dog, the increase of adenine nucleotides after 5 and 60 minutes corresponds to the ischemia time (Maessen,1987a). Following 24 and 48 hours cold storage no increase of adenine nucleotides are observed within 60 minutes posttransplant reperfusion with or without prior exposure to warm ischemia (chapter 10). These data suggest that there is some relationship between the extent of ischemic injury, renal functional recovery, and the capacity to recover ATP and to replenish the nucleotide pool. The discriminating properties of ATP values apparently depend to reperfusion time.

\section{Effect of interventions}

Contradictory data about these relationships are obtained in intervention studies. Infusion of the nucleoside inosine in kidneys before they are rendered ischemic for 90 minutes improves the endogenous creatinine clearance following reperfusion. This treatment, however, does not increase the nucleotide levels early during reperfusion (Buhl,1977; Marschall,1978). Others found both an effect on functional parameters as well as on the ATP recovery rate (Fernando,1976). In the isolated perfused rat kidney postischemic inulin clearance and tubular sodium re-absorbtion are similar in kidneys with and without treatment with fructose and glycerol. The addition of these substances causes a threefold decrease of tissue ATP levels (Shapiro,1986). Supply of exogenous adenine nucleotides to isolated renal tubules has been found to induce a rise of the intracellular nucleotide pool to supranormal values (Weinberg,1986). Other nucleotide precursors are known to be toxic if infused in combination to exposure to hypothermia (Buhl,1977). A beneficial effect on both renal function and levels of adenine nucleotides has been obtained by kidney pretreatment with the calcium channel blocker verapamil. A doubling of the ATP content as well as total adenine nucleotide content results from 
verapamil treatment within 60 minutes of reperfusion following 40 minutes of ischemia in rat (Shapiro,1985). Addition of ATP-MgCL 2 improves postischemic renal bloodflow and the recovery of tissue ATP. The increase in renal bloodflow persist whereas treatment with dopamine induces a transient increase in bloodflow and no improvement of ATP levels suggesting that the beneficial effect of ATP-MgCL 2 depends also on the concomitant increase of ATP (Gaudio,1986). With respect to these findings it is important to note that early posttransplant function may not correspond to function after a prolonged period of time. This relation strongly depends on the preservation technique used (Gregg,1986;Gaudio,1986). So until now there is no consensus as to whether the recovery of the adenine nucleotide metabolism is indicative for ischemic injury or organ viability. The correlation which has been found in a simple experimental in situ ischemia model is lost if additional influences are introduced.

Time-course relationship

(3)In a third type of studies a more pragmatic approach has been chosen. The relationship of adenine nucleotide loss and ischemia time is considered as a temporal relationship. The assumption underlying these studies is that the length of ischemia time correlates to postischemic viability. The fundamental question as to whether the tissue ATP or adenine nucleotide level is causally involved in the development of irreversible damage is more or less neglected.

The pattern of ATP loss which is characteristic for renal tissue does not show a relationship with the length of prolonged periods of ischemia time as discussed previously. A better correlation to ischemia time has been obtained by monitoring changes of the total adenine nucleotide pool. This pool gradually decreases along with the length of ischemia time (Bergstom,1970). Small changes of temperature have to be taken into account as they may considerably influence the rate of adenine nucleotide break down ( $\mathrm{Za}$ ger,1986; chapter 4). Recently, it has been shown that a reasonable estimation of warm ischemia time can be made by the assessment of the balance between the adenine nucleotides and their degradation products. This method has been developed to determine warm ischemia time in donorkidneys at the end of nephrectomy (chapter 4). Early experiences in the clinical setting have been reported (Maessen,1988c)

Viability assessment during hypothermia

Nucleotides as a viability parameter during hypothermic storage of kidneys have been studied in the same way as during normothermic ischemia. Due to the retardation of the breakdown of e.g. ATP by hypothermia one might expect a more useful time-course relationship of nucleotide loss and length of preservation time. This concept appears incorrect from a practical point of view. Current hypothermic storage techniques introduce a complicated set of changes in the physiological conditions of the organ that are far more heterogenous than the conditions that are induced by experimental normothermic ischemia. In the most simple concept of hypothermic storage, i.e. immediate surface cooling of the freshly excised kidney using melting ice, the rate of ATP loss progressively decreases. After 30 minutes the decrease of the ATP levels is still similar to the decrease during 30 minutes normothermic ischemia. After 120 minutes ATP levels in hypothermic tissue are fourfold higher than in normothermic tissue. It should be noted that these values are less than $10 \%$ of normoxic control values. Until now a simple slow 
down of the events during normothermic ischemia is a plausible explanation. Changes to this primitive preservation method, have all shown to affect more or less the characteristic changes of the ATP concentration.

It has been suggested that adenine nucleotide levels give more information about the effect of hypothermia than only the level of ATP since the rate of adenine nucleotide break down is constant during cold storage (Warnick,1977; Beck,1977; Buhl,1976). Other observations however indicate that adenine nucleotide levels reach nadir levels in catabolic conditions. Furthermore, during hypothermia adenine nucleotide levels only drop after being kept constant for some period of time (chapter 10). Flushing of the excised kidney has been introduced among others to accelerate cooling of the tissue. Unfortunately, this procedure is not standardized since the ideal flushing time, pressure, temperature, and the composition of the flush solution are still subject to investigations. The increased cooling rate by flushing at $4^{\circ} \mathrm{C}$ effectively improves the slow down of the ATP break down as after 8 minutes the ATP levels are approximately twofold higher than in normothermic ischemic control kidneys. Both an increase and a decrease of adenine nucleotide levels following flushing have been reported (Kallerhof,1987; chapter 10). Monitoring a time-course relationship during hypothermia does therefore only make sense if the effect of flushing on the nucleotide levels is known. A direct relationship of adenine nucleotide levels and renal viability in these conditions remain obscure. The increase of the cooling rate does not guarantee improved posttransplant viability. For some unknown reasons rapid cooling techniques may even hamper renal recovery following implantation, a phenomenon which has been described as "cold shock".

Prior warm ischemic injury

Warm ischemic injury to kidneys before storage hampers a straightforward interpretation of adenine nucleotide values. The adenine nucleotide loss during simple storage on ice initiated by flushing is dependent on the level of adenine nucleotides before the procedure is started (chapter 7). High levels of nucleotides correspond to a high loss of these substances during flushing as well as during the subsequent storage period. Prior lowering of nucleotide levels by a period of normothermic ischemia results in a more slowly break down during preservation of the amount of nucleotides which is left. Therefore, after some time during the preservation period nucleotide levels will be similar in kidneys with or without preexisting irreversible ischemic damage. During simple cold storage adenine nucleotide levels may indicate prior warm ischemia time if the length of preservation time is known and less than 24 hours (chapter 10).

Nucleotide levels gradually decrease during hypothermic continuous perfusion. Addition of sufficient amounts of adenosine and phosphate to the perfusate medium induces ATP synthesis during hypothermia to such an extent that after three days supranormal levels are reached. This effect is markedly enhanced if simultaneously adenosine deaminase is blocked by deoxycoformycin. The ATP synthesis continues up to 5 days hypothermic perfusion when the viability of the organ is already lost if tested in a unilateral transplant model. Despite 60 minutes of warm ischemia it is possible to raise the tissue adenine nucleotide content during machine perfusion with modified perfusates, considerable. Unfortunately, these changes do not automatically improve posttransplant function and viability (Pegg,1984). On the other hand a remarkable improvement of posttransplant viability of ischemically damaged kidneys has been obtained by retrograde 
oxygen persufflation without changes to the tissue nucleotide levels (Rolles,1984). Furthermore, pretreatment of canine kidneys with ATP-MgCL 2 before storage shows a better conservation of ATP levels than without treatment. In contrast, posttransplant survival is better in kidneys without treatment (Garvin,1982). The effects of different preservation techniques is strongly species dependent (Fischer,1979). Obviously, the capacity of the renal tissue to synthesis ATP is preserved whereas an artificial increase of nucleotide levels does not affect viability. The question is therefore raised whether a need for exogenously increased ATP levels is justified.

These data exemplify the difficulty to assess the importance of nucleotide levels during hypothermic storage as the actual nucleotide level is determined by the preservation techniques that may or may not influence posttranplant viability of the kidneys. The use of adenine nucleotide levels at the end of the preservation time to evaluate the efficacy of preservation techniques seems not suitable as long as a possible effect on posttranplant viability has not been determined.

\section{CONCLUDING REMARKS}

For some reasons which have been discussed before a relation between tissue adenine nucleotide levels and postischemic viability of kidneys has been assumed. According to studies performed in the forties and fifties, showing a remarkable ischemia tolerance of the kidney and to the analysis of the characteristic break down pattern of purine metabolites in kidneys which were considered responsible for the relative insensitivity to ischemia in the early sixties, a correlation was postulated between changes of the renal energy metabolism and viability. From that time onwards investigators, especially in the field of organ transplantation, have tried to employ this putative relationship for evaluation and improvement of their techniques. More recently, a steadily increasing number of papers have been published in which more basic aspects of the energy metabolism and pathological renal function have been studied.

The original assumption that a direct relationship exists between tissue nucleotide levels and organ viability, has hampered to rate later findings regarding this subject on their true value. Such a misconception might be that low adenine nucleotide levels during and following ischemia are a symptom of distress and need to be corrected. Until now there is no conclusive evidence from intervention studies that increased nucleotide levels indicate improved function and vice versa. By contrast, it is highly surprising to see that the important process of coupling energy production to consumption is well preserved during prolonged ischemia. This coupling seems to exist much longer than the period after which the kidney is known to have lost its life sustaining function. Therefore, it may be alternatively assumed that the homeostasis of the energy metabolism is the most powerful and ischemia tolerant part of the cell and, paradoxically, that it remains intact as long as the cell is alive.

Although in this view there is no causal relation between the fall in the level of high energy metabolites and the loss of viability of the ischemically damaged cell, a time-course relation between events taking place in the energy metabolism and the length of ischemia time may be present. Such a relationship justifies the use of adenine nucleotide levels in viability assessment under certain conditions. This relationship is most reliable in normothermic ischemia between 0 and 90 minutes. Determination of purine metabolites is useful to estimate warm ischemia time when determination is performed in normother- 
mic kidneys. During hypothermia this relationship may be only practicable for comparison of results when using the same preservation techniques. 


\section{CHAPTER 2}

\section{QUANTITATION OF ISCHEMIC, STRUCTURAL RENAL CELL DAMAGE}

\section{INTRODUCTION}

In the previous chapter a method has been discussed to monitor processes at a metabolic level that may lead to the development of irreversible ischemic injury. A different approach to the evaluation of ischemic tissue injury, however, is the measurement of cell damage which has already been established. The purpose of this approach is not to detect the mechanisms that will eventually be responsible for cell damage, but to estimate the actual amount of tissue that has lost viability. Both approaches may be valuable through different ways for the prediction of the outcome of postischemic renal recovery. A most prominent feature of the dying cell is the loss of structural integrity. Normally, membranous coupled mechanisms and structures offer an effective means to create and preserve the intracellular homeostasis of the viable cell. An early symptom of the cell in distress is an increase of membrane permeability which weakens the resistance of the cell to the surrounding environment. A derangement of the Na-K pump either by inadequate energy supply or direct loss of function of the Na,K-ATPase complex is suggested from a rapid loss of the sodium/potassium balance. Consequently, a shift in the transport of ions from and to the cell occurs in association to a change of the membrane potential (Hochachka,1986). These relatively small changes of membrane permeability may lead through a cascade of events including disturbances of the intracellular calcium homeostasis, phospholipase activation, acidification, and a progressive swelling of the cell, to gross membrane disruption (Chaudry,1983). The initiating cause of disturbances in membrane integrity often remains obscure. Some mechanisms have been discovered that play a pivotal role to the increase of membrane permeability in cell dying. The cellular membrane has been found to be the main target for a large number of biological toxins. Complement and cell mediated cytolysis are generally considered to evolve from the formation of tubular pore holes in the membrane (Young,1987). Further, a heavy osmotic challenge may surpass the capacity of the membrane to withstand stress leading to cell swelling and membrane disruption (Billah,1976). Finally, endogenous intracellular processes have found to be able to initiate the derangement of the membrane, e.g. in processes like ageing and embryonic development (Duvall,1986).

In ischemia induced cell damage, alterations in membrane integrity are thought to be involved in the eventual decline of cellular viability (Hochachka,1986). The cause of the membrane damage has been generally associated with the development of a discrepancy between energy demand and energy supply. Lack of oxygen causes an arrest of mitochondrial oxidative phosphorylation and hence a decreasing ability to deliver ATP. Alternative energy yielding processes may only temporarily meet the demand of the cell. Despite mechanisms to increase ischemia tolerance by lowering the cellular energy need, shortage of ATP will eventually limit the ability of the cell to maintain internal homeostasis during prolonged ischemia. This attractive hypothesis is not completely satisfactorily. Some recent observations suggest that the effectiveness of secondary adaptive 
mechanisms to ischemic conditions subsides and lead to irreversible blebdamage whereas the recovery capacity of the energy metabolism remains relatively well intact (Weinberg,1988). The oll hypothesis that cells die from the lytic effect of lysosomal enzymes that are normally safely encapsulated within the cell has lost interest (Reimer,1984). These enzymes are only observed after gross structural damage has already been established. A closely related concept, however, is still worth to be considered. During sustained ischemia metabolic intermediates may accumulate to such an extent that they become toxic (McCord,1985). There is some evidence that these compounds may directly interfere with membranous integrity. Furthermore, the final extent of lethal damage by ischemia in vivo may in part evolve from extracellular mechanisms in response to the ischemia-induced damage of the cell. Although, postischemic reperfusion is the only way to save the ischemic tissue, a number of damaged cells may be unable to withstand the renewed environmental conditions induced by reperfusion and die after all (Cheung, 1986; Henson,1987). Whatever the sequela of events is that leads to the membrane disruption, the increased permeability of the membrane will allow macro-molecules to leak into the cellular environment. It is generally assumed that the loss of macro-molecules is associated with severe cellular damage and cell death (Reimer,1984;Hermens,1982).

\section{ENZYMES AS MARKERS OF TISSUE DAMAGE}

If it is now assumed that proteins which get lost from the disintegrated cell are transported to body fluids, e.g. into the blood, the extent of ischemic injury might be evaluated by the assessment of these proteins. Since methods to determine the plasma activity of tissue enzymes are available from the beginning of our century (Wohlgemut,1908) these proteins have received much attention to trace tissue damage. Especially in case of liver and heart injury clinical application of enzyme assay techniques have been extensively worked out.

The use of enzymes as diagnostic parameters in respect to renal damage has never become very popular (Dubach,1970). Acute renal ischemic damage is mainly encountered in the setting of renal transplantation. This field got a strong impetus when only recently techniques became available to obtain reliable assessment of enzyme activity in the urinary fluid (Raab,1972; Price,1982). Normal urinary enzyme activity is low and changes are with minor exceptions attributable to the kidney. Small changes are readily noticed and offer thus a sensitive indicator of renal damage. In contrast, changes in plasma enzyme activity due to renal damage have been learned to be small and difficult to discriminate from other, non-renal sources of enzyme loss (Mattenheimer,1977). The major value of urinary enzyme diagnostics is the ability to signalize changes in renal enzyme handling (Vanderlinde,1981). In kidney transplantation urinary enzyme assays have been useful in obtaining an early warning of rejection before functional disturbances become measurable (Bourbouze, 1985). In studies on nephrotoxicity of drugs highly specific information on the primary site of action of the drug can be obtained by calculating the ratio of enzymes appearing in urine (Price,1982). In these studies generally membrane bound enzymes are considered that are located at the luminal site of the tubular epithelium. The appearance of cytosolic enzymes in urine has also been demonstrated albeit with less conclusive results (Prout,1964).

Following ischemic renal injury changes of both plasma enzyme activity and urinary 
enzyme activity have been reported in experimental studies (Mattenheimer 1977). These changes allowed to detect damage to the kidney in a qualitative way. However, the diagnostic usefulness of the assessment of renal enzyme activity would be markedly improved if it is possible to obtain a quantitative determination of the amount of enzyme activity that has been lost from the kidney. With this information the extent of ischemic injury might be estimated. This information may be obtained by direct determination of changes in tissue enzyme activity. This is only practicable in experimental studies as relative large amounts of tissue are needed to obtain reliable results (Hermens, 1982). Indirect measurements of enzyme loss, considering changes of enzyme activity in body fluids is obviously advantageous. The question therefore is, whether it is possible to assess the quantitative amount of renal enzyme activity that is lost from damaged tissue by measuring enzyme leakage in plasma or in urine.

\section{ESTIMATION OF INFARCT SIZE}

Attempts to achieve this goal have been performed in studies on myocardial infarction (Shell,1972;Roberts,1975). Estimations of myocardial infarct size have been successful by using information from changes in plasma enzyme activity. Processing of these data with a mathematical model concerning the kinetics of enzymes in the body fluid compartments has learned that the amount of enzyme activity entering the circulation during some period of time can be reliably calculated (Willems,1985). Experimental studies have shown a good correlation of the amount of enzyme activity in plasma which has been calculated in this way and the loss of enzyme activity from the myocardial infarcted area. This method is routinely used in clinical myocardial infarct size estimation, especially in order to evaluate the effect of therapeutic interventions (Willems,1987).

There are, however, a number of important differences between the situation in the heart and in the kidney that may complicate the use of this method for the assessment of the extent of ischemic injury in kidneys. The mixed cell population which constitutes the kidney may be responsible for a number of variations that are not encountered in organs with a homogeneous cell distribution. Furthermore, in kidney enzymes may drain off to the urine as well as to the circulation whereas in heart tissue only the latter possibility is open. The choice of marker enzymes of renal enzyme loss is difficult as no obvious renal specific enzymes are known that might be representative for general renal damage. More generally, a number of assumptions are made in the application of the quantitation model that still need to be verified in case of kidneys. For instance, it is possible that the extent of ischemic injury that causes a decline of renal viability does not affect the average renal tissue enzyme content. Highly specific lesions to particular structures of the nephron that impair postischemic renal recovery may precede gross structural tissue damage and enzyme loss (Mason,1987). In order to examine the relation of tissue enzyme loss and recovery of enzyme activity in body fluids the postischemic residual renal enzyme content has to be determined. However, the renal tissue is known to have a remarkable regenerative capacity following ischemic damage. Hypertrophy as well as hyperplasy may hamper the assessment of the residual tissue enzyme content following some period after the ischemic insult. In the next paragraphs these and other characteristics of renal enzyme behaviour will be discussed. 


\section{TRANSPORT OF ENZYMES FROM THE KIDNEYS TO PLASMA OR URINE}

It has been found in heart tissue that cellular substances that enter into the extracellular space are recovered in plasma after some time (Hermens, 1982). As an important aspect of the renal function concerns the regulation of the transport of substances from the plasma compartment to the urine and hence the outside of the body, it is amenable that part of the enzyme activity that is lost from damaged cells enters into the urinary system. Most cells of the renal tissue face on one side of their border the lumen of a tubule. On the other side they are embedded in interstitial fluid. Both enzyme loss to plasma and urine have been observed. It is unknown however in which proportion enzymes either leak into urine or plasma (Donohoe,1978). Recently studies were undertaken to examine this point (chapter 11). Purified enzyme preparations were infused into the cortical renal parenchyma in order to mimic a accumulation of free enzymes. Since a known quantity of enzyme activity was infused in a period of 60 minutes the possible recovery in plasma or in urine could be calculated. Within one hour after the start of the enzyme infusion approximately $80 \%$ of the infused enzyme was recovered in plasma. Determination of enzyme activity in urine samples that were continuously collected by cannulating the renal ureter showed that within two hours less than one percent of the infused enzyme had been excreted into the urinary system. These rather surprising results might have been established by the fact that the infusions were performed in non-damaged tissues. Therefore, the same experiments were repeated in kidneys that had previously lylbeen rendered ischemic by ligation of a single renal artery branch. As a result about half of the kidney is devoid of arterial blood supply. Infusion in this ischemic area, however, showed a similar distribution of enzyme activity to plasma and urine within the same time as in non-damaged tissues. Doubling of the infusion rate did not alter the intrarenal pressure nor the enzyme recovery rate. Hence, the rapid recovery in plasma was unlikely to result from a pressure artefact. Since in these experiments there was no arterial circulation to the ischemic area the existence of some kind of interstitial fluid circulation has to be assumed (Szabo,1978). A similar phenomenon has been suggested from observations of enzyme transport from infarcted areas in the myocardium (Hermens,1982). These studies in the dog suggest that the release of enzyme activity from renal tissue leads to accumulation of this activity in plasma and not in urine. Furthermore, it is suggested that the time lag between the entrance of enzymes in the extracellular space and the recovery in plasma is in the range of order of minutes to hours. One might therefore reasonably expect that if enzymes from damaged cells leak into the extracellular space they are rapidly transported to the blood circulation. Later studies were in agreement with this assumption (chapter 11 to 13).

\section{CHANGES IN TISSUE ENZYME ACTIVTTY}

The next question that has to be answered is whether ischemic injury leads to measurable changes of renal tissue enzyme activity. To be able to relate the cumulative amount of enzyme in plasma to the tissue enzyme loss, changes in tissue enzyme activity need to be large enough to be detected. Furthermore, the residual tissue enzyme activity following infarction can only be accurately assessed if the normal, non-ischemic tissue content shows a relative small individual variation.

With respect to the subject of our study some particular types of renal ischemic injury are relevant. Before donor nephrectomy and during the time in between donor nephrec- 
tomy and the onset of preservation a transient global ischemic insult may occur. $\mathrm{Hy}$ pothermic preservation may present a second type of global ischemic injury whose nature is not precisely known (Pegg,1986). During implantation, again, a global ischemic insult may be induced as temperature is increased during this procedure without reestablishment of a normal blood circulation. Finally, following reperfusion of injured tissue, occlusion of intrarenal vessels may occur leading to permanently infarcted areas within the kidney (Mason,1987). In recent studies, the effect of a number of these possible damaging insults on the renal tissue enzyme activity were documented (chapter 13). In dog, a panel of enzymes was studied including cytosolic enzymes (LDH, and AST), the mitochondrial enzyme GLDH, and brush border enzymes (AP, and GGT). Considerable differences in concentrations of these enzymes exist between cortical and medullar tissue. In the latter the concentration of cytosolic enzyme activity is about half the concentration in the cortex. The content of the mitochondrial GLDH is $25 \%$ of the cortical content whereas the activity of the brush border enzymes is only $10 \%$. The coefficients of variation of the tissue enzyme activity in individual kidneys ranges from 10 to about 20\%. This value is only $7 \%$ for AST and hence allows the use of an average control value for tissue AST activity. Similar variations have been reported for human kidneys (Dubach,1970). Ischemia has a profound effect on tissue enzyme activity. After 90 minutes of obstruction of the renal blood supply and 48 hours reperfusion in situ the average enzyme content is reduced to about $60 \%$. By contrast, after 30 minutes of ischemia an average tissue enzyme content of $90 \%$ is maintained. If the ischemic injury is sustained by permanent ligation of a single branch of the renal artery a depletion of the ischemic tissue enzyme content within two days is observed of about $50 \%$. In experiments in which kidneys were stored on ice with or without prior exposure to ischemia it has been found that even short term preservation ( 24 hours) causes a considerable additional depletion of enzyme activity during reperfusion following implantation (chapter 12). In the above mentioned conditions the loss of enzyme activity is generally more pronounced in the cortex than in the medulla. As the cortical tissue comprises about $80 \%$ of total renal mass, the differences that have been noticed are largely determined by changes in the cortical enzyme content. It is thus shown that a general and graded depletion of the renal cytosolic and mitochondrial enzyme activity is observed as a result of damaging conditions which may be encountered during renal preservation and transplantation.

\section{ENZYME ACTIVITY FOLLOWING ISCHEMIC RENAL INJURY}

According to a number of previous reports a general opinion has arisen that changes of plasma enzyme activity due to renal damage are hardly significant and difficult to detect (Mattenheimer,1977). Recent experimental observations have shown that this only in part holds true (chapter 12). Following postischemic reperfusion of canine kidneys a clear and significant rise in plasma of a number of tissue enzyme activities is found ranging from a 3 to 6 fold increase of normal plasma levels. However, these changes are only detected by frequent sampling early during reperfusion, i.e. within a few hours. Following 90 minutes of occlusion of the renal vascular supply peak values of cytosolic enzyme activities (LDH, and AST) in plasma are found within 2 to 4 hours. After 48 hours of reperfusion plasma levels have generally normalized. Notably, mainly levels of the cytosolic enzymes are increased despite a general depletion of the tissue enzyme content, in- 
cluding mitochondrial and brush border enzymes (chapter 13). Obviously cytosolic enzymes freely move to the interstitium as soon as the cell membrane becomes permeable to macro-molecules. Enzymes that are more closely bound to intracellular structures may only be released after some delay. In case of mitochondriall GLDH such a mechanism is suggested from studies on sustained renal ischemic damage. Following permanent occlusion of one half of the renal arterial blood supply a small increase of plasma GLDH activity is observed some hours after the increase of cytosolic enzymes is started. These results are in agreement with previous findings in studies on myocardial infarction. It has been suggested that the appearance of mitochondrial enzymes in plasma is associated with severe necrosis (Hermens, 1982).

\section{CUMULATIVE AMOUNT OF PLASMA ENZYME ACTIVITY}

Since a reasonable recovery of enzyme activity in plasma in relation to renal ischemic injury occurs and can be detected, methods to quantitate the recovery in plasma can be applied. In previous studies a number of mathematical formulas have been designed and tested on the basis of a two-compartment model for circulating proteins (Willems,1985). If enzymes are released from tissues they will, within a particular period of time, be distributed to the intravascular compartment as well as to extravascular compartments. A steady exchange of enzymes between these compartments may be assumed. In addition to the influence of these kinetic factors enzymes are broken down at a certain rate during the same time. Previous studies have shown that there is generally no significant extravascular breakdown of circulating enzymes (Hermens, 1982). Hence, only the intravascular breakdown of enzyme has to be considered. Thus, the cumulative amount of enzyme released up to time $t$ per litre of plasma, $Q(t)$, is represented by $Q(t)=C(t)+E(t)+F C R . o \int^{t} C(t) d t$

in which $C(t)$ presents the enzyme activity in plasma at time $t$ (corrected for the normal steady state activity), and $\mathrm{E}(\mathrm{t})$ the extravascular enzyme activity at time $t$. The last term gives the enzyme activity which has been eliminated from plasma up to time $t$ (FCR is the fractional catabolic rate constant). The extravascular enzyme activity is calculated as:

$E(t)=T E R \cdot \exp (-E R R \cdot t)_{0} \int^{t} \exp (E R R \cdot t) C(t) d t$

Values of TER (transcapillary escape rate constant) and ERR (extravascular return rate constant) have previously been determined in the dog (Hermens,1982). Fixed values can be used for different enzymes. These calculations can therefore be performed in each individual animal if the plasma volume is known. An important parameter in these equations is the fractional catabolic rate constant which is specific for the individual enzyme used. The FCR of a large number of enzymes has been determined by monitoring the disappearance rate of the enzyme activity in plasma after a bolus injection of a purified enzyme preparation (Hermens,1982). Recently, similar studies were performed using enzyme preparations of fresh kidney homogenates (chapter 12). No gross differences were found between values obtained with these native preparations and with purified preparations. Besides being enzyme specific there is some evidence that FCR is influenced by the general condition of the organism. Further studies are needed to determine the importance of this effect.

Since the enzyme activities that were found to be increased in plasma following renal in- 
jury show important dissimilarities with respect to their individual fractional catabolic rate, comparison of the observed changes in plasma activity are misleading. Peak values in plasma enzyme activity may be largely determined by the half life of the enzymes in plasma. Furthermore, a sudden appearance of enzyme in plasma may results in a higher peak value than a more gradual appearance whereas the same amount of enzyme activity may have been released from the tissue. Eliminating these variations by calculating the cumulative release of enzyme in plasma per unit of time reveals a proper picture of the amount of enzyme activity that enters the plasma circulation during some period of time. With this technique it has been demonstrated that the cumulative release of enzyme activity in plasma depends to the nature and the severity of the ischemic insult (chapter 12). In canine kidneys that have been rendered ischemic for 90 minutes there is a large release of cytosolic enzyme into the plasma within the first few hours after the onset of reperfusion. During the next hours the amount of enzyme that enters the circulation is rapidly reduced. The bulk of renal enzyme is released within the first 20 hours following ischemia. Following renal infarction by permanent ligation of a single branch of the renal artery the cumulative release of enzyme is significantly different. Within the first two hours there is hardly any release of enzyme. Between 3 to 4 hours a gradual release is started which slowly rises up to 8 to 12 hours after the start of the infarction. Since previous studies have shown that enzymes which accumulate interstitially are rapidly transported to the circulation, although the tissue lacks arterial blood supply, it must be assumed that after 3 to 4 hours of permanent ischemia there is still very limited leakage of enzymes from the ischemic cells (chapter 11). The sudden release in the reperfusion studies is therefore unlikely to represent a wash out effect. It probably results from additional damage as induced during reperfusion. These data therefore provide evidence for the existence of a reperfusion injury phenomenon.

\section{TISSUE ENZYME LOSS AND PLASMA RECOVERY}

The values of the cumulative plasma enzyme activity can be expressed in gram equivalents of renal tissue by calculating the ratio of the total amount of enzyme activity in plasma and the concentration of enzyme activity in one gram of renal tissue of the corresponding enzyme. One might expect to find approximately similar values for all enzymes since the tissue depletion was found to be more or less the same for each enzyme. However, dependent on the nature of the ischemic injury differences have been found between the recovery of the individual amounts of enzyme activity when expressed in gram equivalents of renal tissue (Chapter 12). First, following 90 minutes of ischemia and subsequent reperfusion of canine kidneys no recovery of GLDH in plasma is observed (Maessen,1988p). In experiments with permanent ischemic damage there is some recovery of GLDH suggesting, when expressed in gram equivalents of renal tissue, the loss of enzyme content of a few grams of renal tissue. The concomitant recovery of cytosolic enzymes, however, suggests a much greater loss of renal tissue, up to one half of total kidney weight. Second, in experiments with permanent ischemic damage the cumulative amount of both LDH and AST activity expressed in gram equivalents of renal tissue indicate a similar extent of damage. Following 90 minutes of transient ischemic damage, however, the loss of gram equivalents of renal tissue is considerable reduced when calculated with LDH in comparison to AST. It is thus suggested that apart from being transported to the plasma and perhaps to a small extent to the urinary system, 
there are still other ways in which enzyme activity may get lost from the tissue. A number of possible mechanisms, like lymphatic drainage and local degradation, have been considered in the literature (\$zabo,1974).

The cumulative amount of enzyme activity in plasma following renal ischemia has been obtained in a chronically instrumented dog model. In this experimental model the release of enzyme activity to plasma from other sources than the kidney is avoided. In alternative models in which the effect of renal ischemia on plasma enzyme activity is studied during or immediately upon surgery errors are likely to occur. Enzyme loss from e.g. skeletal muscle is induced by surgical trauma and the effect of anesthesia. The additional enzyme loss may interfere with the putative enzyme loss from the ischemic kidney: Recent studies showed that such additional enzyme release can completely mask renal enzyme release (Maessen,1988p). The enzyme release from other sources than the kidney was characterized by a profound increase of plasma CK activity. Since renal ischemia does not induce a change in plasma CK levels, the activity of this enzyme was used to discriminate renal enzyme loss from non-renal enzyme loss. In animals after surgery without renal ischemia a ratio was determined between the increase of CK activity and the concomitant increase of cytosolic enzymes. In animals with both surgical trauma and renal ischemia this ratio was used to calculate the proportion of the cytosolic enzyme activity that contributes to the total change in plasma cytosolic enzyme activity, from nonrenal sources according to the increase of CK levels. Using this technique it appeared possible to distinguish the the renal AST activity from other sources of AST activity in plasma. Correction performed in this way for other enzymes were, however, moderately successful.

The final step in the efforts to quantitate the extent of ischemic injury using changes in plasma enzyme activity is to examine the relation between the cumulative amount of enzyme activity that is found in plasma and the loss of enzyme activity in the damaged tissue. Calculations of the cumulative plasma enzyme release of different enzymes already suggested that this relation depends on the enzyme marker that is used. Studies in dog have shown that the plasma recovery of cytosolic AST that is lost from the renal tissue after transient ischemic damage as well as during sustained ischemic damage is on average nearly complete (chapter 12).

Although it has been shown now that an estimation of the renal tissue enzyme loss due to ischemic injury is possible by measuring the cumulative enzyme activity in plasma there is still an important question to be aniswered. For a more fundamental interpretation of the information that is obtained using this method it is important to know what kind of tissue damage the enzyme loss reflects. This matter has been widely discussed without leading to a general opinion (Hermens,1982). In studies on hepatocytes leakage of enzymes have been observed without irreversible damage to the cells. In studies on heart tissue, however, the loss of enzymes is considered a symptom of dying cells (Willems, 1985;Reimer,1984). In kidney a similar mechanism has been assumed. Some new evidence, however, may arise from the above mentioned quantitative enzyme studies in combination to recently reported data on the response of the cellular energy metabolism to ischemic injury (chapter 13). In canine kidneys 90 minutes of ischemia induces a loss of about $40 \%$ of the renal enzyme content suggesting a similar percentage of cell death. The tissue adenine nucleotide content in these kidneys is also severely depleted in the order of $50 \%$. However, within one hour of reperfusion a small but clear increase 
of tissue nucleotide concentration is observed. This increase persists and within 24 hours tissue nucleotide levels are obtained that are comparable to preischemic values. Since the replenishment of the nucleotide pool obviously requires viable cells a $40 \%$ loss of viable renal tissue as suggested from the enzyme depletion is highly unlikely. Otherwise it must be assumed that a limited number of cells produce supra-normal nucleotide levels. However, there is no experimental evidence in support of this assumption (Weinberg, 1986; chapter 13). The discrepancy between the nucleotide and enzyme content after 48 hours of reperfusion may be explained by the fact that the restore of the normal cellular enzyme content is a more time consuming process. The functional recovery of these kidneys is extremely delayed and may be associated to the slow normalization of the cellular enzyme content. It may be concluded that renal cells following ischemic injury may loose part of their enzyme activity without being inevitably destined to die. 


\section{CHAPTER 3}

\section{INFLAMMATORY REACTIONS IN ISCHEMIC INJUAY}

\section{INTRODUCTION}

The parameters of ischemic renal injury that have been discussed in the previous chapters are thought to reflect cell damage mediated by inadequate oxygen supply and accumulation of metabolic waste products. The development of injury is considered an isolated process confined to events that take exclusively place within the cell and the intercellar connection (Kreisberg, 1980). This view seems to be justified as the ischemic conditions are produced by isolation of the organ from interaction with other organ systems. However, in a number of cases with obvious relevance to organ transplantation, justification of this concept may be questioned. After implantation the reinstallment of circulation renews the interaction of the kidney to other organ systems. From that moment onwards non-renal factors are involved in the modulation of the eventual outcome of tissue injury as well. Furthermore, when kidneys are either completely or partially rendered ischemic in situ, a similar influence of non-renal factors on the development of damage may exist. Such influence may play a role from the very beginning of the ischemic insult despite the lack of arterial circulation (Sheehan,1953). In this view, the final effect of ischemia should be considered as the sum of the initial disturbance of cell metabolism and the environmental response. The possibility that non-renal, or generally spoken non-target organ related factors exert an effect on the ischemic tissue has been recognized for many years. Since this effect has attracted attention because of an apparently negative influence on tissue recovery it has been defined as 'reperfusion injury'. The beneficial effect of reperfusion is obvious as it is after all the only way by which the ischemic tissue can recover. The concomitant occurrence of negative side effects, however, is puzzling and the true nature of the effect is therefore still under discussion. A number of observations have been reported in support of the existence of a reperfusion injury phenomenon including free radical damage, sustained ischemia by microvascular plugging, and, in heart tissue, calcium overloading. Some of these observations have been repeatedly associated with the accumulation of neutrophils in the infarcted area. The presence of these neutrophils suggests an inflammatory response at the site of the ischemically injured tissue.

In this chapter attention is focused on these inflammatory reactions to tissue ischemia. The involvement of the inflammatory response in the development of ischemic injury may have important implications to the evaluation of ischemic renal damage. First, the effect of the inflammatory response may be ignored by common viability tests as the effect becomes apparent after these tests have generally been performed. Second, the inflammatory response may correlate to the extent of the tissue damage and thus offer opportunities to monitor the infarct size. Third, if the mechanism of the inflammatory response is understood, adequate therapeutic interventions may be developed to relief the possible negative effect of inflammation on the extent of tissue damage. Finally, the working mechanism of previously used, infarct size reducing intervention strategies may have to be reconsidered if they appear to interfere with inflammatory mechanisms. 


\section{REPERFUSION INJURY}

There may seem to be some contradiction in the assumption that reperfusion may exert a negative effect on the outcome of ischemic tissue injury. The existence of a reperfusion injury phenomenon is therefore not generally accepted (Poole-Wilson,1984). One might argue that the injury which happens to occur during reperfusion is a natural part of the pathogenesis of ischemic injury. This is in part a matter of semantics. Injury occurring during reperfusion may not be necessarily injury induced by reperfusion. However, the fact that in many studies the injury that occurs in association to reperfusion has been shown to be sensitive to therapeutical treatment warrants it to be considered as an entity. As the use of the word reperfusion injury is likely to recall this discussion over and again, a better, non-polemic description, might be ischemia-reperfusion associated injury.

Ischemia and reperfusion can occur simultaneously in a single organ. Complete organ ischemia followed by sudden reperfusion is rather an exceptional situation which is mainly encountered in experimental models. In clinical myocardial infarction e.g., varying degrees of hypoxia and partial ischemic areas are more likely to occur. In the setting of organ transplantation, however, complete organ ischemia and reperfusion are distinct situations, clearly separated in time. Nonetheless, partial ischemia may also occur as a complication of donor nephrectomy and implantation.

\section{Reactive oxygen metabolites}

In recent years, ischemia-reperfusion associated injury has been generally attributed to the action of reactive oxygen metabolites (Hernandez,1987). The generation of free radicals following reoxygenation may lead to lipid peroxidation and increased membrane permeability. The enzyme xanthine oxidase has been supposed to play a main role in the generation of oxygen free radicals. The most, strong evidence for this assumption was the finding that allopurinol which is a well-know inhibitor of xanthine oxidase activity improves postischemic recovery to a simillar extent as radical scavengers (McCord,1984; Hearse, 1984). Xanthine oxidase arises in ischemic tissue by conversion of xanthine dehydrogenase through the action of a protease which is only active in ischemic conditions. The enzyme activity catalyzes the conversion of hypoxanthine to xanthine. Since there is a close relationship between $\mathrm{pO}_{2}$ and the activity of xanthine oxidase the formation of radicals is observed not until reperfusion following an increase of tissue $\mathrm{pO}_{2}$. The importance of this enzyme as a major source of free radical generation has recently been seriously doubted as it was shown that the activity of the enzyme is strongly tissue and species dependent (Southard,1987). Such a dependency has not been observed with respect to the occurrence of free radical injury, suggesting a minor role of xanthine oxidase in the generation of reactive oxygen metabolites.test In tissues which are known to have a considerable concentration of xanthine oxidase, e.g. the epithelium of the small bowel, this enzyme is still considered as a major source of oxygen radical formation (Hernandez,1987).

\section{Microvascular plugging}

In studies on myocardial infarction a persistent absence of flow despite the start of reperfusion has been observed. The mechanical obstruction of small capillaries by accumu- 
lating blood elements, especially white blood cells has been considered the main cause of this no-reflow phenomenon. The increased vascular resistance may lead to sustained ischemic damage. Similarly, severe tissue edema may evolve from subsequent increased capillary permeability (Engler,1986). Obstructive lesions have been reported in kidney experiments as well. In the outer zone of the medullar region trapping of blood elements during postischemic reperfusion has been observed. The effects of the obstruction is considered to play an important role in the development of postischemic acute renal failure (Mason,1987).

\section{Vasoactive substances}

A large number of circulating vasoactive substances have been observed following ischemia. The release of these substances has been regarded as an general attempt to maintain arterial blood pressure by increasing peripheral vascular resistance. Locally, the effect in the ischemically damaged organ may be adverse, further promoting ischemia. In studies on myocardial ischemia such effects have been related to the endogenous secretion of leukotrienes, tromboxanes, and platelet activating factor (Lefer,1987). In kidney, adverse effects on postischemic blood flow have been attributed to the action of adenosine (Oswald,1978).

\section{INFLAMMATORY RESPONSE}

The true nature of these classic mechanisms of reperfusion injury is still a matter of discussion. A common denominator linking these mechanisms in one single concept has not been established. Furthermore, in kidney the eventual and proportional effect of these processes is hard to establish. A remarkable capacity to recover from extensive parenchymal necrosis after a long period of time has been well established. Acute changes in tissue integrity and renal function are, therefore, not necessarily reflective of the final outcome of the recovery process (Fried,1984). Most of our knowledge on the damaging effect of mechanisms as described above is obtained from intervention studies in which a particular mechanism is eliminated (Hearse,1984). From such studies evidence is arising in support of the assumption that inflammation is an important and general determinant of ischemia-reperfusion associated injury. It may be surprising that the inflammatory response to ischemically damaged tissue has received relatively less attention whereas the general role of inflammatory processes in tissue injury has been well recognized. Perhaps, inflammatory processes are thought to be essentially involved in repair mechanisms and are not expected to have negative side effects (Henson,1987; Reimer, 1984). In studies on the use of pharmacological drugs to reduce infarct size it was observed that a remarkable improvement of recovery could be obtained without obvious changes to hemodynamic, metabolic and collateral blood flow related parameters. For instance, the beneficial effect of the prostacyclin analogue Iloprost appeared to be unrelated to tissue high energy phosphates, hemodynamic changes, or a free radical scavenging action towards xanthine oxidase activity in dog heart. In vitro studies, however, revealed a reduced production of oxygen reactive metabolites testby neutrophils following Iloprost treatment (Farber,1988). Similarly, the reduction of myocardial infarct size by $\mathrm{PGI}_{2}$ infusion has been attributed to inhibition of neutrophil activation (Simpson,1987). These data suggest that certain drugs exert a beneficial effect through their interaction with neutrophil mediated inflammatory reactions rather 
The role of neutrophils is generally recognized whether in classic mechanisms or as part of the inflammatory response. A key question seems to be whether the neutrophil involvement is a major cause of ischemia-reperfusion associated injury or is merely a result of this injury with only negative side effects like capillary plugging. Presently, there is much evidence that neutrophil accumulation may be considered both as an effect and a cause of ischemia-reperfusion associated injury. This option was recently highlighted as it appeared that the xanthine oxidase generated free radicals might induce neutrophil accumulation in the ischemic small bowel $($ Grisham,1986) whereas subsequent studies in a similar model revealed that specific inactivation of neutrophils considerably reduced free radical mediated tissue damage suggesting a clearly damaging role for the neutrophils (Hernandez,1987). Further evidence in support of this option is provided by recent experimental observations which suggest that the capillary obstruction by neutrophils and the inflammatory activity of these cells are independent phenomena. Early after the onset of myocardial infarction obstruction of true capillaries is observed whereas adherence of neutrophils to the endothelial lining of venules and arterioles as well as migration of these cells occurs only after some time (Engler,1986). It should be noted, however, that neutrophil involvement may not explain all of the ischemia-reperfusion associated injury. Tissue damage during reperfusion has also been observed in isolated organ perfusion systems using electrolyte solutions free of blood elements and thus the effects of neutrophils.

\section{Initiation phase}

For a proper discussion of the mechanisms that constitute the inflammatory response to ischemic tissue injury it may be helpful to distinguish inflammation initiating factors from mechanisms that are supposed to induce damage.

The first obvious sign of inflammation is the invasion of neutrophils in the infarcted area. Since the extent of neutrophil accumulation appears to be related to the extent of ischemic injury (Engler,1986) some kind of regulatory mechanism is assumed to be involved. Mechanical entrapment cannot be the only reason for the presence of the neutrophils. The reduced perfusion pressure in the ischemic area following myocardial infarction leads to capillary entrapment of neutrophils. However, reinstallment of tissue perfusion in the infarcted area induces an increase of the tissue content of neutrophils rather than a removal of the infiltrating cells (Engler,1986).

Data are currently available that strongly favour the release of tissue factors from. ischemic tissues that attract neutrophils. It is interesting to note in this respect that the infiltration of ischemic tissue by neutrophils is not necessarily dependent to reperfusion. Even when a kidney is completely dissected from its surroundings and stored in the peritoneal cavity accumulation of white cells can be observed within few hours (Sheehan, 1953). In agreement with this observation are findings that neutrophils accumulate in non-perfused areas of the myocardium during permanent infarction (Engler,1986). A variety of mediators can be considered. A potential role for the macrophage product GM-CSF (granulocyte-macrophage colony stimulating factor) has been suggested. Although the effect of this cytokine was assumed to be confined to hematopoietic progenitor cells recently evidence has been provided that GM-CSF is also involved in the 
activation of neutrophils. Effects on migration, oxygen radical formation, and cytotoxicity of neutrophils have been reported. Similarly, GM-CSF may stimulate destructive properties of macrophages. The origin of GM-CSF in vivo is not yet clearly defined. In addition to $T$ lymphocytes, fibroblasts, and macrophages, however, endothelial cells may also act as a sources of this cytokine (Thorens,1987).

A number of cells of the renal parenchyma are able to produce PAF (platelet activating factor). This phospholipid compound is known to have a number of local and systemic activities that are involved in inflammatory reactions. Locally, PAF affects vascular permeability and induces aggregation and activation of platelets as well as neutrophils. The effect on target cells is mediated by a PAF induced transient increase of intracellular calcium. Renal production of PAF has been observed following calcium inonophore induced membrane damage (Schlondorff,1986).

In addition, it has recently been postulated that the infiltration of neutrophils in ischemic tissue may directly evolve from the generation of free radicals by xanthine oxidase in the injured cells (Grisham,1986). An indirect way in which stimulating mediators may be released becomes plausible if one considers that invading neutrophils are killed by the ischemic conditions in the infarcted area (Sheehan,1953). Their death and lysis may again offer a stimulus for inflammatory reactions. Pertinent to this assumption are observations of ringlike accumulations of neutrophils that move outwards from the centre of the infarction by a steady invasion of the neutrophils in the border zone (Hearse,1984). The importance of the endothelium in mediating the activation of neutrophils has recently been convincingly demonstrated in vivo. The adhesiveness of neutrophils to endothelium and other subtrates is related to the membrane glycoprotein complex CD 18 (Harlan,1987). A monoclonal antibody directed against a function-related epitope on CD 18 has shown to be effective to prevent neutrophil accumulation in ischemic tissues (Hernandez,1987). The importance of this step is evident from studies showing an increase in survival after haemorrhagic shock by treatment with a monoclonal antibody against CD 18 in rabbits (Vedder,1988).

\section{Effector phase}

The damaging effect of locally accumulated neutrophils has previously considered to result from mechanical obstruction. There is much evidence now that activated neutrophils exert damage in a more direct way. A toxic effect on endothelial cell has been suggested to be due to the production of reactive oxygen metabolites by neutrophils (Chopra,1987). Evidence for the importance of this phenomenon in vivo has been recently presented in a study on small bowel ischemia (Hernandez,1987). Damage to the glomerular basement membrane and the disturbance of glomerular metabolic processes can also be induced by activated neutrophils. The cellular content of cyclic AMP is increased through the action of the neutrophils which may lead to a reduction of the glomerular ultrafiltration coefficient (Basci,1987). Although the extravascular migration of neutrophils has been recognized, the endothelium and the capillary lumen is usually regarded as the site of action. Neutrophil mechanisms that might elicit damage to parenchymal cells have not yet been established in this respect.

Secondary to neutrophil invasion, migration of macrophages in inflammatory tissues has been observed. Macrophages are known to play an essential role in the effector phase 
of the specific immune response as well as in non-specific inflammatory reactions. The effector mechanisms of the macrophages are closely associated to the cyclooxygenase and lipoxygenase pathways of the arachidonate metabolism. Inhibitors of these pathways interfere with the production of a large number macrophage products. Among these products are beta-glucuronidase, $\mathrm{PGE}_{2}$, thromboxane $\mathrm{B} 2$, platelet activating factor, each with potent effects on cell function and integrity.

In addition to the effect of generally released substances, mechanisms are known in which cells are individually killed. Cytotoxic T lymphocytes have been shown to kill their target cell by the release of a pore-forming protein after making close contact to the membrane of the target cell (Young,1987). Similar mechanisms may be found in cells that are activated during an inflammatory reaction. A close cell to cell contact has been observed in killing by macrophages. A number of lytic factors may be involved in this contact, including esterases, reactive oxygen metabolites, and TNF (tumor necrosis factor).

The latter is a cytokine produced by macrophages with various effects both at a cellular and systemic level. TNF has been measured in plasma of patients with fulminant inflammatory processes (Beutler,1987). A number of other cytokines have properties which make them also likely to be involved in the effector phase of an inflammatory reaction. Their rolle in ischemia-reperfusion associated injury has, however, yet to be established. Cytotoxicity of cytokines has generally been studied with respect to inflammatory cells. Recent data, however, suggest that cytokines may have also toxic effects on normal parenchymal cells. In vitro studies on tubular cells from human kidneys showed that treatment with a combination of interleukin 1 and gamma interferon induces a severe loss of functional and structural integrity of the renal cells (chapter 14).

PAF, which may be produced by non-tubular renal cells as well as by inflammatory cells, can disturb renal function by changing intrarenal blood flow distribution, and by inhibition of glomerular filtration rate and sodium excretion (Schlondorff,1986). Since this substance can simultaneously activate platelets and neutrophils, PAF may appear to play a pivotal role in the mediation of the inflammatory response.

\section{MODULATION OF THE INFLAMMATORY RESPONSE}

One approach to investigate the role of the inflammatory response in the development of ischemia-reperfusion associated injury is to stimulate rather than to suppress the activity of inflammatory mechanisms. Enhancement of the inflammatory reactivity may reveal insight in the potential destructive capacity of the inflammatory response in the ischemic tissue. This, at a first glance artificial thought has an important clinical counterpart. The development of acute renal failure with severe cortical ischemia is a notorious complication in patients with overwhelming inflammatory disease as a result of a gram negative bacterial infection. Although the pathogenesis of this renal damage is not yet completely understood there is good evidence that the cortical ischemia may only in part result from hemodynamic instability. The exacerbation of the ischemic damage has been attributed to a number of secondary effects of the septicemia.

Recently, experimental data have been obtained that are in support of this hypothesis. The effect of a moderate ischemic renal insult in rat is remarkably extended when the animals are simultaneously injected with Escherichia coli endotoxin. The extent of the ischemic damage is increased as appears from a delay in postischemic recovery and his- 
tological examinations. If kidneys are not previously rendered ischemic the endotoxin injections have no effect, neither on renal function and histology nor systemically (Zager,1986; chapter 15). These data may suggest that if the inflammatory response which is elicited by the ischemic tissue injury is enhanced by a second stimulus, the negative effects of the inflammatory response are potentiated.

\section{Endotoxin}

Endotoxin is a general stimulus of inflammatory processes. A wide range of effects of endotoxin on various inflammatory mechanisms have been described (Morrison,1978) and are still being discovered. Large amounts of circulating GM-CSF in the blood are induced by systemic injections of LPS (Thorens, 1987). The cytolytic capacity of macrophages is increased in response to LPS in the presence of a second mediator (recombinant gamma interferon or other lymphokines) (Mauel,1987). Additional effects of endotoxin have been reported on the function of target cells. Generation of reactive oxygen metabolites within endothelial cells together with a stimulation of antioxidant enzyme activity has been suggested from in vitro studies with LPS stimulation (Shiki;1987). Others found a decrease in activity of cellular cyclic AMP in parenchymal cells which lead to a reduced hormonal responsiveness following LPS treatment (Shepherd, 1987). Such mechanisms may increase both the destructive capacity of the inflammatory reactions and the sensitivity of the tissues that are involved.

\section{Shwartzman-like reactions}

There is however a more specific reaction of the inflammatory system that has been associated with endotoxin. As early as in 1928 it was observed that a second intracutaneous injection of bacterial endotoxins 24 hours after a first injection elicits a fulminant local inflammatory response leading to necrosis (Shwartzman,1928). In later studies this Shwartzman reaction as it is called, also appeared to involve general organ systems if the endotoxin injections are given intravenously (Good,1952). Characteristically, two signals are necessary to elicit a Shwartzman reaction. In addition, the time interval between the administration of the two stimuli markedly influences the eventual effect. Recently, this phenomenon was further analyzed at the background of newly discovered inflammatory mechanisms. A number of cytokines including TNF, interleukin 1, and gamma interferon, have been shown to be able to replace part of the original endotoxin stimuli (Billiau,1988). Similarly, stimulation with immune complexes or zymosan in combination to exposure to endotoxin induces a fulminant inflammatory reaction (Movat,1987). It is tempting to speculate that the two signal concept is involved in the interaction of inflammatory mechanisms and ischemic tissue injury. In this view the latter might be responsible for one of the two stimuli.

\section{Potentiation}

For some unknown reason a time interval of 18 to 24 hours between the first and second stimulus appears to be crucial in the induction of a Shwartzman reaction. The explanation may be trivial, and related to kinetic properties of the mediators involved. If not, the importance of the Shwartzman reaction to native, in vivo occurring processes may remain questionable. Recently, however, similar synergistic reactions in response to endotoxin and a second stimulus have been reported in which both stimuli were given 
simultaneously. Treatment of mice with the hepatotoxic agent D-galactosamine together with endotoxin induced a remarkable increase of lethality. This increase could not be explained from summation of the effects of treatment with the individual compounds. Interestingly, the endotoxin stimulus could be replaced by TNF suggesting an important role for macrophages (Lehmann,1987). Other synergistic actions with endotoxin in combination to cytokines are known to lead to macrophage activation (Mauel,1987).

\section{Tumor necrosis factor}

The working mechanism of endotoxin has recently been closely associated with the production and effects of TNF. LPS stimulates macrophages to the production of TNF in vitro as well as in vivo (Old,1987). The effects that are found after LPS injection are largely similar to the effects that are obtained by systemic TNF treatment (Kettelhut,1987).

Studies in rat in which ischemic renal injury was potentiated by LPS stimulation were recently repeated using TNF instead of LPS. A similar worsening of renal function and delay in recovery was observed. In some cases the combination of TNF treatment and moderate renal ischemia appeared to be lethal within 24 hours. Treatment with TNF alone did not affect renal function nor induced mortality (chapter 15).

\section{FUTURE PROSPECTS}

In this chapter a number of mechanism have been discussed that may evolve from an inflammatory response to ischemically damaged tissues. The actual impact of these mechanisms on ischemia-reperfusion associated injury has yet to be determined. Concomitant to our growing understanding of inflammatory mechanisms there is currently evidence arising that this impact is more extensive than was originally expected.

The involvement of an inflammatory response to the eventual outcome of postischemic renal recovery is clearly a complicating factor to viability assessment. The time course of events that result from inflammation is unknown but certainly exceeds the period in which common viability tests are performed. In general, however, the effect of ischemiareperfusion associated injury will depend on the extent of the original ischemic impetus and is one factor that determines what is commonly called, ischemia tolerance. The predictive value of most viability tests is largely based on the empirically obtained notion of renal ischemia tolerance. It is unfortunately only in this way that the role of ischemiareperfusion associated injury is presently considered in viability assessment.

A better understanding of the inflammatory response following ischemic injury may, however, lead to the development of diagnostic tools that can improve the monitoring of ischemic injury. It has previously been suggested that there is a obvious relationship between the extent of ischemic injury and the extent of the inflammatory response. Neutrophils are specifically attracted to and activated in the infarcted area. If this procedure appears to be receptor-mediated as is plausible from a general point of view on immune system associated working mechanisms, an imaging technique using labeled antibodies against these receptors may be developed to monitor the extent of the infarction. Although the application of such a technique is still highly speculative promising results have already been obtained in a distantly related field using radio labelled small diameter colloids to image inflammatory lesions (Chia,1986). Furthermore, activating mediators or reactive oxygen metabolites released from ischemically injured cells during reperfu- 
sion may become measurable and indicative for the extent of injury.

A second important prospect of a better understanding of postischemic events is a rational approach to the improvement of therapeutic interventions. The treatment of ischemic injury may appear particularly suitable in controlled situations like organ transplantation. In the last few years new therapeutic strategies for the intervention in inflammatory reactions are studied which may have important consequences for future treatment of ischemia-reperfusion associated injury. PAF antagonists have been successfully used to inhibit the effects of PAF in isolated kidney perfusions (Schlondorff,1986). Indomethacin is a well-known effective blocker of the cyclo-oxygenase activation pathway in macrophages. Recent in vivo studies showed that indomethacin can considerably reduce mortality in TNF-induced shock (Kettelhut,1987). Another approach to interfere with TNF mediated injury is represented by the use of monoclonal antibodies directed against TNF. From in vitro studies it is known that monocional antibodies may completely inhibit the specific action of TNF. Recently, it was demonstrated that monoclonal anti TNF therapy can be successfully applied in vivo. Prophylaxis with anti TNF antibodies prevented dying of animals with severe Escherichia coli induced sepsis (Tracey,1987). These observations implicate that TNF is a pivotal mediator of profound inflammatory reactions. Furthermore, it is suggested that a complex and overwhelming inflammatory reaction in which numerous mediators and cellular mechanisms are known to be involved can be influenced by inhibiting the action of a single mediator. 


\section{CHAPTER 4}

\section{DETERMINATION OF WARM ISCHEMIA TIME DURING DONOR NEPHRECTOMY ${ }^{1}$}

\section{INTRODUCTION}

In order to meet the increasing demand for donor kidneys, criteria for donor suitability have been relaxed. Kidneys from non-heartbeating donors and donors with prolonged severe hypotension are currently considered for transplantation (Kootstra,1986). Obviously a clinical test to evaluate the suitability of these kidneys before they are transplanted is required but is not yet available. Many factors affect the eventual life-sustaining function of a graft. Warm ischemia time has been considered as the most important factor and it is determined from the time interval between cessation of circulation, respectively the excision of the organ and the start of the hypothermic flush procedure. Before the start of the warm ischemia time additional ischemic damage may be evoked by hemodynamic instability of donor origin or surgical manipulation during dissection (Horpacsy, 1980). This additional ischemic damage is not easily assessed and generally remains unnoticed.

Many attempts have been made to estimate the actual ischemia time by monitoring the catabolism of high energy compounds. It has been reported that determination of ATP levels is not useful in this respect as the ATP content of renal tissue is already virtually depleted within few minutes after the onset of ischemia (Bore,1979). In contrast, total adenine nucleotide levels, i.e. the sum of ATP, ADP, and AMP, have been suggested to be better related to the duration of ischemia (Calman,1974). Whether this relationship can be used to develop a clinical test to monitor ischemia is incompletely explored. Furthermore monitoring of IMP, nucleosides (Ino, Ado), and oxypurines ( $\mathrm{HX}, \mathrm{X}$ ) might offer a more adequate method to determine the level of break down of the adenine nucleotides as these degradation products accumulate in the ischemic tissue (Buhl,1979; Takenaka,1980).

The present study was designed to investigate the correlation between ischemia time and the ratio of the adenine nucleotides and their degradation products in cortical tissue of canine kidneys. Recent developments in HPLC technology allow determination of these metabolites in small tissue samples (Wynants,1985). The suitability of cortical wedge biopsies to deliver tissue specimen for this HPLC technique was tested since this type of biopsies can be obtained in a clinical setting.

Levels of purine metabolites were determined at ischemic intervals ranging from 0 to 120 minutes. As the temperature might affect the influence of ischemia on the energy metabolism, ischemia was induced both at $37^{\circ} \mathrm{C}$ and at $32^{\circ} \mathrm{C}$, assuming that during donor nephrectomy the temperature stays within this range. In addition to animal experiments, the suitability of human wedge biopsies obtained from non-ischemic donor kidneys, was 
tested for analysis of purine metabolism. We found that the ratio of the levels of adenine nucleotides and their degradation products presents a sensitive index of preceding ischemia time. It appeared that a relatively small decrease of temperature, reduces the rate of break down of the adenine nucleotides.Moreover, it is shown that small wedge biopsies of human kidneys can be used to determine the concentration of purine metabolites.

\section{MATERIALS AND METHODS}

\section{Animals}

Experiments were performed on adult mongrel dogs of both sexes weighing between 18 and $25 \mathrm{~kg}$. Anesthesia was induced with thiopentotal ( $20 \mathrm{mg}$ per $\mathrm{kg}$ body weight) and maintained with a nitrous oxide-oxygen gas mixture, delivered continuously by a positive pressure respirator wia an endotracheal tube. The animals were placed on a thermostatic watermattress. Both kidneys were exposed through a midline abdominal incision.

\section{Biopsy technique}

Biopsies were harvested with two different techniques. A first series of biopsies was obtained with a dentist drill (group $A, N=6$ ). The tissue samples were submerged in liquid nitrogen within 3 seconds. In a second series cortical wedge biopsies were taken with a surgical blade (group $\mathrm{B}, \mathrm{N}=6$ ). With this technique it took $15-20$ seconds between the incision and freezing of the samples in liquid nitrogen.

\section{Experimental protocol}

Subsequently the effect of ischemia on the tissue content of adenine nucleotides (ATP, ADP, and AMP) and their degradation products (IMP, Ino, Ado, HX and X) was assessed at $37^{\circ} \mathrm{C}$ (group C) and $32^{\circ} \mathrm{C}$ (group D). The wedge biopsy technique was used in both groups. In group $\mathrm{C}(\mathrm{N}=6)$ the right kidney was carefully dissected from its surrounding tissue and a clamp was placed on the vessel pedicle and ureter. Cortical wedge biopsies were taken at $0,30,60,90$, and 120 minutes after the onset of ischemia. In group $\mathrm{D}(\mathrm{N}=7)$ the right kidney was excised and stored in an incubator at $32^{\circ} \mathrm{C}$. Cortical wedge biopsies were taken at the same intervals as in group $\mathrm{C}$.

\section{Human biopsies}

The concentration of purine metabolites was determined in tissue specimens from human donor kidneys $(\mathrm{N}=12)$. Small cortical wedge biopsies were taken at donor nephrectomy before blood circulation through the kidney was interrupted and before cooling was started. The excision time was recorded. All samples were frozen in liquid nitrogen.

\section{Analysis of metabolites}

All cortical tissue samples were stored at $-80^{\circ} \mathrm{C}$. Before analysis the tissue samples were lyophilized at $-30^{\circ} \mathrm{C}$. Adherent blood was removed. The samples were weighed and extracted in $25-50 \mu \mathrm{l} / \mathrm{mg}$ tissue $\mathrm{HCLO}_{4}(2 \mathrm{~mol} / \mathrm{h})$. The tissue $\mathrm{HCLO}_{4}$ mixture was centrifuged at $1200 \mathrm{~g}$ for 5 minutes at $4^{\circ} \mathrm{C}$ and the supernatant was frozen in liquid nitrogen. Following addition of $40-80 \mu / / \mathrm{mg}$ tissue $\mathrm{KHCO}_{3}(2 \mathrm{~mol} / \mathrm{l})$ to the supernatant, this mixture was allowed to thaw during centrifugation at $1200 \mathrm{~g}$ at $4^{\circ} \mathrm{C}$ for 1 hour, during which 
neutralization took place.

Analysis of the samples was performed by a slightly modified version of the HPLC technique described by Wynants and van Belle (1985). A Varian vista 5000 (Varian, Wallnut Creek, California) equipped with a narrow bore, stainless steel column filled with spherical lichrosorb RP-18 particles of $5 \mu \mathrm{m}$ (Merck,Darmstadt,FRG) was used for analysis. A gradient system was applied. Solvent $\mathrm{A}$ contained $0.15 \mathrm{M} \mathrm{NH}_{4} \mathrm{H}_{2} \mathrm{PO}_{4}$ in water buffered to $\mathrm{pH} 6.0$ with $\mathrm{NH}_{4} \mathrm{OH}$. A mixture of acetonitrile and methanol $(1 / 1$, by volume) was used as solvent $B$. The first seven minutes were kept isocratic with solvent $A$. Thereafter a gradient was installed from $100 \%$ solvent A to $85 \%$ solvent A during 23 minutes. The flow rate was linearly increased from 0.7 to $0.8 \mathrm{ml} / \mathrm{min}$ within the first 3 minutes and kept at $0.8 \mathrm{ml} / \mathrm{min}$ during the remaining 27 minutes of the run. After each single run the column was re-equilibrated during 15 minutes with $100 \%$ solvent $\mathrm{A}$. All chemicals were obtained from Merck. Peaks were quantitated at $254 \mathrm{~nm}$ with a Varian 604 datasystem using the relationship of peak area per concentration of a known external standard. Standards were of the highest purity available. ADP, AMP, and IMP were purchased from Boerhinger (Mannheim,FRG), HX and Ado from Merck and ATP, Ino, and X from Sigma. This technique offered effective separation of the nucleotides, nu-

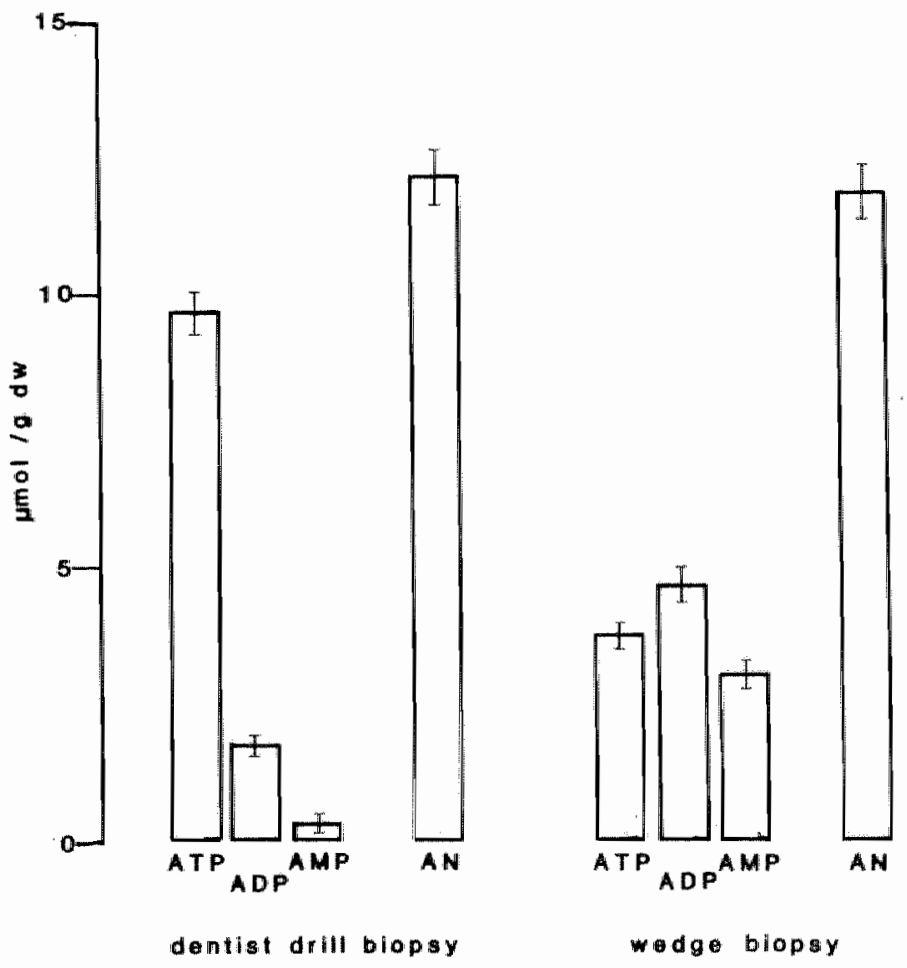

Figure 4.1.: The content of adenine nucleotides in normoxic renal cortical tissue. Tissue specimen were obfained with the dentist drill or the wedge biopsy technique in dogs. Time delay between incision of the renal cortex and freezing of the tissue specimen amounted to 1-3 and 15-30 seconds, respectively. Data refer to mean $\pm \operatorname{SEM}(\mathrm{N}=6$ in both groups) and are expressed as $\mu \mathrm{mol} / \mathrm{g}$ dry weight of tissue. 
cleosides, and oxypurines, assessed in one single run.

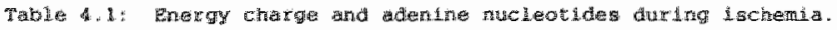

\begin{tabular}{|c|c|c|c|c|c|c|c|c|c|c|}
\hline \multicolumn{2}{|c|}{$\begin{array}{l}\text { Wenethita } \\
\text { tume }\end{array}$} & \multicolumn{2}{|c|}{ ATY } & \multicolumn{2}{|c|}{$\mathrm{ADP}$} & \multicolumn{2}{|c|}{ AMP } & $\begin{array}{l}\text { Rargy } \\
\text { Ctuarge }\end{array}$ & \multicolumn{2}{|c|}{ TAHC } \\
\hline controu & & 4.34 & \pm 0.44 & 4.71 & \pm 0.29 & 2.80 & 20.16 & $0.56 \pm 0.02$ & $12.85=$ & \\
\hline \multirow[t]{2}{*}{$30 \mathrm{~m}: \mathrm{Ln}$} & $3 x^{6} \mathrm{C}^{2 \mathrm{ai}}$ & 0.73 & +0.07 & A. 31 & $\$ 0.0 .5$ & 9.05 & $\div 0.45$ & $0.20 \neq 0.011$ & 7.09 & 20.5 \\
\hline & $32^{0} c_{w}$ & 0.5 .1 & +0.08 & 1.38 & \pm 0.04 & 7.89 & $: 0.40$ & $0.14 \pm 0.01$ & $9.80 *$ & $\leqslant 0.3$ \\
\hline \multirow[t]{2}{*}{$60 \mathrm{~min}$} & $370 \mathrm{C}$ & 0.49 & \pm 0.02 & 0.95 & \pm 0.07 & 3.37 & \pm 0.33 & $0.20 \pm 0.01$ & $4.80=$ & \pm 0.3 \\
\hline & $320^{C} \mathrm{C}$ & 0.90 & \pm 0.110 & 1.30 & \pm 0.04 & 6.70 & 9.60 & $0.14=0.022$ & 9.40 & $=0.4$ \\
\hline \multirow[t]{2}{*}{$30 \mathrm{~m} \cdot \mathrm{Ln}$} & $370 \mathrm{C}$ & 0.46 & $\$ 0.07$ & 0.91 & \pm 0.05 & 2.92 & $=0.28$ & $0.25 \pm 0.02$ & . $29+$ & $* 0.3$ \\
\hline & $320 \mathrm{C}$ & 0.32 & \pm 0.04 & 1.14 & \pm 0.03 & 6.20 & \pm 0.10 & $0.12=0.011$ & 7.60 & \pm 0.1 \\
\hline \multirow[t]{2}{*}{$120 \mathrm{~m} n \mathrm{n}$} & $37^{\circ} \mathrm{C}$ & 0.44 & 1. 0.04 & 0.61 & \pm 0.04 & 2.157 & $=0.27$ & $0.23 \pm 0.022$ & $3_{n} 22$ & +0.2 \\
\hline & $32^{0} \mathrm{C}$ & 0.33 & 0.09 & 1.11 & \pm 0.07 & 5.30 & \pm 0.20 & $0.13=0.01$ & 6.80 & \pm 0.3 \\
\hline
\end{tabular}

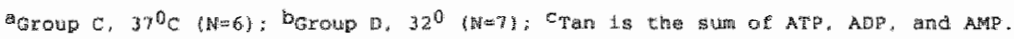

\section{Data analysis}

All values reported are given as mean \pm standard error. The concentrations of the purine metabolites are expressed as micromoles per gram tissue dry weight. They were compared at the various ischemic intervals by Wilcoxon rank-sum test. Significance was assessed by a $\mathrm{p}<0.05$. The effect of temperature as well as harvest time of the biopsies

Table a. 2: Accumitation of degradation products during ischenta.

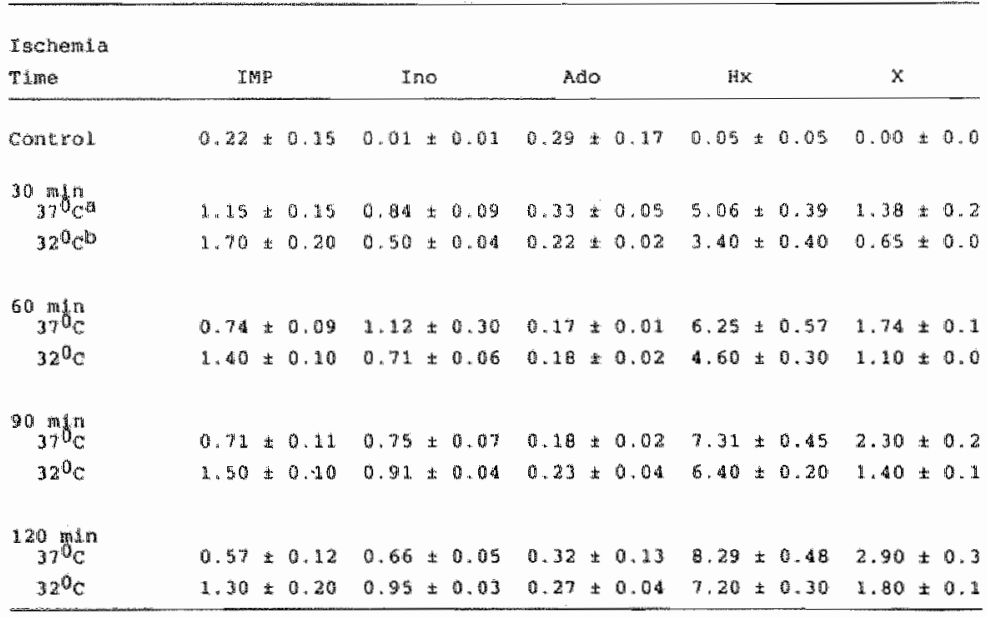

aroup $C, 390^{\circ},(N=6): b$ Group $D, 32^{\circ} \mathrm{C} \quad(N=7)$. 
on the catabolism of the metabolites was tested by the Mann-Whitney U test. Significance for this test was assessed by a $p<0.05$. The energy charge was calculated as (ATP $+1 / 2 \mathrm{ADP}) /(\mathrm{ATP}+\mathrm{ADP}+\mathrm{AMP})(\mathrm{Atkinson}, 1968)$.

\section{RESULTS}

Biopsy technique

Normoxic levels of adenine nucleotides (ATP, ADP, and AMP) in cortical biopsies obtained with two different techniques are shown in figare 4.1. In biopsies harvested with
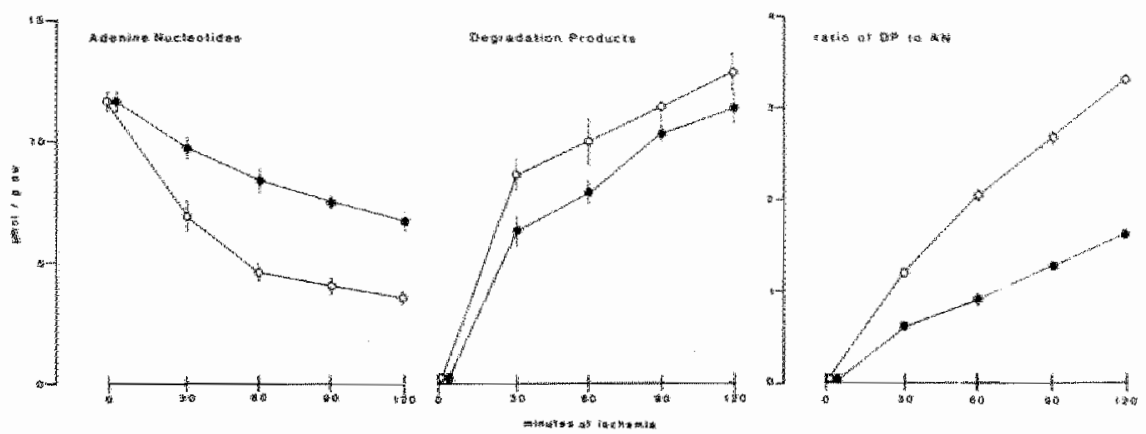

Figure 4.2.: Time course changes in the content of adenine nucleotides and degradation products in ischemic renal cortical tissue at $37^{\circ} \mathrm{C}$, and $32^{\circ} \mathrm{C}, 2 \mathrm{~A}$ : Decrease of the content of the sum of adenine nucleotides i.e. ATP, ADP, and AMP (AN). 2B: Increase of the content of the sum of degradation products i.e. IMP, Ino, $A d o, 1 x$ and $X(D P) .2 \mathrm{C}$. Ratio of the content of DP to AN in renal cortical tissue as function of the duration of ischemia. Data refer to mean $\pm \mathrm{SEM}\left(\mathrm{N}=6\right.$ in group $\mathrm{C}$ (open circle) $37^{0} \mathrm{C} \cdot \mathrm{N}=7$ in group $\mathrm{D}$ (closed eircie, $32^{\circ} \mathrm{C}$. Canine tissue specimen were obtained by the wedge biopsy technique.

the dentist drill and frozen within 3 seconds in liquid nitrogen (group A), ATP values accounted for about $80 \%(9.80 \pm 0.67 \mu \mathrm{mol} / \mathrm{g}$ dry weight) of the sum of the adenine nucleotides. In contrast ATP values were less than $30 \%(3.92 \pm 0.27 \mu \mathrm{mol} / \mathrm{mg}$ dry weight) of the sum in the wedge biopsies, frozen within $15-20$ seconds after the first incision (group B). The energy charge was $0.88 \pm 0.01$ and $0.53 \pm 0.02$ in group $A$ and group $B$, respectively. The prolonged harvest time with the wedge biopsy technique did not affect the sum of the adenine nucleotide content as shown in figure 1. In both groups the amount of degradation products was less than $10 \%$ of adenine nucleotide content. Ischemia: adenine nucleotides The effect of the time duration of ischemia on the catabolism of adenine nucleotides was studied at $37^{\circ} \mathrm{C}$ and $32^{\circ} \mathrm{C}$. In group C and D ATP levels were dramatically reduced after 30 minutes (table 1). Throughout the ischemic period no consistently significant effects of low temperature on the ischemia induced decrease of ATP could be observed. In contrast the differences of ADP and AMP levels at both temperatures became more pronounced during prolonged ischemia time. After the initial loss during the first 30 minutes, the decrease of ADP and AMP was significantly less in the low temperature group $(p<0.05)$. The energy charge was significantly reduced in both groups during the initial 30 minutes of ischemia $(p<0.05$ as compared with normoxic le- 


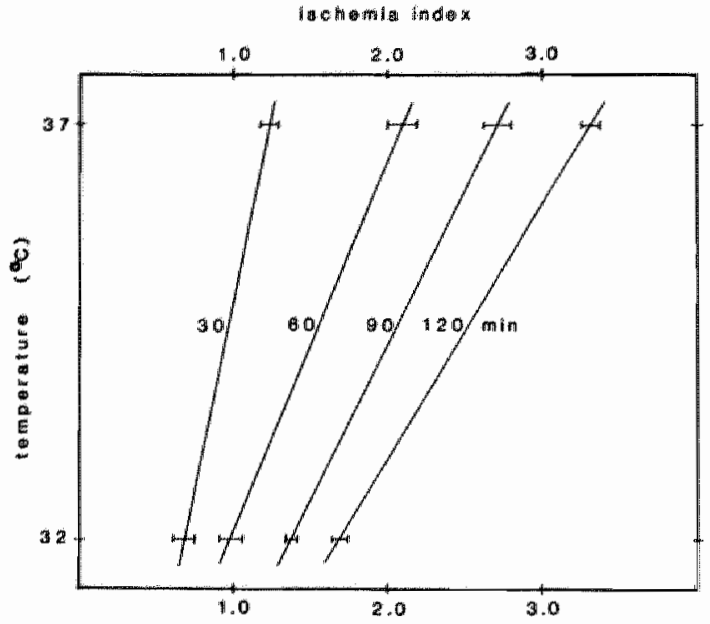

Figure 4.3.: Relation of ischemia index and the time duration of ischemia at $37^{\circ} \mathrm{C}$ and $32^{\circ} \mathrm{C}$. Purine catabolic index is defined as the ratio of degradation products (DP) to adenine nucleotides (AN). Mean values $\pm S E M$ are shown. Canine tissue specimen were obtained by the wedge biopsy technique.

vels), and remained at the same low level during the subsequent intervals. As a consequence of the substantially higher levels of AMP in the $32^{\circ} \mathrm{C}$ biopsies, the energy charge was significantly lower in this group than in the normothermic group at each time interval $(\mathrm{p}<0.05)$ (table 1$)$.

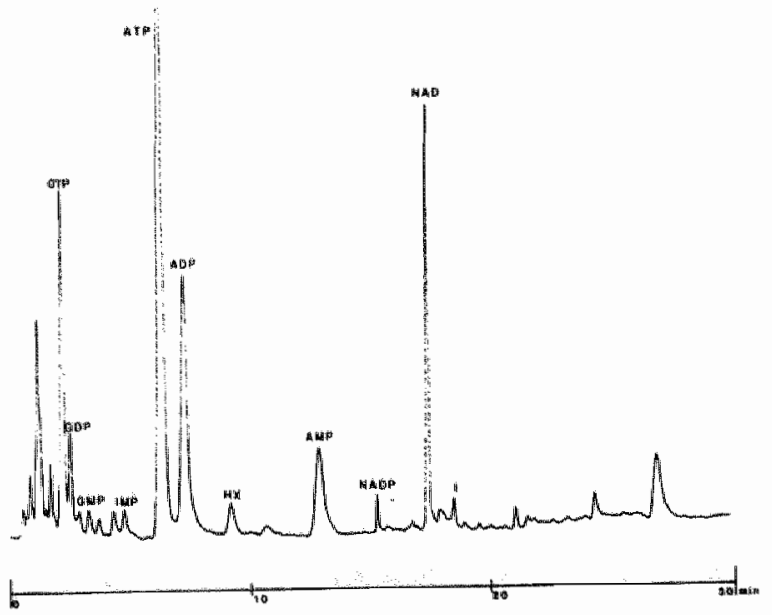

Figure 4.4: Typical chromatogram of extract of normoxic human renal cortical biopsy. Weight of the biopsy was $3.5 \mathrm{mg}$ dry weigth of tissue. $10 \mu$ from $250 \mu$ extract was injected on narrow bore, stainless steel column filled with sperical lichrosorb RP-18 particles of $5 \mu \mathrm{m}$. 


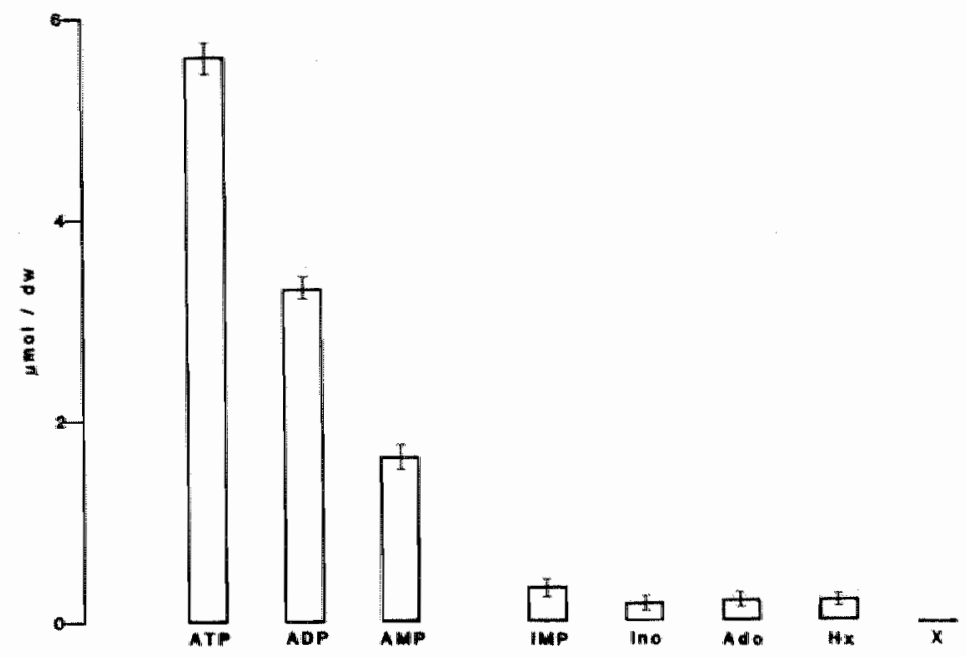

Figure 4.5.: Content of adenine nucleotides and degradation products in normoxic human renal cortical biopsies. Tissue specimen were obtained during donor nephrectomy with the wedge biopsy technique. Data refer to mean $\pm \operatorname{SEM}(\mathrm{N}=12)$ and are expressed as $\mu$ mol/g dry weight of tissue.

Ischemia: degradation products

The assessment of IMP, Ino, Ado, HX, and X in normoxic control tissue samples, obtained with the wedge biopsy technique (group $C$ and $D$ ), shows that the sum of these compounds is about $10 \%$ of total content of purine metabolites (table 2). The concentration of xanthine was below the level of detection. IMP levels showed an increase during the first 30 minutes of ischemia and returned to lower levels during the second thirty minutes but were still above control values (table 2). At each interval the values from the hypothermic group were significantly higher in comparison to the values of the body tem perature group $(p<0.05)$. Normoxic adenosine values remained only slightly above the level of detection and did not significantly change during ischemia. Inosine levels were also low. A small significant increase was found in both groups during ischemia $(\mathrm{p}<0.05)$. At body temperature the inosine levels tended to decrease from 60 minutes onwards but these changes did not reach the level of significance. Both hypoxanthine and xanthine increased with ischemia time in the normothermic group and to a lesser extent in the $32^{\circ} \mathrm{C}$ group. At the end of the 120 minutes ischemia interval more than $50 \%$ of the purine pool consisted of hypoxanthine in both groups. Thus it was found that degradation of adenine nucleotides causes accumulation of mainly hypoxanthine and to a lesser extent of xanthine. A decrease of the temperature affected the rate of degradation but did not substantially alter the pattern of the accumulated degradation products.

\section{Calculated data}

The sum of all purine metabolites (ATP, ADP, AMP, IMP, Ino, Ado, $H x_{\text {, }}$ and X) increased from $13.45 \pm 1.05 \mu \mathrm{mol} / \mathrm{g}$ dry weight (normoxic value) to $15.70 \pm 1.02$ in group 
$C$ and to $16.11 \pm 0.68$ in group $D$ during the first thirty minutes of ischemia. Following the first interval a continuous, moderate rise was observed to $16.55 \pm 0.99$ in group C and $18.26 \pm 0.77 \mu \mathrm{mol} / \mathrm{g}$ dry weight in group $\mathrm{D}$ at the end of the 120 minutes interval.

Further calculations revealed that the sum of the adenine nucleotides decreased and the sum of all degradation products increased with duration of ischemia time. The extent of these changes was affected by the temperature of the ischemic tissue (figure 4.2). The point at which the amount of degradation products exceeded the amount of adenine nucleotides, was delayed by reduction of the temperature to $32^{\circ} \mathrm{C}$.

Figure 4.3 shows that the ratio of adenine nucleotides and their degradation products is strongly correlated with ischemia time $(\mathrm{p}<0.05)$. Furthermore a significant difference $(p<0.05)$ was found between the values of the $37^{\circ} \mathrm{C}$ and $32^{\circ} \mathrm{C}$ group. Using the diagram that was constructed with these values (figure 3 ) the ischemia time of a tissue sample can be estimated by calculating the ratio of degradation products to adenine nucleotides. For example if this ratio is found to be 0.20 the corresponding ischemic interval is less than 30 minutes at any temperature between $32^{\circ} \mathrm{C}$ and $37^{\circ} \mathrm{C}$. A ratio of 3.30 or more indicates an ischemia time of at least 60 minutes. In this way a sensitive index is provided to asses the time duration of ischemia in renal tissue by analysis of purine metabolism in cortical wedge biopsies.

\section{Human biopsies}

Renal biopsies in this group were obtained during donor nephrectomy from donors which did not show severe hemodynamic disturbances before or during nephrectomy.The weight of the biopsies ranged from 8 to $35 \mathrm{mg}$ wet weight (mean value 18.95 $\mathrm{mg}$ ). The time needed to harvest the wedge biopsies until cooling ranged from 15 to 30 seconds. The profile of the purine metabolites is shown in figure 4.4 ATP levels were less than $10 \pm \mu \mathrm{mol} / \mathrm{g}$ dry weight in all biopsies (figure 5). The energy charge was high in comparison to the values from the corresponding canine biopsies $(0.68 \pm 0.03$ versus $0.53 \pm 0.02$, respectively).

The sum of the adenine nucleotides and the degradation products amounted to $10.63 \pm$ 0.34 and $1.20 \pm 0.19 \mu \mathrm{mol} / \mathrm{g}$ dry weight, respectively. The total content of purine metabolites was $11.84 \pm 0.29 \mu \mathrm{mol} / \mathrm{g}$ dry weight.

\section{DISCUSSION}

In order to develop an index of warm ischemia time for kidneys with obscure donor history, changes in concentrations of purine metabolites were measured during ischemia in biopsies obtained from canine renal tissue. The characteristic catabolic profile of the purine metabolites suggests a close relationship with ischemia time (Gerlach,1963). In previous studies adenine nucleotide metabolism was directly correlated to renal viability, i.e. recovery to life-sustaining function, with generally disappointing and conflicting results (Bergstrom, 1969; Pegg, 1984; Sells,1977). On the one hand, despite normalization of the energy status of the cells, functional recovery of the organ may be delayed by a variety of factors unrelated to the cellular energy metabolism. On the other hand, depletion of energy metabolites renders the kidney more susceptible to additional damage. The status of the cellular energy metabolism may therefore only correspond to organ viability when ischemia is the most important determinant of viability. Thus assessment of warm ischemia time should be regarded as a parameter of safety margins of 
viability rather than as a straight predictor of viability.

Our findings on the adenine nucleotide levels during ischemia, confirm the observations of previous studies that levels of these substances do not correlate consistently with the time duration of ischemia (Bore,1979; Isselhard,1972). Changes of the ATP content and the energy charge were found to be insignificant after 30 minutes of ischemia and the levels of ADP and AMP show a rise as well as a fall during the time course of the catabolic event. When the sum of these metabolites is taken into consideration, a more straightforward relation to ischemia time is found (Fischer,1981). In addition, the extent of nucleotide degradation may be interpreted more accurately when the concentration of the degradation products is considered as well. The present study indicates that the balance of adenine nucleotides and degradation products correlated strongly to ischemia time. Thus, a sensitive index of ischemia is provided, based on the above mentioned ratio to enable the assessment of warm ischemia time of donor kidneys during nephrectomy. The most prominent and rapid effect of ischemia on the energy metabolism of renal cortical cells is depletion of ATP starting within seconds after cessation of oxygen supply. To determine control values, methods to harvest tissue samples are therefore needed that will block the catabolic reaction as fast as possible. In this study tissue samples were obtained either with a dentist drill or with a surgical knife and submerged immediately in liquid nitrogen. Freeze clamping of whole organs is frequently used in other studies but this technique is only applicable to organs of small animals. The dentist drill technique offers a comparable harvest time as freeze clamping ( $1-3$ seconds). The latter technique was used to obtain control values for comparison with values reported in other studies and with values from the wedge biopsies. When biopsies are taken with a surgical knife, harvesting time is prolonged to $15-20$ seconds. Control biopsies showed that this additional ischemia time is reflected by lower tissue levels of ATP. The total adenine nucleotide content, however, remained unchanged. Moreover it appeared that the influence of the prolonged harvest time was no longer relevant at ischemic intervals of 30 minutes or more. Therefore with clinical application in mind we preferred the use of biopsies taken with a surgical knife in our experiments because the cylinder shape of the dentist drill increases the risk of injury to the intrarenal vessels of donor kidneys.

The determination of the energy status in cortical biopsies is not completely representative to the energy status of the whole organ. The effect of ischemia to the energy metabolism is different in the medulla compared to the cortex which may correspond to differences in ischemia susceptibility (Urbaitis,1984). The degradation of adenine nucleotides described in this study refers only to cortical tissue and will therefore largely reflect the break down in tubular cells.

Data on determination of the whole spectrum of purine metabolites in canine kidneys are scarce especially those including degradation products of adenine nucleotides during ischemia. Data on the adenine nucleotide content of non-ischemic tissue samples in rat, rabbit, and dog presented per mg dry weight and assayed either enzymatically or with HPLC technique are in good agreement with our findings of the dentist drill biopsies (Pegg,1984;Fischer,1981). Energy charges appear in close range indicating that the harvest time of the samples was nearly identical.

The pattern of the degradation products during catabolism of adenine nucleotides is not the same in all tissues. In contrast to the situation in heart tissue, the degradation of 
AMP in kidney does not result in accumulation of inosine and adenosine but mainly of hypoxanthine and, after prolonged ischemia, of xanthine (Van der Vusse,1984). These differences have been suggested to reflect a different susceptibility to ischemia (Gerlach,1971). Variable effects of degradation products have been reported. For instance, hypoxanthine may serve as a key substance in a resynthesis pathway of nucleotides (Buhl,1979). Furthermore, adenosine and inosine are known as vasoactive substances (Zager,1986). Empirical studies have shown that administration of some degradation products may exert a beneficial effect on postischemic recovery (Marshall,1985;Freeman,1986). However, the actual importance of in-vivo accumulated degradation products is still unknown.

The effect of ischemia on renal injury has been shown to be influenced by temperature. The beneficial effect of hypothermia in renal preservation at $4^{\circ} \mathrm{C}$ is most likely the result of reduced energy demand and decreased metabolic rate. The present study demonstrates that a decrease in temperature from $37^{\circ} \mathrm{C}$ to $32^{\circ} \mathrm{C}$ has a marked effect on the catabolic rate of the nucleotides. Lowering of the renal temperature in this range as may occur after circulatory arrest or during renal excision results in a significantly reduced degradation of AMP in comparison to breakdown during ischemia at body temperature. The pattern of the degradation products accumulated is, however, essentially similar, suggesting that the main effect of reduced temperature is lowering the catabolic rate of the adenine nucleotides.

The importance of small temperature changes has been recently demonstrated in a study in rats on renal ischemia tolerance during changes of whole body temperature (Maessen 1987d). A slight decrease of body temperature significantly reduced renal susceptibility to ischemia and the break down of adenine nucleotides. These data support our findings that even a small reduction of temperature affects the degradation rate of the latter substances. Data on the content of purine metabolites in human renal tissue are very limited. Recent studies using ${ }^{31}$ P NMR provided information on nucleotide behaviour in human kidney (Belzer,1983). The quantitation of the metabolites however is still cumbersome. In addition, only nucleotides and no degradation products can be monitored (Freeman,1986). Nevertheless, the NMR technique offers important advantages to conventional clinical methods such as the combined possibility to determine simultaneously $\mathrm{pH}$. In our study, human biopsies harvested during donor nephrectomy show adenine nucleotide levels similar to control values obtained in canine kidneys. The calculated ratio of degradation products and adenine nucleotides was $0.12 \pm 0.02$. If this value is interpreted according to the ischemia index diagram based on analysis of canine biopsies (fig. 3), it corresponds to a very short warm ischemia time which is in agreement with the clinical data on donor history.

It has been doubted whether wedge biopsies could be used for analysis of purine metabolites in the clinical setting. It was alternatively suggested that assessment of degradation products in the effluent of kidneys during wash-out may serve the same purpose (Takenaka,1980). However when ischemia time increases, effective flushing is reduced and the amount of accumulated degradation products is likely to be underestimated (Maessen,1986). Furthermore, cooling during the wash-out procedure may in itself influence the purine catabolism. Our experiments revealed that harvesting of biopsies from human kidneys was simple and safe. Basically, the technique used was similar to the harvesting of 'one hour' biopsies following implantation. Biopsies of $6 \mathrm{mg}$ wet weight were 
large enough for analysis of adenine nucleotides and their degradation products by HPLC.

Since reliable parameters of renal viability have not been definitively established in clinical practice, the duration of warm ischemia time is still the most important argument to accept or reject an ischemically affected donor kidney. In this study a method is presented to estimate the time duration of ischemia by analysis of the ratio of adenine nucleotides and their degradation products. It has been pointed out repeatedly that much larger ischemic intervals are tolerable than currently accepted, once appropriate preservation methods and reduced preservation time are applied (Kootstra,1986;Belzer,1983). A method as presented in this study that allows monitoring of ischemia time in kidneys with a suspicious donor history or a complicated donor nephrectomy may bring into consideration the use of otherwise discarded kidneys.

\section{SUMMARY}

To assess the warm ischemia time of kidneys with obscure donor history we attempted to develop in dog an index for the duration of ischemia by analysis of adenine nucleotides and their degradation products in renal cortical biopsies. Two biopsy harvesting techniques were compared. The use of a laboratory technique (dentist drill) resulted in higher concentrations of ATP in normoxic tissue specimen as compared to a clinical method to harvest biopsies (wedge biopsy). However the sum of adenine nucleotides (ATP, ADP, and AMP) was not significantly different between both groups $(\mathrm{p}<0.05)$. Therefore, wedge biopsies were used to study the degradation of adenine nucleotides following $0,30,60,90$, and 120 minutes of ischemia. Adenine nucleotides and their degradation products were assayed by HPLC technique. Concentrations of individual adenine nucleotides did not show a consistent correlation with warm ischemia time. However, as the sum of the adenine nucleotides (AN) and the sum of their degradation products (DP) decreased and increased respectively, the balance between these metabolites offered a good correlation with duration of warm ischemia.The ratio of DP to AN was significantly different at each interval $(P<0.05)$.

To study the influence of temperature on the degradation process, ischemia was induced at $37^{\circ} \mathrm{C}$ and $32^{\circ} \mathrm{C}$. Lowering of the temperature reduced the catabolic rate of the adenine nucleotides. The ratio DP and AN was significantly different from corresponding values at $37^{\circ} \mathrm{C}$.

In biopsies of non-ischemic human donor kidneys concentrations of adenine nucleotides and their degradation products were measured. Biopsies weighing less than $0.01 \%$ of total renal mass were large enough to meet analytical demands. The ratio of DP and AN in human kidney biopsies was in the same range as in the corresponding dog kidney biopsies.

These findings demonstrate that the ratio of DP to AN as determined from concentrations of purine metabolites in cortical wedge biopsies, is a sensitive and potentially useful index of warm ischemia time. 


\section{CHAPTER 5}

\section{INABILITY TO MAINTAIN ADENINE NUCLEOTIDE LEVELS BY COLD STORAGE IN ISCHEMICALLY DAMAGED AND CONTROL KIDNEYS ${ }^{1}$}

\section{INTRODUCTION}

The present study was designed to obtain insight in the effect of ischemia and hypothermia on renal adenine nucleotide metabolism. Reduction of the tissue content of adenine nucleotides and accumulation of their degradation products are important events in normothermic ischemic renal injury (Maessen,1988a). Since reduced levels of adenine mucleotides are thought to be related to loss of function of the renal cells, various measures have been developed to prevent the loss of these high energy phosphates in ischemic kidney. Hypothermia is the most conventional method. However, the protective effect of hypothermic storage is reduced if preceded by normothermic ischemia (Garvin,1985; Johnson,1972). We therefore analyzed the combined effect of normothermic ischemia and hypothermia on the levels of renal energy metabolites.test Two aims were addressed. First, to monitor the effect of temperature (ranging from $37^{\circ} \mathrm{C}$ to $4^{\circ} \mathrm{C}$ ) on the depletion of the sum of ATP, ADP, and AMP (AN) and the accumulation of their degradation products (IMP, adenosine, inosine, hypoxanthine, xanthine : DP). Secondly, to investigate the effect of cold storage at $4^{0} \mathrm{C}$ for 72 hours on the tissue content of AN and DP in kidneys flushed with Eurocollins after a period of normothermic ischemia. In addition, the efficiency of the observed Eurocollins wash out of degradation products from the ischemic renal tissue was determined by flushing kidneys in situ with blood following 30 and 90 minutes of normothermic ischemia. AN and DP were measured in tissue specimen from canine kidneys with the use of sophisticated HPLC techniques.

\section{MATERIALS AND METHODS}

Kidneys from adult mongrel dogs of both sexes weighing between 18 and $25 \mathrm{~kg}$ were used $(\mathrm{N}=47)$. For biochemical analysis tissue specimen were obtained from the renal cortex with a surgical blade and rapidly frozen in liquid nitrogen. In a first series of experiments to study the effect of hypothermia by surface cooling, kidneys were removed immediately after cross-clamping and stored at a constant environmental temperature at $32^{\circ} \mathrm{C}, 24^{0} \mathrm{C}$, or $4^{0} \mathrm{C}(\mathrm{N}=5$ in each group $)$. Normothermic $\left(37^{\circ} \mathrm{C}\right)$ control values were obtained from kidneys that were left in situ. Normothermic ischemia was induced in situ by cross-clamping of the renal vessel pedicle. During the ischemic interval the abdomen was closed and a thermostatic watermattress was used for temperature control of the body $(\mathrm{N}=6)$. Biopsies were taken at 30 minute intervals for 90 minutes.

In a second series of experiments kidneys were removed following 30 and 90 minutes of normothermic ischemia in situ and subsequently flushed with Eurocollins at $4^{\circ} \mathrm{C}$ during 8 minutes ( $N=5$ in each group). Following the flushing procedure the kidneys were stored on ice during 72 hours. Biopsies were taken just before and after Eurocollins flushing and after storage at $4^{\circ} \mathrm{C}$ for 72 hours. To study the efficiency of the hypother-

Based on: Maessen JG, van der Vusse GJ, Vork M, Kootstra G. Transplant proc 19:4112-4115,1987. 
mic flushing with the Eurocollins solution, kidneys were rendered ischemic at $37^{0} \mathrm{C}$ for 30 and 90 minutes and were subsequently lylreperfused in situ with blood at $37^{\circ} \mathrm{C}$ for another 5 minutes. Levels of AN and DP were determined by HPLC as described before (Wynants,1985). Statistical analysis was performed by the Wilcoxon rank-sum test. P values less than .05 were considered to be significant.

\section{RESULTS}

The effect of hypothermia on the ischemia-induced reduction of AN levels is already

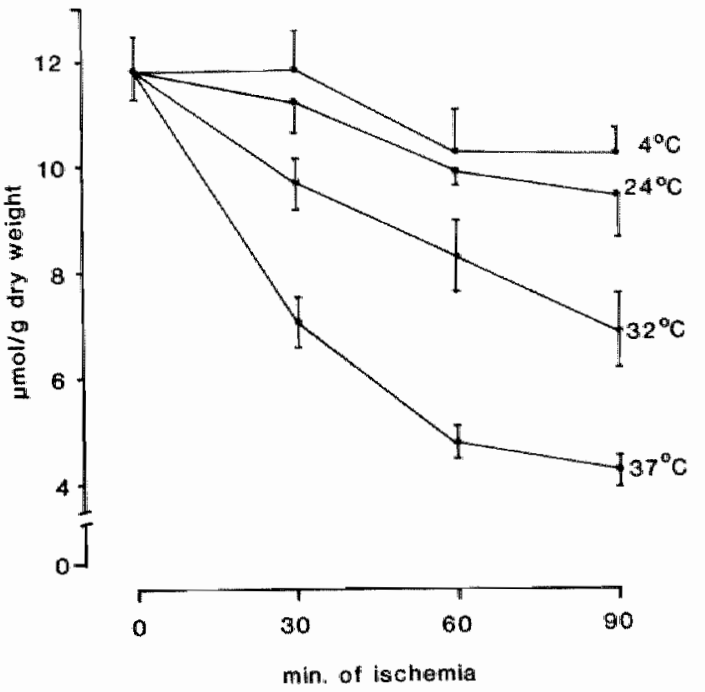

Figure 5.1.: The effect of temperature on the depletion of the tissue content of adenine nucleotide (AN) during renal ischemia.. Kidneys were rendered ischemic at $37^{\circ} \mathrm{C}, 32^{0} \mathrm{C}, 24^{\circ} \mathrm{C}$, and at $4^{0} \mathrm{C}$. Cortical biopsies were taken with a surgical blade. Biochemical determinations were performed with an HPLC assay. Data were ex* pressed as mmol/g dw and presented as means $\pm S E M$.

substantial within the first $30 \mathrm{~min}$ of ischemia (see figure 5.1). During normothermic ischemia AN content drops to $36 \%$ of control after 90 minutes. Lowering temperature to $32^{\circ} \mathrm{C}$ and $24^{\circ} \mathrm{C}$ resulted in $\mathrm{AN}$ levels of $58 \%$ and $79 \%$ of pre-ischemic control values respectively. Cooling at $4^{\circ} \mathrm{C}$ did not further improve preservation within 90 minutes of ischemia as compared with $24^{\circ} \mathrm{C}$. It is shown figure 5.2 that the protective effect of temperature during ischemia on the AN pool is mainly the result of reduced AMP degradation. The sum of ATP and ADP similarly decreased in hypothermic and normothermic ischemia to 1.64 and $1.37 \mu \mathrm{mol} / \mathrm{g} \mathrm{dw}$ after 90 minutes, respectively (data not shown). During flushing with Eurocollins at $4^{\circ} \mathrm{C}$ after normothermic ischemia for 30 and 90 minutes no AN were lost from the affected kidneys (table 5.1). Loss of DP from the Eurocollins perfused kidneys was $66 \%$ (30 min group) and $67 \%$ ( 90 min group) of the amount of DP that accumulated during the preceding ischemic episode. If kidneys were 
reperfused in situ with blood the content of DP was found to be reduced to $24 \%$ ( $30 \mathrm{~min}$ group) and $36 \%$ (90 min group) of the ischemic values. Eurocollins flushing of kidneys without prior normothermic ischemia slightly decreased AN with a concomitant small increase of DP. However, these values did not reach a level of significance. The results presented in table 5.1 clearly indicate that simple cold storage at $4^{0} \mathrm{C}$ during 72 hours was unable to prevent the loss of $\mathrm{AN}$ in control kidneys and in kidneys that were previously rendered ischemic for 30 minutes. After 72 hours the content of $A N$ and DP was nearly similar in all three experimental groups. It should be noted that the already low levels in the kidneys previously subjected to prolonged ischemia for 90 minutes remained unaffected during cold storage.

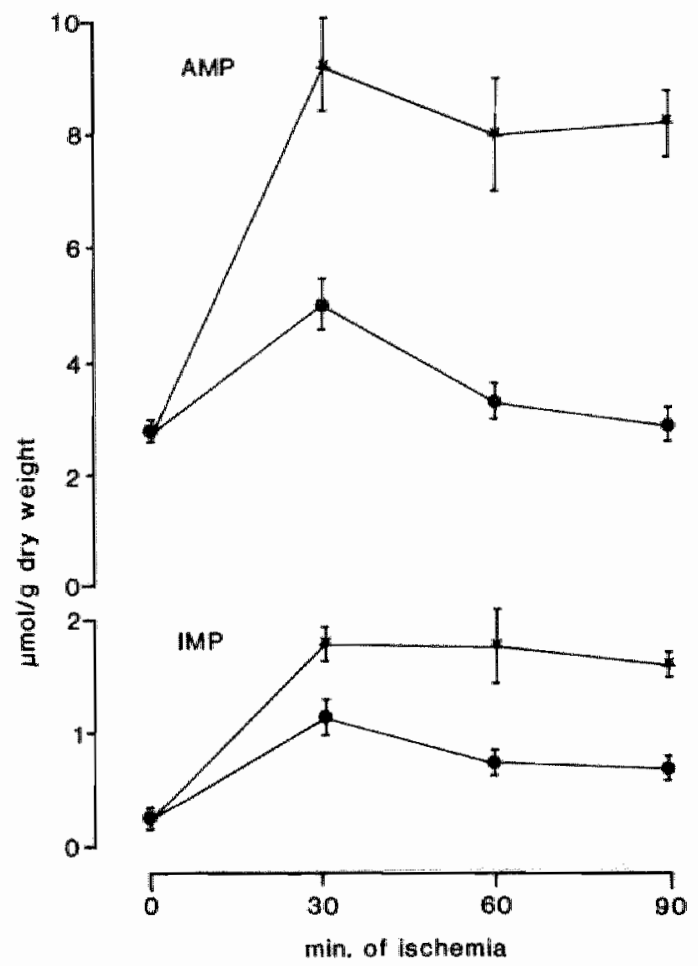

Figure 5.2: The catabolic profile of AMP and IMP during ischemia at $37^{0} \mathrm{C}(\boldsymbol{c})$ and $4^{0} \mathrm{C}(\boldsymbol{t})$. All ischemic values obtained at $37^{\circ} \mathrm{C}$ were significantly different from walues at $4^{0} \mathrm{C}(\mathrm{p}<0.05)$. See further legend of figure 5.1

\section{DISCUSSION}

Hypothermic preservation has been successfully used to increase ischemia tolerance in donor organs. The mechanism of action of hypothermia as a defense strategy against ischemic damage is based on the concept that the imbalance between energy demand and energy supply during ischemia is reduced by lowering metabolic rate and hence conservation of high energy metabolites. However, if normothermic ischemia precedes hypothermic preservation, the efficiency of cooling is markedly reduced (Garvin,1985; 
Johnson, 1972). We therefore documented changes of adenine nucleotides and their degradation products during hypothermia with or without prior normothermic ischemia in order to determine the role of high levels of adenine nucleotides to the ischemia protective effect of hypothermia. It was demonstrated that normothermic ischemia rapidly exhausts the pool of high energy phosphates. Within 90 minutes only one third of the adenine nucleotide content is left in cortical renal tissue. Simultaneously a profound increase of degradation products is found, mainly consisting of hyopxanthine and xanthine. Hypothermia in the initial phase of ischemia, as is shown in the surface cooling experiments may strikingly reduce this AN break down. As the largest drop of AN occurs in the first 30 minutes of normothermic ischemia it might be obvious that the most rapid cooling method may preserve the largest amount of AN. Our results indicate however that lowering temperature from $24^{\circ} \mathrm{C}$ to $4^{\circ} \mathrm{C}$ does not significantly improve the maintenance of high $\mathrm{AN}$ levels. These findings give support to the concept that higher preservation temperatures may give equal or maybe better results than storage at $4^{0} \mathrm{C}$ (Wickham,1967;Pegg,1985). The most important step in the hypothermia modified catabolism of AN is the conversion of AMP. Since it is generally supposed that AMP is dephosporylated to adenosine by 5 '-nucleotidase it appears that hypothermia particularly affects the activity of this enzyme (Busch,1968;Buhl,1979). Inhibition of 5'-nucleotidase also reduces dephosphorylation of IMP. As the level of IMP was found to be increased by hypothermia our data imply that at least part of AMP is deaminated by adenylic acid deaminase to IMP. Cooling of kidneys by flushing at $4^{\circ} \mathrm{C}$ has long been considered the most effective way of starting preservation. Evidence has been gathered however that this method bears some disadvantages with similar symptoms as in the cold shock syndrome (Jacobsen,1982). In the present study comparison of the removal of DP by Eurocollins flushing to the wash out by in situ blood reperfusion suggests that flushing at $4^{\circ} \mathrm{C}$ is incomplete and can be improved. Our data are in concert with earlier reports in which an incomplete flow distribution of Eurocolins in renal tissue was demonstrated (Maessen,1986; Foreman,1982).

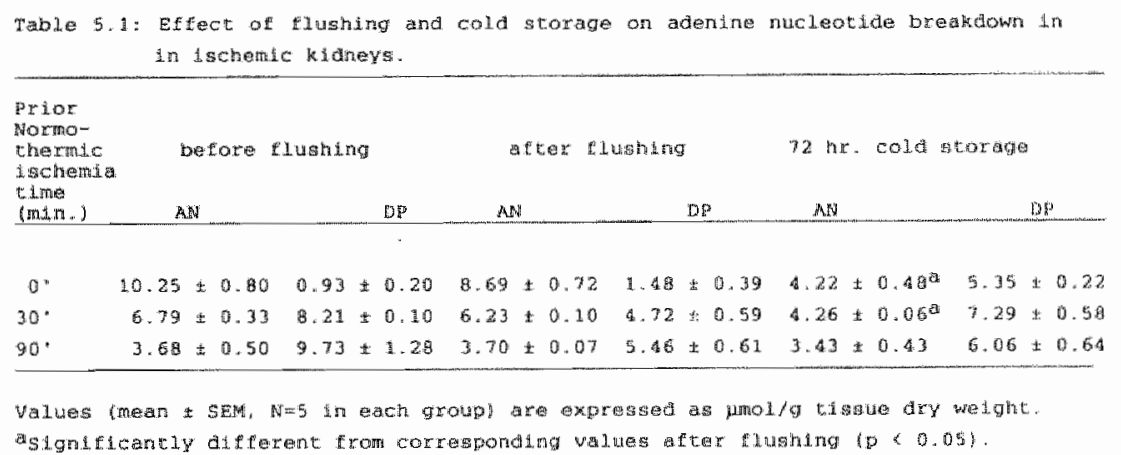

Cold storage at $4^{0} \mathrm{C}$ for 72 hours was found to smooth out the differences between the AN content of ischemically damaged and control kidneys. The severely decreased pool of AN in kidneys after prolonged normothermic ischemia ( 90 minutes) did not further deplete during 72 hours of cold storage. In kidneys without considerable normothermic 
ischemia, AN break down continued during cold storage to values that eventually, closely approximated the vallues of the severe ischemically damaged kidneys. Thus the effect of hypothermia on the tissue content of $\mathrm{AN}$ at the end of three days cold storage did not reflect the influence of prior normothermic ischemia. Newly developed preservation techniques attempt to support the hypothermic energy metabolism by providing precursors for AN resynthesis or by preventing depletion of AN with drugs, based on the assumption that high levels of AN maintain renal viability during preservation (Garvin,1985; Pegg,1984;Southard,1984). Our present data however suggest that depletion of adenine nucleotide content is not the sole factor that limits the efficiency of hypothermic ischemia protection. 


\section{CHAPTER 6}

\section{NEW APPROACH TO PRESERVATION OF NON-HEART BEATING DONOR KIDNEYS: TOTAL BODY COOLING ${ }^{1}$}

\section{INTRODUCTION}

Rapid cooling of kidneys from non-heart beating donors to prevent ischemic injury is essential but not always possible for technical and legal reasons (Kootstra,1986). Since the main objective of preservation is to decrease the rate of energy consuming metabolic processes by hypothermia, we studied the efficiency of total body cooling as a noninvasive preservation method to bridge the time before nephrectomy or in situ cooling of the kidney can be started. To monitor the protection of cooling against ischemia adenine nucleotide content was measured in rat kidneys following cadaver storage at various temperatures. ATP, ADP, and AMP were measured since changes in the content of these substances are determined by ischemia time (Maessen,1987a).

\section{MATERIALS AND METHODS}

Wistar rats of both sexes weighing between 250 and $300 \mathrm{~g}$ were used. Animals were anaesthetized with ether and rapidly killed by cervical dislocation. An indwelling thermistor probe was adjusted into the rectum to monitor continuously body temperature. The cadavers were stored at various temperatures using a thermostated oven and ice. The animals were assigned to five experimental group; group 1, storage at $38^{\circ} \mathrm{C}(\mathrm{N}=23)$, group 2, storage at $24^{0} \mathrm{C}(\mathrm{N}=23)$, group 3 , storage at $4^{0} \mathrm{C}(\mathrm{N}=24)$, group 4 , storage on melting ice ( $\mathrm{N}=16$ ), and group 5 , storage on melting ice after Eurocollins flushing $(\mathrm{N}=11)$. Kidneys were flushed with Eurocollins at $4^{6} \mathrm{C}$ during 8 minutes. After 60 minutes of storage on ice they were rapidly frozen by freeze-clamping in liquid nitrogen for biochemical analysis. In the other groups kidneys were harvested either after 30 or 60 minutes ischemia and subsequently frozen in liquid nitrogen. Control values were determined in fresh kidneys from 12 animals. Biochemical determination of ATP, ADP, and AMP was performed with a HPLC method (Wynants,1985). Values are expressed as $\mu \mathrm{mol}$ per gram of dry weight. Total adenine nucleotide content was determined by summing ATP, ADP, and AMP. Significance of differences between data was determined by the Wilcoxon rank-sum test. A p value of less than .05 was considered to be statistically significant.

\section{RESULTS}

The content of adenine nucleotide content of fresh rat kidneys was $14.88 \pm 0.35 \mu \mathrm{mol} /$ $\mathrm{g} \mathrm{dw}$. Without cooling the loss of adenine nucleotides amounted from $64 \%$ after 30 minutes to $75 \%$ after 60 minutes of ischemia. Immediate cooling with in situ Eurocollins perfusion and subsequent storage of the kidneys on ice reduced adenine nucleotide depletion to $22 \%$ after 60 minutes. Body cooling in group 2, 3, and 4 decreased the nucleotide content with $65 \%, 61 \%$, and $45 \%$ after 30 minutes, and with $73 \%, 68 \%$, and $45 \%$ 
after 60 minutes, respectively. The values of group 3,4 , and 5 were significantly differ-

\begin{tabular}{|c|c|c|c|c|c|c|}
\hline Grotip & (1) $)$ & 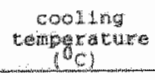 & $\begin{array}{l}\text { scoragte } \\
\text { (tanting) }\end{array}$ & $\begin{array}{c}\text { rectal } \\
\text { exmperaty } \\
0 \mathrm{cl}\end{array}$ & $\begin{array}{l}\text { cooling: } \\
\text { rate } \\
\text { ac/matn }\end{array}$ & $\begin{array}{c}\text { adenine } \\
\text { nucleotides } \\
\text { thmol } / \text { d dw }\end{array}$ \\
\hline 1. & $\begin{array}{l}12 \\
11\end{array}$ & $\begin{array}{l}315 \\
38\end{array}$ & 30 & $\begin{array}{l}38.0 \\
38.0\end{array}$ & -- & $\begin{array}{l}5.39 \pm 0.26 \\
3.72 \pm 0.159\end{array}$ \\
\hline$z$ & $\begin{array}{l}12 \\
12 \\
11\end{array}$ & $\begin{array}{l}24 \\
24\end{array}$ & $\begin{array}{l}30 \\
600\end{array}$ & $\begin{array}{l}35.1 \\
32.8\end{array}$ & 0.08 & $\begin{array}{l}5.15 \pm 0.20 \\
3.98 \pm 0.1 .4\end{array}$ \\
\hline 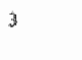 & $\begin{array}{l}6 \\
10\end{array}$ & $\begin{array}{l}1 \\
4\end{array}$ & $\begin{array}{l}30 \\
40\end{array}$ & $\begin{array}{l}30.5 \\
26.6\end{array}$ & 0.15 & $\begin{array}{l}5.86 \pm 0.51 \\
4.66 \pm 0.18^{b}\end{array}$ \\
\hline 4 & $\begin{array}{l}6 \\
30\end{array}$ & $\begin{array}{l}0 \\
0\end{array}$ & 30 & $\begin{array}{r}21.8 \\
9.2\end{array}$ & 0.48 & $\begin{array}{l}3.22 \pm 0.650 \\
3.06 \pm 0.644^{50}\end{array}$ \\
\hline$y^{\mathbb{a}}$ & in & 0 & 60 & 0.0 & 0.63 & $1.1 .54 \pm 0.276$ \\
\hline
\end{tabular}

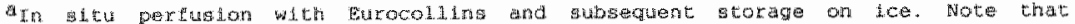

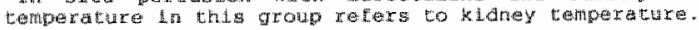

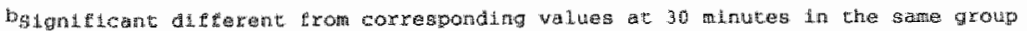
$(p<0.05)$

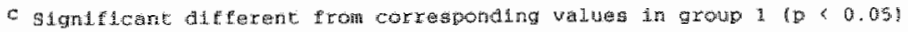

ent in comparison to group 1 at $\mathrm{p}<0.05$ (see table 6.1). As the adenine nucleotide levels in group 4 were not different after either 30 or 60 minutes, the cooling rate of the cadavers stored on ice was apparently fast enough to arrest further adenine nucleotide break down following 30 minutes of total body hypothermia.

\section{DISCUSSION}

At present, the development of organ procurement programs to enlarge the supply of donor organs may contribute more substantially to increase the number of successful transplantations than further improvement of anti-rejection therapy. One way to counteract the shortage of donor organs is offered by harvesting organs from non-heartbeating donors.

The use of non-heartbeating donors however is still discouraged by two major problems. First, ats the development of circulatory collapse is generally a fluctuating process the onset of the ischemic interval is difficult to estimate. Hence the degree of ischemic injury to the kidney remains obscure and may have been detrimental to renal viability. The development of parameters to assess ischemic injury to renal tissue during preservation may in part solve this problem (Maessen,1987a). Secondly it is still illegal to perform a groin incision for introduction of a double-balloon catheter, required for rapid flushing and cooling of the kidney to prevent warm ischemic injury, in human cadavers before permission for organ donation has been obtained. As potential non-heartbeating donors are often presented in emergency situations it may take too much time before relatives can be consulted to give their permission for organ harvesting. Therefore a non-invasive and at any time applicable method to initiate hypothermic protection against renal ische- 
mia would render deceased non-heartbeating patients more suitable as organ donors. With this objective in mind, the present experimental study in rat was performed to examine the possibilities of total body cooling as a non-invasive method to initiate the preservation of donor kidneys. Cold preservation is generally based on the concept that hypothermia can reduce the energy demand in renal tissue during ischemia-elicited impairment of the energy metabolism (Hochachka,1986). We therefore measured the renal content of adenine nucleotides following cadaver storage at various temperatures. Immediate cooling of the kidneys with an invasive technique such as Eurocollins perfusion at $4^{\circ} \mathrm{C}$, followed by storage on melting ice resulted in an excellent preservation of renal adenine nucleotide levels. Adequate preservation of the tissue content of these substances was observed in case of total body cooling on melting ice. Interestingly, the drop in body temperature appeared to be gradually, i.e. $0.48^{\circ} \mathrm{C} / \mathrm{min}$. The loss of renal tissue adenine nucleotides was found to be about $50 \%$ of values obtained in fresh kidneys. Storage of the body at $4^{\circ} \mathrm{C}$ and $24^{\circ} \mathrm{C}$ resulted in considerably less effective maintenance of the tissue content of adenine nucleotide. The potential beneficial effect of preventing adenine nucleotide break down by total body cooling on melting ice to renal recovery following transplantation has still to be proven. In a recent study however it has been demonstrated that effective body cooling to some degrees below normal can arrest ischemic catabolism of energy metabolites and improve postischemic renal recovery in situ (Zager,1986).

In conclusion, although in situ perfusion with Eurocollins remains the most effective way for preservation of kidneys from non-heart beating donors, simple body cooling can significantly reduce the detrimental effect of ischemia on renal energy metabolism. The present results warrant further exploration since a simple, non-invasive, and juridically acceptable method of preservation will stimulate harvesting of non-heart beating donor kidneys.

\section{SUMMARY}

In non-heart beating kidney donors, rapid cooling to prevent ischemic injury is essential but not always possible for technical or legal reasons. We studied the effectiveness of total body cooling as a simple and non-invasive method to initiate preservation of renal adenine nucleotide levels before nephrectomy or in-situ cooling can be started. Wistar rats were killed by cervical dislocation. The cadavers were stored at $38^{\circ} \mathrm{C}$ (group I), $24^{\circ} \mathrm{C}$ (group II), $4^{\circ} \mathrm{C}$ (group III), and $0^{\circ} \mathrm{C}$ (melting ice, group IV). The effect of body temperature to renal ischemia was compared with the effect of immediate in-situ perfusion with Eurocollins $\left(4^{\circ} \mathrm{C}\right.$ ) after cervical dislocation, with subsequent storage on melting ice of the isolated kidneys (group V). Body temperature was monitored with a rectal thermistor probe. Kidneys were freeze clamped 60 minutes after the onset of storage. The extent of renal ischemia was assessed by measuring the depletion of the adenine nucleotide pool (ATP, ADP, and AMP) analyzed with an HPLC technique. Body temperature decreased to $32.8^{\circ} \mathrm{C}, 26.6^{\circ} \mathrm{C}$, and $9.2^{\circ} \mathrm{C}$ in group II, III, and IV respectively during $60 \mathrm{~min}$ of storage. Without cooling the loss of adenine nucleotides (AN) amounted from 14.( to $3.7 \mu \mathrm{mol} / \mathrm{g} \mathrm{dw}$ after 60 minutes. Immediate cooling with in-situ perfusion preserved AN levels to $11.5 \mu \mathrm{mol} / \mathrm{g} \mathrm{dw}$ after 60 minutes. Cooling of the body in group II, III, and IV reduced AN levels after 60 minutes to $4.0,4.7$, and $8.0 \mu \mathrm{mol} / \mathrm{g}$ dw respectively. These differences were all significant at $\mathrm{p}<0.05$. In conclusion, although in-situ perfusion with 
Eurocollins remains the most effective way for preservation of kidneys from non-heart beating donors, simple body cooling can reduce significantly the detrimental effect of ischemia to renal energy metabolites as well. These findings may have important clinical relevance as a simple and juridically acceptable method of preservation will stimulate harvesting of non-heart beating donor kidneys. 


\section{CHAPTER 7}

\section{POTENTIATION OF ISCHEMIC INJURY BY HYPOTHERMIA IN DONOR KID- NEYS: ROLE OF THE ENERGY METABOLISM ${ }^{1}$}

\section{INTRODUCTION}

Long-term cold storage of donor kidneys following a period of normothermic ischemia has been considered to be deleterious to posttransplant renal function (Pegg, 1985). A possible role of tissue adenine nucleotides (AN) in the inability of the kidney to recover after transplantation following cold storage has not yet been established. In a previous study we have demonstrated that the extent of AN depletion in kidney during storage at $4^{0} \mathrm{C}$ is dependent on the AN level at the beginning of the hypothermic period (Maessen, 1987). After $90 \mathrm{~min}$ of normothermic ischemia which reduced the AN level to about $35 \%$ no further depletion of AN occurred during subsequent cold storage for 3 days, whereas after 30 min of ischemia with AN levels reduced to $66 \%$ of control, as well as in nonischemic kidneys with normal AN levels, a depletion was found to $41 \%$ of control after 3 days of cold storage. These findings suggest that AN levels during prolonged cold storage will not drop below 35 - 40\% of control, irrespective of the duration of the preceding period of normothermic ischemia. The time interval of normothermic ischemia has been found to influence substantially the outcome of kidney function, transplanted after long-term cold storage. Hence the level of $\mathrm{AN}$ at the end of the cold storage seems not to be a decisive factor for posttransplant renal viability.

The question was then raised whether the recovery of the AN stores in posttransplant kidneys is influenced by cold storage following normothermic ischemia of variable duration. The present study was conducted to address this question. Tissue specimens were taken from the cortex of canine kidneys subdued to 30 and 90 minutes of normothermic ischemia, after 48 hours of cold storage, and at the end of 60 minutes of reperfusion following transplantation. In control experiments the period of cold storage was omitted and kidneys were reperfused in situ. With a sophisticated HPLC technique, the content of adenine nucleotides (ATP, ADP, AMP) and degradation products such as IMP, adenosine, inosine, hypoxanthine, and xanthine, was determined.

\section{MATERLALS AND METHODS}

Kidneys from adult mongrel dogs of both sexes were used. Normothermic ischemia was induced by cross-clamping of the renal vessel pedicle of the left kidney. The abdomen was closed during the ischemic episode and the temperature of the body was kept constant using a thermostatic watermattress. In situ reperfusion by removing of the vessel clamp was preceded by nephrectomy of the contralateral kidney. If hypothermically stored, the kidneys were removed after the ischemic period and flushed with Eurocollins at $4^{\circ} \mathrm{C}$ during $8 \mathrm{~min}$. Kidneys were stored on ice during 48 hours. Autologous re-implantation, taking 20 to $30 \mathrm{~min}$, was performed in the abdomen using the iliac vessels. The contralateral kidney was removed before reinstallment of the blood circulation. Following one hour reperfusion the abdomen was closed and the animals were allowed to

Based on: Maessen JG, wan der Vusse G.I, Vork M, Kootstra G. Transplant proc, in press. 
recover. The animals were alloted to four experimental protocols. In all groups $(\mathrm{N}=6$ in each group) ischemia was induced; in group I and III during 30 min and in group II and IV during $90 \mathrm{~min}$. In group I and II ischemia was followed by in situ reperfusion. In group III and IV kidneys were subjected to cold storage after ischemia.

For determination of the levels of energy metabolites, biopsies were obtained from renal cortical tissue with a surgical blade. Tissue specimens were rapidly frozen in liquid nitrogen and stored at $-80^{\circ} \mathrm{C}$ until further analysis. Biopsies were taken at the end of the ischemic interval, at the end of the cold storage period, if any, and at one hour reperfusion. The levels of energy metabolites were determined by HPLC as described before (Wynants,1985). The adenylate energy charge was calculated as (ATP + 1/2 ADP)/ (ATP + ADP + AMP). Adenine nucleotide (AN) levels were defined as the sum of ATP, ADP, and AMP. Values were given in $\mu \mathrm{mol} / \mathrm{g}$ dry weight of tissue and were expressed as means \pm SEM. Statistical analysis was performed by the Wilcoxon rank-sum test. $P$ values less than 0.05 were considered to be significant.

\section{RESULTS}

After $30 \mathrm{~min}$ of ischemia and immediate in situ reperfusion an increase of $3.1 \mu \mathrm{mol}$ AN

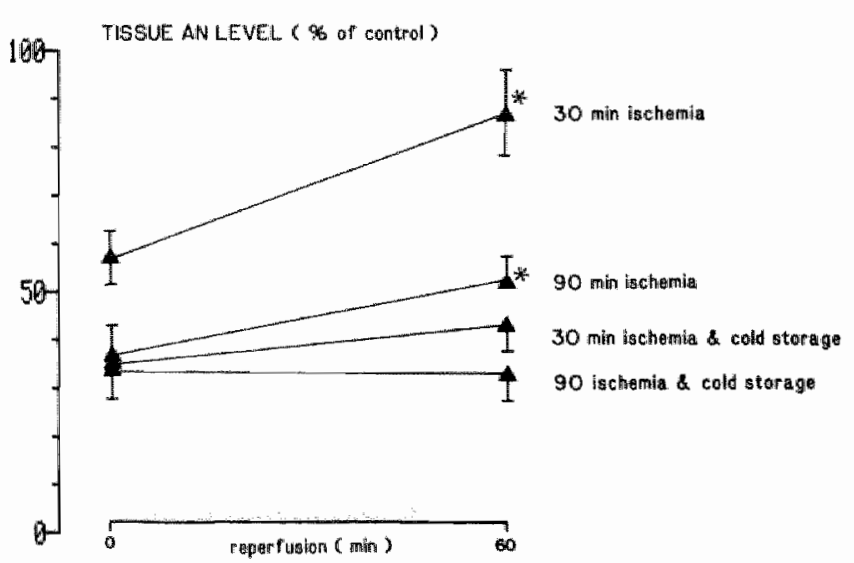

Figure 7.1.: Tissue AN levels expressed as percentage of pre-ischemic controls during reperfusion. Data are given as mean \pm SEM. Asterisks indicate a significant difference from corresponding values at time zero of reperfusion. i.e. values at the end of the ischemic interval or the period of cold storage $(p<0.05)$.

per $g \mathrm{dw}$ was found versus $1.61 \mu \mathrm{mol}$ AN per $g$ dw following $90 \mathrm{~min}$ (significant at $\mathrm{p}<$ 0.05 ) (figure 7.1). Kidneys that were hypothermically stored after normothermic ischemia (either 30 or $90 \mathrm{~min}$ ) did not show a significant increase of adenine nucleotides within $60 \mathrm{~min}$ reperfusion. The depletion of $\mathrm{AN}$ after $30 \mathrm{~min}$ of ischemia continues during cold storage from $6.3 \pm 0.2$ to $3.9 \pm 0.3 \mu \mathrm{mol} / \mathrm{g} \mathrm{dw}(\mathrm{p} 0.05$ ) whereas after $90 \mathrm{~min}$ of ischemia no significant depletion was found during cold storage ( $4.1 \pm 0.5$ vs. $3.8 \pm 0.4$ 

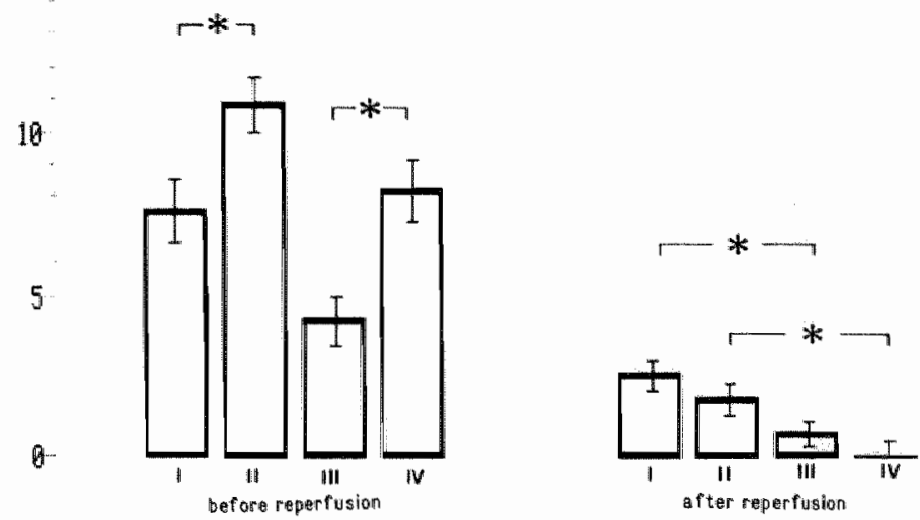

Pigure 7.2.: The renal tissue level of degradation products (sum of IMP, adenosine, inosine, hypoxanthine, and xanthine) immediately before reperfusion and at the end of reperfusion. Values are given in $\mu \mathrm{mol} / \mathrm{g}$ dw and expressed as means \pm SEM. I, II, III, and IV refer to the following experimental groups: $I_{4}, 30$ min of ischemia; II, 90 min of ischemia; III, 30 min of ischemia followed by cold storage for 48 hours; IV, 90 min of ischemia followed by cold storage for 48 hours. Asterisks indicate a significant difference between the groups indicated $(p<0.05)$

$\mu \mathrm{mol} / \mathrm{g} \mathrm{dw}$ ) (figure 7.1). Concomitant to increased adenine nucleotide depletion after $90 \mathrm{~min}$ of ischemia a significant larger amount of degradation products was found in these kidneys in comparison to kidneys following $30 \mathrm{~min}$ of ischemia (figure 7.2). In

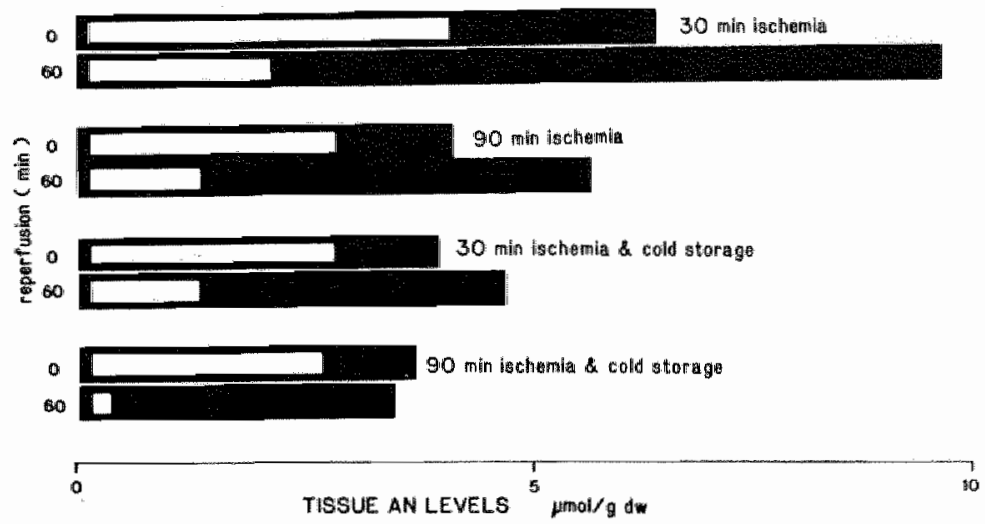

Figure 7.3: The effect of reperfusion on the content of ATP + ADP (black bars) and AMP (open bars) in kidney cortex. Mean values are shown. 
hypothermically preserved kidneys a similar difference was found. The accumulation of degradation products, however, was significantly less than in kidneys without cold storage. Hypoxanthine was the mayor compound of the degradation products present in kidney tissue before reperfusion. IMP and xanthine accumulated to a smaller extent. Inosine and adenosine levels were present in minor amounts (data not shown). At the end of the reperfusion time the tissue level of degradation products returned to control values or to values below control (i.e. $2.5 \pm 0.2 \mu \mathrm{mol} / \mathrm{g} \mathrm{dw}$ ). Only in kidneys with a high adenine nucleotide recovery following reperfusion ( $30 \mathrm{~min}$ of ischemia) the levels of degradation products were significantly higher than in kidneys from the other groups. In both cold storage groups the levels of the degradation products in reperfused kidneys were significantly less than in pre-ischemic control biopsies. The effect of normothermic ischemia with and without 48 hours cold storage on the balance of ATP, ADP, and AMP before and after 60 min reperfusion is shown in figure 7.3. In all groups an increase of the sum of ATP and ADP was found during reperfusion. In group I and II kidneys reperfused without cold storage after 30 and 90 minutes of normothermic ischemia, respectively, the increase in the sum of ATP and ADP exceeded significantly the decrease in AMP. In contrast, in the groups with cold storage, the increase in tissue levels of ATP + ADP exactly matched the fall in AMP. The adenylate energy charge values were not significantly different (group I, $0.58 \pm 0.03 ;$ II, $0.56 \pm 0.04 ;$ III, $0.51 \pm 0.05 ;$ IV, $0.57 \pm 0.04$ ).

\section{DISCUSSION}

The present data show that following ischemia and in situ reperfusion without intermediate cold storage, the recovery rate of $\mathrm{AN}$ is related to the duration of the preceding, normothermic ischemic period. The AN recovery rate during one hour of reperfusion is found to be 0.052 and $0.026 \mu \mathrm{mol}$ per min per $\mathrm{g}$ tissue $\mathrm{dw}$ following 30 and $90 \mathrm{~min}$ of ischemia, respectively. When reperfusion is preceded by cold storage for 48 hours no net recovery of $\mathrm{AN}$ can be demonstrated within $60 \mathrm{~min}$ after reinstallment of the flow neither in the kidneys subjected to $90 \mathrm{~min}$, nor in those subjected to $30 \mathrm{~min}$ of ischemia. The extent of adenine nucleotide depletion under ischemic conditions has been regarded as a decisive factor for the inability to recover the nucleotide pool upon reperfusion (Callman,1979; Chaudry,1983; Gaudio,1986). In situ reperfusion studies show a larger nucleotide resynthesis in those kidneys with the smallest ischemic nucleotide loss. Comparison of these results with the reperfusion studies following cold storage, however, reveals that the nucleotide level per se is not necessarily related to the capacity of nucleotide resynthesis at the onset of reperfusion. It is interesting to note that the depletion of adenine nucleotides following $90 \mathrm{~min}$ of ischemia is similar in kidneys with and without 48 hours cold storage whereas nucleotide resynthesis within 1 hour reperfusion is only observed in kidneys without cold storage. The effect of the combination of cold storage and normothermic ischemia suggests that the level of adenine nucleotide depletion before reperfusion is not related to the degree of adenine nucleotide recovery during reperfusion. There are various ways to replenish the depleted AN pool in postischemic tissue. In addition to de novo synthesis, a process which requires a considerable amount of energy, the salvage pathway using hypoxanthine as substrate has been considered as an important mechanism to restore the AN pool (Murray,1971). The present findings indicate that the amount of degradation products, including hypoxanthine, is considerably smaller in kidneys stored at $4^{\circ} \mathrm{C}$ for 48 hours than in the corre- 
sponding ischemic organs not subjected to cold storage (figure 7.2). A previous study has shown that this observation can in part be explained by the effect of the treatment with Eurocollins in between the normothermic ischemic insult and the storage period (Maessen,1988m). Eurocollins caused a wash-out of a significant part of degradation products accumulated in the kidney during the preceding ischemic episode. In line with the previous findings is the present observation that the tissue level of degradation products following one hour of reperfusion is significantly higher in kidneys subjected to ischemia allone than in those in which ischemia was combined with cold storage after Eurocollins flushing (figure 7.2, right panel). It is tempting to speculate that the endogenous store of purine degradation products is a source of resynthesis of the AN pool after reinstallment of the blood flow.

Data presented in figure 7.3 indicate that the ability to restore high-energy adenine nucleotides from AMP has not been destroyed in the postischemic kidneys. Both in kidneys subdued to ischemia alone and to ischemia in combination with cold storage, AMP levels dramatically fell after restoration of blood flow. As a consequence the energy charge in these kidneys returned to its pre-ischemic value. This observation supports the notion that the inability to replenish endogenous $\mathrm{AN}$ stores in previously ischemic kidneys subjected to Eurocollins treatment and prolonged cold storage might be due to a substantial lack of appropriate substrate for AN resynthesis during the reperfusion phase. 


\section{CHAPTER 8}

\section{ASSESSMENT OF NUUCLEOTIDES, NUCLEOSIDES, AND OXYPUIRINES IN HUMAN KIDNEYS WITH THE USE OF REVERSED-PHASE HIGH PERFORMANCE LIQUID CHROMATOGRAPHY ${ }^{1}$}

\section{INTRODUCTION}

Tissue levels of adenine nucleotides are commonly monitored to evaluate the effect of ischemia and the adequacy of preservation techniques on the energy status of kidneys used for transplantation in experimental studies (Takenaka, 1980; Pegg,1985; Garvin,1985; Buhl,1979; Calman,1974). The introduction of a reliable method applicable to the clinical setting to determine tissue levels of adenine nucleotides and related substances in human kidneys has not yet been established although the need is clearly present. Basically two different approaches can be followed. First, a non-invasive technique using ${ }^{31}$ P NMR spectroscopy. A drawback of this technique is the incompleteness of information that is obtained. Only nucleotides are measured whereas important degradation products such as purine bases and oxypurines remain invisible. In addition, the coil size of the currently applied NMR apparatus does not allow in situ measurements of human kidneys (Marshall,1985; Freeman,1986). Second, invasive techniques comprising collection of biopsies followed by chemical assay techniques appear to be an attainable alternative. In stead of applying time-consuming enzymatic assay techniques we have evaluated a high performance liquid-chromatography technique to measure the content of ATP, ADP, AMP, GTP, IMP, adenylic succinate, adenosine, inosine, hypoxanthine, xanthine, xanthosine, guanosine, XMP, and uric acid in tissue specimen of human kidneys. This method is now routinely used in our laboratory to screen the energy status in human renal biopsies that are obtained during donor nephrectomy, at the end of preservation time, and one hour following implantation (Maessen,1988a).

\section{MATERIALS AND METHODS}

\section{Human biopsies}

Human donor kidneys were routinely biopsied with a surgical blade. The first biopsy was taken during donor nephrectomy before cessation of the blood circulation. A second and third set of biopsies were harvested at the end of the hypothermic storage period and one hour after restoration of the circulation during implantation, respectively. Biopsies were immediately frozen in liquid nitrogen.

Sample preparation

All cortical tissue samples were stored at $-80^{\circ} \mathrm{C}$. Before analysis the tissue samples were lyophilized at $-30^{\circ} \mathrm{C}$. Adherent blood was removed. After weighing, the tissue samples ( 1 - $7 \mathrm{mg}$ tissue dry weight) were extracted with $300 \mu \mathrm{L}$ extraction mixture containing $1.5 \mathrm{M} \mathrm{HCLO}_{4}$ and $5 \mathrm{mM}$ dithiotreitol. A volume of $200 \mu \mathrm{L}$ of this extract was frozen in liquid nitrogen. The frozen extract was submerged in $150 \mu \mathrm{L} \mathrm{KHCO}_{3}(2 \mathrm{M})$. The tissue extract- $\mathrm{KHCO}_{3}$ mixture was allowed to thaw during centrifugation at $1200 \mathrm{~g}$ at $4^{0} \mathrm{C}$ for 1 hour, during which neutralization took place. The $\mathrm{pH}$ of this neutralized extract was 
about 5 .

Instrumentation

The HPLC system used was purchased from Varian (Wallnut Creek, USA). It consisted of a liquid chromatograph Vista 5000 with a ultraviolet detector set at $254 \mathrm{~nm}$ (UV-100), in combination to a datasystem (Model 604) and a data print-out device.

A reversed-phase analytical column with a length of $250 \mathrm{~mm}$, an internal diameter of 4 $\mathrm{mm}$, and packed with spherical lichrosorb RP-18 particles of $4 \mu \mathrm{m}$ (Merck, Darmstadt, FRG) was used.

Mobile phase

A gradient system with two solvents was applied. Solvent A contained $20 \mathrm{ml}$ phosphoric acid $85 \%$ in $800 \mathrm{ml}$ distilled water. The pH of this solution was adjusted to 4.00 with $\mathrm{NH}_{4} \mathrm{OH}$ ( $25 \%$, in water). Water was added to obtain a final volume of $1000 \mathrm{ml}$. Solvent B consisted of acetonitrile and methanol ( $1 / 1$, by volume). Both mobile phase solvents were filtered through a $5 \mu \mathrm{m}$ filter (Sartorius, Gotingen, FRG). The chemicals to prepare the mobile phase solvents were purchased from Merck (Darmstadt, FRG).

During the first $5 \mathrm{~min}$ the elution run was kept isocratic with $100 \%$ solvent $\mathrm{A}$. Then a linear gradient was started from $100 \%$ solvent $\mathrm{A}$ at 5 min to $85 \%$ solvent $\mathrm{A}$ and $15 \%$ solvent $B$ at $15 \mathrm{~min}$. In the next five minutes the mobile phase was restored to $100 \%$ solvent A. A new sample was introduced following 15 minutes re-equilibration at $100 \%$ solvent A. The flow was kept constant during the whole run at $0.7 \mathrm{ml} / \mathrm{min}$.

The present method was based on a procedure described by Wynants and Van Belle (Wynants,1985), but was extended and modified to meet the requirements of our study on ischemic damage in kidneys. To this end the nature of the solid phase of the column and the $\mathrm{pH}$ of the eluent were changed. This resulted in better separation, less peak overlap, more components to be determined (from 15 to 19), a simplified gradient elution scheme, an increased life-time of the column (from 250 to 600 runs), and a shorter run time.

Detection limits

Detection limits were determined as described by Scott et al. (Scott,1977) Determination of the detector noise (Nd) was performed by measuring the maximum amplitude of the short and long term noise for a period of $10 \mathrm{~min}$ in $\mathrm{mV}$. The detector response to each compound was assessed and expressed as $R c=(h . w . Q) / m$

where $\mathrm{Rc}$ is detector response, $\mathrm{h}$ is peak height in $\mathrm{mV}$, w is peak width in min at a peak height of 0.607 of the maximum peak height, $Q$ is the flow rate of the mobile phase in $\mathrm{ml} / \mathrm{min}$, and $\mathrm{m}$ is mass in $\mathrm{g}$ of the injected solute. When peaks are symmetrical, w is independent of the solute concentration. In that case Rc can be expressed as

$\mathrm{Rc}=(\mathrm{c} / \mathrm{m}) \cdot \mathrm{h}$

introducing $\mathrm{c}$ as a constant factor. A signal to noise ratio of 2 was accepted to permit unambiguous identification of a signal. Hence the detection limit (DL) can be expressed as $\mathrm{DL}=\mathrm{c} \cdot(2 \mathrm{Nd} / \mathrm{m})$

Reagents

All chemicals used were of the highest purity available. GTP, GDP, GMP, IMP, ADP, and AMP were purchased from Boehringer (Mannheim, FRG). Hypoxanthine and adenosine were obtained from Merck (Darmstadt, FRG). ATP, inosine, xanthine, NAD, NADP, coenzyme A, uric acid, xanthosine, guanosine, adenylic succinate, and XMP were obtained from Sigma (St. Lowis, USA). 


\section{RESULTS}

Separation of purine metabolites

The present analytical method allows separation of 19 purine metabolites in a standard solution using a gradient HPLC system (figure 8.1). The substances tested were ATP, ADP, AMP, GTP, GDP, GMP, IMP, adenylic succinate, adenosine, inosine, hypoxanthine, xanthine, xanthosine, XMP, guanosine, uric acid, coenzyme $A, N A D$ and NADPH. Duration of a single run was about $25 \mathrm{~min}$.

Methodological variability

The within-day coefficient of variance was estimated by repeated determinations of the standard mixture on the same day. The results of twelve consecutive analyses showed

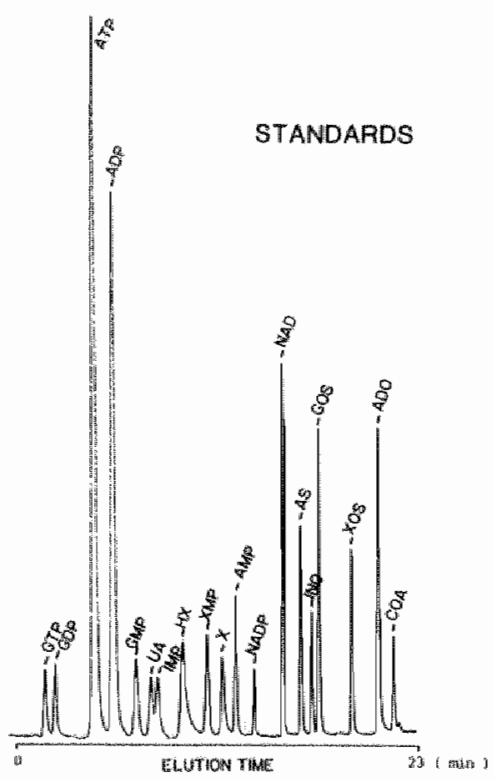

Figure 8.1: Chromatogram of a standard solution containing 19 purine compounds. Injection volume, $30 \mathrm{mil}$; wravelength $254 \mathrm{~nm}$. INO, inosine; Ado, adenosine; HX, hypoxanthine; $X_{\text {, xanthine; }}$, $S$, adenylic succinate: $X O S$, xanthosine; GOS guanosine; UA, uric acid.

that the within day or intra-assay coefficient of variance ranged from $1.4 \%$ for NAD to 4.3\% for IMP (table 8.Ia).

The between-day or inter-assay coefficient of variance was assessed by determining the content of the purine metabolites in the standard mixture on 11 consecutive days. The average inter-assay coefficient of variance for all substances was found to be about $5 \%$. The highest value was found for XMP i.e. 9.2\% (table 8.Ia).

Detection level and linearity

The content of purine metabolites in human kidney

Table 8.3 shows the values of ATP, ADP, AMP, GTP, GDP, GMP, IMP, inosine, adenosine, hypoxanthine, and xanthine in biopsy-specimen, obtained with a surgical blade in 


\begin{tabular}{|c|c|c|c|c|c|c|c|c|c|c|c|c|c|c|c|c|}
\hline & $\mathrm{APP}$ & $A D P$ & AMP & GTP & $\mathrm{GDP}$ & GMP & IMP & AS & INO & ADO & $\mathrm{k}: \mathrm{X} X$ & $\mathrm{x}$ & Kos & 605 & XMP & a \\
\hline $\begin{array}{l}\text { Mubut in- } \\
\text { bected or }\end{array}$ & & & & & & & & & & & & & & & & \\
\hline collumin & 253.5 & 1793 & 281 & 244 & 237 & 260 & 323 & $46 ?$ & 314 & 633 & 850 & 53 है & 553 & 634 & 531 & 211 \\
\hline Intra-assin & 1.7 & 2,1 & 2.9 & 2.5 & 2.8 & 1.9 & 4.3 & 1. 6 & 2.2 & 2.9 & 2.8 & 2.1 & 1.6 & 1.9 & $2+5$ & 3.7 \\
\hline Intem & 1.6 & 2.5 & 4.3 & 1.9 & 3.5 & 8. 4 & T. 1 & 5.3 & 3.8 & 3.9 & 4.0 & 7.2 & 17.1 & 6.2 & $9 \cdot 2$ & $6+3$ \\
\hline
\end{tabular}

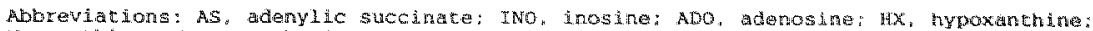

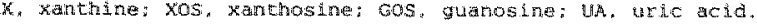

human kidney $(\mathrm{N}=11)$. NAD, NADP, and COA, as well as purine metabolites below the level of detection, were not listed. The kidneys investigated were not affected by ischemia.

Intra-individual and inter-individual coefficients of variance.

The renal tissue content of purine metabolites during transplantation.

In figure 8.2 the chromatograms of metabolites in tissue-specimen obtained during donor nephrectomy (left panel), at the end of 48 hours cold storage (middle panel), and one hour after reinstallment of the blood flow (right panel) are shown. In these repre-

\begin{tabular}{|c|c|c|c|c|c|c|c|c|c|c|c|c|}
\hline & ATP & $A D P$ & AMP & AN & GTP & GDP & GMP & $\mathrm{IMP}$ & INO & $\mathrm{ADO}$ & $\operatorname{six}$ & $\mathrm{x}$ \\
\hline Intralndsidual & 9.2 & 9.1 & 5.9 & 6.8 & 1.8 & 5.0 & 8.2 & 8.2 & 4.7 & 8.3 & 6.0 & 8.9 \\
\hline Intexind1vldua. & 25 & $1 \mathrm{~g}$ & 42 & 12 & 7 & 12 & 47 & 75 & 100 & 71 & 61 & $\ldots$ \\
\hline
\end{tabular}

AM, 1.e. sum of ATP, A.DP, and AMP; further abbreviations as in ifigure a.1.

sentative examples, it is demonstrated that the total adenine nucleotide pool, i.e. the sum of ATP, ADP, and AMP fell to 55\% of normoxic control values during 48 hours of cold

\begin{tabular}{|c|c|c|c|c|c|c|c|c|c|c|c|}
\hline & ATP & $A D B$ & Awp & Gre & $\mathrm{CDO}^{x}$ & $\operatorname{Gen} P^{x}$ & $M M P$ & THO & $\triangle D O$ & $\mathrm{Mx}$ & $x$ \\
\hline 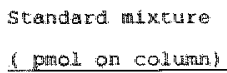 & 32 & 34 & 18 & 33 & 29 & 30 & 54 & 22 & 19 & 107 & 415 \\
\hline
\end{tabular}


storage in Eurocollins solution (middle pamel). Concomitantly, significant amounts of degradation products like hypoxanthine, xanthine, inosine, and IMP were found to accumulate in the stored kidney. One hour after reperfusion of the transplanted kidney the total adenine nucleotide pool did not increase. However, the energy charge ( (ATP + 1/2 ADP ) /( ATP + ADP + AMP ) according to Atkinson (Atkinson,1968), re-

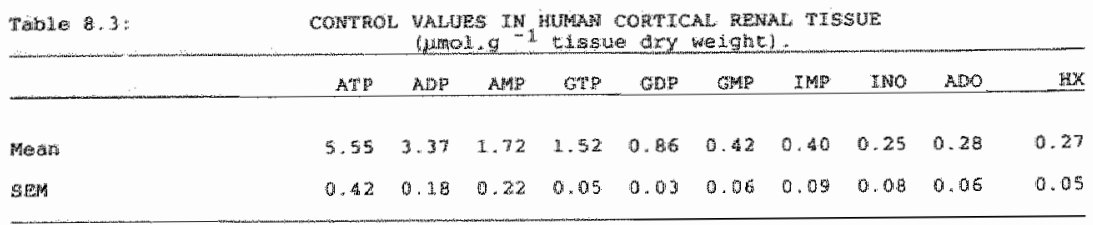

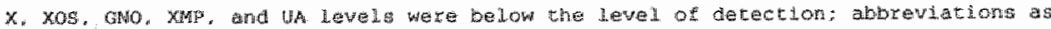
Hin tingure 2.1 .

covered completely in the reperfused transplanted organ ( $0.64 \mathrm{vs.} 0.38$ in the cold stored kidney, whereas the control value was found to be 0.64 ). Virtually all degradation products disappeared from the reperfused renal tissue (left panel).

\section{DISCUSSION}

The present report describes a sensitive HPLC system to monitor a variety of purine metabolites in human kidney tissue specimen. This technique is based on an assay procedure developed by Wynants and Van Belle (Wynants, 1985), which has been substantially modified, extended and evaluated to be applicable in the clinical setting. The need for such a technique is increasing as determination of adenine nucleotides and rellated purine substances has been suggested to be a powerful tool to assess the extent of ischemia-elicited damage to kidneys in renal surgery and renal transplantation. With such a tool ischemia time can be estimated as well as the effect of drug administration to counteract ischemia and reperfusion damage, and the efficacy of cold storage methods for preservation of donor organs. A possible drawback of the present technique is the invasive nature of the biopsy procedure (Takenaka,1980;Buhl,1979). However, biopsies are already routinely taken from transplantation kidneys for histological purposes without any major harm for the organ. In addition, the amount of tissue required for the subsequent HPLC analysis is very small i.e. in the order of $5 \mathrm{mg}$ of tissue wet weight. The advantage of the HPLC technique over non-invasive techniques like NMR is the fact that the whole spectrum of purines can be monitored. Similarly, with bioluminescence assays only ATP levels can be measured (White,1987). The intra and inter-assay coefficient of variance remained within acceptable limits. The intra-individual coefficient of variance was small, indicating a homogeneous distribution of the purine substances in the cortex of human kidneys. The inter-individual variability was, however, rather large. As has been pointed out elsewhere (Maessen,1988a) the surgical blade technique which is used to obtain kidney biopsies within the clinical setting results in increased AMP levels at the expense of ATP. This is due to the fact that a short period of non-perfusion of the tissue to be removed is created. The time duration of this procedure lies in between 15 to 30 sec. Previous studies in dog have shown that this time interval is large enough to induce a shift within the adenine nucleotide spectrum in renal tissue. It should 


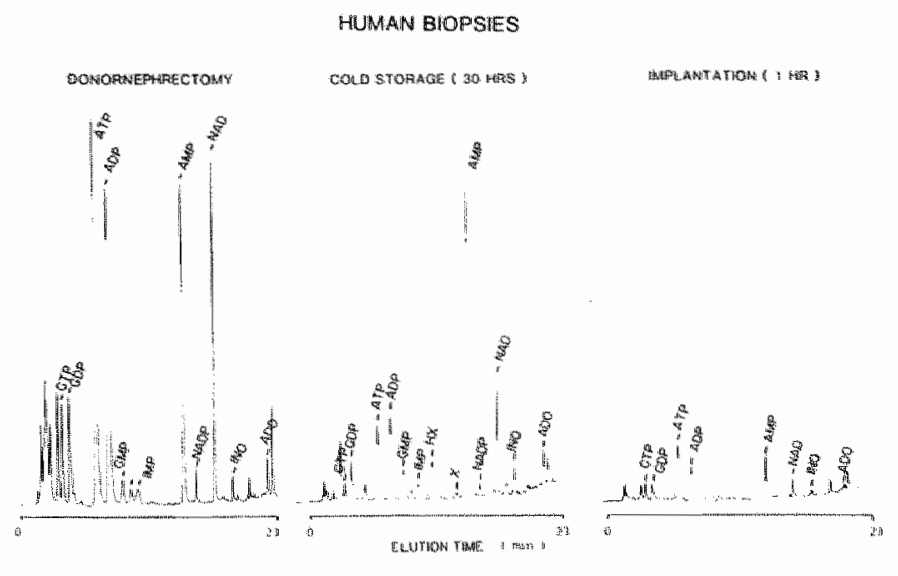

Chromatograms of human biopsies from one kidney in several conditions. Abbreviations as in figure 8.1. The biopsies in left, middlle, and right panel were weighing $7.3,3.2$, and $1.1 \mathrm{mg}$ tissue dry weight, respectively. It should be noted that the proportional difference between the chromatograms is the result of differences in biopsy weight. The sum of ATP, ADP, and AMP was $12.2,7.1$, and $6.4 \mu \mathrm{mol} / \mathrm{g}$ tissue dry weight before cold storage, at the end of 30 hours cold storage, and following one hour reperfusion, respectively. The corresponding values of the sum of the degradation products IMP, inosine, adenosine, hypoxanthine, and xanthine were $1.1,2.9$, and $0.3 \mu \mathrm{mol} / \mathrm{g}$ tissue $\mathrm{dry}$ weight, respectively.

be stressed that the sum of ATP, ADP, and AMP remains unaltered and shows a small inter-individual variability. No degradation products like purine bases and oxypurines are produced during the time interval required to obtain the tissue specimen. ATP levels per se are of minor value for monitoring of ischemia time since ATP levels rapidly decline in ischemic renal tissue, reaching nadir levels within 15 to $30 \mathrm{~min}$ of normothermic ischemia (Pegg, 1985;Buhl,1979). In summary, an experimental HPLC technique has been described for the purpose of monitoring the extent of ischemic injury in kidneys during renal surgery and renal transplantation. In addition to nucleotides as ATP, ADP, AMP, GTP, GDP, GMP, and IMP, related catabolic products like adenylic succinate, adenosine, inosine, hypoxanthine, xanthine, xanthosine, guanosine, XMP, and uric acid can be monitored in a single HPLC run. Tissue specimen with a weight of $5 \mathrm{mg}$ tissue wet weight are large enough to perform a determination of the spectrum of the most relevant purine metabolites.

\section{SUMMARY}

An HPLC technique is presented to determine tissue levels of adenine nucleotides and related substances in human kidneys. Effective separation of 19 metabolic substances is obtained in one single run of 25 minutes with agradient elution system. The mean intraassay coefficient of variance is $2.4 \%$, the inter assay coefficient of variance $5 \%$. The lower detection limit of substances commonly present in kidney tissue such as ATP, ADP, AMP, GTP, GDP, GMP, IMP, inosine, adenosine, hypoxanthine, and xanthine, ranges 
from 0.6 to $3.6 \mu \mathrm{mol} / \mathrm{h}$ corresponding to 18 and $107 \mathrm{pmol}$ on column. For reliable analysis tissue specimen of at least $5 \mathrm{mg}$ wet weight from renal cortical tissue during donor nephrectomy, during cold storage, and one hour following the onset of reperfusion, can be used. The method presented provides a rapid and reproducible diagnostic tool to examine the chemical energy status of human kidneys in renal surgery and transplantation 


\section{CHAPTER 9}

\section{THE STUNNED KIDNEY: POSTISCHEMIC RENAL FAILURE AND ADENINE NUCLEOTIDE HOMEOSTASIS ${ }^{1}$}

\section{INTRODUCTION}

The mechanisms underlying the development of acute renal failure in kidneys subjected to transient ischemia are not elucidated. In recent years much attention has been paid to the possible involvement of disturbed cellular adenine nucleotide homeostasis in ischemia-elicited damage of the kidney. In general, induction of a oxygen deficit by lack of proper blood supply causes the intracellular high energy phosphate store to be depleted with concomitant inhibition of membrane transport mechanisms. Following restoration of the blood flow the high energy phosphate pool is replenished and renal function may recover. When ischemia time is prolonged with ongoing degradation of high energy phosphates to nucleosides and purines, cells may become sooner or later unable to cover their basic energy requirements and die. Thus failed resynthesis of energy metabolites during reperfusion may lead to organ death (Chaudry, 1983). Although in some studies evidence has been gathered to confirm this basic concept (Gaudio,1983; Sinsteden,1986), other studies have failed to establish a relationship between changes of tissue levels of energy metabolites and postischemic functional correlates (Cooper,1986; Weinberg,1986). Therefore a direct causal relationship between the renal tissue content of adenine nucleotides and the ability of the kidney to exert adequate lifesustaining function is uncertain. Correct interpretation of the experimentally obtained data is hampered by the fact that generally the postischemic recovery of the adenine nucleotide store was only studied during early reperfusion. Furthermore, in most studies immediate contralateral nephrectomy was performed to monitor the concomitant functional recovery of the ischemic kidney. Lack of immediate life sustaining function will cause death of the experimental animal in the unilateral model but does not exclude recovery of the kidney at long term.

The present study was designed to obtain more insight in the relationship, if any, in renal adenine nucleotide content and restoration of life sustaining function. Changes of the cellular adenine nucleotide homeostasis were examined during moderate and severe ischemic damage and the time course of recovery during short term as well as long term reperfusion. Studies were performed in a canine model with renal artery obstruction during 30 and 90 minutes. The effect of ischemia on renal viability was assessed by determining the life sustaining function of the ischemic kidney following either immediate contralateral nephrectomy or delayed contralateral nephrectomy after one week or delayed contralateral nephrectomy after five weeks. Changes in tissue content of adenine nucleotides and their degradation products were measured in cortical biopsies with a HPLC technique. It was found that the impaired recovery of the cellular energy metabolism during postischemic reperfusion is only temporarily and that the energy metabolite levels after $90 \mathrm{~min}$ of ischemia are restored within $48 \mathrm{Hrs}$. Despite the obviously 
normalized energy metabolism long term renal failure developed in these kidneys (in the unilateral model). However the viability of these organs and the functional potency was maintained as after 5 weeks of delayed contralateral nephrectomy the kidneys appeared to have recovered to life sustaining function.

\section{MATERIALS AND METHODS}

General protocol

Experiments were performed on adult mongrel dogs of both sexes weighing between 18 and $25 \mathrm{~kg}$. Anesthesia was induced with thiopentotal ( $20 \mathrm{mg}$ per $\mathrm{kg}$ body weight) and maintained with a nitrous oxide-oxygen gas mixture, delivered continuously by a positive pressure respirator via an endotracheal tube. The animals were placed on a thermostatic watermattress. Kidneys were exposed through a midline abdominal incision. The left kidney was carefully dissected from its surrounding tissue. Ischemia was induced by cross-clamping the renal vessels. During the ischemic interval the abdomen was kept closed. In a number of groups the contralateral kidney was removed before release of the clamp. One hour after reperfusion of the ischemically damaged kidney, the abdomen was closed and the animals were allowed to recover.

Experimental groups

The dogs were allocated at random to six groups of experiments. In group I ( $n=3)$ and III $(n=6)$ the ischemic period lasted for 30 minutes. Contralateral nephrectomy was performed just prior to reinstallment of blood flow in the ischemic kidney. Dogs were allowed to recover for 2 and 5 days in group $I$ and $I$, respectively. The animals were sacrificed at the end of these intervals with an overdoses of thiopentotal. In group III ( $\mathrm{n}=$ 3 ) and IV $(n=6)$ the kidney was rendered ischemic for 90 minutes. Contralateral nephrectomy was performed just prior to reinstallment of the blood flow in the ischemic kidney. Dogs were allowed to recover for 2 and 5 days in group III and IV, respectively. In group V $(n=6)$ and VI $(n=5)$ the left kidney was made ischemic for 90 minutes. Contralateral nephrectomy was performed 7 and 35 days after reinstallment of the blood flow in group V and VI, respectively. In group I through VI two aspects were studied. First, the effect of ischemia and reperfusion on the adenine nucleotide homeostasis of the affected organ was examined. Wedge-shaped biopsies were taken with a surgical knife from the renal cortex. These tissue specimen, weighing in the order of $40 \mathrm{mg}$ wet weight, were taken prior to occlusion of the renal artery at the end of the ischemic period, and at 5 and 60 minutes following the release of the vascular clamp (group I to IV). Additional biopsies were taken at the day the animals were sacrificed (group I to VI). Second, the effect of ischemia and reperfusion on the life sustaining function of the affected kidney was studied. The life sustaining function was assessed as the capability of the animal to survive the renal ischemic insult. In addition, serum creatinine levels were determined daily in support to the clinical observation. Animals in group II, IV, and V were sacrificed at day 5 . They were considered non-survivors when they were clinically moribund and their serum creatinine levels kept rising, as in pilot experiments it had been found that after 90 minutes of ischemia and immediate contralateral nephrectomy animals died with severe symptoms of azotemia and continuously rising levels of serum creatinine within 5 to six days. Animals were considered survivors when their serum creatinine levels stabilized or decreased between day 4 and 5. Analysis of energy metabolites 
All cortical tissue samples were stored at $-80^{\circ} \mathrm{C}$. Before analysis the tissue samples were lyophilized at $-30^{\circ} \mathrm{C}$. Adherent blood was removed. The samples were weighed and extracted in $25-50 \mu \mathrm{L} / \mathrm{mg}$ tissue HCLO4 ( $2 \mathrm{~mol} / \mathrm{L}$ ), containing $5 \mathrm{mM}$ dithiothreitol. The tissue extract-HCLO 4 mixture was centrifuged at $1200 \mathrm{~g}$ for 5 minutes at $4^{\circ} \mathrm{C}$ and the supernatant was frozen in liquid nitrogen. Following addition of $40-80 \mu \mathrm{L} / \mathrm{mg}$ tissue $\mathrm{KHCO}_{3}(2 \mathrm{~mol} / \mathrm{L}$ ) to the supernatant, this mixture was allowed to thaw during centrifugation at $1200 \mathrm{~g}$ at $4^{\circ} \mathrm{C}$ for 1 hour, during which neutralization took place. Analysis of the samples was performed by a slightly modified version of the HPLC technique described by Wynants and wan Belle (1985). A Varian vista 5000 (Varian, Wallnut Creek, California) equipped with a narrow bore, stainless steel column filled with spherical liChrosorb RP-18 particles of $5 \mu \mathrm{m}$ (Merck,Darmstadt,FRG) was used for analysis. Injection volume of standard or sample extract was $10 \mu \mathrm{L}$. A gradient system was applied. Solvent A contained $0.15 \mathrm{M} \mathrm{NH}_{4} \mathrm{H}_{2} \mathrm{PO}_{4}$ in water buffered to $\mathrm{pH} 6.0$ with $\mathrm{NH} 4 \mathrm{OH}$. A mixture of acetonitrile and methanol ( $1 / 1$, by volume) was used as solvent $B$. Elution was kept isocratic with solvent A during the first seven minutes.

Thereafter a gradient was installed from $100 \%$ solvent $A$ to $85 \%$ solvent $A$ during 23 minutes. The flow rate was linearly increased from 0.7 to $0.8 \mathrm{ml} / \mathrm{min}$ within the first 3 minutes and kept at $0.8 \mathrm{ml} / \mathrm{min}$ during the remaining 27 minutes of the run. After each single run the column was re-equilibrated during 15 minutes with $100 \%$ solvent $\mathrm{A}$. All chemicals were obtained from Merck. Peaks were quantitated at $254 \mathrm{~nm}$ with a Varian 604 datasystem using the conversion factor of peak area per concentration of a known external standard. This technique offered an effective separation of ATP, ADP, AMP, IMP, adenosine, inosine, hypoxanthine, and xanthine, present in extracts of renal cortical biopsies. The method had an day by day variation of $5 \%$ and a intra-assay variation less than $4 \%$ for the individual substances measured. All values were calculated in micromoles per gram of tissue dry weight. Standardization was repeated every 10th sample. Peak identification was performed by injection of known standards individually and in combination. Standards were of the highest purity available. ADP, AMP, and IMP were purchased from Boehringer (Mannheim, FRG), hypoxanthine and adenosine from Merck and ATP, inosine and xanthine from Sigma (St.Louis, USA)

Serum creatinine

Serum creatinine levels were determined with a Beckman creatinine analyzer using a Beckman creatinine testkit.

Data analysis

All values reported are given as mean \pm standard error. Values within one group were compared at the various intervals by Wilcoxon rank-sum test. Significance was assessed by a p less than 0.05 . Differences between groups were tested by the Mann-Whitney U test. Significance for this test was assessed by a p less than 0.05 . Total adenine nucleotide content was determined by summing ATP, ADP, and AMP. Similarly total degradation products were determined by summing IMP, inosine, adenosine, hypoxanthine, and xanthine.

\section{RESULTS}

Life sustaining function

Thirty minutes of ischemia (group I and II ) did not affect the life sustaining function of the ischemic kidney in a unilateral kidney model. Maximum serum creatinine levels were 
reached at day two after the ischemic insult ( see table 9.1). In group III and IV ( $90 \mathrm{~min}$ ischemia and immediate contralateral nephrectomy) all animals developed severe symptoms of azotemia. Serum creatinine levels dramatically increased within 24 hours and continued to rise until the animals were sacrificed. One animal in this group survived with a maximum serum creatinine level of $800 \mathrm{mmol} / \mathrm{l}$ at day 4 . Delayed contralateral nephrectomy at one week after the ischemic insult ( group V) did not improve survival. Serum creatinine levels at 5 days after nephrectomy were not significantly lower than corresponding values in group IV. Only one dog survived. If contralateral nephrectomy was postponed to 5 weeks after the ischemic insult (group VI) all kidneys showed de-

\begin{tabular}{|c|c|c|c|c|c|}
\hline Gxoup & 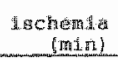 & tine & $\begin{array}{l}\text { contralatered. } \\
\text { rephroctionf }\end{array}$ & surew wors 1 & 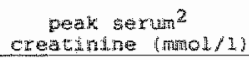 \\
\hline$I X$ & 30 & & imonealate & $5 / 6$ & $120-2.60$ \\
\hline $\mathrm{TV}$ & 90 & & Lunedsate: & $1 * 6$ & $800-1200$ \\
\hline v & 90 & & $\begin{array}{l}\text { dofayed: } \\
(7 \text { dans })\end{array}$ & $1 / 6$ & $800-1100$ \\
\hline VI & 90 & & $\begin{array}{l}\text { datayed } \\
\text { (35 days }\end{array}$ & $5 / 5$ & $130-300$ \\
\hline
\end{tabular}

\footnotetext{
1 See materdas and methods section for defindion survivors and non-gurvivors.

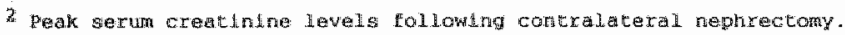

finitive recovery to life sustaining function ( animals were sacrificed in good health after 5 months ). Serum creatinine levels ranged from 130 to $300 \mathrm{mmol} / \mathrm{l}$ at day 5 following nephrectomy.

Adenine nucleotide homeostasis

The ischemic tissue adenine nucleotide content fell to 53 and $35 \%$ of control in response to 30 and 90 minutes of ischemia, respectively. The monophosphates (AMP and IMP) showed a transient increase during ischemia as after $30 \mathrm{~min}$ of ischemia these levels were higher than after $90 \mathrm{~min}$. AMP and IMP levels were still significantly higher after $90 \mathrm{~min}$ than control levels. ADP and ATP levels were significantly reduced after 30 minutes and continued to decrease when ischemia was extended to 90 minutes $(p<0.05)$. Inosine levels significantly increased during 30 and 90 minutes of ischemia. No significant changes of adenosine values were observed. The total increase of nucleosides, i.e. the sum of adenosine and inosine, remained less than $9 \%$ and $3 \%$ of the loss of nucleotides after 30 and 90 minutes, respectively. A profound accumulation of hypoxanthine according to the length of ischemia time was observed. Xanthine accumulated to a lesser extent than hypoxanthine in renal cortical tissue rendered ischemic for 30 and 90 minutes of ischemia ( table 9.2).

Reperfusion

The content of ATP and ADP increased in biopsies obtained from renal cortex reper- 


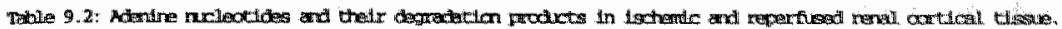
unit. $\left.\right|^{-1}$ ery wetge).

\begin{tabular}{|c|c|c|c|c|c|c|c|c|c|}
\hline & mas & AIP & $A C F$ & Ap & $\mathbb{P}$ & $\mathrm{TNO}$ & .100 & $\mathbb{W}$ & $\mathrm{x}$ \\
\hline $\begin{array}{l}\text { predstemic } \\
\text { ipdentic }\end{array}$ & $\pi$ & $4.0 \pm 0.3$ & $4.5 \pm 0.6$ & $3.4 \pm 0.5$ & $0.4 \pm 0.2$ & $0.2 \pm 0.1$ & $0.3 \pm 0.1$ & $\mathbb{1}=0.2$ & $0.2 \pm 0.2$ \\
\hline $\begin{array}{l}30 \text { min } \\
\text { renter funton }\end{array}$ & III & $0.8 \div 0.1 \mathrm{~m}$ & $1.3 \pm 0.1^{*}$ & $4.3 \pm 0.3$ & $1.0 \pm 0.1^{*}$ & $0.7 \pm 0.1 *$ & $0.2+0.0$ & $5_{n} 0: 0.3^{m}$ & $0.9 \pm 0.2$ \\
\hline 5 mitn & II & $2.3 \neq 0.3$ & $2.5 \neq 0.1$ & $2.5 \pm 0.4$ & $0.2 \pm 0.1$ & $0.1 \pm 0.0$ & $0.2 * 0.0$ & 1. 10.5 & $0.6 \pm 0.2$ \\
\hline 60 mintn & II & $3.8 \pm 0.4$ & $3.5 \pm 0.5$ & $2.3 \neq 0.3$ & $0.2 \pm 0.0$ & $0.1 \pm 0.0$ & $0.2 \pm 10.0$ & $1, z+0,3$ & $1.0 \pm 0.3$ \\
\hline 2 dexs: & $I$ & $5.9 \div 0.9$ & $3.9 \pm 0.6$ & $2.0 \pm 0.1$ & $0.4 \pm 0.2$ & $0.1 \pm 0.0$ & $0.1 \pm 0.0$ & $1.5 * 0.3$ & $0,4 \pm 0,1$ \\
\hline 5 days: & $\mathrm{II}$ & $5.8 \pm 1.5$ & $3.3 \pm 0.3$ & $2.0 \pm 0.6$ & $0.2 \pm 0.1$ & $0.1 \pm 0.0$ & $0.2 * 0.1$ & 1.0 出 0.2 & $0.2 \pm 0.1$ \\
\hline laschentic & & & & & & & & & \\
\hline $90 \mathrm{mtn}$ & $\pi$ & $0.4 \pm 0.0^{*}$ & $0.8+0.1 *$ & $3.0 \neq 0.5$ & $0.7 \pm 0.1$ & $0.5 \pm 0.1$ & $0.2 \pm 0.0$ & $7.9 \pm 0.7 *$ & $1.9 \pm 0.3$ \\
\hline$s \mathrm{~min}$ & IW & $1.1 \pm 0.1 \pm$ & $1.4 \pm 0.2^{* * *}$ & $14 \pm 0.2^{*}$ & $0.4 \pm 0.1$ & $0.1 \pm 0.1$ & $0.1 \neq 0.0$ & $11 . \pm 0.3$ & $0.6 \div 0.1$ \\
\hline $60 \mathrm{~min}$ & $\mathbb{W}$ & $2.3 \pm 0.5 * *$ & $1.9 \pm 0.2^{+4}$ & $1.5 \pm 0.2 * *$ & $0.1 \pm 0.0$ & $0.2 \pm 0.0$ & $0.1 \neq 0.0$ & $0 . \pm 0.2$ & $0.8 \times 0.2$ \\
\hline 2 ders & IIII & $4.16 \pm 0.5$ & $2.8 \pm 0.3$ & $1.9 \pm 0.2$ & $0.9 \pm 0.0$ & $0.3 \pm 0.0$ & $0.0 \pm 0.0$ & $0.5 \pm 0.5$ & $0.7 \neq 0.1$ \\
\hline 5 derss & IN & $5.7 \div 0.16$ & $2.9 \pm 0.4$ & $1.4 \pm 0.4$ & $0.1 * 0.0$ & $0.2 \neq 0.0$ & $0.0 \neq 0.0$ & $0.6 \pm 0.3$. & $0.5 \neq 0.2$ \\
\hline
\end{tabular}

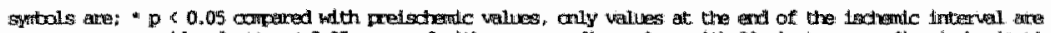

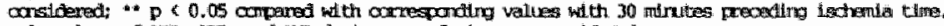

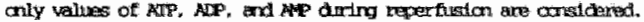

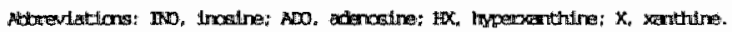

fused for 5 and 60 minutes following 30 minutes of ischemia ( table 9.2). AMP levels fell. The sum of ATP, ADP, and AMP was about $80 \%$ of the pre-ischemic value (figure 9.1 ) at 60 minutes of reperfusion. The increase of ATP and ADP together during this time interval (about $5.2 \mu \mathrm{mol} / \mathrm{g} \mathrm{dw}$ ) significantly exceeded the fall in AMP (about $2.0 \mu \mathrm{mol} / \mathrm{g}$ $\mathrm{dw}$ ). Although a comparable pattern was found in biopsies obtained from kidneys rendered ischemic for 90 minutes, the recovery was quantitatively less marked. ( table 9.2 and figure 9.1). After 60 minutes of reperfusion the increase in the content of ATP and ADP together amounted to about $3.0 \mu \mathrm{mol} / \mathrm{g} \mathrm{dw}$ with a concomitant fall in AMP content of $1.5 \mu \mathrm{mol} / \mathrm{g} \mathrm{dw}$. Figure 9.1 shows that the sum of ATP, ADP, and AMP reached values of about $47 \%$ of pre-ischemic control at 60 minutes of reperfusion following 90 minutes of ischemia..

Prolonged reperfusion for 2 and 5 days resulted in complete recovery of the tissue adenine nucleotide content in kidneys rendered ischemic for 30 minutes ( figure 9.1, group I and II ). The content of adenine nucleotides in kidneys subjected to ischemia for 90 minutes, returned almost completely to pre-ischemic control values after 2 and 5 days of reperfusion (figure 9.1, groups III and IV ). Differences between group I and III, and between II and IV did not reach the level of significance $(p<0.05)$. Renal adenine nucleotide levels in group V and VI measured before the animals were sacrificed, were similar to control (data not shown).

Within 5 minutes after reperfusion the tissue content of degradation products such as inosine, hypoxanthine, and xanthine was reduced to pre-ischemic values and remained at that level thereafter or further decreased ( table 9.2), irrespective of the duration of 


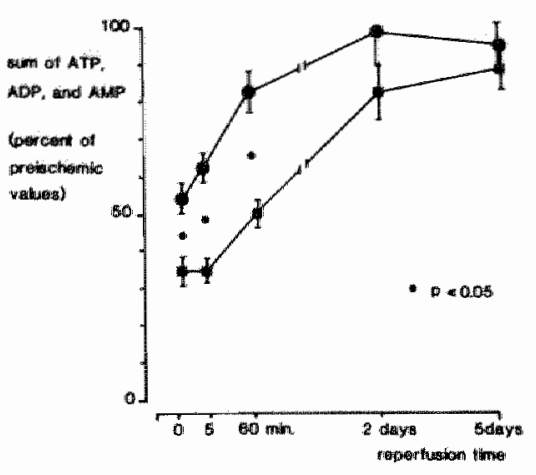

Figure 9.1.: Recovery of the content of adenine nucleotides in reperfused kidneys following 30 or 90 minutes of ischemia, respectively. The content of adenine nucleotides (sum of ATP, ADP, and AMP) is expressed as the percentage of pre-ischemic control values. Symbols are: circle, 30 minutes of preceding ischemia; rectangle, 90 minutes of preceding ischemia.

the preceding ischemic episode.

\section{DISCUSSION}

In the present study it is demonstrated that the impaired replenishment of the high energy nucleotide store after $90 \mathrm{~min}$ of ischemia is only transient as within $48 \mathrm{Hrs}$ the level of nucleotides has almost completely returned to normal. At this time no significant difference is found in nucleotide levels after 30 and 90 min of ischemia. In contrast, a marked and persistent difference is found in function in these groups. In the unilateral model 5 out of 6 dogs died within 5 days following $90 \mathrm{~min}$ of ischemia whereas after $30 \mathrm{~min}$ all animals survived.

The delayed recovery of the nucleotide pool after $90 \mathrm{~min}$ of ischemia early during reperfusion might be explained by the lower level of nucleotides at the end of the ischemic interval in comparison to this value after $30 \mathrm{~min}$ of ischemia. In studies in rabbit kidneys it was found that the rate of adenine nucleotide resynthesis upon reperfusion was independent to the level of depletion during ischemia (Buhl,1976). Hence, a lower level of adenine nucleotides after 90 minutes of ischemia and 60 minutes reperfusion might be a simple result of lower levels of adenine nucleotides at the beginning of reperfusion. In the present study in dog however it is found that the rate of adenine nucleotide recovery is markedly slower after $90 \mathrm{~min}$ of ischemia than after 30 minutes of ischemia. Apparently, the effect of the length of ischemia time is not only demonstrated by the decrease of ademine nucleotide levels during ischemia but is also demonstrated during reperfu- 
sion by an impairment of the adenine nucleotide recovery rate.

A role for the locally accumulated degradation products to this difference in rate of nucleotide recovery could not be firmly established. Several pathways in which conversion of degradation products may add to the resynthesis of nucleotides have been suggested (Murray,1971). Little, if any, of the decrease in the level of degradation products that was found within $5 \mathrm{~min}$ of reperfusion in this study is likely to be directly involved in the resynthesis process since the decrease was similar after 30 min of ischemia as after 90 min whereas in the latter group hardly any increase of nucleotides was observed within this interval. Simple wash out by recirculation is the most obvious explanation for the disappearance of the degradation products. This explanation is further evidenced by studies on renal preservation using hypothermic continuous perfusion (Buhl,1977; Takenaka,1980). During perfusion of ischemic kidneys hypoxanthine and xanthine were recovered in the perfusate and hence lost for nucleotide resynthesis. The decrease of the potential precursors however did not negatively affect the viability of these kidneys (Belzer,1985). It is unknown whether circulating hypoxanthine and xanthine may still play a role in intracellular nucleotide resynthesis in vivo. Since the almost complete recovery of the energy stores after 2 days of reperfusion following 90 min of ischemia, a recovery still present at the time the animals were sacrificed with severe symptoms of azotemia, suggested that the renal tissue remained viable ( group III and IV). Additional experiments were performed. The contralateral kidney was not removed until 1 and 5 weeks after the onset of reperfusion in order to extend the time for the ischemic kidney to recover functionally (group V and VI). Viability of the kidneys was confirmed as after 5 weeks of delayed contrallateral nephrectomy the ischemic kidneys had recovered to such an extent that life sustaining function was possible. Thus a large discrepancy in time was found between metabolic and functional recovery of the postischemic kidneys. It is feasible that recovery of the cellular energy metabolism precedes the functional recovery of the cell as a whole. It remains, however, puzzling that the concentration of the high energy nucleotides rapidly regain pre-ischemic concentrations despite lack of functional recovery whereas in other studies a tight quantitative relationship has been demonstrated between cellular ATP content and renal cellular transport processes in healthy tissue (Tessitore, 1986). The present data therefore suggest that a ATP-deficit is not the cause of the persisting renal failure. Slow recovery of structural cellular components may be considered to contribute to the delay in recovery.

The temporarily relief of the functional load on the ischemic kidney as obtained by delayed contralateral nephrectomy is not necessarily a stimulus for functional recovery. In previous studies it has been shown that the function of ischemically damaged kidneys is indeed improved by the immediate removal of healthy contralateral kidneys ( Fin,1980 ). Similarly, the recovery of ischemically damaged renal transplants is inhibited by the presence of one or two normal native kidneys in rats (Cofman,1986). Other studies revealed that the increased functional load or the changed environmental conditions rather than the hypertrophic stimulation by removing the contralateral kidney induced the enhanced functional recovery of the ischemic kidney (Fried,1984). It may thus be surprising that in the present study the ischemic kidneys appeared to regain life sustaining function only by contralateral nephrectomy for 5 weeks. The reason for these obviously paradoxical findings may be related to the severity of the injury to the ischemic kidney which was only moderate in previous studies (Fried,1984; Cofman,1986; Fin,1980). In 
addition, the time interval after which the recovery of the kidney was monitored was only within hours following the ischemic insult. Furthermore the beneficial effect of the immediate removal of the contralateral kidney was expressed in improved functional parameters such as creatinine clearance and tubular re-absorbtion rather than in terms of viability as was done in the present study. It is therefore possible that in the present study the presence of the contralateral kidney depressed the function of the ischemic kidney to some extent on the one hand and prevented the animal from dying before the ischemic kidney had recovered to life sustaining function, on the other hand. Hence, an important conclusion from the present experiments is that the renal tissue after $90 \mathrm{~min}$ of ischemia remained viable as suggested from the status of the energy metabolism and was eventually able to recover life sustaining function. In summary, the data in this study indicate that following severe ischemic injury a period may be recognized after circulation has been restored, in which renal functional failure persists whereas the levels of high energy metabolites have been normalized suggesting potentially viable cells. During this condition which may be described as "stunned kidney" analogous to the "stunned myocardium" (Braunwald,1982) the eventual functional recovery will depend on more factors than solely the status of the cellular energy metabolism. Clinically the above mentioned experimental condition may be represented for instance by posttransplant renal non-function due to preservation damage or preexisting ischemic damage which is usually falsely defined as "acute tubular necrosis".

\section{SUMMARY}

The content of adenine nucleotides in cortical biopsies from canine kidneys fell from 12.0 to 6.3 and $4.1 \mu \mathrm{mol} / \mathrm{g} \mathrm{dw}$ with a concomitant increase in degradation products such as IMP, inosine, hypoxanthine and xanthine after 30 and 90 minutes of ischemia. Following 60 minutes reperfusion tissue adenine nucleotide levels returned to $80 \%$ of control in kidneys rendered ischemic for 30 minutes. In contrast initial recovery of adenine nucleotides was poor in kidneys subjected to 90 minutes of ischemia. Prolongation of reperfusion for 2 to 5 days resulted in almost complete normalization of tissue adenine nucleotides in kidneys made ischemic for 30 or 90 minutes. The life sustaining function of the ischemically damaged kidneys was tested by removing the contralateral kidney just prior to reinstallation of the blood flow. All animals survived 30 minutes of ischemia renal ischemia. In contrast, 5 out of 6 animals died in the 90 minutes ischemia group, despite normalized adenine nucleotide levels. Delay of contralateral nephrectomy revealed that $100 \%$ survival of the animals was obtained when the non-ischemic kidneys were removed 5 weeks following reperfusion. Our data support the concept that postischemic adenine nucleotide resynthesis is depressed according to the extent of ischemic injury. However, it is also shown that the adenine nucleotides may rapidly gain normal levels whereas recovery of renal function is initially absent and may only become apparent if the kidney is allowed to recover for several weeks. 


\section{BENEFICIAL EFFECTS OF INTERMEDIATE NORMOTHERMIC PERFUSION DURING COLD STORAGE OF ISCHEMICALLY INJURED KIDNEYS ${ }^{1}$}

\section{INTRODUCTION}

The use of ischemically damaged kidneys for transplantation is probably inevitable when the availability of non-ischemic donor organs will, as is expected, become limited (Kootstra,1984). Fortunately, kidneys can readily recover from extensive ischemic injury (Sheehan,1953), Current preservation methods however, seem less appropriate to prevent damage during the storage of ischemic donor kidneys. The deleterious effect of the combination of hypothermic storage and preexisting ischemic injury on posttransplant function has long been recognized but not yet satisfactorily attenuated by modification of preservation techniques (Johnson,1972).

Many attempts to achieve better preservation have focused on the increase of renal adenine nucleotide levels by changing the composition of preservation perfusates without consistent results either on the restoration of the energy metabolism or the functional recovery of the kidneys (Pegg,1984;Garvin,1985). A new approach to improve preservation techniques has been presented by the introduction of a brief period of perfusion with blood using either a donor dog or a heart-lung machine, halfway preservation time (Kootstra,1980). In combination with hypothermic continuous perfusion the intermediate normothermic perfusion allows to double the maximum preservation time of canine kidneys (Rijkmans,1984). Recently, the effectiveness of intermediate normothermic perfusion has been confirmed by others (Gaber,1987;Mayfield,1987). The mechanism underlying the beneficial effect has not yet been established.

The present experimental study in dog was conducted to examine two questions. First, is the concept of intermediate normothermic perfusion useful to improve the preservation properties of simple cold storage of ischemically injured kidneys, and second, what is the effect of cold storage on the energy metabolism in ischemic kidneys and how is it influenced by intermediate normothermic perfusion. Energy metabolism was studied by monitoring changes in the total pool of renal purine nucleotides including adenine nucleotides, guanine nucleotides and IMP, representing the high energy phosphates and their immediate precursors. The viability of kidneys was tested in a unilateral autologous transplantmodel.

\section{MATERIALS AND METHODS}

\section{General protocol}

Experiments were performed on adult mongrel dogs of both sexes weighing between 18 and $25 \mathrm{~kg}$. Anaesthesia was induced with thiopentotal ( $20 \mathrm{mg}$ per $\mathrm{kg}$ body weight) and maintained with a nitrous oxide-oxygen gas mixture, delivered continuously by a positive pressure respirator via an endotracheal tube. The animals were fasted during 24 hours before surgery with free access to water. During surgery body temperature was 
kept constant using a thermostatic watermattress. Kidneys were exposed through a subcostal incision. The left kidney was carefully dissected from the surrounding tissue. Ischemia was induced by cross clamping the renal vessel pedicle. During the ischemic interval the abdomen was kept closed. Following removal of the left kidney, flushing with Eurocollins at $4^{\circ} \mathrm{C}$ from a bottle at a height of $180 \mathrm{~cm}$ was started within 3 minutes. Flushing was continued during 8 minutes. Kidneys were then wrapped in plastic bags and stored on ice. Following cold storage the kidneys were re-implanted autologously through a midline abdominal incision onto the iliac vessels. The ureter was implanted in the bladder. This procedure took 20 to 30 minutes and was performed by the same surgical team in each case. During this procedure additional ischemic injury may be expected. Immediate contralateral nephrectomy was performed before re-anastomosis was established. One hour after recirculation of the implanted kidney, the abdomen was closed and the animals were allowed to recover. When the animals survived up to 14 days, they were considered as survivors.

\section{Intermediate normothermic perfusion}

Some kidneys were halfway during their preservation time, i.e. after 24 hours, perfused for two and a half hour by temporarily connection to the circulation of the donor dog. Under sedation the left carotid artery and left femoral vein of the dog was cannulated using polyethylene tubing. The kidney was placed in a dispasable organ chamber in which the temperature was kept constant at $38^{\circ} \mathrm{C}$. To start the perfusion the arterial line was connected to a cannula in the renal artery. The arterial connection was kept as short as possible $(8-10 \mathrm{~cm})$. The venous effluent was collected in the conically shaped bottom of the organ chamber and pumped back to the animal using a roller pump. A pressure transducer was mounted on the arterial line to measure the blood pressure to the kidney. At the end of the normothermic perfusion the kidneys were flushed with Eurocollins, wrapped in plastic bags, and again stored on ice for 24 hours. The catheters in the carotid artery and femoral vein were removed and the blood donor animals were allowed to recover.

\section{Experimental groups}

Dogs were allocated at random to 5 experimental groups. In group I and II kidneys were not subjected to ischemia and immediately removed upon dissection. They were stored on ice during 24 (group I) and 48 hours (group II). In Group III through V the vessel pedicle of the kidneys was cross-clamped during 30 minutes before removal to induce normothermic renal ischemia. After dissection kidneys were stored during 24 (group III) and 48 hours (group IV and V). In group V kidneys underwent a ex-vivo perfusion half way storage time (for procedure see above). All kidneys were re-implanted as described above. In each group 6 experiments were performed. This experimental design was chosen to study, first, the effect of preceding warm ischemia and the length of cold storage time on the nucleotide content of kidneys at the end of colld storage and following re-implantation, and second, the influence of a brief normothermic ex vivo perfusion during preservation on the nucleotide homeostasis in kidneys with preceding ischemic damage. Finally the life sustaining function was tested to study the effect of this procedure on renal viability.

The nucleotide content of the renal tissue was determined in renal tissue specimen, 
weighing about $40 \mathrm{mg}$ wet weight. To this end, wedge-shaped biopsies were taken with a surgical knife from the renal cortex at the end of the warm ischemic interval, at the end of the cold storage period, at 60 minutes of ex-vivo perfusion, and at 60 minutes of reperfusion following implantation. In these tissue specimen the concentration of ATP, ADP, AMP, GTP, GDP, GMP, and IMP was determined as well as the concentration of the major degradation products of these nucleotides including adenosine, inosine, hypoxanthine and xanthine.

\section{Analysis of energy metabolites}

The concentration of the energy metabolites was determined using a HPLC method as described previously (Maessen,1988). Briefly, all cortical tissue samples were lyophilized at $-30^{\circ} \mathrm{C}$. Adherent dried blood was subsequently removed. The samples were weighed and extracted in $25-50 \mu \mathrm{L} / \mathrm{mg}$ tissue $\mathrm{HCLO}_{4}(2 \mathrm{~mol} / \mathrm{L})$, containing $5 \mathrm{mmol} / /$ dithiothreitol. The tissue extract-HCLO ${ }_{4}$ mixture was centrifuged and the supernatant was frozen in liquid nitrogen. Following addition of $40-80 \mu \mathrm{L} \mathrm{KHCO}_{3}(2 \mathrm{~mol} / \mathrm{L})$ per $\mathrm{mg}$ tissue to the supernatant, this mixture was allowed to thaw during centrifugation at $1200 \mathrm{~g}$ at $4^{0} \mathrm{C}$ for 1 hour, during which neutralization took place.

A Varian Vista 5000 (Varian, Wallnut Creek, California) equiped with a narrow bore, stainless steel column filled with spherical lichrosorb RP-18 particles of $5 \mu \mathrm{m}$ (Merck,Darmstadt,FRG) was used for a gradient HPLC analysis. Injection volume of standard or sample extract was 10 microliter. After each single run which took 30 minutes the column was re-equilibrated for 15 minutes. All chemicals were obtained from Merck (Darmstadt,FRG). Peaks were quantitated at $254 \mathrm{~nm}$ with a Varian 604 datasystem using the conversion factor of peak area per concentration of a known external standard. This technique offered an effective separation of ATP, ADP, AMP, GTP, GDP, GMP, IMP, adenosine, inosine, hypoxanthine, and xanthine, present in extracts of renal cortical biopsies. The method had an day by day variation of $5 \%$ and a intra-assay variation less than $4 \%$ for the individual substances measured. All values were calculated in mmoles per gram of tissue dry weight. Standardization was repeated every 10th sample. Peak identification was performed by injection of known standards individually and in combination. Standards were of the highest purity available. ADP, AMP, GTP, GDP, GMP, and IMP were purchased from Boehringer (Mannheim, FRG), hypoxanthine and adenosine from Merck and ATP, inosine and xanthine from Sigma (St.Louis, USA).

\section{Data analysis}

All values reported are given as mean \pm standard error. Values within one group were compared at the various intervals by Wilcoxon rank-sum test. Significance was assessed by a p less than 0.05 . Differences between groups were tested by the Mann-Whitney $U$ test. Significance for this test was assessed by a p less than 0.05 . Total adenine nucleotide content was determined by summing ATP, ADP, and AMP. Similarly, total guanine nucleotide content is the sum of GTP, GDP, and GMP. The total nucleotide pool was considered the sum of the contents of the adenine nucleotides, the guanine nucleotides and IMP. The content of degradation products was determined by summing adenosine, inosine, hypoxanthine, and xanthine.

\section{RESULTS}


Posttransplant viability

In table 10.1 the viability of the kidneys in each experimental group as tested in a unilateral transplant model with immediate contralateral nephrectomy is shown. Twenty-four hours cold storage did not affect the viability of kidneys without warm ischemia. In all other groups the viability was $50 \%$ or less. No animals with a kidney subjected to 30 minutes of warm ischemia and 48 hours of cold storage survived. However, if the kidneys were ex-vivo perfused halfway during storage time a survival of 3 out of 6 was obtained.

Nucleotide homeostasis: cold storage

During 24 hours of cold storage the adenine nucleotide content of canine kidneys without preceding warm ischemia fell by about $30 \%$ (table 10.2). The content of guanine nucleotides also fell by $30 \%$. The content of IMP significantly increased. Thus, cold stor-

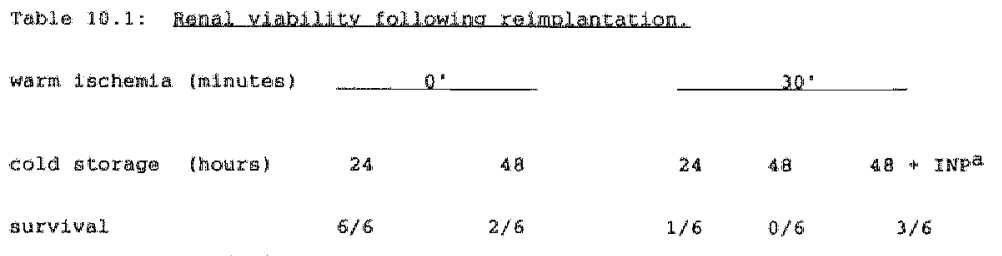

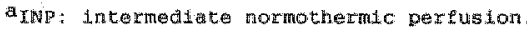

age reduced the total pool of tissue nucleotides significantly by about $3 \mu \mathrm{mol} / \mathrm{g} \mathrm{dw}$. Prolongation of storage time to 48 hours did neither further reduce the total pool of tissue nucleotides nor alter the balance between adenine nucleotides, guanine nucleotides, and IMP (table 10.2). If kidneys were subjected to 30 minutes of warm ischemia prior to cold storage the adenine and guanine nucleotide content had already fell before the start of cold preservation with a significant increase in the tissue content of IMP.

Cold storage during 24 hours of kidneys after 30 minutes of warm ischemia did not significantly affect the content of adenine and guanine nucleotides but IMP levels further increased. Prolongation of storage time to 48 hours resulted in a significant further decrease of the adenine and guanine nucleotide content by 2.4 and $0.4 \mu \mathrm{mol} / \mathrm{g} \mathrm{dw}$, respectively. The content of IMP did not show an additional increase. Hence, from the pre-ischemic total nucleotide pool of $12.0 \mu \mathrm{mol}, 6.7 \mu \mathrm{mol} / \mathrm{g} \mathrm{dw}$ was left after 30 minutes of warm ischemia time and 48 hours cold storage (table 10.2).

Intermediate normothermic perfusion

The loss of nucleotides during the preservation interval between 24 and 48 hours amounted to $3.2 \mu \mathrm{mol} / \mathrm{g} \mathrm{dw}$ without intermediate normothermic perfusion (figure 10.1). This loss was significantly reduced to $1.4 \mathrm{mmol} / \mathrm{g} \mathrm{dw}$ at the end of the same interval if the intermediate normothermic perfusion was applied after 24 hours of cold storage. 


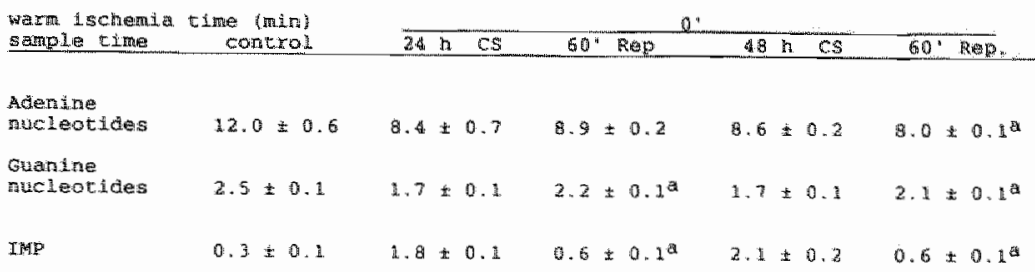

Table 10.2.b: Renal control nucleatide content following cold scorage and relmplantation.

\begin{tabular}{|c|c|c|c|c|c|c|c|c|c|c|}
\hline \multirow{2}{*}{$\begin{array}{l}\text { Warm ischernia time (min) } \\
\text { sample }\end{array}$} & \multicolumn{10}{|c|}{$-20^{\circ}$} \\
\hline & 30 & wit & $24 \mathrm{~b}$ & bn cs & $60^{\circ}$ & Rep & $40: 5$ & $\mathrm{Cs}$ & $60^{\circ}$ & Rep \\
\hline Actenlne mucleotides & 6.4 & $\pm_{0} 0.3$ & 6. 4. & \pm 0.3 & 5. & $\pm 0.1^{a}$ & 400 & 0.40 & 4. $y$ & 0.3 \\
\hline Guarine nucleotldes & 1.7 & \pm 0.0 & $1.6 \pm$ & \pm 0.0 & $1.9=$ & $\pm 0.0^{a t}$ & $1.2 i$ & $a, b^{b}$ & $1.5 x$ & 0.1 \\
\hline IMP & 1.0 & \pm 0.1 & $1.9 \pm$ & \pm 0.2 & $0.18=$ & $10.1^{a}$ & 1. $5 \pm$ & $=0.1$ & 0.0. & $0.1^{a}$ \\
\hline
\end{tabular}

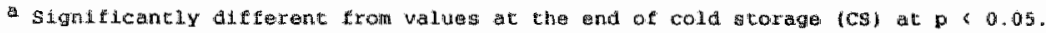

b signiflcanty different from values at 24 hours of cold atorage att p 0.05 .

Welles ate means $t$ SEM of 6 wperiments.

Abbrevations are: Cs, cold storage; hep. repereusion following implantations; will. Harm ischemila time.
}

The increased content of the nucleotide pool was due to the maintenance of higher adenine nucleotide levels ( $5.5 \pm 0.3$ vs. $4.0 \pm 0.4 \mu \mathrm{mol} / \mathrm{g} \mathrm{dw}$ ). Levels of guanine nucleotides and IMP were not significantly different $(1.1 \pm 0.2$ vs. $1.2 \pm 0.1$, and $1.9 \pm 0.2$ vs. $1.5 \pm 0.1 \mu \mathrm{mol} / \mathrm{g} \mathrm{dw}$, respectively). The direct effect of intermediate normothermic perfusion at $38^{0} \mathrm{C}$ on the tissue level of nucleotides and degradation products was studied. Biopsies were taken just prior to intermediate perfusion and after the first 60 minutes of the perfusion period. Results are given in table 10.3. Within the first 60 minutes of the total period of 2.5 hours of normothermic ex-vivo perfusion the total nucleotide pool, i.e. the sum of ATP, ADP, AMP, GTP, GDP, GMP, and IMP did not significantly change ( $9.9 \approx 0.3 \mathrm{vs} .9 .5 \pm 0.2 \mu \mathrm{mol} / \mathrm{g} \mathrm{dw}$ before the onset of reperfusion). The balance between the individual nucleotide levels, however, was greatly changed. The monophosphate content (AMP, GMP, and IMP) significantly decreased with a concomitant increase of di- and tri-phosphates from 1.4 to 4.8 and from 0.5 to 1.4 for ATP and ADP, and GTP and GDP, respectively. No change in the adenosine and inosine le- 

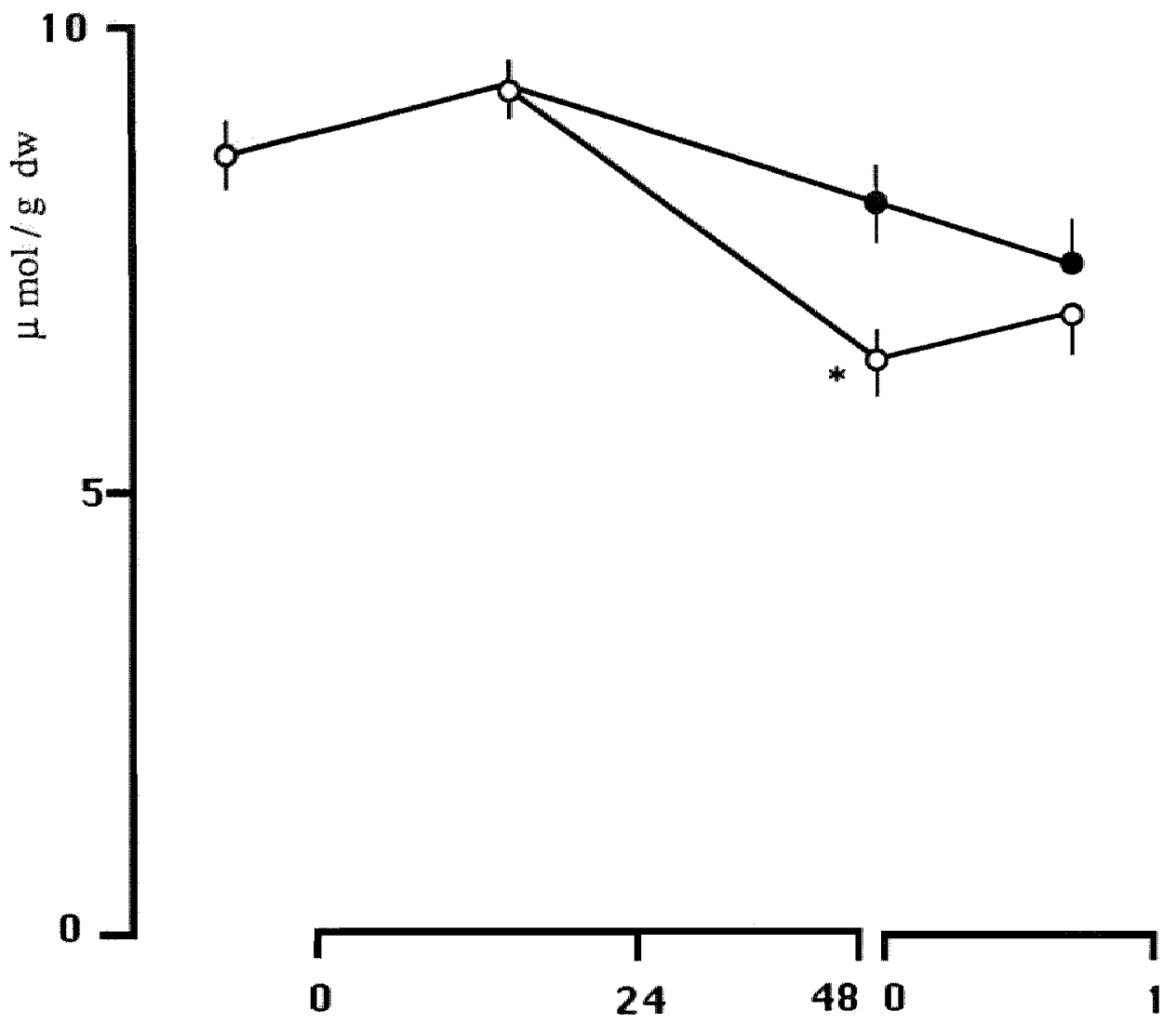

Cold storage

Reperfusion

( hours )

Figure 10.1.: Effect of intermediate normothermic perfusion on tissue nucleotide content (i.e. the sum of adenine nucleotides, guanine nucleotides, and IMP) during cold storage andl reperfusion in kidneys with 30 minutes of preceding warm ischemia. Symbols are open circles: kidneys with 30 minutes of preceding warm ischemia; closed cincles: kidneys with 30 minutes of preceding warm ischemia and intermediate normothermic perfusion. The asterisk indicates significance of the difference from corresponding values in kidneys with intermediate normothermic perfusion $(p<0.05)$. 
vels was observed. The small amount of hypoxanthine and xanthine disappeared during perfusion.

\section{Reimplantation}

The effect 60 minutes reperfusion following reimplantation after cold storage on kidney nucleotide levels is shown in table 10.2 and figure 10.1 and 10.2. After 60 minutes of reimplantation following 24 or 48 hours of cold storage the guanine nucleotide pool significantly increased. The adenine nucleotide pool remained unaffected in the 24 hours

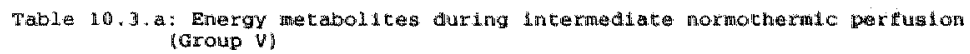

\begin{tabular}{|c|c|c|c|c|c|}
\hline ATP & ADP & ANAP & GTP & GIDP & Gas \\
\hline
\end{tabular}

\begin{tabular}{|c|c|c|c|c|c|c|}
\hline Betore & $0.6 \pm 0.0$ & $0.9 \pm 0.1$ & 5.0 .0 .3 & $0.2 \neq 0.0$ & $0.3 \pm 0.0$ & $0.8 \pm 0.0$ \\
\hline Atter & $2.5 \pm 0.2^{a}$ & $2.3 \pm 0.1^{18}$ & $2.4 \# 0.2^{a}$ & $0.1 * 0.01$ & $0.6 \pm 0 . a^{a}$ & $a .3 \pm 0.0^{\mathrm{a}}$ \\
\hline
\end{tabular}

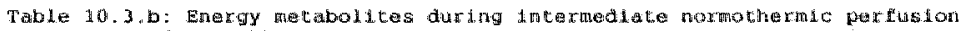
(Group V).

IMP $\quad$ ADO INO $\quad$ IN

$\begin{array}{lllllll}\text { sefore } & 2.2 \pm 0.2 & 0.1 \pm 0.0 & 0.1 \pm 0.0 & 1.0 \pm 0.1 & 1.0 \pm 0.2 \\ \text { After } & 0.6 \pm 0.32 & 0.2 \pm 0.1 & 0.1 \pm 0.0 & 0.1 \pm 0.040 .1 \pm 0.29\end{array}$

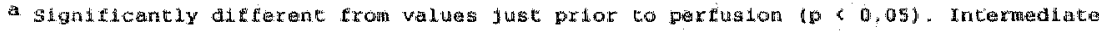
normothermic perfusion was performed after 30 minute of warm lichemite followed by 24 hours of cold storage. Walues are means $\mathrm{sem}, \mathrm{n} \approx 6$.

Blopstes were taken inmediately before and after 60 minutes of perfibion.
}

storage groups but dropped in the 48 hours storage groups. In both groups IMP levels were greatly reduced during reperfusion (table 10,2). When the cold storage period was preceded by 30 minutes of warm ischemia a, at some points, different pattern was observed. In contrast to the non-ischemic groups adenine nucleotide levels dropped in the 24 hours storage groups but remained unaffected in the 48 hours storage groups after 60 minutes of reperfusion. The effect on guanine nucleotide and IMP levels was not different from that observed in the non-ischemic groups. Reimplantation of kidneys stored for 48 hours with intermediate normothermic perfusion (group V) decreased the summated pool of adenine nucleotides, gwanine nucleotides, and IMP from $8.4 \pm 0.5$ to 7.2 $\pm 0.2 \mu \mathrm{mol} / \mathrm{g} \mathrm{dw}$ (figure 10.1). This decrease was mainly caused by a reduction in tissue IMP content (from $1.9 \pm 0.2$ to $0.4 \pm 0.1 \mu \mathrm{mol} / \mathrm{g} \mathrm{dW}$ at the end of storage ws postimplantation, respectively) since adenine nucleotide levels remained unchanged and guanine nucleotide levels showed a slight increase $(1.1 \pm 0.2$ vs $1.5 \pm 0.1 \mu \mathrm{mol} / \mathrm{g}$ $\mathrm{dw}$, respectively). It should be noted that the postimplantation values were not significantly different from these values in postimplantation biopsies obtained in the corre- 
sponding group without intermediate normothermic perfusion (figure 10.1). Interestingly, intermediate normothermic perfusion of the kidneys with preceding warm ischemia did not result in a loss of nucleotides (table 10.3) whereas reperfusion in situ after reimplantation was associated with a significant loss of $1.2 \mu \mathrm{mol} / \mathrm{g} \mathrm{dw}$ nucleotides within a comparable time interval, i.e. 60 minutes.

Special attention has been paid to the ability of the transplanted kidney to rephosphorylate the adenine monophosphate pool. In figure 10.2 the content of AMP and the sum of ATP and ADP during the various stages of the experimental procedures is shown. In group I and II the rephosphorylation expressed as the ratio of AMP and the sum of ATP

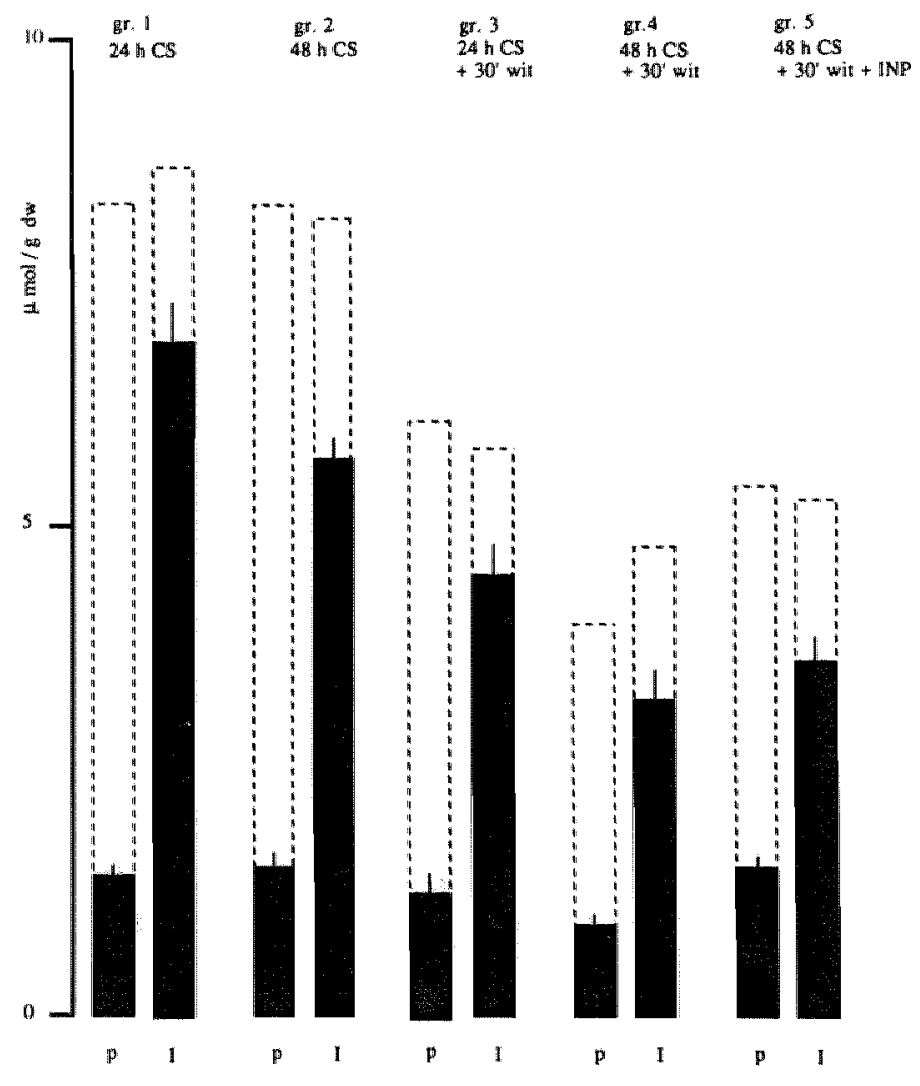

Figure 10.2.: Sum of ATP and ADP in renal tissue during cold storage and reperfusion following reimplantation (shaded bars). Biopsies were taken at the end of preservation (P) and 60 minutes after implantation (I). The dotted bars indicate the total adenine nucleotide pool including AMP (see also table 2). Values are means \pm SEM of 6 experiments in each group. The rephosphorylation expressed as the ratio of AMP vs the sum of ATP and ADP, was significantly higher in group I and II in comparison to the other groups $(\mathrm{p}<0.05)$. 
and ADP is significantly higher than in the other groups $(p<0.05)$. Notably, the ability to rephosphorylate is maintained in all cases.

\section{DISCUSSION}

The results of the present study are in agreement with previously lylylreported data indicating that the postimplantation viability of canine kidneys simply stored on ice is determined by both the length of storage time and preexisting warm ischemic injury (Johnson,1972;Pegg,1984). Storage time over 24 hours negatively influenced kidney viability. When the storage on ice was preceded by 30 minutes of warm ischemia viability was further depressed. It was, however, demonstrated in the present study that when kidneys damaged by preceding warm ischemia and subsequent storage on ice for 48 hours were subjected to an intermediate normothermic perfusion (i.e. after 24 hours of cold storage), posttransplantation viability was markedly improved. Recent experimental studies in rat and dog have shown that an intermediate normothermic perfusion halfway the cold storage period can increase the maximum length of storage time of fresh kidneys about twofold (Rijkmans,1984;Gaber,1987). It was unknown whether an intermediate normothermic perfusion is also beneficial to simple cold storage of ischemically injured kidneys. With respect to a possible relationship between tissue content of nucleotides and viability of the kidney some interesting phenomena were observed. The relationship, if any, between renal posttransplant viability and the tissue content of nucleotides as markers of the energy state of the organ has been subject of many debates (Gaudio,1986;Pegg,1978). The present findings indicate that the possible relationship is rather complex. First, nucleotide levels at the end of the cold storage period and just prior to transplantation of the kidney were not directly related in all cases to the ability of the kidney to regain function after reimplantation. Tissue guanine nucleotides and IMP were comparable in all groups studied at the end of the storage period despite marked differences in postimplantation viability. The content of adenine nucleotides provided more interesting information. At the end of the storage period high adenine nucleotide levels seemed to be not a condition sine qua non for posttransplant viability. Kidneys stored for 24 hours or 48 hours on ice had similar cortical adenine nucleotide levels but the percentage of organs showing posttransplant viability was 100 and $33 \%$, respectively. These findings suggest that the viability of the kidneys, as tested in the present unilateral transplant model, is lost prior to severe depletion of tissue adenine nucleotide levels. In the group with the lowest adenine nucleotide levels, i.e. 30 minutes of warm ischemia preceding 48 hours of cold storage, viability was completely absent. It is important to note that intermediate normothermic perfusion in this group improved significantly both end-storage adenine nucleotide levels and posttransplant viability. Although the latter observation suggests a relationship it is not proven that this relation is crucial. Second, the events occurring during the initial phase of reimplantation might also exert a critical effect on postimplant nucleotide levels and viability. For instance, the adenine nucleotide content significantly decreased in kidneys in which a moderate extent of previously inflicted damage can be expected, i.e. 48 hours storage without preceding warm ischemia (group II), 24 hours of storage with 30 minutes preceding warm ischemia (group III) and 48 hours of cold storage with 30 minutes warm ischemia and intermediate normothermic perfusion (group V). Although no definite explanation for the postimplant decrease of adenine nucleotides can be offered, we cannot exclude that 
during the initial reimplantation period additional damage will be inflicted on the kidney both influencing adenine nucleotide homeostasis and viability. One should keep in mind that reimplantation of the previously isolated kidney introduces a second period of warm ischemia due to the time required to perform the vascular anastomosis to complete the transplantation procedure. Lack of further decline in the least damaged kidneys (group I, 24 hours of storage) might be caused by a well preserved capability to withstand the period of ischemia during reimplantation. On the other hand, the lack of further decline in the most damaged kidneys ( 48 hours of storage with 30 minutes preceding ischemia) can be explained by the previously published observation that renal adenine nucleotide levels reach a nadir level of about 30\% of control (Maessen,1988). For unknown reasons adenine nucleotides in ischemic tissue will not readily decline below this experimentally established level. The efficacy of cold storage to preserve tissue nucleotide levels depends obviously on a variety of factors. From the present findings the conclusion can be drawn that cold storage on ice up to 48 hours is adequate to maintain the nucleotide content at a relatively high level. The difference between the values observed after 24 hours storage and control are most likely caused by the procedure of flushing prior to storage as has been demonstrated previously (Maessen,1987c). Warm ischemia preceding the cold storage period obviously attenuated the capability of the storage procedure to preserve tissue nucleotide levels. This notion is substantiated by the finding that in between 24 and 48 hours of cold storage tissue levels of nucleotides started to decline (table 10.2). Interestingly the negative effect of preexisting warm ischemia can be in part counteracted by intermediate normothermic perfusion for 2.5 hours half-way storage time. The mechanism underlying the apparent beneficial effect of intermediate normothermic perfusion cannot be clearly defined at this stage. A possible relationship between the better preservation of adenine nucleotide levels just prior to reimplantation and improved viability cannot be excluded. The present findings, however, are not entirely consistent with an original idea on the effect of intermediate normothermic perfusion suggesting that a restore of the energy metabolite content is the cause of the improved tissue viability (Rijkmans,1986). An increased salvage of the nucleotide content during storage following the intermediate normothermic perfusion was found. This increase however was small. Furthermore the increased salvage during the second 24 hours of cold storage did not prevent the decrease in the nucleotide pool during posttransplant reperfusion. The beneficial effect of intermediate normothermic perfusion may be related to a feature of energy metabolism which is not directly reflected by actual nucleotide levels or may be exerted by other factors than the energy status of the organ. In this respect, several factors may be considered. For instance, long-term withdrawal of the vascular endothelium from the interaction with blood constituents has been suggested to limit the length of preservation time (Pegg,1984;Southard,1985). Intermediate normothermic perfusion might in part meet this need of the endothelium and thus enhance the tolerance to hypothermia. During hypothermic storage a phase change of the lipid bilayers of the membrane occurs from a liquid crystalline to a gel state. This transition is dependent on temperature and the physical properties of individual phospholipids (Kruuv,1986). Membranes are normally protected by several mechanisms against heterogeneous distribution of phase changes within the membrane which will result in increased leakiness and disruption upon rewarming. The effectiveness of these adjustments, however, may faint during prolonged exposure to hypothermia (Hochach- 
$\mathrm{ka}, 1986)$. It is tempting to speculate that timely and temporarily rewarming of the membranes by intermediate normothermic perfusion may prevent the occurrence of phase separation and hence the loss of membrane fluidity. In conclusion, it is demonstrated in this study that it is possible to enhance the viability of ischemically injured kidneys prior to long term simple cold storage on ice. The importance of nucleotide homeostasis during hypothermic storage for posttransplant viability remains to be established.

\section{SUMMARY}

The effect of simple cold storage on ice with or without preceding warm ischemic injury on the energy metabolism and posttransplant viability of canine kidneys was examined in the present study. In addition, we investigated the possible beneficial effect of an intermediate normothermic perfusion half-way the storage period on the preservation of ischemically injured kidneys. Thirty mongrel dogs were allocated to 5 experimental groups. In group I and II kidneys were simply stored on ice for 24 and 48 hours, respectively. In group III and IV kidneys were additionally subjected to 30 minutes warm ischemia before storage. In group 5 kidneys were treated similar as in group IV, however, halfway the storage period an intermediate normothermic ex-vivo perfusion was performed. The effect of these procedures on renal viability was tested by autologous reimplantation of the kidneys. During implantation the contralateral kidney was immediately removed. In group I all animals survived whereas in group IV none of the animals survived. In group II, III, and V, 2 out of 6,1 out of 6 , and 3 out of 6 animals survived, respectively. The relationship, if any, between post-storage renal viability and the tissue levels of adenine nucleotides, guanine nucleotides, IMP and purine degradation products was assessed by measuring the content of these metabolites testin tissue specimen of the renal cortex, biopsied at various intervals during the experimental procedures. After an initial drop of about $30 \%$ in the content of adenine and guanine nucleotides and an increase in IMP these values remained constant during 48 hours of cold storage. In contrast to kidneys stored for 24 hours reimplantation of kidneys stored for 48 hours resulted in a significant decrease of adenine nucleotides following 60 minutes of in vivo reperfusion. 30 minutes of warm ischemia prior to cold storage lead to lower initial nucleotide levels at the start of the storage period. During the first 24 hours nucleotide levels did not change but a further decrease was observed during the following 24 hours of storage. Reimplantation after 24 hours of storage resulted in an additional decrease in the content of nucleotides. This post-storage decrease was absent after 48 hours of cold storage. Intermediate normothermic perfusion half-way the storage for 48 hours prevented significantly the drop in the nucleotide content as observed during the last 24 hours of storage in the corresponding control group. This nucleotide sparing effect did not increase the level of nucleotides at the end of 60 minutes of reperfusion following reimplantation. These findings suggest that the tissue nucleotide content at the end of cold storage is not necessarily predictive for viability upon implantation. In general, however, preexisting warm ischemic injury impairs renal viability following implantation and reduces the nucleotide sparing effect of cold storage. 
APPEARANCE OF ENZYMES IN PLASMA OR URINE FOLLOWING RENAL INJURY"

\section{INTRODUCTION}

A number of studies indicate that renal injury such as resulting from rejection after kidney transplantation, can be detected from urinary excretion of renal enzymes (Mattenheimer,1977;Plummer,1979). Earlier attempts to confirm renal injury from the enzyme activity in plasma were inconclusive (Ringoir,1968; Mattenheimer,1968; Raab,1972; Price, 1982), leading to the view that enzymes released from damaged kidney cells do not reach plasma. Recently methods have been developed for quantitative assessment of organ damage from accumulated enzyme activity in plasma. Calculation of the amount of enzyme in plasma, lost from damaged tissue, has been shown to permit differentiation between various forms of liver injury (Schmidt,1976) and also to permit the assessment of infarct size in patients with acute myocardial infarction (Simoons,1986).

A quantitative estimation of renal injury could be of importance in clinical transplantation. It may allow prediction of viability of donor kidneys and the evaluation of non-functioning kidneys after implantation. So far a quantitative relationship between enzyme activity in urine and the extent of renal injury has not been established. The amount of enzymes excreted in urine (Mattenheimer,1977;Plummer,1979) is usually negligible compared to the tissue content of these enzymes in the kidney. This might be explained by assuming that the actual renal cellular necrosis is too small to produce a considerable amount of enzyme loss. An alternative explanation might be that a major part of the enzyme released upon renal injury, does not reach the urinary system but is locally inactivated or enters the plasma. In this study these possibilities were verified.

An experimental model was developed to study the transport of enzyme released in the kidney following an artificial enzyme infusion into the cortical parenchyma of healthy and ischemically damaged, canine kidneys. The quantitative recovery of enzyme in plasma and urine was calculated. Subsequently the changes in plasma enzyme activity following an established large renal infarction were studied.

\section{MATERIALS AND METHODS}

Surgical procedure

Healthy mongrel dogs $(N=19)$ of either sex were used, weighing between 20 and $30 \mathrm{~kg}$ (Laboratory Animals Center, University of Limburg, The Netherlands). One day before the experiment animals were allowed free access only to water. Anaesthesia was induced with intravenous thiopentotal $20 \mathrm{mg}$ per $\mathrm{kg}$ body weight, and maintained with a nitrous oxide oxygen gas mixture. The effect of ventilation, performed by a positive pressure respirator, was monitored and adjusted according to bloodgas analysis (ABL, Radiometer Kopenhagen, Denmark) and ECG registration. The animals were placed on a thermostatic watermattress. A polyethylene catheter, inserted into the femoral vein, allowed blood sampling. After laparotomy through a midline abdominal incision the ureter of the left kidney was cannulated for urine collection. 


\section{Enzyme infusion}

A hollow needle with a fine cannula was tangentially inserted into the left kidney. The needle was removed leaving the cannula in the cortical zone. The cannula was connected to an external pressure transducer and records of pressure changes upon infusion were obtained on a multichannel recorder. A solution of purified porcine alanine amino transferase (E.C.2.6.1.2.) obtained from Sigma (St. Louis, MO) was infused into the parenchyma. A syringe pump delivered a constant infusion rate of $0.1 \mathrm{ml} / \mathrm{min}$. Evans blue was added to the infusion medium to allow a check on possible loss from the puncture site. It allowed location of the cannula tip at the end of the experiment. Blood and urine samples were collected at the start of the experiment, at the start of the infusion, and at 15 minutes intervals during and after the infusion. The last samples were taken sixty minutes after the end of the infusion. Finally the left kidney was removed and the correct position of the cannula was checked. The enzyme activity in plasma and urine samples was assayed spectrophotometrically at $25^{\circ} \mathrm{C}$ using a commercially available testkit (Boerhinger, Mannheim, FRG).

\section{Calculations}

The cumulative release of enzyme per litre of plasma $(Q(t))$ was calculated from plasma enzyme activities according to the equation:

$Q(t)=C(t)+E(t)+F C R$ of $C(t) d t(E .1)$. This equation is based on the two compartment model as shown in fig 11.1. The three terms of this equation represent the enzyme activity $\mathrm{C}(\mathrm{t})$ present in plasma at time $t$ (corrected for the normal steady state activity), the extravascular enzyme activity $E(t)$ at time $t$ and the fraction of enzyme activity which has been eliminated from plasma up to time t (FCR is the Fractional Catabolic Rate constant). The second term is calculated from the following equation;

$E(t)=T E R . \exp (-E R R . t)$ o $\int^{t} \exp (E R R t) C(t) d t(E .2)$. The Transcapillary Escape Rate constant (TER) and the Extravascular Return Rate constant (ERR) in the dog as well as the fractional catabolic rate constant of ALT were determined in previous studies (9) $\left(\mathrm{TER}=0.031 \mathrm{Hr}^{-1}\right.$, ERR $\left.=0.069 \mathrm{Hr}^{-1}, \mathrm{FCR}-\mathrm{ALT}=0.022 \mathrm{Hr}^{-1}\right)$. The validity of this model for the quantitative estimation of circulating enzymes has been shown elsewhere (Hermens,1982). The plasma volume of each animal was determined at the end of each experiment by intravenous injection of a bolus of the enzyme solution and by monitoring the subsequent changes in plasma activity during 15 minutes.

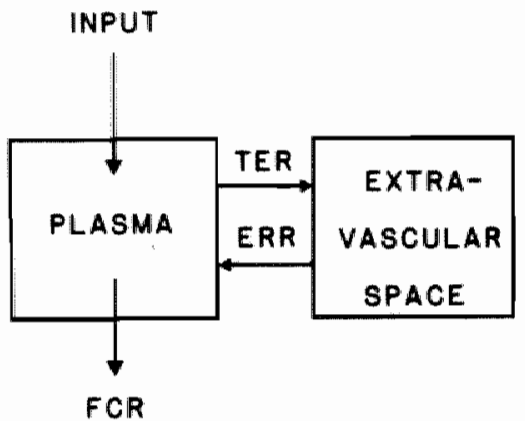

Figure 11.1.: Two-compartment model for circulating proteins. See text for explanation. 
Experimental groups

Group I $(n=7)$ : A sixty minute infusion was performed at an infusion rate of $0.1 \mathrm{ml} / \mathrm{min}$. The enzyme solution contained $94 \mathrm{U} / \mathrm{ml}$ (mean value) ALT. To study the possibility that the disappearance rate of the enzyme solution from the renal tissue was influenced by an increasing pressure in a growing pocket of enzyme suspension within the cortical tissue, a subgroup $I A(N=3)$ was introduced. In this subgroup the infusion was followed by a second sixty minute infusion at double infusion rate $(0.2 \mathrm{ml} / \mathrm{min})$ and with an enzyme solution at half the original concentration.

Group II $(n=3)$ : In this group the effect of ischaemia on the release of the infused enzymes was examined. Thirty minutes before the start of the infusion, one of the first two main branches of the renal artery was ligated. The infusion was performed in the ischemic area. Arterial supply and urine production were maintained in the remaining part of the kidney. At the end of the experiment the kidneys were dissected and angiograms were made after injection of Urografin (Schering AG, Berlin, Bergkamen, FRG) into the renal artery.

Group III $(n=5)$ : This group consisted of sham-treated animals, two of which received in addition to surgical manipulation an infusion without enzymes. This group was regarded as a control to a spontaneous and additional ALT release as a result of the surgical and infusion procedure. Group IV $(n=4)$ : In addition to the infusion studies the spontaneous plasma enzyme release of three cytosolic enzymes following renal infarction was monitored during seven days. The surgical procedure was performed as in Group II. Daily plasma samples were collected and enzyme activity was assayed spectrophotometrically at $25^{\circ} \mathrm{C}$ using commercially available testkits (Boehringer, Mannheim, FRG): lactate dehydrogenase (LDH, EC 1.1.1.27) aspartate aminotransferase (AST, EC 2.6.1.1.); glutamate dehydrogenase (GLDH, EC 1.4.1.2).

\section{RESULTS}

\section{Experimental study}

Insertion of a cannula to the renal cortex allowed the infusion of the enzyme suspension without leakage. Bleeding from the puncture site was minimal and stopped spontaneously within few minutes. In all animals the correct location of the cannula was confirmed by macroscopic sectioning of the kidney after the experiment. In the ischemic kidneys the effect of ligation of a renal artery branch and proper positioning of the cannula within the ischemic area was ascertained angiographically (Fig.11.2). During the infusion the pressure in the cannula ranged from 16 to $32 \mathrm{mmHg}$.The infusion of ALT in bealthy renal cortical tissue (Group I) resulted in rapid increase of plasma enzyme activity (Fig.11.3). Calculated recovery of ALT in plasma was $76 \pm 14 \%$ (mean \pm SEM) within 60 minutes after the end of the infusion. The cumulated enzyme activity in continuously collected urine samples did not exceed 1\% (data not given). A double infusion speed at half enzyme concentration in group LA did not alter the slope of the curve (Fig.11.3). The pressure in the cannula tip remained unchanged. These results demonstrate that the infused enzyme was transported predominantly to the plasma at a rapid rate, indepently of the infusion pressure. Not all enzyme was recovered during the observation time. The flattening of the curve suggests that further recovery of the enzyme was reduced or absent. Enzymes infused in a part of a kidney which is completely de- 


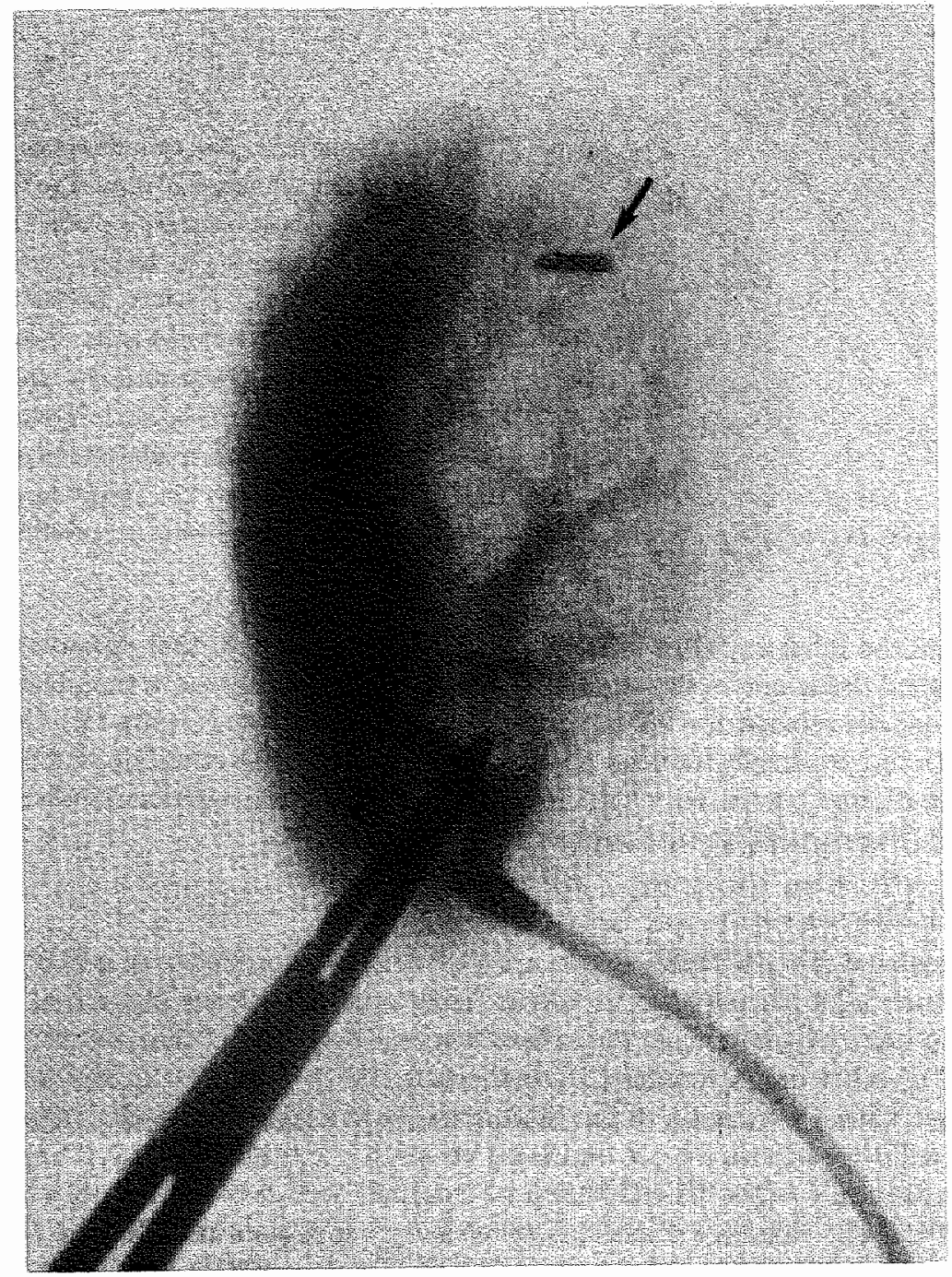

Figure 11.2: The effect of ligation of one single renal artery branch as shown by angiography in the ischemic model. The arrow indicates the position of the tip of the cannula.

void of arterial blood supply (Group II) also reached the plasma rapidly: The cumulative plasma release, two hours after start of the infusion, amounted to $89 \% \pm 7$ (mean \pm SEM) of the infused activity. The urinary accumulation was negligible (less than $1 \%$ in overall urine production). Apparently the absence of arterial blood supply did not influence the transport. The spontaneous release of renal enzymes in plasma following renal infarction is shown in Figure 11.4 (Group III). Renal infarction resulted in prolonged increase of activity of all three enzymes. Peak values of LDH and AST were obtained within $24 \mathrm{hrs}$ whereas GLDH concentrations reached their maximum between 


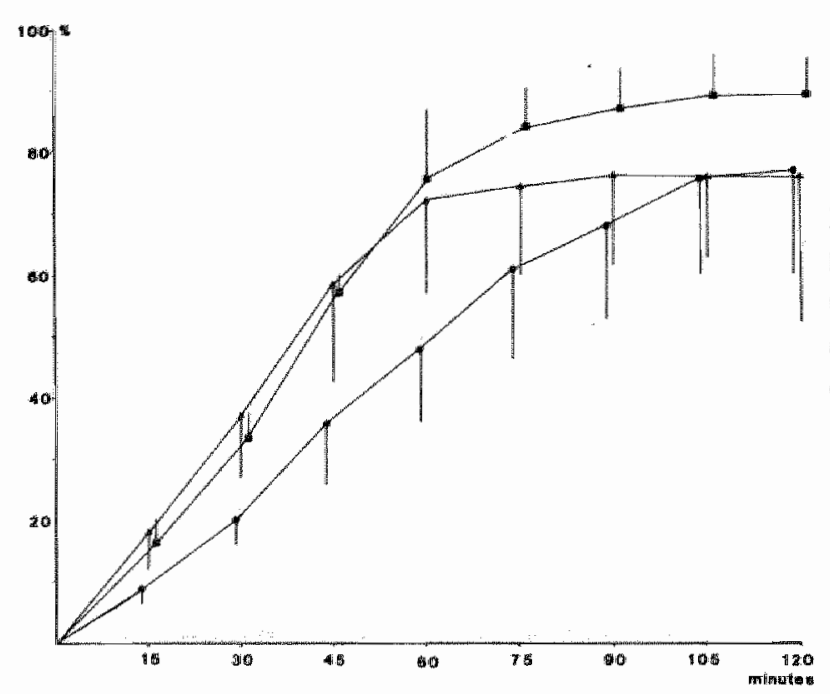

Figure 11.3: Plasma recovery of ALT following intrarenal infusion. The recovery is: the cumulative release in plasma, expressed as percemtage of the infused amount of enzyme. The infusions in healthy tissue (triangle; $N=4$ ) and ischemic tissue (rectangle; $N=3$ ) were performed from $t=0$ to $t=60$ minutes. In subgroup Ia (circle; $N=3$ ) the infusion was continued at doubled rate from $t=60$ to $t=120$. Mean values are given with either positive or negative SEM values.

the first and the third day after infarction. These results suggest that enzyme released from damaged tissue enters the interstitial space and hence appear in the plasma.

The spontaneous release of ALT during the surgical procedure (Group IV) did not interfere with the artificially induced changes of ALT activity as could be demonstrated in the sham-treated control animals (Group III). Plasma levels remained undisturbed at $24 \pm 3 \mathrm{U} / \mathrm{h}$ (mean $\pm \mathrm{SEM}$ ) (data not shown).

\section{DISCUSSION}

In the assessment of renal damage there are sound reasons for the use of urinary enzyme excretion instead of plasma concentrations. Experimental evidence shows that enzymes released in urine originate from the kidney whereas elevation of plasma enzymes may be caused by other organ systems (Mattenheimer,1977). More over quantitatively collected urine allows estimation of the total quantity of enzyme released, provided that certain conditions of urinary $\mathrm{pH}$ and ionic strength are fulfilled (Jung,1983). Plasma enzyme values only allow the estimation of cumulative release if a proper circulatory model is used and values of transcapillary exchange constants and the rate of enzyme elimination from plasma, have been determined (Hermens, 1982).

Furthermore, it should be realized that a slight elevation of plasma enzyme levels may still reflect the release of a large amount of enzyme if this elevation lasts for several days because a large amount of enzyme may have been eliminated from plasma in such a period. It may remain unnoticed, e.g. when a patient has a serum activity of aspartate aminotransferase (AST) of $30 \mathrm{U} / \mathrm{L}$ during the first two days after kidney transplantation because such a value is within normal range. However, if the preoperative serum activity in such a patient was $10 \mathrm{U} / \mathrm{L}$, an elevation to $30 \mathrm{U} / 1$ comprises a total release in plasma of about $300 \mathrm{U}$ of AST, corresponding approximately to 10 grams of renal cortex. The present study demonstrates that an artificially induced increase of enzyme content in the renal interstitium results almost immediately in release of these enzymes in plasma and not in urine. The infusion into permanent ischemic tissue caused a similar enzyme recovery in plasma as the infusion in healthy tissue. In this respect it must be noted that 


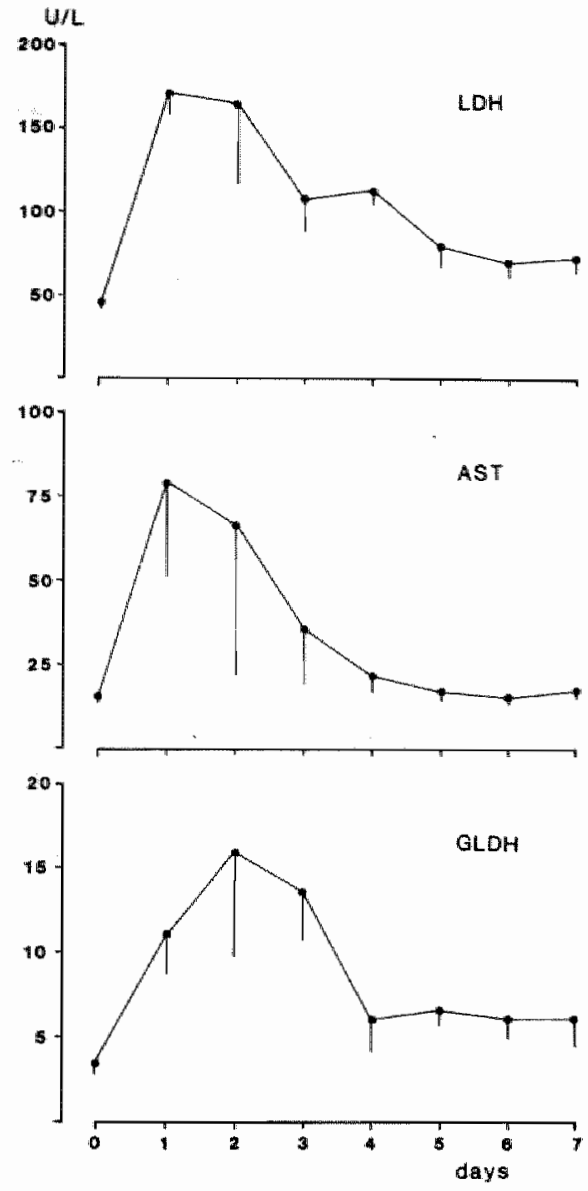

Figure 11.4.: LDH, AST and GLDH activity in plasma during partial renal infarction. Mean values of four animals are given with negative SEM vallues.

the intrarenal venous system consists of a freely anastomotic drainage network throughout the kidney which is obviously not impaired in this ischemic model in contrast to the arterial system which is totally blocked in the area of supply distal to the ligated branch of the renal artery (Fig.11.2) (Beeuwkes,1980). Although there was only a short delay between the rate of accumulation in plasma and the infusion rate, not all the infused enzyme was recovered. Some of the enzyme may have accumulated in the interstitium or the lymphatic system (Szabo,1976). The rapid release of the enzyme might have been a pressure effect. Such a pressure-induced transport could be quite different from the normal physiological transport to plasma of interstitially released enzymes. Doubling of the infusion rate and simultaneous lowering of the enzyme concentration to half the original concentration did not increase the enzyme recovery rate. A pressure artefact would have been apparent from an increase in the slope of the appearance curve. However, the pressure in the cannula tip did not increase during the second infusion. Apparently the pressure did not surpass the compliance of the renal tissue.

Subsequently it was shown that during actual renal infarction, spontaneously released 
enzymes indeed appeared in plasma. Theoretically tubular cells, that are mostly considered affected during ischaemia, can loose their enzymes at the luminal side or at the basal or basolateral side, but during ischaemia tubules have been demonstrated to be blocked and the integrity of the basement membrane to be disrupted (Solez,1984), favouring enzyme release into the peritubular interstitium and subsequently in plasma.

In contrast to the rapid transport of enzyme to plasma demonstrated by the infusions, the enzyme release following renal infarction seemed to proceed for a considerable period of time (Fig.11.4). Apparently the prolonged elevation of plasma enzyme levels reflects ongoing necrosis instead of a rapid wash-out phenomenon.

In our clinical research program we are attempting to determine the quantitative release of enzyme from transplanted kidneys to allow estimation of pre-existing damage as well as damage following rejection or immunosuppressive therapy. As indicated in other studies (Price,1987) attention was focussed on the determination of enzyme activity in urine. Our above mentioned results however brought about some doubts about the quantitative relevance of enzyme excreted in the urine.

In conclusion our results show that in dogs enzymes are quantitatively recovered from the renal interstitium in plasma rather than urine and that renal cell death in dogs results in prompt appearance of intracellular renal enzymes in plasma.

\section{SUMMARY}

The present study evaluates the relevance of plasma enzyme activity in the quantitative assessment of renal injury. In a canine model enzyme release from kidneys to plasma and urine was studied following an artificial parenchymal enzyme infusion in healthy and ischemic kidneys. The cumulative enzyme activity in plasma and urine was calculated. Within one hour after the start of the infusion, $80 \%$ of the infused enzyme was recovered in plasma whereas urinary accumulation was negligible. A similar enzyme distribution between these compartments was obtained if the kidneys were ischemically damaged before the start of the infusion. Thus enzymes released into the renal cortical interstitium are very rapidly transported to the vascular system and are quantitatively recovered in plasma. Experimental renal infarction revealed that cell death due to ischaemia also resulted in enzyme accumulation in plasma. 


\section{CHAPTER 12}

\section{ESTIMATION OF RENAL ISCHEMIC DAMAGE IN THE CONSCIOUS DOG FROM QUANTITATED ENZYME LEAKAGE IN PLASMA ${ }^{1}$}

\section{INTRODUCTION}

The role of structural, metabolic, and functional disturbances in ischemic renal tissue on the development of transient or persistent renal failure is incompletely understood (Solez,1984). Consequently, single parameters to evaluate the extent of renal ischemic injury are difficult to establish (Fahy,1982).

Loss of integrity of cellular membranes is considered to be a key feature of ischemic injury (Hochachka,1986). Many pathological signs that have been observed can be attributed to alterations in the homeostasis between intra- and extra cellular compartments. Disruption of the cellular membranes may lead to leakage of macromolecules including enzymes. The loss of enzymes from ischemic tissue has been used to estimate the extent of ischemic injury in studies on myocardial infarction by calculation of the cumulative amount of enzymes appearing in plasma (Willems, 1985; Shell,1971). This procedure is based on the assumption that enzyme activity lost from the myocardium is recovered in plasma. During renal infarction increased enzyme activity has been found in urine and used as an indicator of tubular damage (Price,1982). Data from a recent study, however, suggested that the bulk of renal enzyme release after infarction enters into the plasma and that only a minor part of the enzyme activity appears in the urinary system (Maessen,1987). This study was therefore designed to obtain a quantitative comparison of the enzyme activity lost from renal tissue and the leakage of enzymes from the kidney into the circulation. The cumulative amount of enzyme entering the plasma was calculated, using a two-compartment model, and compared to the total enzyme activity depleted from the kidney (Hermens,1982). It is demonstrated that ischemia-induced loss of enzymes from renal tissue can be estimated from serially determined plasma activities for cytosolic AST.

\section{MATERIALS AND METHODS}

\section{Animals}

Experiments were performed on inbred beagles of both sexes weighing between 8 and $21 \mathrm{~kg}$ (Laboratory Animals Center, University of Limburg, The Netherlands). Anesthesia was induced with thiopental ( $20 \mathrm{mg}$ per $\mathrm{kg}$ body weight) and maintained with a nitrous oxide-oxygen gas mixture, delivered continuously by a positive pressure respirator via an endotracheal tube. Body temperature was kept constant with a thermostatic watermattress. One day before surgery the animals were fasted, given only free access to water. In each animal a polyethylene catheter was inserted in the left jugular vein and channelled subcutaneously to the neck to allow blood sampling.

Experimentall protocol

The effect of ischemia on renal tissue enzyme activity and the rellease of enzymes in the 
circulation was studied in two different ways. In a first series of experiments a left subcostal incision was performed through which the left renal artery was carefully dissected from surrounding tissues. An inflatable balloon occluder was mounted on the renal artery. The balloon catheter was exteriorized in the neck. The right kidney was removed through a right subcostal incision. The animals were allowed to recover for 5 days. At day 6 the balloon was inflated in the non-sedated and freely moving animal. After 90 minutes of renal artery occlusion the blood supply to the kidney was re-established.hedhed The animals were sacrificed following 48 hours reperfusion. The kidneys were removed and stored at $-80^{\circ} \mathrm{C}$ for further analysis.

In a second series of experiments the same protocol was performed with two changes. An occluder device was implanted that allowed permanent vessel occlusion. The renal artery was not totally occluded but the device was mounted on the left main branch of the renal artery to induce only partial renal ischemia. The occlusion was checked before the animals were sacrificed by the infusion of a large amount of Evans blue into the aorta. The kidneys were removed and cut into slices. The stained tissue part was macroscopically dissected from the non-coloured part. In these experiments the right kidney was removed simultaneously to the implantation of the occluder. Individual control values for renal enzyme activities were determined in the contralateral kidneys in both groups.

\section{Enzyme activity in renal tissue and in plasma}

Blood samples were obtained one hour before and $0,1,2,4,6,8,12,16,20,24,30,36$, 42 , and 48 hours following the onset of ischemia. Kidneys were dissected in samples of 5-6 $\mathrm{g}$ wet weight of tissue. These samples were completely homogenized at $4^{0} \mathrm{C}$ with an ultra-turrax in $15 \mathrm{ml}$ of Tris buffer $0.05 \mathrm{M}, \mathrm{pH} 8.5$ containing $0.05 \mathrm{M} \mathrm{NaCl}$ and $0.015 \mathrm{mM}$ pyridoxal phosphate, and subsequently ultrasonificated. The cortical and medullar region were handled separately. Homogenates were stored at $-20^{\circ} \mathrm{C}$ until analysis of enzyme activity. It was verified that the degree of enzyme extraction from tissue did not depend on sample size. Errors introduced by changes in tissue water content were checked by calculation of tissue dry weight. Control values were determined in freshly excised kidneys obtained from healthy dogs $(\mathrm{N}=21)$. Activities of aspartate aminotransferase (AST,EC 2.6.1.1) and lactate dehydrogenase (LDH,EC 1.1.1.27) were assayed spectrophotometrically at $25^{\circ} \mathrm{C}$ using commercially available test kits (Boehringer, Mannheim, FRG) and a Cobas Bio automatic analyzer (Hoffmann-Laroche, Basle, Switserland). The ratio of cytosolic to mitochondrial AST was determined with an immunoassay (Eiken Chemical Co., LTD, Tokyo, Japan). No release of mitochondrial AST into plasma was found after ischemic renal injury. Total AST activities in plasma are therefore shown.

\section{Quantitation of plasma enzyme activity}

The cumulative release of enzyme actitvity in plasma during a period of time was calculated according to a two-compartment model for circulating proteins. This model has been described previously (Hermens,1982;Maessen,1987b). Briefly: The cumulative amount of enzyme released up to time $t$ per litre of plasma, $Q(t)$, is represented by $\mathrm{Q}(\mathrm{t})=\mathrm{C}(\mathrm{t})+\mathrm{E}(\mathrm{t})+\mathrm{FCR} .0 \int^{\mathrm{t}} \mathrm{C}(\mathrm{t}) \mathrm{dt}$

in which $\mathrm{C}(\mathrm{t})$ presents the enzyme activity in plasma (corrected for the normal steadystate activity) and $\mathrm{E}(\mathrm{t})$ the extravascular enzyme activity, both at time $t$. The last term 
gives the enzyme activity which has been eliminated from plasma up to time t (FCR is the fractional catabolic rate constant). The extravascular enzyme activity is calculated as:

$E(t)=T E R \cdot \exp (-E R R . t) \circ \int^{t} \exp (E R R . t) C(t) d t$

in which TER (transcapillary escape rate constant) and ERR (extravascular return rate constant) have been determined in dog in previous studies (Hermens, 1982). Values of TER $=0.032 \mathrm{~h}^{-1}$ and ERR $=0.068 \mathrm{~h}^{-1}$ were used. To perform these calculations the fractional catabolic rates have to be determined. Total release was calculated by multiplication of $Q(t)$ with the plasma volume of the individual animal calculated from body weight $(47 \mathrm{ml} / \mathrm{kg})$.

Fractional catabolic rate

The intravascular elimination of LDH in dog was determined by monitoring the disappearance of enzyme activity in plasma following a bolus injection of enzyme preparations. These determinations were performed in normal healthy dogs as well as in dogs with renal ischemic injury. Enzymes were purified from kidneys that were harvested from other dogs. To this purpose kidneys were homogenized and centrifuged for 30 minutes at $1200 \mathrm{~g}$ and $4^{\circ} \mathrm{C}$. The supernatant was again centrifuged for 2 hours at $100,000 \mathrm{~g}$ and $4^{\circ} \mathrm{C}$. Enzyme activities in plasma were measured at regular intervals up to 24 hours after bolus injection.

Data analysis

The significance of differences of mean values were estimated with the Student's T-test

\section{RESULTS}

Tissue enzyme depletion

The effect of ischemia on renal tissue content of LDH and AST was studied 48 hours following the onset of the ischemic insult (see table 12.1). Transient vascular occlusion during 90 minutes caused an average decrease of cortical LDH levells from $234 \pm 46$ to $117 \pm 11.8 \mathrm{U} / \mathrm{g}$ while AST levels decreased from to $20 \pm 3.0 \mathrm{U} / \mathrm{g}$ (mean $\pm \mathrm{SEM}, \mathbf{n}=$ 9). In the medulla, the LDH and AST content were reduced from $125 \pm 5.6$ to $86 \pm 4.6$ and from $24 \pm 1.4$ to $9.6 \pm 1.2$, respectively.

Following 48 hours permanent occlusion of the blood supply to one half of the kidney the tissue enzyme content in this part was $140 \pm 11.7$ and $19 \pm 1.3 \mathrm{U} / \mathrm{g}$ for cortical LDH and AST, respectively $(n=5)$. In the medulla, ischemic LDH and AST levels were 85 \pm 3.6 and $15 \pm 1.1$, respectively. The mAST/cAST ratio was 0.66 in ischemic as well as in non-ischemic tissues.

\section{Edema}

Tissue water content was not markedly influenced by ischemic injury after 48 hours. In kidneys with transient ischemia the percentage dry weight of the cortex was similar to contralateral control kidneys (19.9 \pm 0.1 vs. $20.0 \pm 0.3$, mean $\pm S E M)$ and slightly increased in the medulla $(17.4 \pm 0.7$ vs. $15.5 \pm 0.2)$. In permanently ischemically damaged tissue the percentage dry weight decreased from $20.0 \pm 0.3$ (in control kidneys) to $18.4 \pm 0.2$ (significant at $p<0.05$ ) in the cortex and it did not change in the medulla (15.1 \pm 0.2 vs. $15.5 æ 0.2$ ). In individual experiments differences in dry weight between experimental and control kidneys always remained less than $10 \%$. Corrections for the oc- 


\begin{tabular}{|c|c|c|c|c|c|c|c|c|}
\hline \multirow[t]{2}{*}{ Exper laterent } & \multirow[t]{2}{*}{ 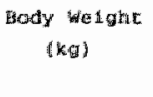 } & \multirow[t]{2}{*}{$\begin{array}{c}\text { Elagma } \\
\text { Worutio (L) }\end{array}$} & \multicolumn{2}{|c|}{$\begin{array}{l}\text { Renal tiggue } \\
\qquad(g):\end{array}$} & \multicolumn{2}{|c|}{$\begin{array}{l}\text { Control enzymat } \\
\text { cortex }\end{array}$} & \multicolumn{2}{|c|}{ 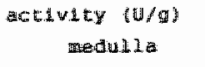 } \\
\hline & & & $\cos \theta \cos$ & meduld a & LDH & CAST & LDH & $\operatorname{chs} 1$ \\
\hline \multicolumn{9}{|c|}{ 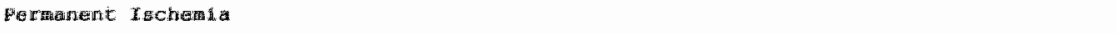 } \\
\hline 1 & 8 & 0.38 & 14.8 & 2.2 & $24+4$ & 13 & 目 28 & 6 \\
\hline 2 & 12 & 0.56 & 15.5 & 2.6 & 284 & 13 & 131 & 8 \\
\hline 3 & 12 & 0.56 & 18.9 & 5.2 & 267 & 14 & 123 & 7 \\
\hline 4 & 10 & 0.47 & 19.6 & 7.4 & 276 & 14 & 127 & 8 \\
\hline \multicolumn{9}{|c|}{ 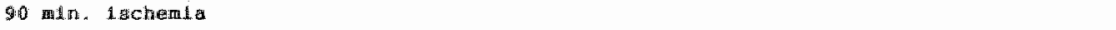 } \\
\hline 5 & 1. & 0.61 & 12.6 & 1.0 & 260 & 13 & I $\$ 0$ & 8 \\
\hline E & 21 & 0.99 & 70.3 & 20.7 & 180 & 14 & 79 & 5 \\
\hline$\gamma$ & 15 & 0.71 & 30.8 & 7.1 & 202 & 18 & 133 & 9 \\
\hline 8 & 1.8 & 0.815 & 22.1 & 7.4 & 178 & 18 & 146 & 11 \\
\hline 9 & 15 & 0.71 & 30.4 & 4.2 & 212 & 11 & 124 & $\$$ \\
\hline 10 & 13 & 0.61 & 24.7 & 2.0 & 170 & 13 & a) & 7 \\
\hline 11 & 16 & 0.75 & 37.5 & 6.4 & 221 & 18 & 254 & 10 \\
\hline 12 & 15 & $0.7 \mathrm{~d}$ & 32.2 & 4.3 & 262 & 18 & 151 & 10 \\
\hline 13 & 16 & 0.75 & 40.2 & 6.0 & 203 & 1.9 & 127 & 30 \\
\hline 1.4 & 14 & 0.616 & 27.5 & $4 \times 2$ & 321 & 17 & 119 & 30 \\
\hline Mentar 世 & $\Leftrightarrow$ & & & & $234 \pm 46$ & $15 \pm 0.5$ & $125 \pm 21$ & 6. $1 \neq 0.5$ \\
\hline
\end{tabular}

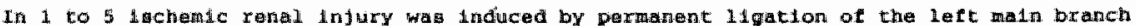
of the renal arterer.

The welght of renal tague that was chus rendered 1schemle $1 \mathrm{~g}$ ghown here. In 6 to 14 igchemic renal indury was induced by clamping of the renal vessels durimg 90 minutes

currence of edema were therefore omitted.

Changes in plasma enzyme activity

The changes in time of the plasma enzyme activity following renal infarction are shown in figure 12.1. Within one hour of reperfusion following 90 minutes of ischemia a rapid increase of plasma LDH and AST activity was observed. Between 4 to 8 hours the increase of activity reached peak levels and then gradually returned to pre-ischemic levels within 48 hours. In animals with permanent renal ischemia the rate of increase of enzyme activity was markedly slower and the period of release much longer. Thirty hours after the onset of ischemia a second continuous rise of plasma LDH activity was found up to 48 hours. The AST activity did not parallel this increase, suggesting that the additional rellease of $L D H$ is not from the kidney.

Fractional catabolic rate

The fractional catabolic rate of $\mathrm{LDH}$ activity in the circulation was calculated from the disappearance curves in plasma following the injection of a known amount of enzyme activity in healthy dogs. Thus a FCR of for LDH of $0.78 \pm 0.07 \mathrm{~h}^{-1}$ was obtained (mean $\pm \mathrm{SEM}, \mathbf{n}=9$ ). This value did not change if determined in dogs with ischemically damaged kidneys. In previous studies a FCR of $0.21 \mathrm{~h}^{-1}$ has been calculated for AST in dog (Hermens,1982). This value was used in the present experiments.

Cumulative plasma enzyme activity 


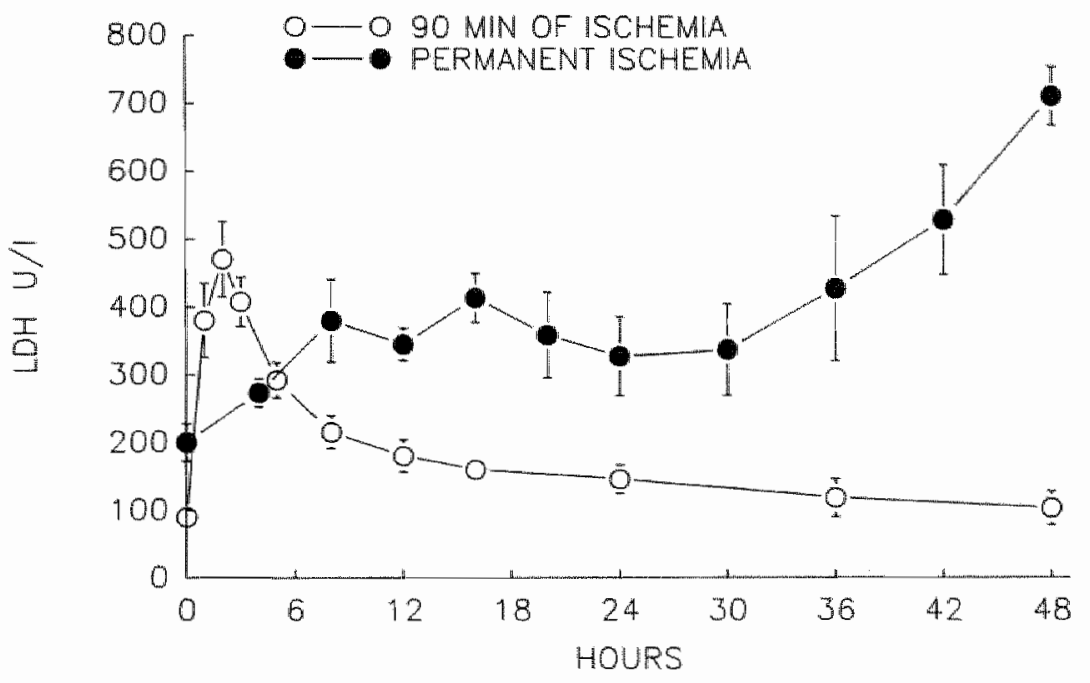

Figure 12.1.: Time course of $L D H$ and AST plasma activities following renal ischemia. Mean values $\pm S E M$ are given as in the next figures.

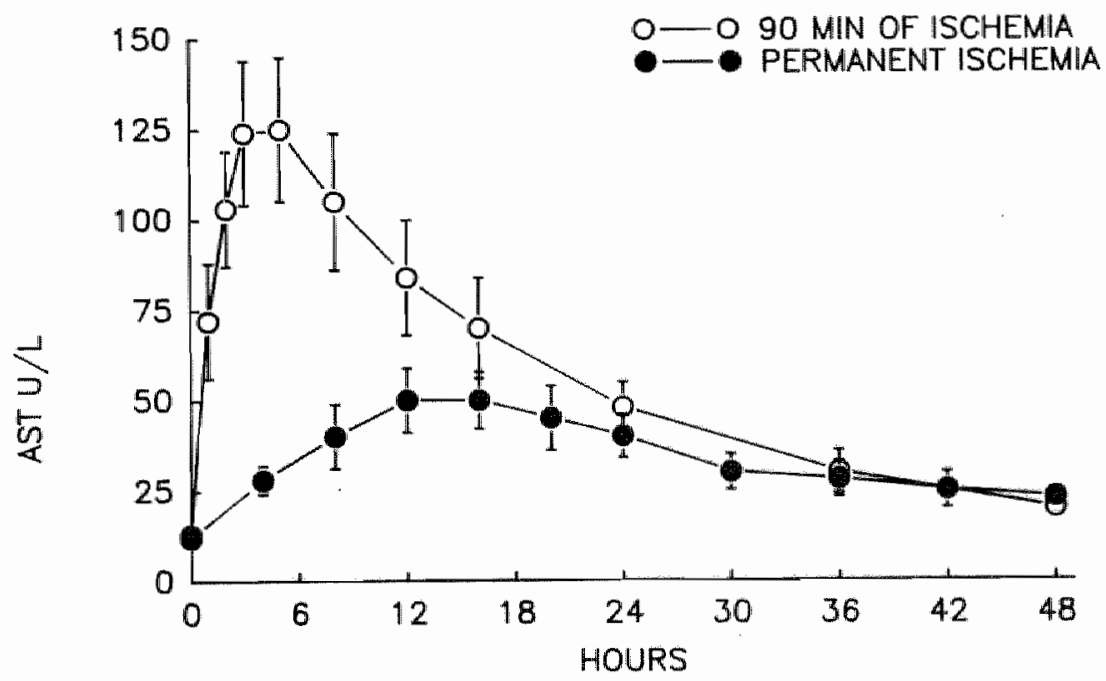

Using these values the total amount of enzyme activity that was recovered in plasma following renal ischemia was callculated (figure 12.2 and table 12.1). The cumulative plasma enzyme activity was expressed in gram equivalents of renal tissue based on the ratio of the quantitative amount of enzyme activity in plasma after some time and the enzyme activity in one gram of renal tissue. In dogs following 90 minutes of ischemia the cumulative plasma LDH activity expressed in gram equivalents remained below correspond- 


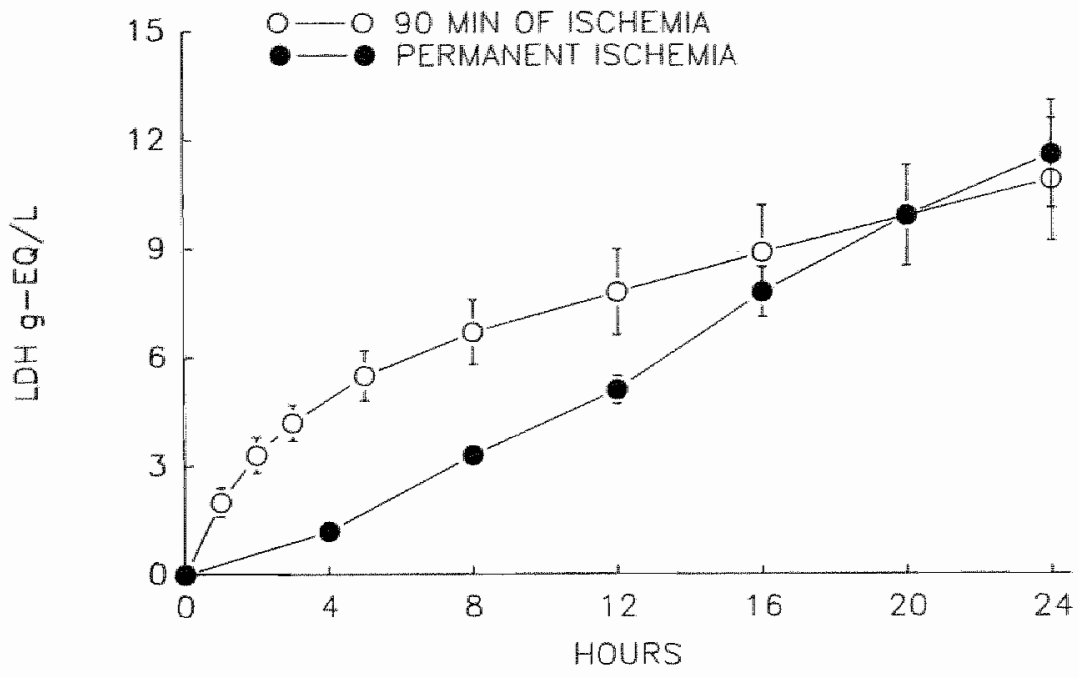

Figure 12.2: Cumulative release of LDH and AST following renal ischemia, expressed in gram-equivalents of renal tissue. It should be noted that in experiments with permanent ischemia only half of the total kidney was rendered ischemic (See materials and methods section),

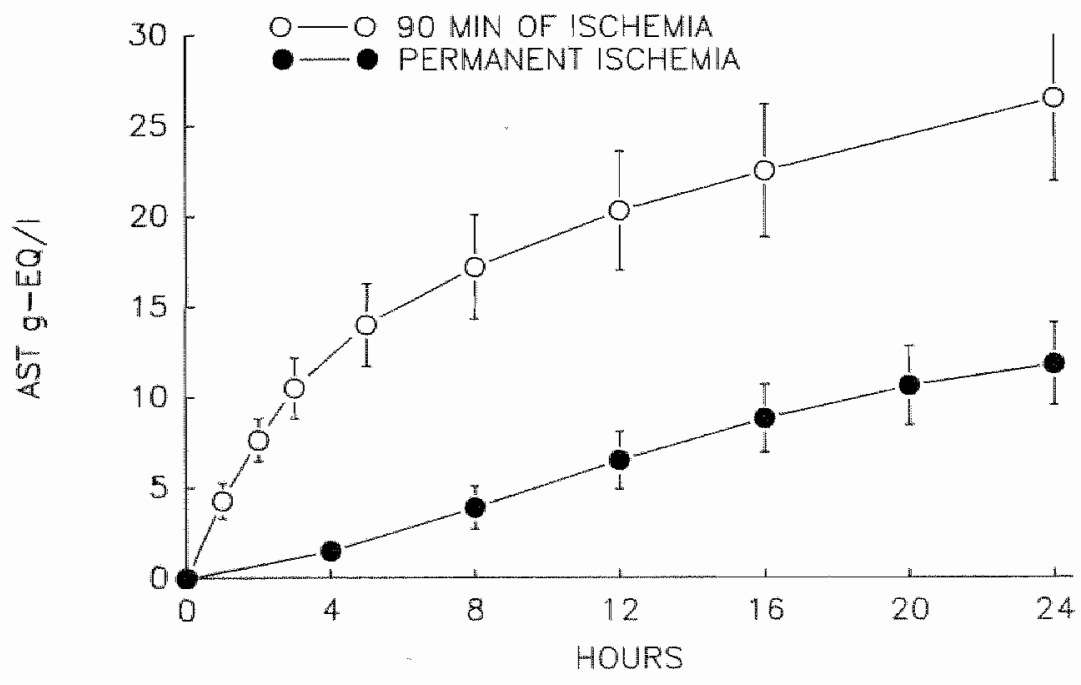

ing AST values from the onset of reperfusion onwards. In animals with permanent renal ischemia the appearance rate of enzyme in plasma was much more gradual and lacked the sudden burst of release after reperfusion. Within 2 hours no significant increase of plasma enzyme activity was observed. In contrast to the transient ischemically damaged kidneys, the cumulative plasma activity of $\mathrm{LDH}$ and AST was similar, in a quantitative 


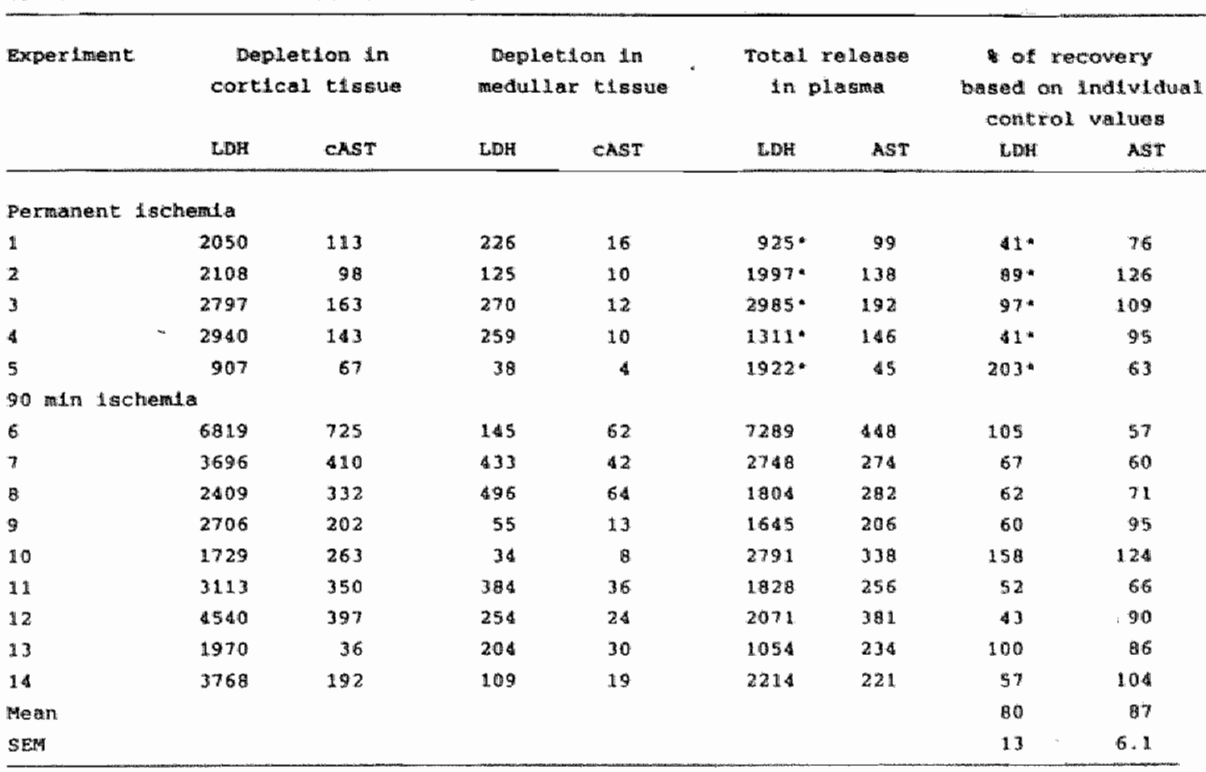

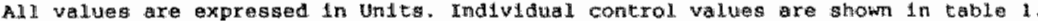

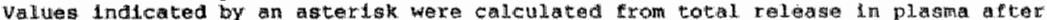
30 houx (see text).

way and with respect to the time course of the recovery.

Tissue enzyme loss and plasma enzyme recovery

The total amount of enzyme activity that was lost from the transient ischemically damaged kidneys as well as from the permanently ischemic kidneys were calculated after 48 hours and compared to the total amount of enzyme that was recovered in plasma during this time interval (table 12.1). It was thus found that on average $80 \%$ of the $\mathrm{LDH} \mathrm{ac}$ tivity that was lost from the ischemic tissue could be found back in the plasma. The AST recovery in plasma was about $90 \%$. These values for AST recovery were found both in kidneys with transient and permanent ischemic damage. This recovery was already largely completed after 24 hours. In table 12.2 it is shown that the recovery in plasma was markedly less complete in kidneys with extensive infarcted areas of more than two third of the total renal tissue as appeared from the strong tissue enzyme depletion. These differences are expressed in figure 12.3 in which the loss of tissue enzyme activity in individual experiments, expressed in gram equivalents of renal tissue is plotted against the corresponding cumulative plasma AST activity also expressed in gram equivalents of renal tissue.

\section{Methodological errors}

The calculations of enzyme recovery are subject to large methodological errors. The normal pre-ischemic enzyme activity in the kidney is known with an uncertainty of at least 
$10 \%$. As the total depletion of tissue enzyme activity is about $50 \%$, this uncertainty implies a 20\% error in calculated renal enzyme loss. A similar error is present in calculated cumulative release of enzymes in plasma. This error is mainly introduced by the biological variation in FCR and again has a magnitude of the order of $20 \%$. Assuming that these two errors are independent, the overall error in calculated recovery will be of the order of $\sqrt{ } 20^{2}+20^{2}=28 \%$ (see table 12.1).

\section{DISCUSSION}

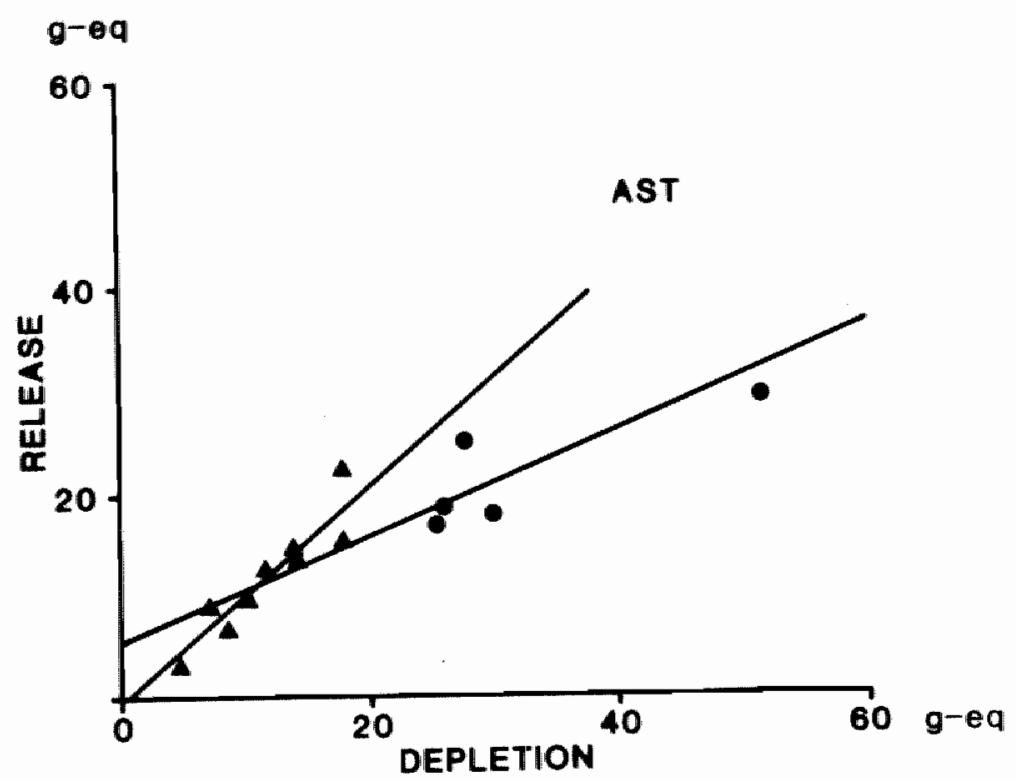

Figure 12.3.: Correlation between the cumulative release of AST and the depletion of tissue AST activity both expressed in gram equivalents of renal tissue. The correlation coefficient is 0.88 (circles and triangles) and 0.93 (triangles) if only kidneys with an infarct size less than two third of the total kidney are considered.

The diagnostic role of changes in plasma enzyme activity with respect to renal disease is generally considered limited and inferior to the importance of changes in enzyme activity in urine (Mattenheimer,1977). Recently it was demonstrated that in case of renal ischemic injury the opposite may be true (Maessen,1977). There are at least two sound reasons to reconsider the usefulness of plasma enzyme activity determinations. First, following renal infarction a number of intracellular enzymes appear in the blood circulation and only to a small extent in the urinary system. Second, in studies on myocardial infarct size a valid method has been developed to quantitate the amount of enzyme that is released from an organ into the circulation during a period of time (Willems,1985). The present study shows that with this method the amount of enzyme that appears in the circulation following renal ischemia can be quantitated in a chronically instrumented dog model. 
The effect of 90 minutes transient ischemia on tissue enzyme activity was more or less equal to the effect of permanent ischemia to one half of a kidney. When evaluated after 48 hours the tissue had lost approximately $50 \%$ of their LDH and AST activity. In the medulla the depletion of enzyme activity was significantly less than in the cortex. Only the medullar AST activity in kidneys after transient ischemic injury was similarly reduced. Others, using a transient ischemic kidney model have found that part of the medullar region is particular prone to ischemic injury (Mason, 1987).

The question is raised whether the remaining enzyme activity that is measured when depletion has been completed represents the normal enzyme activity of remaining non-injured cells and is thus predictive for the viability of the kidney. This question is extensively disputed in heart and liver studies (Reimer,1984;Hermens,1982). Enzymatic infarct size quantitation is usually interpreted by assuming that in ischemic tissue irreversible damaged cells loose their complete cytosolic enzyme content whereas the remaining cells maintain their normal enzyme content. In studies on liver damage however cells are considered in part to be transiently leaky as a result of a damaging impetus and to loose enzyme without being actually irreversibly damaged. In the present study no direct proof can be provided as to whether the latter mechanism, is also found in ischemically damaged renal tissue. In a previous study however, we demonstrated in a similar experimental model that kidneys subjected to 90 minutes of ischemia are able to regain normal function if they are allowed to recover for at least four weeks (Maessen,19881). So despite a loss of cytosolic enzyme activity of about $50 \%$ the organ as a whole can not be considered non-viable. In contrast, the enzyme activity in the renal tissue that is permanent ischemically damaged further depletes in time and eventually turns into scar tissue in about four weeks (Unpublished observation). Since the enzyme markers used in this study are by no means kidney specific it is conceivable that as a result of experimental procedures changes in plasma enzyme activity are troubled by simultaneous enzyme release from non-renal sources. In previous experiments, plasma enzyme activity changes were observed in animals with sham renal ischemia, of LDH, AST, and CK with a similar time course as in experiments including renal ischemia. Liver and heart could be ruled out as a major source of error since no increase of GGT and ALT was found. Because of a high plasma CK activity, damage to the skeletal muscle system offered the most likely cause of additional enzyme release. It appeared possible to discriminate the skeletal muscle enzyme release from possible renall enzyme release using the correlation between CK release and the release of AST in the sham renal ischemia experiments (Maessen,1988p). In the present study the need for correction was avoided as all experiments were performed in chronically instrumented, conscious dogs. In these experiments no $\mathrm{CK}$ release was observed.

Changes in plasma enzyme activity following ischemic renal tissue damage have been considered small and rather undefined and hence of limited diagnostic importance (Price,1982;Ringoir,1968). The present data show that at $24 \mathrm{hrs}$ following $90 \mathrm{~min}$ of ischemia the plasma LDH and AST activity is indeed moderately increased and has been normalized within 48 hrs suggesting only temporarily increases of plasma enzyme activity. However, frequent measurements of the plasma enzyme activity immediately following the onset of reperfusion reveals a rapid and marked rise within one hour which reaches peak values between 4 and 8 hrs exceeding normal values 4 to 10 -fold. Following perma- 
nent ischemia a similar albeit delayed release pattern is observed. So the appearance of renal enzymes in the circulation is characterized by a rapid and very temporarily release which will only be found by early and frequent sampling following the onset of ischemia and reperfusion.In this way a reliable calculation of the cumulative amount of enzyme activity in the circulation following permanent ischemia as well as during reperfusion after transient ischemia is possible.

Within the range of error due to methodological limitations and interindividual variability the percentage of AST recovery found in this experimental study is satisfactorily. The LDH recovery, however, shows some unexpected errors that cannot be readily explained. It is presently investigated whether these errors can be explained by more detailed $\mathrm{LDH}$-isoenzyme analysis.

It may seem rather surprising that the permanently ischemically damaged tissue does not show a markably larger enzyme loss than the tissue reperfused after 90 minutes of ischemia. A possible explanation might be that the enzymes liberated in the infarcted area are unable to reach the plasma. In a previous study, however, it was found that enzymes entering the interstitium in the ischemic tissue are allmost immediately transported to the plasma (Maessen,1987b). In the present study it was furthermore shown that after 90 minutes of permanent ischemia still no increase in plasma enzyme activity is found suggesting that the cellular membrane integrity is maintained and no enzyme activity is lost. Reperfusion of kidneys following 90 minutes of ischemia, however, induced an immediate and large increase of plasma enzyme activity indicating profound cell damage. These arguments strongly favour the existence of either a sudden cellular injury in addition to the preceding ischemic injury or an acceleration of injurious processes that would otherwise take more time. The existence of such a phenomenon of postischemic reperfusion injury, possibly mediated by radical oxygen metabolite formation, has been suggested previously but is not generally accepted (Kako,1988;Koyama,1985). If cellular enzyme loss reflects cell damage, the present results indicate the occurrence of reperfusion injury.

\section{SUMMARY}

The present study demonstrates that the extent of ischemic damage in kidney as expressed by the loss of tissue enzyme activity can be estimated by measuring plasma enzyme activity in serial blood samples. Renal ischemia was induced in chronically instrumented dogs. The effect of both permanent and transient ischemia was studied. Two days following the ischemic insult the tissue enzyme depletion was determined. Changes in plasma enzyme activity during ischemia and reperfusion were monitored by frequent blood sampling at regular intervals. The cumulative amount of enzyme activity released into plasma during the observation period was calculated according to a two-compartment model for circulating proteins. Thus the cumulative release of enzyme activity in plasma could be expressed as a percentage of the total depletion of tissue enzyme activity. Cytosolic aspartate amino transferase was almost completely recovered. This recovery was found both after permanent and transient ischemia. Only for large injuries, with enzyme depletions exceeding 20 gram equivalents of tissue, the recovery became incomplete. Some unexplained irregularities were observed for lactate dehydrogenase, which was also measured.

Evidence is provided that the sudden, massive release of enzymes in plasma observed 
after postischemic reperfusion is not a washout phenomenon but rather reflects an acceleration or exacerbation of the development of severe cell damage. 


\section{CHAPTER 13}

\section{LOSS OF ENZYMES FROM VIABLE KIDNEY TISSUE ${ }^{1}$}

\section{INTRODUCTION}

In the pathogenesis of ischemic injury the occurrence of cellular membrane disruption is a key feature of irreversible damage (Hochachka, 1986; Jennings, 1985). Loss of membrane integrity is associated with failure of the cellular energy metabolism (Duvall,1986; Venkatachalam,1988). Inadequate ion pumping due to lack of energy supply may lead to the increased cellular entrance of $\mathrm{Ca}^{2+}$ ions which initiate processes leading to membrane lipid abnormalities and membrane disruption (Trump,1981). In myocardial ischemic injury the extent of enzme leakage from cells with disrupted membranes is associated with the extent of irreversible cell damage or "infarct" (Hearse,1979). The release of cellular enzymes into the circulation has been used to assess the extent of necrosis in myocardial infarction (Roberts,1975; Willems,1985). It is unknown, however, whether extensive irreversible cell damage plays a dominant role in the development of prolonged renal failure after ischemic injury to kidneys (Donohoe,1978;Solez,1984). Estimations of renal enzyme release after renal ischemia have recently suggested profound renal tissue necrosis in kidneys that develop severe acute renal failure following ischemic injury (Maessen,1987). It is however not certain that in kidneys the loss of enzymes after ischemia is restricted to irreversible damaged cells, similarly as in heart tissue. For instance in liver injury enzyme leakage, especially of cytosolic enzymes, is not considered to prove irreversible cell damage (Hermens,1982).

According to the role of the energy metabolism in the development of ischemic membrane disruption the depletion of high energy nucleotides should correlate with the loss of enzymes (Jennings,1985). In the present study in canine kidneys changes in adenine nucleotide levels were related to changes in cellular integrity as a result of ischemic injury. The structural integrity of the cells was monitored by assessment of changes in tissue enzyme activity. Cytosolic, mitochondrial, and brush border enzymes were considered. To identify leakage as the cause of cellular enzyme loss the recovery of enzyme was calculated during a period of $48 \mathrm{~h}$. The question was addressed whether the exhaustion of the adenine nucleotide pool matches with the depletion of tissue enzyme activity.

\section{MATERLALS AND METHODS}

Experiments were performed on adult mongrel dogs of both sexes weighing between 18 and $25 \mathrm{~kg}$. Anaesthesia was induced with thiopentotal ( $20 \mathrm{mg}$ per $\mathrm{kg}$ body weight) and maintained with a nitrous oxide-oxygen gas mixture, delivered continuously by a positive pressure respirator via an endotracheal tube. During surgery animals were placed on a thermostatic watermattress. Animals were fasted during $24 \mathrm{~h}$ before surgery with free access to water. In a first series of experiments renal ischemia was induced in anaesthetized animals. A midline abdominal incision was made and the vessel pedicle of the left kidney was clamped for either 30 or 90 minutes. Before removal of the clamp a contralateral nephrectomy was performed. Animals were allowed to recover before 
removal of the left kidney after 12,24 , or $48 \mathrm{~h}$ of reperfusion. In sham-operated animals whose kidneys were not clamped, the right kidney was removed immediately and the left kidney after $48 \mathrm{~h}$. In a second series of experiments renal ischemia was induced in conscious dogs. Six days before the induction of renal ischemia a mechanical occluder device was mounted onto the left renal artery. A polyethylene catheter was secured in the jugular vein. Ischemia was induced for either 30 or 90 minutes. The left kidney was removed after $48 \mathrm{~h}$. Additionally, in a number of animals an occluder device was mounted on the left main branch of the renal artery to induce permanent occlusion of this vessel and hence, permanent ischemia of about one half of the kidney. The effect of the occlusion was checked before removal of the kidneys after $48 \mathrm{~h}$ by the infusion of a large amount of Evans blue into the aorta. Measurement of adenine nucleotides In the first series of experiments, biopsies were harvested from renal cortex, using a surgical blade technique which has been described previously, for determination of adenine nucleotide levels (Maessen,1988a). Biopsies were taken before and after ischemia, during reperfusion after 5 and 60 minutes, and before the kidneys were removed either after 12 , 24 , or $48 \mathrm{~h}$. The biopsies were immediately frozen in liquid nitrogen and then stored at $-80^{\circ} \mathrm{C}$ before analysis. ATP, ADP, and AMP were measured using high-performance liquid chromatography by a modification of the method of Wynants and van Belle (Wynants,1985; Maessen,1988b). Total adenine nucleotide levels were used as a parameter of the energy status of kidneys and were calculated as the sum of ATP, ADP, and AMP (Pegg,1976; Maessen,1988a). Measurement of enzyme activities. In the second series of experiments enzyme studies were performed. For determination of changes in plasma enzyme activity blood samples were obtained one hour before and $0,1,2,4,6,8,12,16$, $20,24,30,36,42$, and $48 \mathrm{~h}$ after the onset of ischemia. In a previous study we have shown that the cumulative amount of AST entering the blood circulation reflects the loss of AST activity from the ischemic kidney (Maessen,1988j). Changes in plasma AST activity were therefore used to monitor the loss of renal enzyme activity in time. The cumulative loss of enzyme activity into the circulation during a period of time was calculated according to a two-compartment model for circulating proteins as described before (Hermens, 1982). Briefly: The cumulative amount of enzyme released up to time t per litre of plasma, $Q(t)$, is calculated as $Q(t)=C(t)+E(t)+F C R_{o} \int^{t} C(t) d t$ in which $C(t)$ presents the enzyme activity in plasma (corrected for the steady state activity) and $\mathrm{E}(\mathrm{t})$ the extravascular enzyme activity, both at time t. The last term gives the enzyme activity which has been eliminated from plasma up to time t (FCR is the fractional catabolio rate constant). The extravascular enzyme activity is calculated as: $E(t)=$ TER.exp(-ERR.t) of ${ }^{t} \exp (E R R . t) C(t) d t$ in which TER (transcapillary escape rate constant), ERR (extravascular return rate constant), and FCR (fractional catabolic rate constant) have been determined in the dog in previous studies (Hermens, 1982). Values of TER $=0.032 \mathrm{~h}^{-1}$, ERR $=0.068 \mathrm{~h}^{-1}$, and FCR-AST $=0.21 \mathrm{~h}^{-1}$ were used. Total release of enzyme activity was calculated by multiplication of $Q(t)$ with the plasma volume of the individual animal calculated from body weight $(47 \mathrm{ml} / \mathrm{kg})$. For the determination of changes in renal enzyme content the kidneys were dissected, and divided in samples of 5-6 g wet weight. These samples were completely homogenized at $4^{\circ} \mathrm{C}$ with an ultra-turrax in $15 \mathrm{ml}$ of Tris buffer $0.05 \mathrm{M}, \mathrm{pH} 8.5$ containing $0.05 \mathrm{M} \mathrm{NaCl}$ and $0.015 \mathrm{mM}$ pyridoxal phosphate, and subsequently ultrasonificated. Errors introduced by changes in tissue water content were corrected by calculation of tissue dry weight. A panel of enzyme activities were con- 
sidered including lactate dehydrogenase (LDH, EC 1.1.1.27), aspartate aminotransferase (AST, EC 2.6.1.1), glutamate dehydrogenase $(\mathrm{GLDH}, \mathrm{EC}$ 1.4.1.2.), alkaline phosphatase ( $A P, E C$ 3.1.3.1.), and gamma glutamyltransferase (GGT, EC 2.3.2.2.). Enzyme activities were assayed spectrophotometrically at $25^{\circ} \mathrm{C}$ using commercially available test kits (Boehringer, Mannheim, FRG) and a Cobas Bio automatic analyzer (Hoffmann-LaRoche, Basle, Switserland).

\section{RESULTS}

\section{Adenine nucleotides}

Adenine nucleotide levels decreased along with ischemia time (Figure 13.1 and 13.2). During reperfusion the restoration of adenine nucleotides levels in kidneys after $30 \mathrm{~min}$ of ischemia started within 5 min reaching $80 \%$ of control values after $60 \mathrm{~min}$. In severely damaged kidneys the recovery was much more delayed. However, within $24 \mathrm{~h}$ concentrations close to control were obtained. After $48 \mathrm{~h}$ reperfusion adenine nucleotide levels

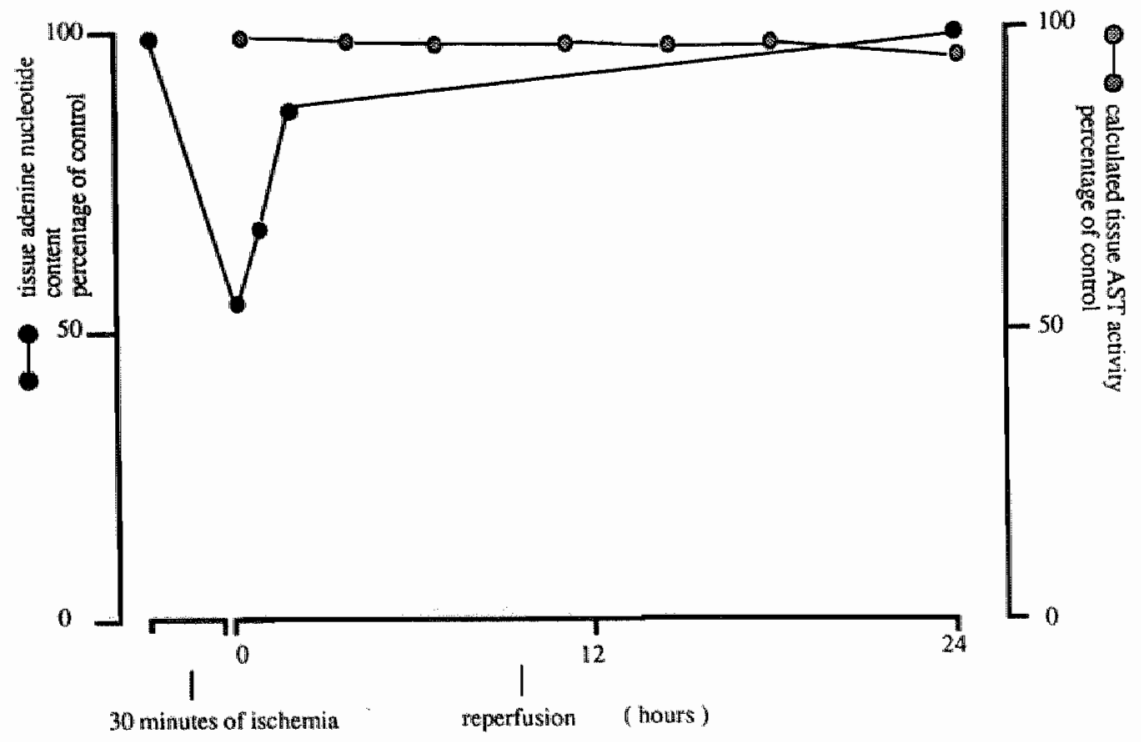

Figure 13.1a: Time course of changes in the renal adenine nucleotide content and the cumulative release of AST in plasma. The cumulative AST activity is expressed as a percentage of the tissue AST activity of control kidneys. All data points represent mean values of six experiments. 


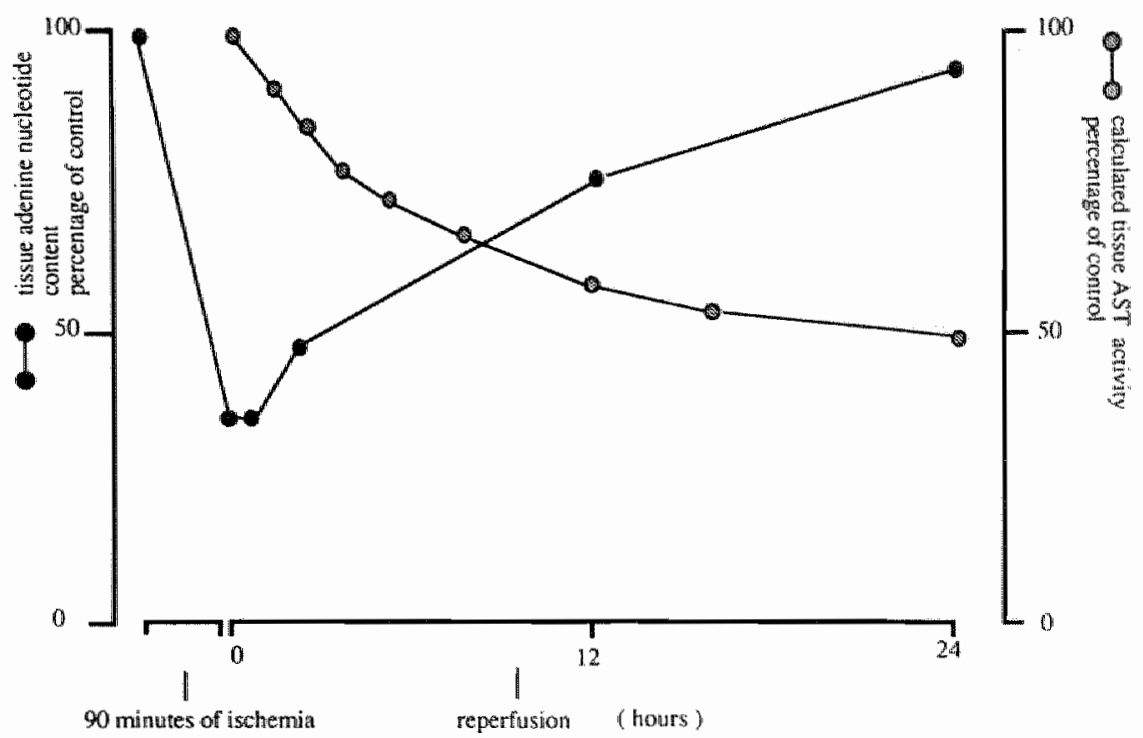

Figure 13.1b: Time course of changes in the renal adenine nucleotide content and the cumulative release of AST in plasma. The cumulative AST activity is expressed as a percentage of the tissue AST activity of control kidneys. All data points represent mean values of six experiments.

were not significantly different from values after $24 \mathrm{~h}$.

Renal tissue enzyme activity

Table 13.1 summarizes the changes in renal tissue enzyme content in various experimental groups. It is apparent that the reduction of renal mass due to contralateral nephrectomy results in a large increase of the brush border enzymes, AP and GGT, and similar but smaller increases in LDH, AST, and GLDH within 48 hours. After 30 minutes of ischemia and $48 \mathrm{~h}$ reperfusion no significant depletion of renal tissue enzyme activity was observed. After 90 minutes of ischemia and $48 \mathrm{~h}$ reperfusion, however, the cytosolic and mitochondrial enzymes (LDH, AST, and GLDH) was reduced to about $55 \%$ of preischemic control. In renal tissue with permanent arterial obstruction, the depletion was similar (LDH) or more severely (AST, and GLDH). One third of the mitochondrial GLDH activity was left in this tissue. The pattern of changes in the activity of the brush border enzymes was different. Both after 30 and 90 minutes of ischemia an increase rather than a decrease of AP was found. Such an increase did not occur in permanently ischemic tissue. GGT values were not significantly different from control in either of these groups.

There was a sharp difference between leakage of enzyme from kidneys after 30 and 90 
$\mathrm{min}$. In the first group of kidneys the release of enzyme activity in plasma was negligible whereas in the latter group of kidneys almost half of the total renal AST content was found in plasma within 24 hours. These data were in agreement with the findings on the AST tissue activity. The largest proportion of enzyme was lost during the first six hours after reperfusion. Comparison of the changes in adenine nucleotides and the AST activity demonstrated a remarkable discrepancy of the tissue depletion after $24 \mathrm{~h}$. The de-

\begin{tabular}{|c|c|c|c|c|c|c|}
\hline Wowo & $(n)$ & L助县 & 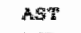 & GLOHA & $A F$ & GET \\
\hline $\begin{array}{l}\text { control } \\
\text { a lues }\end{array}$ & 10 & $225+110$ & $45 \pm 1$ & $4 z \pm 2$ & $21 \pm 1$ & $36 \div 3$ \\
\hline 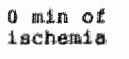 & 6 & 25.20 & $57 \pm 1^{\mathrm{B}}$ & $65 \pm 1^{12}$ & $5 \mathrm{l} \pm 2^{2}$ & $54 \pi 4^{2}$ \\
\hline 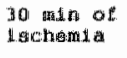 & 5 & 206422 & $44 \times 3$ & 40 年 3 & $35 \pm 0^{2}$ & $37 \pm 4$ \\
\hline $\begin{array}{l}90 \text { min } o f \\
13 \text { clisemita }\end{array}$ & 6 & $129+11^{2}$ & $25 \pm 2^{a}$ & $243^{\mathbf{a}}$ & $2 \theta \pm 2^{a}$ & $24+1^{2}$ \\
\hline $\begin{array}{l}\text { peraratert } \\
\text { lochand }\end{array}$ & 5 & $110 \times 10^{2}$ & $d \pi \pm z^{\pi}$ & $14+2^{2}$ & $12 \pm 2^{3}$ & $21 \div 4^{2}$ \\
\hline
\end{tabular}

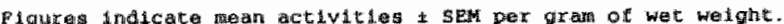
Q3ignitlcant different from cented (p<0.05) as determined with sudent" to-cest.

layed nucleotide recovery corresponded to the profound enzyme leakage in kidneys with 90 min of ischemia.

\section{DISCUSSION}

Comparison of adenine nucleotide depletion and enzyme depletion in the development of irreversible damage in severe ischemic kidneys, produces some unexpected observations. After an initially depletion the adenine nucleotides rapidly recovered after reperfusion to near normal values within 24 hours. Since the synthesis of high energy nucleotides is only possible at a high energy cost with intact mitochondria these findings suggest that the majority of cells remained viable (Hochachka,1986). By contrast, in the same experimental model the renal tissue activity of both cytosolic and mitochondrial enzymes was remarkably reduced to about 50 to $60 \%$ of control within 48 hours of reperfusion. Loss of macro-molecules from ischemically injured tissues has been regarded as a symptom of cell death (Reimer,1984). Thus, the loss of tissue enzyme activity suggests that almost $50 \%$ of the renal cells were non-viable. The conflicting information obtained with both these parameters is pertinent to a general discussion on the correlation of structural cellular damage and the development of postischemic failure in the kidney (Solez,1985). Two explanations can be hypothesized to bring both observations in one concept. First, the changes of the tissue adenine nucleotide content upon reperfusion are an effect of compensatory mechanisms in a limited number of non-irreversible damaged cells. The remaining cells died and lost their cellular content to the environment. Second, the ischemic injury leads to a transient increase of membrane permeability in a large number of cells which results in the loss of macro-molecules, i.c. enzymes. The rapid recovery of the energy metabolism demonstrated the maintenance of cellular via- 
bility. With respect to the first explanation the occurrence of compensatory, supranormal nucleotide levels has to be assumed. The second explanation is only justifiable if viable cells can loose a considerable part of their enzyme content.

Compensatory changes in metabolic properties following reduction of renal mass as a result of ischemic injury or by ablation are a well recognized phenomenon in the kidney (Favre,1981). Four weeks after two-third reduction of renal mass of a remaining kidney in a unilateral model in rat, the metabolic activity in a tubular cell is approximately increased threefold as assessed by oxygen consumption (Harris,1988). The fundamental question whether renal cells are able to maintain supra normal levels of high energy phosphates has been studied in isolated rabbit kidney tubules. Exogenous administration of ATP, ADP, and AMP can induce a two to threefold increase of cellular ATP, Smaller effects below the level of normal have been obtained in previously ischemically injured tubules. However, since no beneficial effect of manipulating the intracellular ATP levels on the postischemic metabolic integrity and viability of the tubules was observed, the importance of this phenomenon in vivo is not clear (Weinberg,1986). An improved recovery of ischemically depleted nucleotide stores has been achieved in canine kidneys with a number of pharmacological agents. The eventual nucleotide level, however, was always below or equal to normal (Garvin,1985). To the best of our knowledge no supranormal nucleotide levels as a result of endogenous nucleotide synthesis have been reported. Stimulation of renal work load by hypertonic saline loading does not increase renal nucleotide levels (Urbaitis,1984). Similarly, in the present study no significant increase above control values was observed in kidneys with increased work load after $48 \mathrm{~h}$ of contralateral nephrectomy. According to these data it is highly unlikely that the rapid recovery of nucleotides in kidneys after 90 minutes of ischemia as found in the present study was induced by compensatory nucleotide synthesis in a limited number of cells. Alternatively, an increase in nucleotide levels can be explained by an increase in the number of cells either as a result of compensatory growth or as a result of infiltrating inflammatory cells. With respect to the first, a rapid and early increase in the number of cells is unlikely since compensatory growth has been demonstrated to be dominated by hypertrophy rather than by hyperplasy (Fine,1987). A substantial contribution of infiltrating cells is also doubtful. Neutrophils have a characteristic high LDH content in comparison to their AST content (Hollaar,1978). A quantitatively important amount of invaded neutrophils would change the normal LDH/AST ratio of the renal tissue. Such a shift was not observed in the present study.

Profound leakage of macro-molecules from ischemic cells has been assumed to result from membrane disruption and is hence considered to indicate cell death. The lost enzyme activity can be quantitatively determined in plasma (Hermens, 1982). Enzymes have therefore been used to assess infarct size in myocardial infarction (Roberts,1975; Willems,1985). A number of recent clinical studies have supported this concept (Willems,1987). In vitro studies on rat heart cells, endothelial cells, and renal tubular segments provided further supportive evidence as leakage of enzyme in response to injury correlated with other markers of cell death (Chopra,1987; van der Laarse,1979; Jackson,1984; Levin,1987).

In the present study changes in tissue enzyme activity were monitored in kidneys 48 hours after contralateral nephrectomy and ischemic injury. No significant decrease was observed in kidneys after $30 \mathrm{~min}$ of ischemia. Since the recovery of AST in plasma was neg- 
ligible it is amenable that the tissue activity after $48 \mathrm{~h}$ reflected the absence of enzyme leakage rather than the combination of depletion and subsequent normalization by an increase of enzyme activity. In contrast, in kidneys after prolonged ischemia (90 min) and permanent ischemia, the cytosolic and mitochondrial enzyme activity was remarkably reduced. Calculation of the cumulative amount of AST that appeared in the circulation after renal ischemia for $90 \mathrm{~min}$ demonstrated that the loss of tissue enzyme activity was attributable to enzyme leakage into the environment. It has been demonstrated previously that kidneys after $90 \mathrm{~min}$ of ischemia recover functionally after about 5 weeks whereas the permanently ischemic kidneys eventually turn into scar tissue (Maessen, 1988॥, and unpublished observation).

The increase of enzyme activity in non-ischemically injured kidneys following contralateral nephrectomy suggested that within $48 \mathrm{~h}$ a compensatory reaction to a reduction in renal mass can be expected. Similarly, in kidneys with 30 minutes and even 90 min of ischemia, an increase of alkaline phosphatase activity was observed. In contrast, alkaline phosphatase activity in the permanent ischemic tissue decreased similarly to the profound depletion of the cytosolic and mitochondrial enzymes.

Theoretically the ischemic renal tissue may be considered as a mixed cell population in which various types of cells can be discriminated. A number of cells may get irreversible damaged during the ischemic interval. The cellular content is washed out and there is no recovery of the energy store. A second part of cells may die only some time after the onset of reperfusion as a result of complications secondary to the ischemic insult. Such cells may in part be removed by phagocytosis (Duvall,1986). Other cells maintain viability. After a depletion of adenine nucleotides during the ischemic interval the energy pool is replenished following reperfusion. Possible membrane damage which becomes evident during reperfusion will get repaired (Paddock,1981). In addition, cells may show compensatory reaction in response to the reduction in renal mass. Based on the results of the present study one might argue that in kidneys after 90 minutes of ischemia the latter two types of cells are most prominently present. The rapid increase of nucleotide levels is in agreement with the idea that hyperplasy does not play a major role early during the recovery phase. The almost complete recovery of the cellular energy metabolism is pertinent to findings in a previous study that kidneys after $90 \mathrm{~min}$ of ischemia remain viable and are able to regain life sustaining function after delayed contralateral nephrectomy (Maessen,1987). The increase in alkaline phosphatase activity adds further evidence that the majority of cells was only reversibly damaged and able to compensate. The concomitant remarkable depletion of the cytosolic and mitochondrial enzyme activity, however, indicates that membrane damage occurred whereas the integrity of the cellular energy metabolism was maintained. It is therefore suggested that renal cells may loose part of their enzyme content without being destined to die. 


\section{CHAPTER 14}

\section{INTERLEUKIN 1 AND INTERFERON GAMMA MEDIATED CYTOTOXICITY OF KIDNEY TUBULAR CELLS ${ }^{1}$}

\section{INTRODUCTION}

The effector mechanisms responsible for the inflammatory destruction of tissues during rejection are still poorly understood. Cytotoxic Tlymphocytes (CTL)(Lowry,1987), antibody-mediated lysis via complement or leukocytes, and delayed type of hypersensitivity (DTH) reactions, cytokine mediated processes, have been thought to be involved (Loveland, 1987). Indications for local release of cytokines have been obtained (Lowry, 1987; Ruers, 1987), but the role of cytokines in the rejection process leading to failure of graft function and final tissue destruction is, as yet, unknown. Since the cytotoxic and cytolytic potency of cytokines such as Interleukin 1 (III) and Interferon-gamma (IFN- $\gamma$ ) have been convincingly demonstrated in both tumour cells and micro-organisms (Onozaki, 1985; Bhardway, 1986) the hypothesis was raised that, under certain conditions, they may exert a similar effect on normal parenchyma cells. The data presented here show that II1 and IFN-y may act in concert to induce disruption of the cellular integrity of kidney epithelial cells in vitro.

\section{MATERLALS AND METHODS}

Materials.

Recombinant (r) Ill- $\alpha$ and Il1- $\beta$ were kindly provided to us by Dr. S. Gillis (Immunex, Seattle, WA). rIFN- $\gamma$ was donated by Hoffman LaRoche, Basel, Switzerland).

Cell cultures. The kidney epithelial cells were obtained by trypsinization of small biopsies of human kidneys harvested for organ transplantation which could not be grafted for technical reasons. The cells were cultured in Dulbecco's MEM in the presence of $20 \%$ foetal calf serum and antibiotics. For all experiments, the cells were cultured on gelatin coated surfaces in the presence of $2.5 \%$ foetal calf serum. The epithelial identity of the cells was established with anti-cytokeratin staining (data not shown). Cells used for the experiments were of 4-6 passage. For microscopy, the cells were cultured on tissue culture slide chambers (Lab-tek Miles, Naperville, IL).

Measurement of protein synthesis.

Cells were cultured as described in cluster well plates (Greiner, Nurtingen, Federal Republic of Germany) and incubated with ${ }^{3} \mathrm{H}$-Tyrosine $(5 \mu \mathrm{Ci} /$ well; specific activity 30 $\mathrm{Ci} / \mathrm{mMol}$, Amersham, United Kingdom) for $4 \mathrm{hr}$. Subsequently, the cells were washed extensively with medium and solubelized in Zaponine (a detergent, Coulter Electronics, Luton, UK) and ${ }^{3} \mathrm{H}$-Tyrosine was counted in a liquid scintillation counter. Controll experiments revealed that the data obtained with Zaponine solubelized cells did not differ significantly from $10 \%$ trichloroacetic acid precipitate (data not given).

Measurement of cytosolic enzymes. 
To determine the lactate dehydrogenase (LDH) content in the cells, confluent cultures in $25 \mathrm{~cm}^{2}$ culture flasks, Greiner, Nurtingen, FRG were subjected to 3 freeze thaw cycles in the presence of distilled water. The $L D H$ was measured with use of an enzyme kit (Boehringer, Mannheim, FRG). The LDH content of the supernatant of treated and non treated cultures was established of both cell free and cell remnants containing supernatant.

The former and latter after 3 freeze and thaw cycles to free all possible cell bound LDH were largely identical indicating that supernatant did not contain significant amounts of non-disrupted cells. LDH present in culture medium containing $2.5 \% \mathrm{FCS}$ was deduced in all supernatant measurements. $\mathrm{LDH}$ added in medium remained stable (95\% of original activity) for $96 \mathrm{~h}$ at $37^{\circ} \mathrm{C}$.

\section{RESULTS}

Influence of Il1- $\beta$ and IFN- $y$ on morphology of kidney epithelial cells. Primary cultures of human renal epithelial cells were incubated with rIFN- $\gamma$ and rIl1- $\beta$. The addition of rIFN- $\gamma$ to the renal cells influenced the appearance of the cells. They obtained an elongated shape and lost their characteristic epithelial morphology. When rIll- $\beta$ was added to the cells, their appearance became irregular with long extensions. Combined treatment with both rIl1- $\beta$ and rIFN- $\gamma$ dramatically increased the morphological disturbances; moreover, a large number of cells, dependent on the rIll- $\beta$ concentration, lost their detachment to the culture dish and were apparently killed. Figure 14.1 shows the appearance of the renal epithelial cells after 48 - $h r$ treatment with either rIll- $\beta$ or rIFN- $\gamma$ or with the combination of both cytokines and control cultures.

Influence of cytokines on protein synthesis.

In order to investigate the possible cytokine effects on the functioning of the renal cells, cellular protein synthesis was studied by monitoring ${ }^{3} \mathrm{H}$-Tyrosine uptake during cytokine incubation. Treatment of the cells with either rIl1- $\beta$ or rIFN- $\gamma$ resulted in a significant inhibition of protein synthesis as soon as $24 \mathrm{hr}$ after the start of the incubation (Fig. 14.2a). The combined treatment with both cytokines showed a greater inhibition of protein synthesis than the single cytokine treatment, suggesting different mechanisms of inhibition for rll1- $\beta$ and rIFN- $\gamma$. Normalized protein synthesis was observed within 72 hrs when, after 24 hrs of incubation with the cytokines, the cells were further cultured in normal medium (Fig. 14.2B). This demonstrated that the inhibition of protein synthesis is reversible. Incubations with both cytokines for 24 hrs showed a reduced reversibility of the inhibition of protein synthesis. Yet, combined treatment lowered the total number of cells, making comparisons with controls difficult.

Release of cytosolic enzymes.

The cytokine effect on cellular integrity was studied by measuring the cellular handling of the cytosolic enzyme lactate LDH. Starting from $24 \mathrm{hrs}$ following the addition of either rIFN- $\gamma$ or rll1- $\beta$ to the cells, a reduction of total LDH content to $55 \%$ and $63 \%$, respectively, was found, whereas the amount of LDH in the supernatant was only slightly increased compared to controls (Fig. 14.3A). Since the half life of LDH in cultured cells is approximately six days, these findings indicate increased protein turnover (Zavortink, 1979). In cell cultures with simultaneous rIl1- $\beta$ and rIFN- $y$ treatment, the total LDH content was considerably reduced to minimum levels of $14 \%$ vs. control.

In contrast to the experiments with single cytokine treatment with Il1- $\beta$ or IFN- $\gamma$, the 
Figure 14.1.: Effect of treatment with $\mathrm{rLL}-\beta$ (100 LAA.F. units), rIFN- $\gamma(100 \mathrm{VU})$ and simultaneous treatment with rIL1- $\beta$ and IIFN- $\gamma$ on cultured human kidney epithelial cells.

Control cultures were either treated with heat inactivated rLI- $\beta$ and rIFN- $\gamma$ (data not given) or not treated at all (a). Note the irregular shape of the cells at $48 \mathrm{hr}$ after treatment with rIL1- $\beta$ alone (b) in comparison to the elongated cells in IFN- $\gamma$ treated sample (c) and with control cultures (a). The cytotoxic effect of the combination of rIL1- $\beta$ and IFN- $\gamma$ (d) is clearly visible. Note the large areas from which the cells have detached themselves and the cytoplasmic and nucleoplasmic vacuolization. rIFN- $\gamma$ treated cells (c) and control cells (a) show no signs of cytotoxicity.

In IL1- $\beta$ treated cultures, signs of cytotoxicity were sporadically observed. In the combined treatment grotup, IFN- $\gamma$ was added to the cultures $18 \mathrm{hr}$ prior to ILI- $\beta$. Cytokines were also added to the cultures on day $2 \mathrm{in}$ order to maintain a relatively constant level. Cells were fixed with $70 \%$ ethanol and stained with HEE $48 \mathrm{hr}$ after addition of IL $1-\beta$.
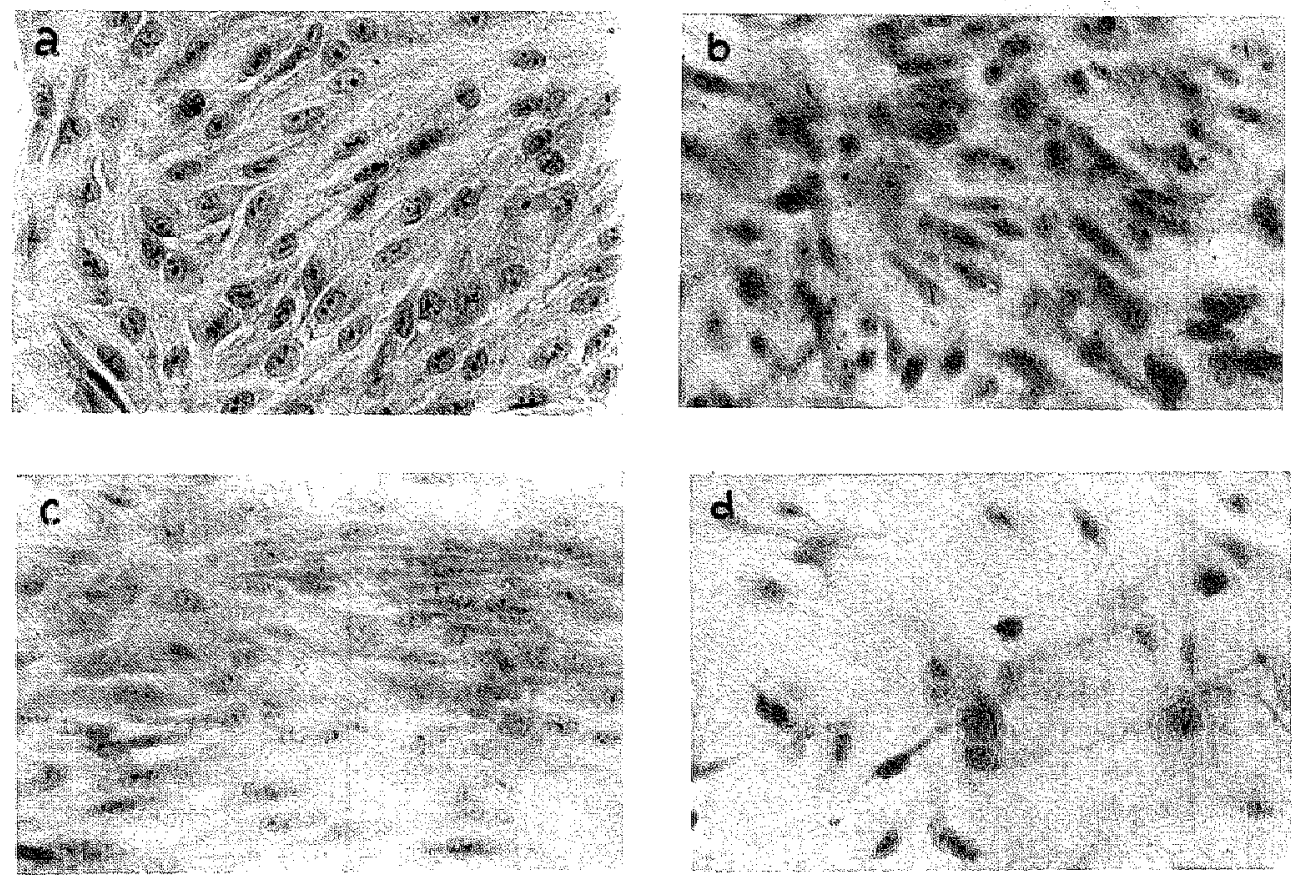

free LDH in the supernatant of cells treated with both cytokines increased to $65 \%$ of total LDH content (Fig. 14.3B). These data are in line with the morphological observations that the combination of rll1- $\beta$ and rIFN- $\gamma$ dramatically induced disruption of the structural integrity of the cells (i.c. induced cell lysis). All experiments mentioned above were per formed with rIl1- $\beta$ comparable results were also obtained with rll1- $\alpha$ (data not given).

\section{DISCUSSION}

The above described influences of rIl1 and rIFN- $\gamma$ in vitro on kidney epithelial cells may very well explain part of the rejection process in vivo. Allograft rejection is known to be 

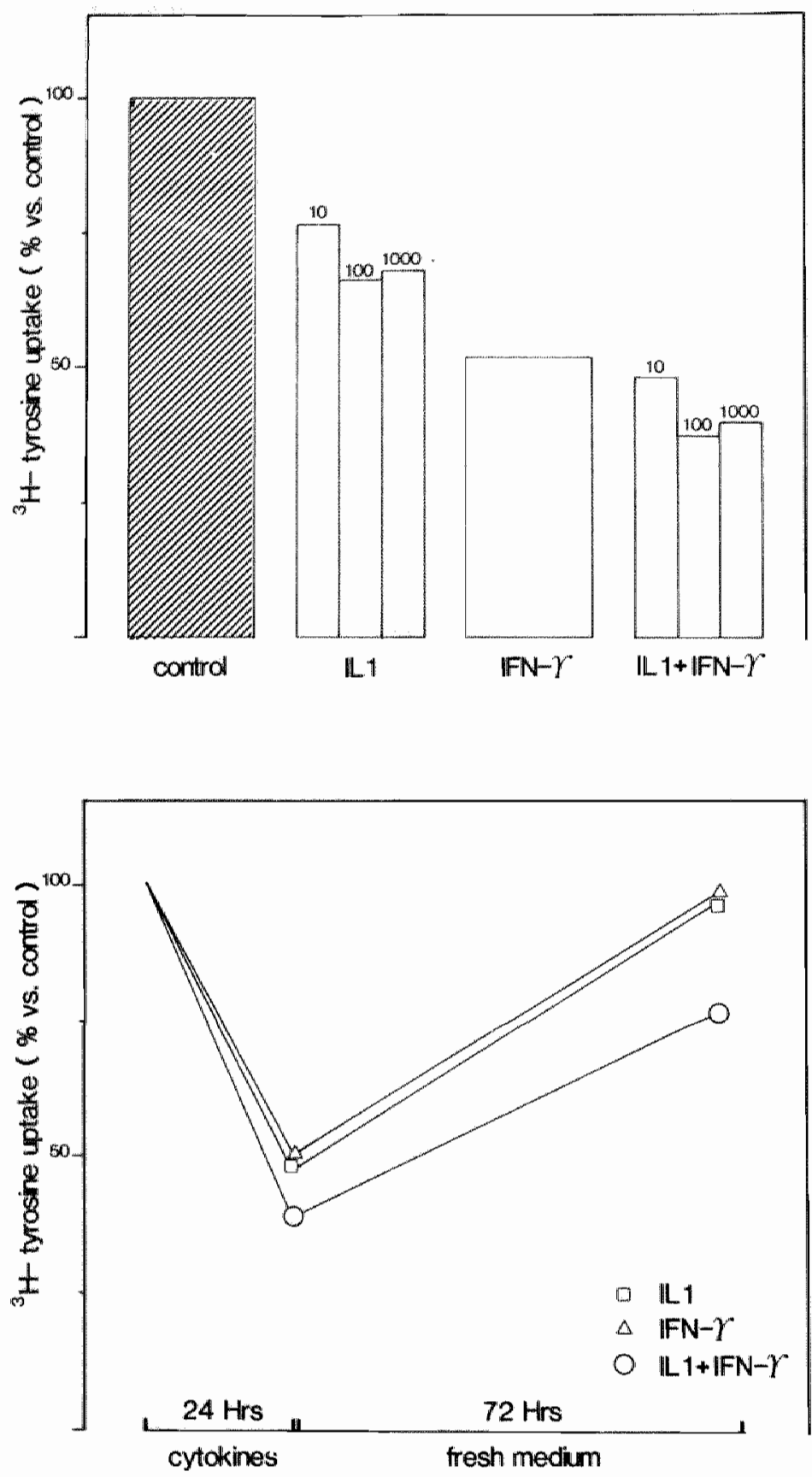

Figure 14.2.: Reduction in protein synthesis induced by cytokines rIL1- $\beta$ and IIFN- $\gamma$. A: The influence of cytokines on ${ }^{3}$ H-Tyrosine uptake as a measure of protein synthesis was determined 24 hr after addition of rlL1- $\beta$ (See figure 14.1].

B: Recovery of protein synthesis was studied following removal of cytokines after $24 \mathrm{hr}$ of treatment. Cells cultured as described above were washed extensively after treatment for $24 \mathrm{~h}$ with cytokines and re-fed with fresh new medjum. ${ }^{3} \mathrm{H}$ Tyrosine uptake was determined after $72 \mathrm{hr}$ culture. Note the almost complete normalization of the ${ }^{3}$ H-Tracing uptake after culture in the ab. sence of cytokines. The data shown are a representative example out of 8 experiments. 


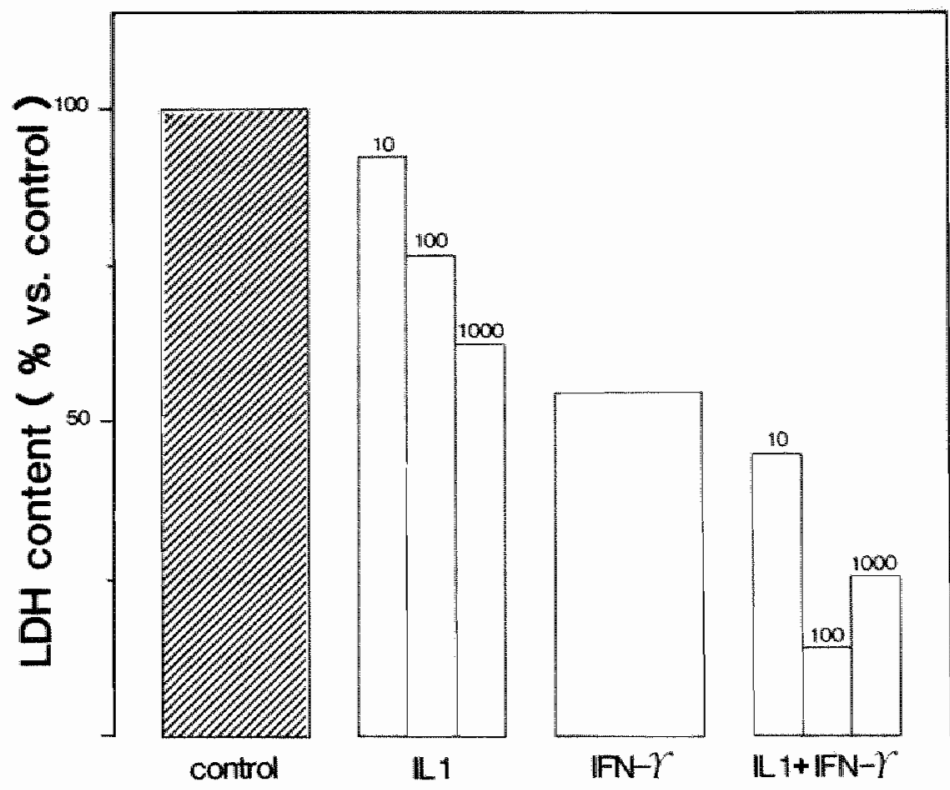

Figure 14.3.: Cytolysis of kianey epithellial cells measured as the loss of the cytosolic enzyme $\mathrm{LDH}$ induced by the toytokines rlL1- $\beta$ and rIFN$\gamma$.A. LDH content of kidney epithellial cells treated with rILL and IIFN-y. Cells were treated as in figure 14.1. The supernatant of the cells cultured on $25 \mathrm{~cm}^{2}$ culture tlasks was harvested $48 \mathrm{hr}$ after addition of ILI. The LDH content in the control cells was $1500 \mathrm{mU} / 10^{6}$ cells. The LDH content of the treated cells plus the LDH content of the supernatant was ex-

pressed as a percentage of the LDH content of the control cultures. Note the reduced LDH content of the cell cultures with cytokines. B. Release of cytosolic LDH by cells treated with the cytokines rlL1 and IFN- $\gamma$. The LDH released by the cells was measured in the supernatant of the above mentioned cultures after the

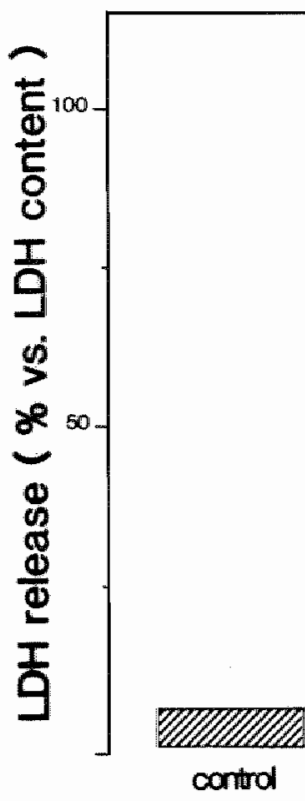

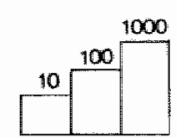

1. 1

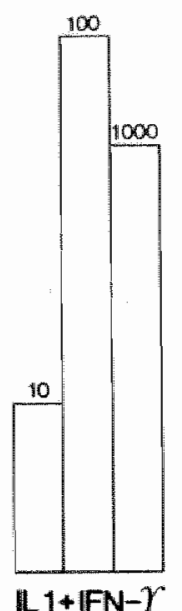
supernatant had been freed of the cell remnants by centrifugation. The amount of $\mathrm{LDH}$ present in the supernantant was expressed as a percentage of the total L.DH content of the corresponding cell cul. tures. Note the increased release of cyto solic LDH by cells subjected to simulta neous treatment with rILI- $\beta$ and IFN $\gamma$. The data shown are a representative experiment of a series of 10 experin ments. 
a complex process in which CTL, DTH, and antibody-mediated type processes play an important role (Loveland,1981; Lowry,1987; Doveren,1986). The mechanism which underlies DTH-induced graft destruction is, as yet, unknown. Inflammatory mediators are reported to be responsible for this process although no proof of lymphokine involvement has thus far been reported (Lowry, 1987). The controversy still exists as to what place CTL-mediated and DTH-mediated destruction have in the process of graft rejection. Macrophages and endothelial cells can produce Il1, a group of cytokines with an extremely wide range of actions (Dinarrello, 1986; Miossec, 1986). It appears that Il1, produced as part of the inflammatory response to injury, infection, or other stimuli, can influence many metabolic, physiologic, and immunologic processes. The ways of action of Il1 can be divided into general effects such as induction of fever or muscle proteolysis (Dinarello, 1985; Dinarello, 1984) and local effects (Gowen, 1986; Bevilaqua, 1984). In vitro data suggest that the local effects largely depend on the type of tissue in which Il1 is released. In bone and cartilage, Il1 inhibits new formation of the matrix (Gowen, 1986). For pancreatic islet cells, it has been shown that 111 has both inhibitory and cytotoxic effects. A key role in the etiology of juvenile diabetes was proposed for IL1- $\alpha$ (Hernanedez,1987). In blood vessels, the hemostasis equilibrium is affected by the induction of procoagulant activity by endothelial cells in response to IL1 (Lehmann,1987) Therefore, it has been suggested that IL1, as a major product of activated macrophages, plays a prominent role in the effector phase of the immune response. The presence of such activated macrophages has been reported in experimental studies in rats showing that tissue injury in renal grafts during rejection occurred after a profound invasion of activated macrophages (Pessina,1987).

The T lymphocyte product IFN- $\gamma$ has been implicated in the process of graft rejection as a mediator responsible for the MHC-class II expression observed during graft rejection (Milton,1985). In a previous study, we showed the presence of IFN- $\gamma$ producing $T$ lymphocytes in rat kidneys during rejection (Ruers,1987). Numerous other IFN- $\gamma$ effects have been identified. IFN- $\gamma$ enhances the antimicrobial activity of both circulating and resident macrophages on invading micro-orgamisms (Bhardway,1986). Recently it was observed in vitro that IFN- $\gamma$ markedly altered the morphological appearance of endothelial cells and inhibited their proliferation (Stolpen,1986). The importance of these observations with regard to in vivo processes remains unknown.

During human renal allograft rejection, eventual destruction is preceded by a period of graft non-function. This functional impairment is reversible since successful anti-rejection therapy leads to return of renal function within a few days (Cosimi,1981). This phenomenon may imply that the impairment of cellular function and cytolysis are two distinct features of the effector arm of the immune response. The data presented here show that two cytokines, rIL1 and rIFN- $\gamma$, can reduce protein synthesis and that combined rIL1 and rIFN- $\gamma$ treatment can cause destruction of normal parenchyma. The former is supposed to be related to the impaired function of rejecting allografted kidneys. The latter is supposed to be possibly related to the process of tissue destruction which is observed in endstage allograft rejection. From a teleological point of view, the fact that two cytokines are necessary to cause strong inhibition of protein synthesis and cytolysis offers a safe regulation of a powerful, destructive process. These data, which are the first to describe the synergistic effect of ILI and IFN- $y$ on non-malignant cells, may, in addition to being of importance to the general knowledge of inflammatory processes, also offer 
new therapeutic modalities for treatment of graft rejection and auto-immune diseases, similarly as reported recently for experimental graft-versus-host disease (Piquet,1987).

\section{SUMMARY}

The effect of II1 and IFN- $y$ on normal human kidney epithelial cells was studied. The data show that human rIl1 and rIFN- $\gamma$ can both alter structural and metabolic processes in primary cultures of normal renal tubular cells. When cells were incubated with the combination of III and rIFN- $\gamma$, however, an increased inhibition of protein synthesis and final destruction of cellular integrity was observed. Hence, it was demonstrated that two cytokines, namely the macrophage product Il1 (both $\alpha$ and $\beta$ ) and the T lymphocyte product IFN- $\gamma$, act in concert to induce disruption of cellular integrity. This phenomenon offers a mechanism for a fine-tuned loss of function and cytolysis of cells in inflammatory tissue which is observed in auto-immune diseases and in graft rejection. 


\section{CHAPTER 15}

\section{ROLE FOR TNF IN THE INFLAMMATORY ENHANCEMENT OF ISCHEMIC RENAL INJURY"}

\section{INTRODUCTION}

The involvement of inflammatory reactions in ischemia-reperfusion associated injury is currently receiving increased attention. Recent studies suggest that the inflammatory response which is elicited by tissue injury may have negative side effects that enhance the eventual damage following an ischemic insult. Prevention of neutrophil infiltration in ischemic tissue reduces infarct size (Romson,1983; Engler,1987). Similarly, inhibition of neutrophil activation has been demonstrated to exert a beneficial effect on postischemic recovery (Farber,1988). However, the actual importance of the inflammatory mechanism as a factor determining the extent of ischemia-reperfusion associated injury has not yet been established.

Recently, it was shown that gram-negative sepsis exacerbates preexisting ischemic renal damage (Zager,1986). The effects of gramnegative septicemia are known to be associated with the induction of extensive inflammatory reactions (Old,1987). We therefore suggest that potentiation of ischemic injury also results from general inflammatory processes others than those elicited by the ischemic tissue injury.

The macrophagederived hormone tumor necrosis factor (TNF) has been shown to play a central role in inflammatory processes such as sepsis (Kettelhut,1987). Treatment with monoclonal antibodies against TNF has been successfully used to inhibit the development of lethal complications as well as damage to organs during gramnegative sepsis (Tracey,1987;Beutler,1985). Therefore, TNF is likely to be a mediator of ischemia-reperfusion associated injury.

Here we investigated whether the enhancement of ischemia-reperfusion associated injury results from a generalized stimulation of inflammatory reactions. It was found that treatment with human recombinant TNF, similar as with endotoxin, can enhance the extent of ischemic renal injury. Furthermore, it is demonstrated that the relation between inflammation and ischemic tissue is more extensive than is generally considered.

\section{MATERIALS AND METHODS}

\section{Animals:}

Male Sprague Dawley rats weighing 200-250 g were obtained from Charles River Breeding Laboratories. They were maintained on a standard laboratory diet and were allowed free access to water.

Reagents

Lipopolysaccharide from Escherichia coli (LPS, 055:B5) was obtained from Sigma (St. Louis,MO) and suspended in sterile pyrogen-free saline. Human recombinant tumor necrosis factor was kindly provided by Cell Tech (Slough,UK). Before use, stock solutions were diluted in pyrogen-free saline.

Surgical protocol 
Animals were anesthetized with ether. Surgery was performed on a temperature controlled watermattress. After making a midline abdominal incision both kidneys were dissected from surrounding tissues. The renal vessel pedicles, including the ureter, were clamped with soft tip, vascular clamps. During the ischemic interval the abdomen was closed. Immediately following removal of the clamps to reinstall renal blood flow, the abdomen was closed. Nephrectomy was performed with one ligature around the vessel pedicle and complete removal of all renal tissue. Blood samples were collected by orbital punction.

\section{Experimental design}

To study the effect of LPS stimulation on renal ischemic damage, kidneys were rendered ischemic during either 30 or 60 minutes. Two injections of LPS were administered in a total dose of $0.75 \mathrm{mg}$ per $100 \mathrm{~g}$ body weight, the first one sub cutaneously just before the release of the vessel clamps and the second one intraperitoneally 30 minutes after the release of the clamps. To examine whether the observed effects were dose dependent, a second series of experiments were performed in which a double dose of LPS (1.5 mg per $100 \mathrm{~g}$ body weight) was administered in animals after 30 minutes of bilateral renal ischemia. The effect of rTNF was studied by injecting a single dose of $110 \mu \mathrm{g}$ per $100 \mathrm{~g}$ body weight in the penile vein prior to the onset of reperfusion after 30 minutes of ischemia. In a first group of control experiments LPS and rTNF injections were administered in the same way as in the other groups, however, without clamping of the renal vessel pedicle. In a second control group LPS injections were given in animals without renal ischemia but after bilateral nephrectomy. All injections were given in a volume of $1.0 \mathrm{ml}$. Blood samples for measurement of circulating TNF levels were taken before surgery and 1,2 ,and 4 hours after surgery. Blood urea nitrogen levels were determined after 24 and 48 hours.

Assay

TNF activity in plasma was determined in a biological assay based on L929 cells as described before (Ruff,1981). TNF values were calculated based on the values of a standard serial dilution of human recombinant TNF and expressed in pg per ml.

Data analysis

All values are means $\pm \mathrm{SE}$ of 6 or 7 experiments. Statistical analysis was performed with the Wilcoxon ranksum test or the Mann-Whitney $U$ test. P values less than 0.05 were considered significant.

\section{RESULTS}

\section{LPS treatment}

Animals received LPS either or not in combination with ischemic renal injury. When animals were injected with $0.75 \mathrm{mg}$ LPS $/ 100 \mathrm{~g}$ no significant increase of the blood urea nitrogen level was observed. Doubling of this dose resulted in a small increase at 24 and 48 hours (table 15.1). The latter group of animals showed moderate signs of illness (lethargy, rough fur). Lethality was not observed (figure 15.1). Blood urea nitrogen levels in animals with bilateral renal ischemia ( 30 minutes) doubled during the first 24 hours of reperfusion but returned almost completely to normal within 48 hours. Simultaneous treatment of these rats with LPS $(0.75 \mathrm{mg})$ induced a significant further increase of the blood urea nitrogen levels both after 24 and 48 hours. The high dose of LPS (1.5 mg) in combination with renal ischemia ( $30 \mathrm{~min}$ ) caused a sharp increase of BUN similarly as 
observed after bilaterall nephrectomy after 24 hours. During the next 24 hours only a

A

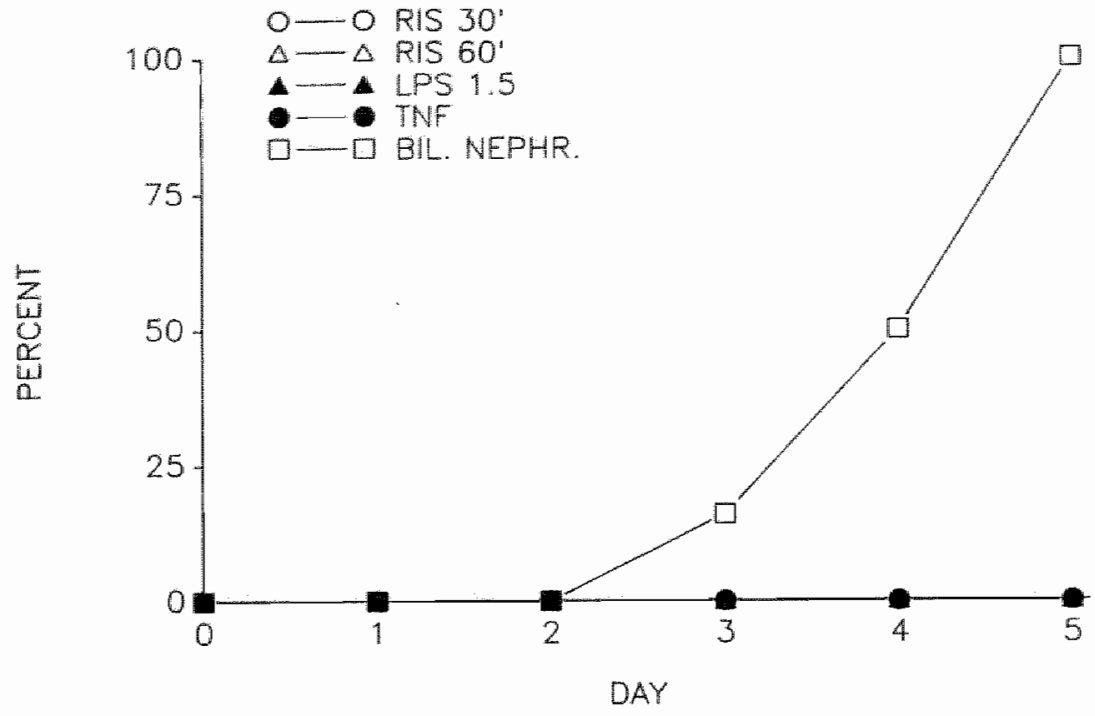

Figure 15.1.: A. Cumulative mortality of rats following renal ischemia , bilateral nephrectomy, LPS injections, or recombinant TNF injections. Only bilateral nephrectomy induced lethality.

B. Cumulative mortality of rats following bilateral renal ischemia combined with endotoxin or TNF.

B

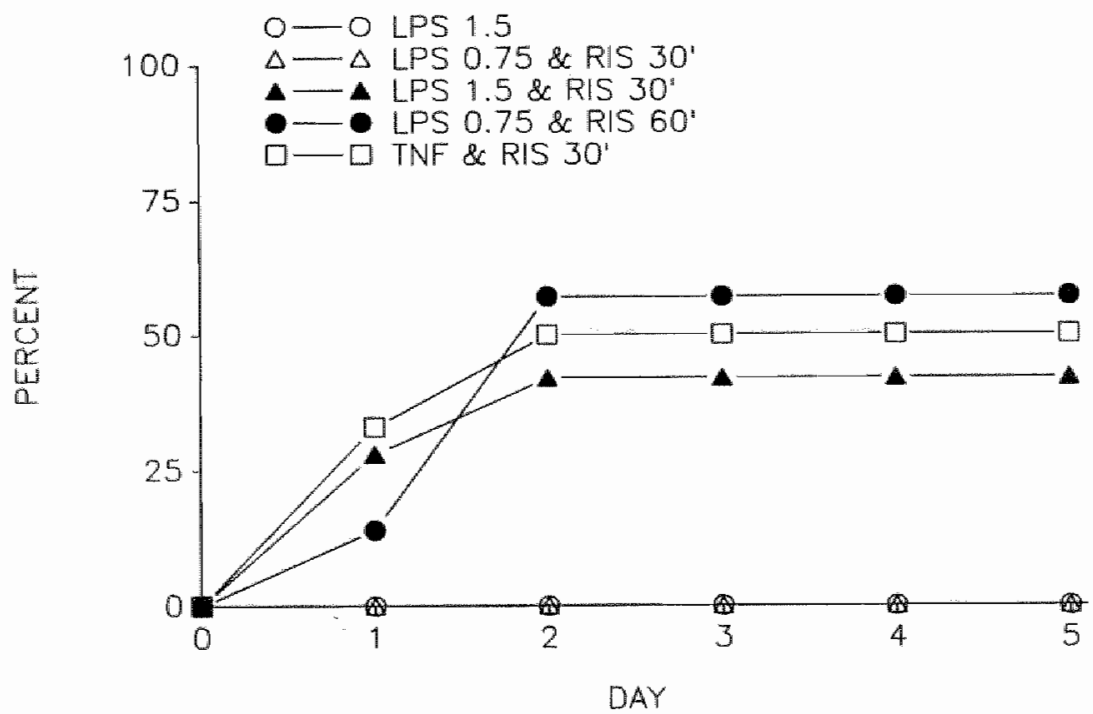

small reduction of the increased blood urea nitrogen levels was

observed. The animals in this group with 30 minutes of ischemia and $1.50 \mathrm{mg} / 100 \mathrm{~g}$ body weight LPS, were severely ill with symptoms of diarrhoea and blood stained fluid exudation around the eyes. Three out of seven animals died within 48 hours. When animals were subjected to 60 minutes of billateral renal ischemia similar results were obtained at 


\begin{tabular}{|c|c|c|}
\hline Group & $24 \mathrm{~h}$ & 48 \\
\hline controll & $7 \pm 0.2$ & $7 \pm 0.1$ \\
\hline 1.0 .75 & $8 \pm 0.4$ & \pm 0.5 \\
\hline LPS 1.50 & $10 \pm 0.3$ & $9 \pm 0.5$ \\
\hline RIS $30 "$ & $15 \pm 0.3$ & $\because \pm 0.2$ \\
\hline RIS $30^{\circ}+$ LPG 0.75 & $24 \pm 1.5$ & $14 \pm 1.4$ \\
\hline PIS $30^{\circ}+$ LPS 1.50 & $50 \pm 4.9$ & $41 \pm 10$ \\
\hline RIS $60^{\circ}$ & $4 \pm \pm 4.0$ & $41 * 4.2$ \\
\hline FIIS $60^{\circ}+$ LPS 0.75 & $57 \pm 5.1$ & $5 \pi$ t 19.3 \\
\hline TNE & $0 \pm 0.2$ & 70.3 \\
\hline FIS $30^{\circ} *$ TNF & $47 \pm 4.3$ & $30 \pm 3.9$ \\
\hline BHitat. Mephrectomy & $67 \pm 2.0$ & $113 * 2.3$ \\
\hline
\end{tabular}

Abbreviatlons are: RIS, bllateral renal 1 sehemia

the low dose of LPS. Blood urea nitrogen levels were almost maximally raised and four out of seven animals died within 48 hours. Sixty minutes of ischemia without LPS treatment did not induce lethality. Notably, following bilateral nephrectomy most animals died between 48 and 120 hours.

TNF production

When animals were injected with LPS high levels of circulating plasma TNF were found one hour after injection (figure 15.2). Peak values were reached after two hours. TNF levels gradually dropped to reach nadir levels within six hours (data not shown). This

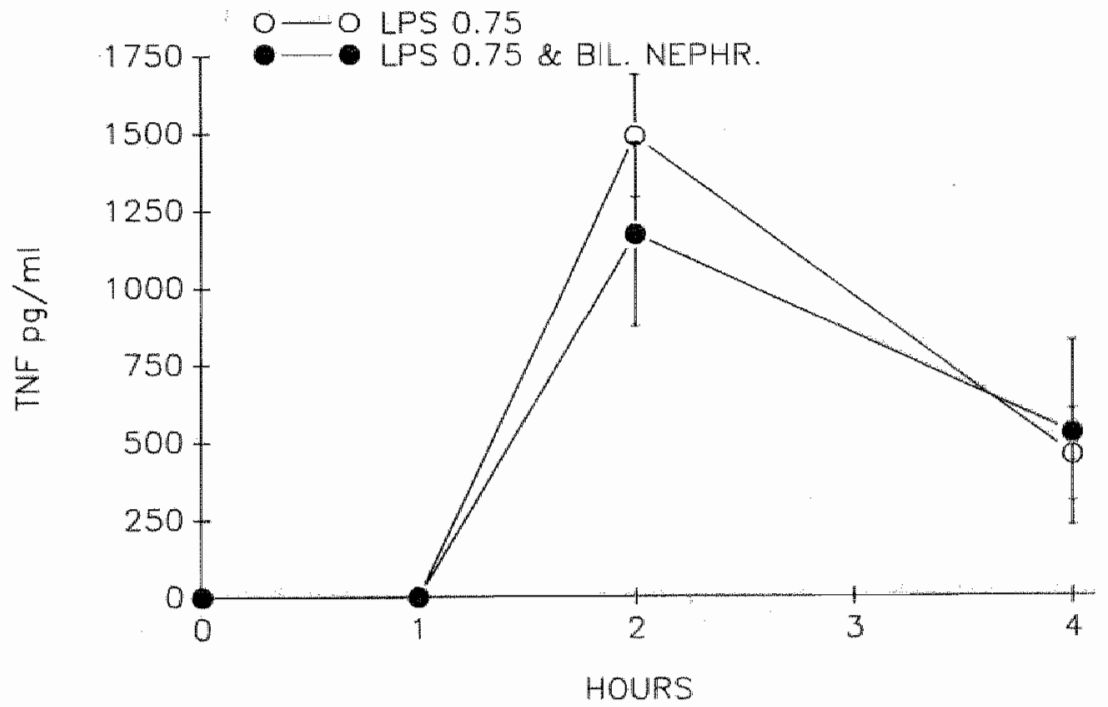

Figure 15.2.: Plasma TNF levels in response to LPS treatment in normal tats and in bilaterally nephrectomized rats.

pattern did not significantly change if before LPS treatment, a bilateral nephrectomy was performed.

Human recombinant TNF treatment 
The combination of 30 minutes of bilateral renal ischemia and recombinant TNF treatment $(110 \mu \mathrm{g} / 100 \mathrm{~g})$ induced a profound increase in blood urea nitrogen levels which was significantly higher in comparison to the increase following single treatment (table 15.1). The increase was also significantly higher than the sum of the increases that were obtained with single treatment. In the combined treatment group three out of six animals died within 48 hours. The other animals survived definitively. After single recombinant TNF treatment, none of the animals died and only moderate symptoms of illness were observed (figure 15.1 and 15,2).

\section{DISCUSSION}

The eventual extent of organ damage that results from an ischemic insult is in part determined by inflammatory processes which are elicited by tissue injury (Henson, 1987). The impact of the inflammatory response is currently reconsidered as recent studies demonstrated a remarkable reduction of the extent of ischemic injury with therapeutic interventions directed against inflammatory reactions. Particularly inhibition of neutrophil involvement has been found to be successful. Depletion of circulating neutrophils can significantly reduce myocardial infarct size (Romson,1983;Engler,1987). Prevention of neutrophil activation with monoclonal antibodies directed against a neutrophil adherence molecule also markedly attenuates the extent of organ injury following haemorrhagic shock (Vedder,1988). On the effector side of the response, blocking neutrophil production of reactive oxygen metabolites has been successful, leading to a profound reduction of postischemic reperfusion injury (Farber,1988;Hernandez,1987). These data provide indirect evidence that the inflammatory response has an important role among factors that determine the extent of ischemia-reperfusion associated injury.

In view of this apparent relationship, the role of the inflammatory response might be considered in a rather different way. If it is shown that depression of the inflammatory response limits the extent of ischemic damage, it is conceivable that, by contrast, stimulation of inflammatory mechanisms exacerbates the native effect of ischemia. This is more likely as it has been demonstrated that the response of the inflammatory system is regulated by the severity of the initial ischemic injury (Engler,1987). Pertinent to this assumption are findings in the present study as well as in previous studies (Zager,1986) suggesting that renal ischemic injury is exacerbated by systemic stimulation with LPS. Following occlusion of renal vessels during 30 minutes the development of renal failure associated with increased blood urea nitrogen levels was markedly enhanced by simultaneous injections of LPS, in a dose-dependent manner. LPS treatment without renal vessel occlusion did not affect renal function. Previously, it has been shown that this treatment does meither negatively influence renal blood flow nor the mean arterial pressure making it unlikely that the observed effect results from sustained ischemia by shock (Zager,1986). These observations may be related to a well-known clinical phenomenon in patients with endotoxemia. Gram-negative sepsis may be complicated by severe acute renal failure which cannot be satisfactorily explained with a relatively mild hypotension. From the present data it is suggested that stimulation of inflammatory mechanisms e.g. by endotoxemia, leads to prolonged ischemic renal failure.

A second dimension to the relationship of inflammation and ischemic tissue injury is sug. gested by other findings in the present study. Following 60 minutes of renal vessel occlusion, leading to severe but reversible renal failure, treatment with LPS appeared to 
induce lethality. Animals died between 24 and 72 hours following surgery indicating that their death was unrelated to renal failure since the survival time of bilateral nephrectomized animals was significantly longer. Therefore, it is suggested that the extensive ischemic renal injury aggravates the generalized effect of the LPS treatment.

Recently, possibly related, mechanisms have been reported combining LPS treatment with a second stimulus. Treatment of mice with D-Galactosamine which is a hepatotoxic agent induces a remarkable sensitization to lethal effects of small doses of LPS. Treatment with either of these substances has no effect on the viability of mice (Lehmann, 1987). Recent studies dealing with LPS mediated inflammatory reactions attribute an important intermediate role to TNF. In experimental as well as in clinical studies high levels of circulating TNF have been found in response to endotoxemia (Old,1987). Recently, inhibition of lethal shock following gram-negative sepsis has been established by previous administration of TNF neutralizing antibodies (Tracey,1987). Furthermore, some effects of LPS treatment can be mimicked with TNF treatment (Kettelhut,1987). In this view we examined whether TNF might play a role in the observed combined effect of ischemic tissue injury and LPS stimulation. It was found that the effect of systemic treatment with non-lethal doses of TNF showed important similarities with the effect of LPS treatment. Renal failure due to short term renal vessel occlusion was markably exacerbated. In addition, the synergism led to systemic complications as a number of animals died within 48 hours. These data therefore provided evidence that the actual potentiating effect was independent from the involvement of bacterial endotoxins.

In a study on the kinetics of circulating TNF it has been suggested that the kidneys are involved in the clearance of TNF (Pessina,1987). Hence, one might argue that the interaction of the stimulated inflammatory response and ischemic tissue injury is a rather kidney specific phenomenon since renal failure might lead to accumulation of circulating amounts of TNF. We therefore measured circulating plasma TNF levels following LPS stimulation with and without concomitant renal failure. It was observed, however, that the characteristic changes of plasma TNF levels upon LPS treatment was not influenced by the absence of normal renal function. Apparently, other mechanisms than renal function were responsible for the clearance of TNF.

The present study adds further evidence to the role of the inflammatory response in ischemia-reperfusion associated injury. It is suggested that there is an interaction between tissue injury and inflammation that may aggravate both local ischemic damage and systemic effects of the inflammatory response. TNF seems to play a crucial role in this phenomenon. Further analysis of the true nature of the interaction whether mediated by TNF or other cytokines may rationalize treatment of ischemia-reperfusion associated injury either or not complicated by concomitant inflammatory disease. 


\section{REFERENCES}

Allaf ME, Chapelle JP, Allaf DE, Adam A, Faymonville ME, Laurent P, Heusghem C (1986) Differentiating muscle damage from myocardial injury by means of the serum creatine kinase $(\mathrm{CK})$ isoenzyme $\mathrm{MB}$ mass measurement/total $\mathrm{CK}$ activity ratio. Clin Chem 32:291-295.

Amador E, Dorfman LE, Wacker WEC (1965) Urinary alkaline phosphatase and LDH activities in the differential diagnosis of renal disease. Ann Int Med 62:30-40.

Arnold PE, Van Putten VJ, Lumlertgul D, Burke TJ, Schrier R (1986) Adenine nucleotide metabolism and mitochondrial $\mathrm{Ca}^{2+}$ transport following renal ischemia. Am J Physiol 250:F357-F363.

Atkinson DE (1968) The energy charge of the adenylate pool as a regulatory parameter. Interaction with feedback modifiers. Biochemistry 7:4030-4034.

Balint P, Laszlo K (1985) Effect of imidazole and indomethacin on hemodynamics of the obstructed canine kidney. Kidney Int 27:892-897

Basci A, Wallin D, Shah SV (1987) Effect of stimulated neutrophils on cyclic nucleotide content in isolated rat glomeruli. Am J Physiol 252:F429-F436

Beck TA (1979) Machine versus cold storage preservation and TAN versus the energy charge as a predictor of graft function posttransplantation. Transplant Proc 11:459-464.

Beeuwkes R (1980) III The vascular organization of the kidney. Ann Rev Physiol 42: 531542.

Beezhold DH, Best GK, Bonventre PF, Thompson M (1987) Synergistic induction of interleukin-1 by endotoxin and toxic shock syndrom toxin-1 using rat macrophages. Infect Immun 55:2865-2869

Belzer FO, Hoffmann RM, Rice MJ, Southard JH (1985) Combination perfusion-cold storage for optimum cadaver kidney function and utilization. Transplantation 39:118121.

Belzer FO, Sollinger HW, Glass NR, Miller DT, Hoffmann RH, Southard JH (1983) Beneficial effects of adenosine and phosphate in kidney preservation. Transplantation 36:633-635.

Bendtzen K, Mandrup-Poulsen T, Nerup J, Nielsen JH, Dinarello CA and Svensen M (1986) Cytotoxicity of human p17 Interleukin-1 for pancreatic islets of Langerhans. Science 232:1545

Benjamin JF, Sell KW (1972) Effects of temperature on kidneys preserved by hypothermic perfusion. Transplantation 14:501-510.

Bergstrom J, Collste H, Groth H, Hultman E, Melin B (1979) Water, electrolyte and metabolite content in cortical tissue from dog kidneys preserved by hypothermia. Proc Eur Dialysis Transplant Ass 8:313.

Beutler B, Cerami A (1987) Cachectin: more than a tumor necrosis factor. N Eng J Med 316:379-385

Bevilaqua MP, Pober JS, Majeau GR, Gotran RS, Gimbrone MA Jr. (1984). Interleukin 1 (II-1) induces biosynthesis and cell surface expression of procoagulant activity in human vascular endothelial cells. J. Exp. Med. 160:618.

Bhardway N, Nash TW and Horwitz MA (1986) Interferon-activated human monocytes inhibit the intracellular multiplication of legionella pneumophila. J. Immunol. 137:2662. Biliau A (1988) Gamma-interferon: the match that lights the fire? Immunology Today 
Billah MM, Finean JB, Coleman R, Michell RH (1977) Permeability characteristics of erythrocyte ghosts prepared inder isoionic conditions by a glycol-induced osmotic lysis. Biochim Biophys Acta 465:515-526.

Bore PJ, Papatheophanis I, Sells RA (1979) Adenosine triphosphate regeneration and function in the rat kidney following warm ischaemia. Transplantation 27:235-237.

Bourbouze R, Gluckman JC, Frantz P, Paraire M, Baumann FC, Luciani J, Percheron, Legrain $M$ (1985) Early monitoring of human renal transplantations by $\mathrm{N}$-acetyl-betaD-glucosaminidase isoenzyme activities in urines. Clin Chim Acta 149:185-195.

Braunwald E, Kloner RA (1982) The stunned myocardium: prolonged postischemic ventricular dysfunction. Circulation 66:1146-114.

Breslow MJ, Miller CF, Parker SD, Walman AT, Traystman RJ (1987) Effect of vasopressors on organ blood flow during endotoxin shock in pigs. Am J Physiol 252:H291H300.

Buhl MR (1979) The predictive value of the 5'-adenine nucelotide depletion and replenishment in ischaemic rabbit kidney tissue. Int Urol Nephrol 11:325-329.

Buhl MR, Kemp E, Kemp G (1977) Inosine in preservation of rabbit kidneys for transplantation. Transplant Proc 9:1603-1606.

Buhl MR, Kemp G, Kemp E (1976) Hypoxanthine excretion during preservation of rabbit kidneys for transplantation. Transplantation 21:460-467.

Buhl MR, Jensen MH (1979) The role of 5'nucleotidase in purine depletion of ischaemic renal tissue. In Pegg DE, Jacobsen IA. eds. Organ Preservation II Edinburgh;Churchill Livingstone

Busch EW, Von Borcke IM, Martinez B (1969) Abbauwege und Abbaumuster der Purinnucleotide in Herz- Leber- und Nierengewebe von Kaninchen nach Kreislaufstillstand. Biochim Biophys Acta 166:547-556.

Calman KC (1974) The prediction of organ viability: An hypothesis. Cryobiology 1:11-24. Chapman AG, Atkinson DE (1973) Stabilization of adenylate energy charge by the adenylate deaminase reaction. J Biol Chem 248:8309-8312.

Charlat ML, O’Niell P, Egan JM, Abernethy DR, Michael LH, Myers ML, Roberts $\mathbb{R}$, Bolli R (1987) Evidence for a pathogenitic role of xanthine oxidase in the stunned myocardium. Am J Physiol 252:H566-H577.

Chatterjee SN (1977) Pharmacologic agents of potential value in protecting kidneys from ischemic damage. Transplant Proc 9:1579-1582.

Chaudry IH (1983) Cellular mechanisms in shock and ischemia and their correction. Am J Physiol 245:R117-R134.

Cheung JY, Bonventr JV, Malis CD, Leaf A (1986) Calcium and ischemic injury. N Engl J Med 314:1670-1676.

Chia HL (1986) Particulate radiopharmaceuticals in nuclear medicine. In: Cox PH (ed) Radiopharmacology and radiopharmacy yearbook II. Grune and Stratton.

Chopra J, Joist JH, Webster RO (1987) Loss of chromium, lactate dehydrogenase, and Indium as indicators of endothelial cell injury. Lab Invest 57:579-584.

Coffman TM, Sanfilippo FP, Brazy PC, Yarger WE, Klotman PE (1986) Bilateral native nephrectomy improves renal isograft function in rats. Kidney int 30:20-26.

Cole BR, Hays AE, Boylan JG, Burch HB, Lowry OH (1982) Distribution of enzymes of adenylate and guanylate nucleotide metabolism in rat nephron. Am J Physiol 243:F349. 
F355.

Collins GM, Green RD, Halasz NA (1979) In witro regeneration of adenine nucleotide by ischemically injured kidney. World J Surg 3:367-373.

Cooper K, Stromski M, Thulin G, Gaudio K, Shulman R, Siegel N (1987) Postischemic renal ATP recovery: correlations with functional impairment. Kidney Int 31: (Abstract). Chopra J, Joist JH, Webster RO (1987) Loss of ${ }^{51}$ Chromium, lactate dehydrogenase, and ${ }^{111}$ Indium as indicators of endothelial cell injury. Lab Invest 57:578-584.

Cosimi AB, Colvin RB, Burton RC, Rubin RH, Goldstein G, Kung PC, Hansen WP, Delmonico FL, Russell PS (1981) Use of monoclonal antibodies to T-cell subsets for immunologic monitoring and treatment in recipients to renal allografts. N. Engl. J. Med. 305-308.

Cotran RS (1987) New roles for the endothelium in inflammation and immunity. Am J Pathol 129:407-413.

Cunnaro JA, Schultz SE, Johnson WA, Weiner MW (1982) Effects of ischemia on metabolite concentrations in dog renal cortex. Renal Phsyiol 5:143-155.

Das DK, Engelman RM, Rousou JA, Breyer RH, Otani H, Lemeshow S. (1986) Role of membrane phospholipids in myocardial injury induced by ischemia and reperfusion. Am J Physiol 251:H71-H79.

Dinarello CA, Clowes Jr. GHA, Gordon AH, Saravis CA and Wolff SM (1984) Cleavage of human interleukin 1: Isolation of a peptide fragment from plasma of febrile humans and activated monocytes. J. Immunol. 133:1332.

Dinarello CA, (1985) Interleukin-I. Rev. Infect. Dis. 6:51.

Donohoe JF, Venkatachalam MA, Bernard DB, Levinsky NG (1978) Tubular leakage and obstruction after renal ischemia:structural-functional correlations. Kidney Int 13:208222.

Doveren RFC, Buurman WA, Van der Linden CJ, Strijbosch LWG, Spronken EEM, Kootstra G (1986) Analysis of cytotoxic $T$ lymphocyte response in rejecting allografted canine kidneys. Transplantation 41:33.

Dubach UC, Schmidt U (1970) Enzymology of human kidney. Enzym biol clin 11:32-51. Duvall E, Wyllie AH (1986) Death and the cell. Immunol today 7:115-119.

Eliska O, Eliskova M, Mirejovski P (1986) Lymph vessels of the transplanted kidney. Nephron 44:136-141.

Elkins KL, Stashak PW, Baker PJ (1987) Mechanisms of specific immunological unresponsiveness to bacterial lipopolysaccharides Infect Immun 55:3093-3102.

Engler RL, Dahlgren MD, Peterson MA, Dobbs A, Schmid-Schonbein GW (1986) Accumulation of polymorphonuclear leukocytes during 3-h experimental myocardial ischemia. Am J Physiol 251:H93-H100.

Farber NE, Pieper GM, Thomas JP, Gross GJ (1988) Beneficial effects of iloprost in the stunned canine myocardium. Circ Res 62:204-215.

Feinberg H. (1979) Energetics and mitochondria. Pegg DE, Jacobson IA (eds). Organ preservation II Edinburgh;Churchill Livingstone..

Fernando AR, Griffiths JR, O'Donoghue EPN, Ward JP, Armstrong DMG, Hendry WF, Perret D, Wickham JEA. (1976) Enhanced preservation of the ischaemic kidney with inosine. Lancet 13:555-557.

Finn WF (1980) Enhanced recovery from post ischemic acute renal failure. Circ Res 46$440-448$ 
Fischer JH, Czerniak A, Hauer U, Isselhard W (1978) A new simple method for optimal storage of ischemically damaged kidneys. Transplantation 25:43-49.

Fischer JH, Armbruster D, Grebe W, Czerniak A, Isselhard W (1980) Effects of differences in substrate supply on the energy metabolism of hypothermically perfused canine kidneys. Cryobiology 17:135-147.

Fischer JH, Kulus D, Hansen-Schmidt I, Isselhard W (1981) Adenine nucleotide levels of canine kidneys during hypothermic aerobic or anaerobic storage in Collins' solution. Eur Surg res 13:178.

Fischer JH, Knupfer P, Beyer M (1985) Flush solution 2, a new concept for one-to-threeday hypothermic renal storage preservation. Transplantation 39:122-126.

Foreman J, Wusteman MC, Pegg DE: in Pegg DE, Jacobsen IA, Halasz NA (eds) (1982) Organ Preservation, MTP Press Limited, Lancaster ch 24.

Freeman DM, Chan L, Yahaya H, Holloway P, Ross BD (1986) Magnetic resonance spectroscopy for the detemination of renal meatbolic rate in vivo. Kidney Int 30:35-42.

Fried TA, Hishida A, Barnes JL, Stein JH (1984) Ischemic acute renal failure in the rat: protective effect of uninephrectomy. Am J Physiol 247:F568-F574

Fuhrman FA, Field J (1943) The reversibility of the inhibition of rat brain and kidneys metabolism by cold. Am J Physiol 139:193-196.

Gaber AO, Yang HC, Haag FC, et al (1987) Internediate normothermic hemoperfusion doubles safe cold preservation of rat kidneys. Transplant Proc 19:1369.

Garvin PJ, Castanedo M, Niehoff M, Jellinek M, Codd JE (1985) An experimental evaluation of nucleotide enhancement techniques for kdney transplantation. J Surg Res 38:2938.

Garvin PJ, Okiye S, Carney K, Jellinek M, Codd JE (1982) The effect of ATP-MgCL 2 and Dipyridamole pretreatment in renal preservation. J Surg Res 32:70-74.

Gaudio KM, Stromski M, Thulin G, Ardito T, Kasgarian M, Siegel NJ (1986) Postischemic hemodynamics and recovery of renal adenosine triphosphatase. Am J Physiol 251:F603F609.

Gerlach E, Marko P, Zimmer HG, Pechan I, Trendelenburg Ch (1970) Different response of adenine nucleotide synthesis de novo in kidney and brain during aerobic recovery from anoxia and ischemia. Experientia 27:876-878.

Gerlach E, Deuticke B, Dreisbach RH (1963) Zum Verhalten won Nucleotiden und ihren dephosphorylierten Abbauprodukten in der Niere bei Ischaemie und kurzzeitiger postischaemischer Wiederdurchblutung. Pflugers Arch 278:296-315.

Gowen M, Mundy GR (1986) Actions of recombinant interleukin 1, interleukin 2, and interferon- on bone resorption in vitro. J. Immmunol. 136:2478.

Good RA, Thomas L (1952) Studies on the generalized Shwartzman reaction II. J exp Med 95:625-641.

Green CJ, Healing G, Lunec J, Fuller BJ, Simpkin S (1986) Evidence of free-radical-induced damage in rabbit kidneys after simple hypothermic preservation and autotransplantation . Transplantation 41:161-165.

Gregg CM, Cos LR, Saraf P, Fridd CW (1986) Recovery of glomerular and tubular function of autotransplantated dog kidneys preserved by hypothermic storage or machine preservation. Transplantation 42:453-457.

Harmsen E, De Tombe PP, De Jong JW (1982) Simultaneous determination of myocardial adenine nucleotides and creatine phosphate by high-performance liquid chromato- 
graphy. I Chromatography 230:131-136.

Hawkins HE, Clark P, Lippert EC, Karow AM (1985) Functional preservation of the mamalian kidney VI. J Surg Res 38:281-288.

Henson PM, Johnston RB (1987) Tissue injury and inflammation. J Clin Invest 79:669674.

Hermens WTH, Willems GM, Visser MP (1982) Quantification of circulating proteins. Martinus Nijhoff Publishers 1982; The Hague: The Netherlands.

Herminghuysen D, Welbourne CJ, Welbourne TC (1985) Renal sodium reabsorption, oxygen consumption, and gamma-glutamyltransferase excretion in the postischemic rat kidney. Am J Physiol 248:F804-F809.

Hernandez LA, Grisham MB, Twohig B, Arfors KE, Harlan JM, Granger DN (1987) Role of neutrophils in ischemia-reperfusion-induced microvascular injury. Am J Physiol 253:H699-H703

Hochachka PW (1986) Defense strategies against hypoxia and hypothermia. Science 231:234-241.

Horpacsy G, Schnells G (1980) Metabolism of adenine nucleotides in the kidney during hemorrhagic hypotension after recovery. J Surg Res 29:11-17.

Hostetter TH, Olson JL, Rennke HG, Venkatachalam MA, Brenner BM (1981) Hyperfiltration in remnant nephrons: a potentially adverse response to renal ablation. Am J Physiol 241:F85-F93.

Isselhard W, Berger M, Denecke H, Witte J, Fischer JH, Molzberger (1972) Metabolism of canine kidneys in anaerobic ischemia and in aerobic ischemia by persufflation with gaseous oxygen. Pglugers Arch 337:87-106.

Jacobsen LA, Chemnitz J, Kemp : Pegg DE, Jacobsen LA, Halasz NA (eds) (1982) Organ Preservation, MTP Press Limited, Lancaster ch 23.

Johnson RWG, Anderson M, Flear CTG, Murray SGH, Taylor RMR, Swinney J (1972) Evaluation of a new perfusion solution for kidney preservation. Transplantation 13:270275.

Johnson RWG, Anderson M, Morley AR, Taylor RMR, Swinney J (1972) Twenty-four hour preservation of kidneys injured by prolonged warm ischaemia. Transplantation 13:174-179.

Johnson RWG (1972) Brit I Surg 59: 765.

Jorgensen PL (1986) Structure, function and regulation of NA, K-ATPase in the kidney. Kidney Int 29:10-20.

Jung K, Pergande H, Schreiber G, Schröder K (1983) Stability of enzymes in urine at 37C. Clinica Chimica Acta 131: 185-191.

Kako K, Kato M, Matsuoka T, Mustapha (1988)Depression of membrane-bound Na-KATPase activiy induced by free radicals and by ischemia of kidneys. Am J Physiol 254:C330-C337.

Kallerhof M, Holscher M, Kehrer G, Klass G, Bretschneider H (1985) Effects of preservation conditions and temperature on tissue acidification in canine kidneys. Transplantation 39:485-489.

Kallerhof M, Blech M, Kehrer G, Kleinert H, Langheinrich M, Siekmann W, Helmchen U, Bretschneider HJ (1987) Short-term perfusion and "equilibration" of canine kidneys with protective solutions. Urol Res 15:5-12.

Keane WF, Gekker G, Schlievert PM, Peterson PK (1986) Enhancement of endo- 
toxin-induced isolated renal tubular cell injury by toxic shock syndrome toxin 1. Am J Pathol 122:169-176

Kelly CJ, Zurier RB, Krakauer KA, Blanchard N, Neilson EG (1987) Prostaglandin E1 inhibits effector $\mathrm{T}$ cell induction and tissue damage in experimental murine nephritis. $\mathrm{J}$ Clin Invest 79:782-789

Kelley J, Rozek MM, Suenram CA, Schwartz CJ (1988) Activation of human peripheral blood monocytes by lipoproteins. Am J Pathol 130:223-231.

Kerr WK, Kyle VN, Keresteci AG, Smythe CA (1960) Renal hypothermia. J Urol 81:236242.

Kesner L, Kang O, Kountz SL (1977) Stability of four key gluconeogenic enzymes during hypotheric storage. Transplant Proc 9:1573-1575.

Kettelhut IC, Fiers W, Goldberg AL (1987) The toxic effects of tumor necrosis factor in vivo and their prevention by cyclooxygenase inhibitors. Proc Natl Acad Sci 84:42734277.

Kim M, Akera T (1987) $\mathrm{O}_{2}$ free radicals: cause of ischemia-reperfusion injury to cardiac $\mathrm{Na}^{+}-\mathrm{K}^{+}$-ATPase. Am J Physiol 252:H252-H257.

Kobayashi T, Okamoto H, Yamada J, Setaka M, Kwan T (1984) Vesiculation of platelet plasma membranes . Biochim Biophys acta 778:210218.

Kootstra G, Ruers TJM, Vroemen JPAM (1986) The non-heart-beating donor: Contribution to the organ shortage. Transplant Proc 18:1410-1412.

Koyama I, Bulkley GB, Williams GM, Im MJ (1985) The role of oxygen free radicals in mediating the reperfusion injury of cold-preserved ischemic kidneys. Transplantation 40:590-595.

Kreisberg JI, Mills JW, Jarrel JA, Rabito CA, Leaf A (1980) Protection of cultured renal tubular epithelial cells from anoxic cell swelling and cell death. Proc Natl Acad Sci 77:5445-5447.

Kruuv J, Glofcheski D, Cheng KH, et al (1983) Factors influencing survival and growth of mammalian cells exposed to hypothermia. I. Effects of temperature and membrane lipid perturbers. J. Cell Physiol 115: 179.

Lambert R, Henry M, Howden B, Jablonski P, Rae D, Tavanlis G, Marshall V, Tange J (1986) Glomerular damage after kidney transplantation. Transplantation 42:125-130.

Lefer AM (1987) Interaction between myocardial depressant factor and vasoactive mediators with ischemia and shock. Am J Physiol 252:R193-R205

Lehmann V, Freudenberg MA, Galanos C (1987) Lethal toxicity of liposaccharide and tumor necrosis factor in normal and d-Galactosamine treated mice. J Exp Med 165:657663.

Liebau G, Klose HJ, Fischbach H, Pichlmayer H (1971) Simple tests for viability of the hypothermic pulsatile perfused dog kidney. Surgery 70:459-466.

Le Hir M, Angielski S, Dubach UC (1985) Properties of an ecto 5' nucleotidase of the renal brush border. Renal Physiol 8:321-327.

Lord RHH, Hancock WW, Colby AJ, Padberg W, Diamantstein JW, Kupiec-Weglinski JW, Tilney NL (1987). Effects of anti-I12 receptor monoclonal antibody and Cyclosporine on I12 receptor-positive cells infiltrating cardiac allografts in the rat. Transplant Proc. 19:354.

Loveland BE, Hogarth PM, Ceredig RH, Mckenzie IFC (1981) Cells mediating graft rejection in the mouse. J. Exp. Med. 153:1044. 
Lovett $\mathrm{DH}$, Resch $\mathrm{K}$, Gemsa $\mathrm{D}$ (1987) Interlekin I and the glomerular mesangium $\Pi$. Am J Pathol 129:543-551.

Lowry RP, Blais D, Marghesco D, Powell WS (1987) Immune effector mechanisms in organ allograft rejection VIII: Inflammatory mediators and cytotoxins in rejecting rat cardiac allografts. Transplant. proc. 19:424.

Lundstam S, Jonsson O, Petterson S, Schertsen T (1977) Glucose, Fatty acid and amino acid utilization during hypothermic perfusion of dog kidneys. Transplant Proc 9:15611563.

Maessen JG, Vork M, Kootstra G (1986a) Incomplete wash-out with Eurocollins in ischemic kidneys. Eur Surg Res 18 SI:35 (abstract).

Maessen JG, van der Vusse GJ, Vork M, Coumans WA, Kootstra G (1987a) To a valuable assessment of warm ischemia time in donor kidneys by analysis of purine metablolism. Transplant proc, 19:1365-1367.

Maessen JG, Hermens WTH, Vork M, Willems GM, Kootstra G (1987b) Appearance of enzymes in plasma or urine following renal injury. Nephrol Dial Transplant 2:17-21.

Maessen JG, van der Vusse GJ, Vork M, Kootstra G (1987c) Inability to maintain adenine nucleotide levels by cold storage in ischemically damaged and control kidneys. Transplant proc. 19:2114-4115.

Maessen JG, van der Vusse GJ, Vork M, Kootstra G (1987d) New approach to preservation of non-hearbeating donor kidneys: total body cooling. Tranplant proc 19:4159-4161.

Maessen JG, van der Vusse GJ, Vork M, Kootstra G (1988a) Determination of warm ischemia time at donornephrectomy. Transplantation 45:147-152

Maessen JG, van der Vusse GJ, Vork M, Kootstra G (1988b) Assessment of nucleotides, nucleosides, and oxypurines in human kidneys with the use of reversed-phase high performance liquid chromatography. Clin Chem 34:1087-1090.

Maessen JG, van der Vusse GJ, Vork M, Kootstra G (1988c) Assessment of purine metabolites in human donor kidneys. Transplant proc in press.

Maessen JG, van der Vusse GJ, Vork M, Kootstra G (1988d) Potentiation of ischemic injury during hypothermic storage in donor kidneys: role of the energy metabolism. Transplant proc in press.

Maessen JG, Greve J-WM, Buurman WA (1988e) Role for TNF in kidney transplant injury. Transplant proc in press.

Maessen JG, Buurman WA (1988f) Direct cytotoxic effect of cytokines: a possible mechanism of allograft destruction. Transplant proc in press.

Maessen JG, Ruissen K, Kootstra G (1988g) Assessment of postischemic changes in intrarenal blood flow distribution. Transplant proc in press.

Maessen JG, wan der Vusse GJ, Vork M, Kootstra G (1988h) Improved preservation of ischemic donor kidneys. Transplant proc in press.

Maessen JG, Hermens WTH, Vork M, Kootstra G (1988i) The assessment of actual cellular damage in renal failure. Transplant proc in press.

Maessen JG, Hermens WTH, Vork M, Kootstra G, Estimation of renal ischemic damage from quantitated enzyme leakage in plasma. (submitted)

Maessen JG, Hermens WTH, Vork M, Kootstra G (1988k) Loss of enzymes from viable kidney tissue. (submitted)

Maessen JG, van der Vusse GJ, Vork M, Kootstra G (19881) The stunned kidney: postischemic renal failure and adenine nucleotide homeostasis. (submitted) 
Maessen JG, van der Vusse GJ, Vork M, Kootstra G (1988m) The beneficial effect of intermediate normothermic perfusion during cold storage of ischemically injured kidneys. Transplantation, in press.

Maessen JG, Buurman WA (1988n) Interleukin 1 and interferon gamma mediated cytotoxicity of kidney tubular cells. (submitted)

Maessen JG, Greve J-WM, Buurman WA (19880) Potentiation of ischemic acute renal failure by LPS is mediated by TNF. (submitted)

Maessen JG, Hermens WTH, Vork M, Kootstra G (1988p) Quantitation of renal enzyme loss and differentiation from skeletal muscle enzyme loss following renal transplantation and surgery. Kidney Int in press (abstract)

Mandel LJ (1986) Primary active sodium transport, oxygen consumption, and ATP: Coupling and regulation. Kidney Int 29:3-9.

Marshall V, Ross B, Bishop M, Morris P (1978) Evaluation of renal preservation using the isolated perfused rat kidney. Transplantation 26:315-318.

Marshall V, Ross B, Smith M, Barlett S, Freeman D (1985) Organ and tissue preservation for transplantation: Monitoring by ${ }^{31} \mathrm{p}$ nuclear magnetic resonance. Transplant Proc 17:1693.

Marubayashi S, Takenaka M, Dohi K, Ezaki H, Kawasaki T (1980) Adenine nucleotide metabolism during hepatic ischemia and subsequent blood reflow periods and its relation to organ viability. Transplantation 30:294-296.

Mason J, Welsch J, Torhorst J (1987) The contribution of vascular obstruction to the functional defect that follows renal ischemia. Kidney Int 31:65-71.

Mattenheimer H (1968) Enzymology of kidney tissue. Enzymes in urine and kidney. Current problem in clin. biochem. 2, Hans Huber Verlag, Bern, 119-145.

Mattenheimer H (1977) Enzymes in renal diseases. Ann Clin Lab Sci 7:422-432.

Mauel J, Buchmuller-Rouiller Y (1987) Effect of lipopolysaccharide on intracellular killing of Leishmania enriettii and correlation with macrophage oxidative metabolism. Eur J Immunoll 17:203-208.

McCord JM (1985) Oxygen-derived free radicals in postischemic tissue injury. N Engl J Med 312:159-163.

Mayfield KB, Ametani M, Southard JH, Belzer FO (1987) Mechanisms of action of ex vivo blood rescue in six-day preserved kidneys. Transplant Proc 19:1367.

Mees N, Southard JH, Belzer FO (1982) Inhibition of ischernic induced cellular swelling in kidney cortex tissue by lactobionate anions. J Trauma 22:118-120.

Milton AD, Fabre JW (1985). Massive induction of donor-type class I and class II major histocompatibility complex antigens in rejecting cardiac allografts in the rat. J. Exp. Med. 161:98.

Miossec, P. Ziff M (1986) Immune interferon enhances the production of interleukin I by humna endothelial cells stimulated with lipopolysaccharide. J. Immunol. 137:2848.

Morrison DC, Ulevitch RJ (1978) The effects of bacterial endotoxins on host mediation systems. Am J Pathol 93:527617.

Movat HZ, Burrowes CE, Cybulsky MI, Dinarello CA (1987) Acute inflammation and a Shwartzman-like reaction induced by interleukin-1 and tumor necrosis factor. Am J Pathol 129:463-476.

Murray AW (1971) The Biological significance of purine salvage. Ann Rev Biochem 40: 811-831. 
Old LJ (1987) Another chapter in the long history of endotoxin. Nature 330:602-603. Onozaki K, Matsushima K, Aggarwal BB, Oppenheim JJ (1985) Human Interleukin 1 is a cytocidal factor for several tumor cell lines. J. Immunol. 135:3962.

Oostenbroek RJ, Willems GM, Boumans MLL, Soeters PB, Hermens WTH (1985) Liver damage as a potential source of error in the estimation of myocardial infarct size from plasma creatine kinase activity. Cardiovasc Res 19:113-119.

Osswald H, Schmitz HJ, Kemper R (1977) Tissue content of adenosine, inosine and hypoxanthine in the rat kidney after ischemia and postischemic recirculation. Pfluegers Arch 371:45

Paddock JK, Lada W, Lowenstein LM (1981) Regeneration of the renal brush border after renal ischemia in rats. Am J Physiol 241:F28-F33.

Pearson JD, Gordon JL (1979) Vascular endothelial and smooth muscle cells in culture selectively release adenine nucleotides. Nature 281:384-386.

Pegg DE (1978) An approach to hypothermic renal preservation. Cryobiology 15:1-17.

Pegg DE (1985) Principles of tissue preservation. In: Morris PJ, Tilney NL (eds) Progress in Transplantation . Churchill Livingstone, Edinburgh:69-106.

Pegg DE, Wusteman MC, Foreman J (1981) Metabolism of normal and ischemically injured rabbit kidneys during perfusion for 48 hours at $10 \mathrm{C}$. Transplantation 32:437-443.

Pegg DE, Foreman J, Rolles K (1984) Metabolism during preservation and viability of ischemically injured canine kidneys. Transplantation 38:78-81.

Persson NH, Erlansson M, Bergqvist D, Takolander R, Svensjo E (1987) Terbutaline and budesonide as inhibitors of postischaemic permeability increase. Acta Physiol Scand 129:517-524.

Piquet PF, Grau GE, Allet B, Vassalli P (1987). Tumor necrosis factor/cachectin is an effector of skin and gut lesions of the acute phase of graft-vs.-host disease. J. Exp. Med. 166:1280.

Plummer DT, Ngabe EO, Wright PJ, Leathwood PD, Blake ME (1979) The sensitivity of urinary enzyme measurements for detecting renal injury. Diagnostic significance of enzymes and proteins in urine. Dubach UC, Schmidt U (eds), Hans Huber Verlag, Bern, 71-87. Price RG (1982) Urinary enzymes, nephrotoxicity and renal disease. Toxicology 23:99-134.

Prout GR, Macalalag EV, Hume DM (1964) Serum and urinary lactic dehydrogenase in patients with renal homotransplants. Surgery 56:283-295.

Quebbemann AJ, Cumming JD, Shideman JR, Toledo-Pereyra L. (1975) Synthesis of uric acid in isolated normothermic perfused mongrel and Dalmatian dog kidneys. Am J Physiol 228:959-963.

Raab WP (1972) Diagnostic value of urinary enzyme determinations. Clin Chem 18:5-25. Raij L, Keane WF, Michael AF (1977) Unilateral Shwartzman reaction: cortical necrosis in one kidney following in vivo perfusion with endotoxin. Kidney Int 12:91-95.

Reed EB (1976) Coordination of adenylate energy charge and phosphorylation state during ischemia and under physiological conditions in rat liver and kidney. Life Sci 19:13071322.

Reimer KA, Hill ML, Jennings RB (1981) Prolonged depletion of ATP and of the adenine nucleotide pool due to delayed resynthesis of adenine nucleotides following reversible myocardial ischemic injury in dogs. J Mol Cell Cardiol 13:229-239.

Rice MJ, Southard JH, Hoffmann RM, Belzer FO (1985) Effects of hypothermic kidney 
preservation on the isolated perfused kidney: a comparison of reperfusion methods. Cryobiology 22:161-167.

Rijkmans BG, Van Der Wijk J, Donker AJM, Slooff MJH, Kootstra G. (1982) Functional studies in 6 days successful preserved canine kidneys. J Urol 127:163-166.

Rijkmans BG, Buurman WA, Kootstra G (1984) Six-day canine kidney preservation. transplantation 37:130-134.

Ringoir S (1968) Correlations between kidney, serum and urinary enzyme activity. Enzymes in urine and kidney. Current problem in clim. biochem. 2, Hans Huber Verlag Bern:177-199.

Roberts R, Henry PD, Sobel BE (1975) An improved basis for enzymatic estimation of infart size. Circulation 52:743-754.

Rolles K, Foreman J, Pegg DE (1984) Preservation of ischemically injured canine kidneys by retrograde oxygen persufflation. Transplantation 38:102-106.

Ross B, Freeman D, Chan L (1986) Contributions of nuclear magnetic resonance to renal biochemistry. Kidney Int 29:131-141.

Ross H, Escott ML (1979) Gaseous oxygen perfusion of the renal vessels as an adjunct in kidney preservation. Transplantation 28:362-364.

Ruers TJM, Buurman WA, Van Boxtel CJ, Van der Linden CJ, Kootstra G (1987) Immunohistological observations in rat kidney allografts after local steroid administration. J. Exp. Med. 166:1205.

Ruggiero V, Baglioni C (1987) Synergistic anti-proliferative activity of interleukin 1 and tumor necrosis factor. J Immunol 138:661-663.

Schloerb PR, Waldorf RD, Welsh JS 1957 The protective effect of kidney hypothermia on total renal ischemia. Urology 8:633-635.

Schlondorff D, Neuwirth R (1986) Platelet-activating factor and the kidney. Am J Physiol 251:F1-F11.

Schmidt E, Schmidt F (1976) Enzyme diagnosis in diseases of the liver abd biliary system. Clin Enzymol, Karger, Hannover, 239-293.

Schmidt-Mende H (1966) Der energiestoffwechsel der kaltekonservierten niere. Langenbecks Arch klin Chir 316:894-898.

Segaul RM, Lytton B, Schiff M (1972) Functional aspects of compensatory growth after ischemic injury. Invest Urol 10:235-238.

Sells RA, Bore PJ. Mclaughlin GA, Johnson JN, Tyrrell I (1977) A predictive test of renal viability. Transplant Proc 9:1557-1560.

Shapiro JI, Cheung C, Itabashi A, Chan L, Schrier RW (1985) The effect of verapamil on renal function after warm and cold ischemia in the isolated perfused rat kidney. Transplantation 40:596-600.

Sheehan HL, Davis JC (1959) Renal ischaemia with failed reflow. J. Path Bact 78:105.

Shell WE, Kjekshus JK, Sobel BE (1971) Quantitative Assessment of the extent of myocardiall infarction in the conscious dog by means of analysis of serial changes in serum creatine phosphokinase activity. J Clin Invest 50:2614-2625.

Shepherd RE, Lang CH, Mcdonough KH (1987) Myocardial adrenergic responsiviness after lethal and nonlethal doses of endotoxin. Am J Physiol 252:H410-H416.

Shiki Y, Meyrick O, Brigham KL, Burr IM (1987) Endotoxin increases superoxide dismutase in cultered bovine pulmonary endothelial cells. Am J Physiol 252:C436C440. 
Sinsteden TD, O'Niel TJ, Hill S, Lifschitz MD, Stein JH (1986) The role of high-energy phosphate in norepinephrine-induced acute renal failure in the dog. Circ Res 59:93-104. Solez K (1984) The pathology and pathogenesis of human acute tubular necrosis. Acute renal failure Solez $k$ (ed) Marcel Dekker $\mathrm{NY}$.

Simoons ML, Serruys PW, Van den Brand M, et al (1986) Early thrombosis in acute myodarcial infaction: Limitation of infarct size and improved survival. J Am Coll cardiol 7: 7117-28.

Southard JH, Lutz MF, Ametani MS, Belzer FO (1984) Stimulation of ATP synthesis in hypothermically perfused dog kidneys by adenosine and PO4. Cryobiology 21:13-19.

Southard JH, van der Laan NCHR, Lutz M, Pavlock GS, Belzer JP, Belzer FO (1983) Comparison of the effect of temperature on kidney cortex mitochondria from rabbit, dog, pig and human: Arrhenius plots of ADP-stimulated respiration. Cryobiology 20:395-400.

Southard JH, Senzig KA, Hoffmann RM, Belzer FO (1977) Energy metabolism in kidneys stored by simple hypothermia. Transplant Proc 9:1535-1539.

Southard JH, Rice MJ, Belzer FO (1985) Preservation of renal function by adenosinestimulated ATP synthesis in hypothermically perfused dog kidneys. Cryobiology 22:237242.

Southard JH, Senzig KA, Belzer FO (1980) Effects of hypothermia on canine kidney mitochondria. Cryobiology 17:148-153.

Southard JH, Kuniyoshi M, Lutz MF, Ametani M, Belzer FO (1984) Comparison of the effect of 3 and 5-day hypothermic perfusion of dog kidneys on metabolism of tissue slices. Cryobiology 21:285-295.

States B, Holtzapple P, Rosenhagen M, Segal S (1972) Effect of hypothermic storage of kidney slices on membrane ATPases and electrolyte transport. Kidney Int. 2:17-21.

Sumpio BE, Chaudry IH, Clemens MG, Baue AE (1984) Accelerated functional recovery of isolated rat kidney with ATP-MgC12 after warm ischemia. Am J Physiol 247:R1047R1053.

Sumpio BE, Hull MJ, Baue AE, Chaudry IH (1987) Comparison of effects of ATP-MgC12 and adenosine-MgC12 on renal function following ischemia. Am J Physiol 252:R388R393.

Szabo G, Magyar Z (1980) Biochemical changes in the body fluids after ischaemic tissue injury. Lymphology 13:74-77.

Szabo G (1976) Movement of proteins into blood capillaries. Ergebnisse der Angiologie. Basic Lymphology, Földi M (ed), Stuttgart, FK Schattenhauer Verlag 31-30.

Szabo G, Magyar Z (1978) The relationship between tissue fluid and lymphII. Enzymes in tissue fluid and lymph. Lymphology 11:101-105.

Szabo G, Magyar Z (1974) Intracellular enzymes in serum, lymph and urine after renal ischaemia. Lymphology 7:13-22.

Takenaka M, Tatsukawa Y, Yamane S, Tanaka I, Dohi K, Ezaki H, Tracey KJ, Fong Y, Hesse DG, Manogue KR, Lee AT, Kuo GC Lowry SF, Cerami A (1987) Anti-cachectin/TNF monoclonal antibodies prevent septic shock during lethal bacteraemia. $\mathrm{Na}$ ture 330:662-664.

Takenaka M, Tatsukawa Y, Yamana S, et al (1980) An experimental model to test the viability of ischemic kidney. Transplantation 30:311-312.

Tessitore N, Sakhrani LM, Massry SG (1986) Quantitative requirements for ATP for ac- 
tive transport in isolated cells. Am J. Physiol 251:C120-C127.

Thorens B, Mermod J-J, Vassalli" P (1987) Phagocytosis and inflammatory stimuli induce GM-CSF mRNA in macrophages through posttranscriptional regulation. Cell 48:671679.

Tatsukawa Y, Dohi Y, Yamada K, Kawasaki T (1979) The role of coenzyme Q10 for preservation of the rat kidney: a model experiment for kidney transplantation. Life Sci 24:1309-1314.

Urbaitis BK (1984) Effect of ischemia and hypertonic saline loading on renal adenine nucleotides. Renal physiol 7:22-31.

Van der Laarse A, Hollaar L, van der Valk LJM (1979) Release of alpha hydroxybutyrate from neonatal rat heart cell cultures exposed to anoxia and reoxygenation: comparison with impairment of structure and function of damaged cardiac cells. Cardiovasc Res 13:345-353.

Vanderlinde RE (1981) Urinary enzyme measurements in the diagnosis of renal disorders. An Clin Lab Sci 11:189-201.

Van der Vusse GJ, Coumans WA, Van der Veen E, Drake AJ, Flameng W, Suy R (1984) ATP, creatine phosphate and glycogen content in human myocardial biopsies: Markers for the efficacy of cardioprotection during aorta-coroby bypass surgery. Vasc Surg 18:127.

Van der Wijk J, Slooff MJH, Rijkmans BG, Kootstra G (1980) Successful 96-and 144-hour experimental kidney preservation: A combination of standard machine preservation and newly developed normothermic ex vivo perfusion. Cryobiology 17:473.

Van der Wijk J. Rijkmans BG, Kootstra G (1982) Six day kidney preservation in a canine model. In fluence of a 1 to $4 \mathrm{hr}$ ex-vivo perfusion interval. Transplantation 35:408-411.

Vatner SF, Patrick TA, Knight DR, Manders WT, Fallon JT (1988) Effects of calcium channel blocker on responses of blood flow, function, arrhythmias, and extent of infarction following reperfusion in conscious baboons. Circ Res 62:105-115.

Vedder NB, Winn RK, Rice CL, Chi EY, Arfors KE, Harlan JM (1988) A monoclonal antibody to the adherence-promoting leukocyte glycoprotein, CD18, reduces organ injury and improves survival from hemorrhagic shock and resuscitation in rabbits. $J$ Clin Invest 81:939-944.

Venkatachalam MA, Patel YJ, Kreisberg JI, Weinberg JM (1988) Energy tresholds that determine membrane integrity and injury in a renal epithelial cell line (LLC-PK1). J Clin Invest 81:745-758.

Visser MP, Krill MTA, Muijtjens AMM, Willems GM, Hermens WTH (1981) Distribution of enzymes in dog heart and liver; Significance for assessment of tissue damage from data on plasma enzyme activities. Clin Chem 27:1845-1850.

Voss R, Ruile K, Vogell W, Kunz W (1970) Untersuchungen zur ischaemietoleranzzeit der Nieren. Klinische Wochenschrift 48:1089-1096.

Warnick CT, Lazarus HM (1977) Adenine nucleotides during organ storage. Transplant Proc 9:1575-1577.

Welbourne TC, Phifer T, Thomas M, Dass PD (1983) Gamma-glutamyltransferase release by the postischemic kidney: Multiple forms and cellular $\mathrm{pH}$. Life Sci 33:1141-1147.

Weidemann, Hems DA, Krebs HA (1969) Effects of adenine nucleotides on renal metabolism. Nephron 6:282-296.

Weinberg JM, Humes HD (1986) Increases of cell ATP produced by exogenous adenine 
nucleotides in isolated rabbit kidney tubules. Am J Physiol 250:F720-F733.

White $\mathrm{AG}_{x}$ Kumar MSA, Silva OSG, et al (1987) Levels of ATP and graft function in human cadaver kidneys with prolonged cold storage. Transplantation Proc 4168-4170.

Wichkam JEA, Hanley HG, Joekes AM (1967) Br J Urol 34:727.

Willems GM, Visser MP, Krill MTA, Hermens WTH (1982) Quantitative analysis of plasma enzyme levels based upon simultaneous determination of different enzymes. Cardiovase Res 16:120-131.

Willems GM. (1987) Thesis, Maastricht, The Netherlands.

Willems GM, Van Der Veen FH, Huysmans HA, Flameng W, De Meyere R, Van Der Laarse A, Van Der Vusse GJ, Hermens WTH (1985) Enzymatic assessment of myocardial necrosis after cardiac surgery: differentation from skeletal muscle damage, hemolysis, and liver injury. Am Heart J 109:1243-1252.

Willems G. Visser MP, Krill MTA, Hermens WT (1982) Quantitative analysis of plasma enzyme levels based upon simultaneous determination of different enzymes. Cardiovasc Res 16: 120-131.

Wilson PD, Schrier RW (1986) Nepron segment and calcium as determinants of anoxic cell death in renal cultures. Kidney Int 29:11721179.

Wynants J, Van Belle H (1985) Single-run high-performance liquid chromatography of nucleotides, nucleosides, and major purine bases and its application to different tissue extracts. Analytic Biochem 144:258-266.

Yamada K, Kawasaki T (1980) An experimental model to test the viability of ischemic kidney. Transplantation 30:311-312.

Young JD-E, Clark WR, Liu C, Cohn ZA (1987) A calcium- and perforin-independent pathway of killing mediated by murine cytolytic lymphocytes. J Exp Med 166:18941899.

Zager RA (1986) Escherichia coli endotoxin injections potentiate experimental ischemic renal injury. Am J Physiol 251:F988-F994.

Zager RA, Teubner EJ, Adler S (1987) Low molecular weight proteinuria exacerbates experimental ischemic renal injury. Lab Invest 56:180-188.

Zager RA, Venkatachalam MA (1983) Potentiation of ischemic renal injury by amino acid infusion. Kidney Int 24:620-625.

Zager RA, Altschuld R (1986) Body temperature: an important determinant of severity of ischemic renal injury. Am J Physiol 251:F87-F93.

Zavortink M, Thacher T, Rechsteiner MC (1979) Degradation of proteins microinjected into cultured mammalian cells. J. Cell. Physiol. 100-175. 


\section{SUMMARY}

This thesis presents the results of experimental studies on the evaluation of ischemic injury in kidneys for transplantation. During harvesting and transplantation of kidneys a number of situations arise in which ischemia may occur. Especially kidneys from donors with circulatory arrest (non-heartbeating donors) may have suffered from a considerable period of warm ischemia. Posttransplant viability of kidneys is negatively influenced by ischemia. The extent and effect of ischemic injury often remains obscure and only becomes evident some time after implantation. Mechanisms were studied that are likely to determine the effect of ischemia on renal viability in order to obtain parameters for monitoring ischemic injury. Three major points of interest were considered: the cellular energy metabolism, enzyme leakage from damaged cells and the involvement of inflammatory processes.

In part 1 data from the experimental studies are introduced and discussed in view of these points of interest and with respect to the results from previous studies by others on the same subject. Chapter 1,2, and 3 are dealing with the cellular energy metabolism, enzyme leakage from damaged cells, and inflammatory processes, respectively.

In part II the experimental studies are presented separately, in a similar fashion as they have been published or submitted for publication. Chapter 4 describes the development of a test to determine warm ischemia time in donor kidneys. In dog the concentrations of energy metabolites after various periods of warm ischemia were measured. Small changes in temperature affect the break down of adenine nucleotides. The ratio of the renal tissue content of adenine nucleotides and degradation products during ischemia correlates with the length of ischemia time. Tissue specimen for biochemical assessment of these metabolites can be harvested with a simple surgical wedge biopsy technique. Possible clinical application of this test is discussed.

Chapter 5 describes the effect of hypothermic storage on the loss of adenine nucleotides in canine kidneys during ischemia. The loss of these metabolites is highest in kidneys with high levels of adenine nucleotides before hypothermia is started. A nadir level of adenine nucleotide tissue content is reached after 72 hours of hypothermia irrespective of prior warm ischemic injury. Assessment of the adenine nucleotide content during hypothermia does not allow the estimation of prior warm ischemia time without additional information.

Chapter 6 describes the effectiveness of total body cooling as a method to initiate preservation in a study in rats. Body cooling from $37^{\circ} \mathrm{C}$ to $9.2^{\circ} \mathrm{C}$ within one hour, considerably reduces the loss of adenine nucleotides in kidneys following circulatory arrest. A simple and non-invasive method to initiate preservation, like total body cooling, may avoid technical and legal problems in harvesting non-heartbeating donor kidncys.

Chapter 7 describes the effect of warm ischemia with or without subsequent cold storage on the concentration of energy metabolites testestestesin renal tissue during reperfusion. The adenine nucleotide concentration increases within 1 hour of in situ perfusion dependent on prior warm ischemia time. Following warm ischemia and 48 hours cold storage no net increase is observed after 1 hour reperfusion. The combination of cold storage and warm ischemia prolongs the depletion of the adenine nucleotide pool. There is no evidence that locally accumulated degradation products are involved in the resynthesis of adenine nucleotides. The ability to resynthesize ATP is maintained. 
Chapter 8 describes a modified HPLC technique to determine tissue levels of 19 metabolic substances including adenine nucleotides, guanine nucleotides and a number of degradation products in renal tissue. Tissue specimen as small as $5 \mathrm{mg}$ of tissue wet weight are large enough to meet analytical demands. The intra and inter-assay coefficient of variance remains within acceptable limits. The concentration of nucleotides in cortical tissue is similar in human and canine kidneys.

Chapter 9 describes the relationship between early and late recovery of adenine nucleotides concentrations following ischemia and postischemic life sustaining function of kidneys. Kidneys after 90 minutes of ischemia are different from kidneys after 30 minutes of ischemia as appears from a depressed recovery of tissue adenine nucleotide concentration and lack of life sustaining function. Five days after ischemia, however, the concentration of adenine nucleotide in kidneys with 90 minutes of ischemia is similar as in kidneys with 30 minutes of ischemia. These kidneys recover to life sustaining function albeit only after five weeks. During this period processes other than the adenine nucleotide homeostasis determine the lack of life sustaining function.

Chapter 10 describes the adenine nucleotide homeostasis and viability of kidneys after warm ischemia and cold storage with or without an intermediate normothermic perfusion half-way the storage period. This treatment has a nucleotide sparing effect and improves posttransplant viability of kidneys. It is further described that flushing with Eurocollins induces a loss of adenine nucleotides which is larger than might be expected from cooling in ischemic conditions per se.

Chapter 11 describes the transport of extracellular cytosolic enzyme activity from renal tissue. Enzymes injected in parenchyma from canine kidneys, were rapidly and almost completely recovered in the blood compartment and not in the urinary compartment. A similar recovery in plasma is observed if the injections are performed in ischemically damaged tissue. Spontaneous release of enzyme activity following renal ischemia causes an increase in plasma enzyme activity and a negligible accumulation of enzyme activity in urine. Quantitative estimation of cytosolic enzyme leakage from ischemically damaged renal tissue should be based on changes in plasma enzyme activity.

Chapter 12 describes the relationship between the loss of enzyme activity from ischemically damaged renal tissue and changes in plasma enzyme activity. The cumulative amount of enzyme activity that enters the vascular compartment during a period of time was calculated according to a two-compartment model for circulating proteins. The total activity of AST that is recovered in plasma during 48 hours represents about $90 \%$ of the AST activity that is lost from ischemic renal tissue during this time. It is concluded that the depletion of renal tissue enzyme activity following ischemia can be estimated from changes in plasma AST activity. To allow proper calculation of the cumulative amount of enzyme activity in plasma, frequent blood sampling during the first 24 hours after ischemia is necessary. Differences in enzyme recovery in plasma following either permanent ischemia or transient ischemia with reperfusion suggest that reperfusion is associated with an additional increase in renal tissue enzyme loss.

Chapter 13 describes the relationship between renal tissue enzyme leakage and irreversible cell damage. Following 90 minutes of ischemia the renal tissue enzyme activity is reduced to about $50 \%$ during 48 hours. The renal tissue adenine nucleotide content, however, is near normal within 24 hours after the start of reperfusion. There is no evidence that a compensatory mechanism induces supranormal adenine nucleotide levels 
following ischemia. These data therefore suggest that in contrast to what is generally accepted in heart tissue, ischemic renal cells may loose enzyme activity without being irreversibly damaged.

Chapter 14 describes the effect of cytokines on renal tubular cells in vitro. Cytokines are involved in inflammatory processes and may induce killing of tumour cells and microorganisms. It is shown that IL1 and IFN- $\gamma$ exert cytotoxic properties on normal kidney cells in vitro. The combined action of these cytokines induce cytolysis. Such mechanisms may play a rolle in ischemia and rejection associated inflammation.

Chapter 15 describes the interaction of inflammation and ischemic renal injury in rat. Stimulation of inflammatory reactions with LPS exacerbates renal failure following renal ischemia. The combination of renal ischemia and the stimulation of inflammatory reactions also have a generalized effect as fifty percent of the animals die. In animals without renal ischemia LPS induces neither renal failure nor mortality. Treatment with LPS causes an increase in circulating levels of TNF. Treatment of animals with human recombinant TNF instead of LPS following renal ischemia induces similar renal and generalized complications as in LPS treated animals. TNF may play a role in ischemia-inflammation associated injury as in "reperfusion injury", gramnegative sepsis, and multiple organ failure. Furthermore, these data suggest a new, important field of interest for those looking for better and more rational preservation techniques. 


\section{SAMENVATTING}

Het huidige tekort aan donor nieren zou deels kunnen worden ondervangen door ook nieren van donoren met een circulatie stilstand te gebruiken. Het mogelijke ontstaan van ischemische schade met een onbekende ernst in nieren van dergelijke donoren, staat grootschalig gebruik van deze nieren in de dagelijkse praktijk in de weg. In dit proefschrift zijn een aantal experimentele studies gebundeld met als thema de betekenis van ischemische schade voor de viabiliteit van de donor nier en de wijze waarop de omvang van de ischemische schade zou kunnen worden gemeten. Dit thema werd vanuit een drietal invalshoeken benaderd, te weten het cellulaire energie metabolisme, enzym velies uit ischemisch beschadigde cellen, en de rol van inflammatoire processen bij het ontstaan van schade door ischemie.

In deel I van van dit boek worden in respectievelijk hoofstuk 1,2 en 3, de resultaten uit het experimentele werk beproken in het licht van deze drie invalshoeken en tegen de achtergrond van resultaten uit onderzoek van anderen.

In deel II, hoofdstuk 4 tot en met 15, worden experimentele studies afzonderlijk weergegeven in de vorm waarin ze zijn gepubliceerd of ter publicatie zijn aangeboden.

Hoofdstuk 4 beschrijft een methode om de warme ischemie tijd in donor nieren vast te stellen. Uit studies in het hondemodel blijkt de ratio van de adenine nucleotide concentratie en de concentratie van afbraakproducten in nierweefsel te corresponderen met de duur van de ischemie. Veranderingen in temperatuur dienen betrokken te worden in de interpretatie van deze ratio. Wat betreft mogelijke klinische toepassing is het van belang dat de genoemde metabolieten kunnen worden bepaald in eenvoudig uit de nier te nemen corticale wigbiopten.

Hoofdstuk 5 is een studie over het effect van hypothermie op de afbraak van adenine nucleotiden in honde nieren tijdens ischemie. De daling van de adenine nucleotide concentratie is sterker naarmate de concentratie voor het begin van de hypothermie hoger is. $\mathrm{Na} 72$ uur koude preservatie $\left(4^{\circ} \mathrm{C}\right)$ wordt in alle nieren een zelfde adenine nucleotide concentratie bereikt ongeacht het effect van de beginconcentratie. Veranderingen van de beginconcentratie door warme ischemie zijn daardoor moeilijk vast te stellen aan de hand van de adenine nucleotide concentratie tijdens hypothermie. Hoofdstuk 6 beschrijft in een studie in het het rattemodel, de effectiviteit wan totale lichaamskoeling als methode om koude preservatie te starten. Het verlagen van de lichaamstemperatuur van $37^{\circ} \mathrm{C}$ tot $9.2^{\circ} \mathrm{C}$ binnen een uur geeft een reële vermindering van het adenine nucleotide verlies dat optreedt in nieren na het intreden van een circulatie stillstand. Met een dergelijke eenvoudige en niet invasieve methode om orgaan preservatie te starten zouden technische en juridische problemen rond potentiele non-heartbeating donoren kunnen worden vermeden.

Hoofdstuk 7 beschrijft het herstel van het adenine nucleotide metabolisme tijdens herstel van de circulatie na voorafgaande preservatie en warme ischemie. Zonder preservatie neemt de adenine nucleotide concentratie tijdens een uur in situ perfusie toe afhankelijk van de warme ischemie tijd. $\mathrm{Na} 48$ uur preservatie is een dergelijke relatie niet aantoonbaar. Er is geen toename van de adenine nucleotide concentratie binnen een uur. Het vermogen om ATP te resynthetiseren lijkt in alle gevallen behouden. Een rol voor de degradatie producten als nucleotide precursor is niet aantoonbaar. Hoofdstuk 8 beschrijft een gemodificeerde. HPLC methode waarmee de concentratie 
van 19 metabolieten waaronder adenine nucleotiden, guanine nucleotiden en een aantal degradatie producten van deze stoffen in nierweefsel kan worden gemeten. Slechts $5 \mathrm{mg}$ weefsel op natgewicht basis is nodig voor een accurate bepaling. Zowel de interals intra-assay variatie coefficient is acceptabel voor de bedoelde toepassing. De concentratie van nucleotiden in corticaal nierweefsel is in humane nieren vergelijkbaar met die in hondenieren.

Hoofdstuk 9 beschrijft de relatie tussen het herstel van het adenine nucleotide metabolisme en de zogenaamde life sustaining functie van nieren na ischemie. Verlenging van de warme ischemie tijd van 30 naar 90 minuten veroorzaakt een sterk verminderde toename van nucleotiden in het eerst uur na reperfusie en het uitblijven van life sustaining functie. Vijf dagen na het aanbrengen van de ischemie is het verschil tussen nieren met 30 en 90 minuten ischemie verdwenen wat betreft de weefsel adenine nucleotide concentratie. Als nieren na 90 minuten ischemie vijf weken de tijd krijgen om te herstellen blijkt ook de life sustaining functie te zijn teruggekeerd. Andere factoren dan alleen het adenine nucleotide metabolisme zijn waarschijnlijk verantwoordelijk voor dit trage functie herstel.

Hoofdstuk 10 beschrijft de adenine nucleotide homeostase en de life sustaining functie van nieren na warme ischemie en preservatie met of zonder een kortdurende perfusie van de nier met bloed bij lichaamstemperatuur halverwege de preservatie periode. De tussentijdse perfusie vermindert het adenine nucleotide verlies tijdens preservatie en lijkt de life sustaining functie te verbeteren. Daarnaast wordt beschreven hoe de spoel procedure met Eurocollins aan het begin van de preservatie een sterker verlies van adenine nucleotiden veroorzaakt dan op basis van alleen de ischemie duur en de temperatuursverlaging tijdens deze procedure, zou mogen worden verwacht.

Hoofdstuk 11 beschrijft de transportweg van enzymen die buiten de cel zijn geraakt. Enzymen die door middel van infusies in nier parenchym zijn gebracht worden snel en vrijwel compleet in het bloed aangetroffen en niet in de urine. Ook in ischemisch beschadigd weefsel volgen geinfundeerde enzymen deze weg. Overeenkomstig deze bevindingen blijken na nier ischemie, in quantitatief opzicht, wel belangrijke veranderingen in de enzym activiteit in plasma op te treden maar niet in de urine. De quantitatieve bepaling van cytosolische enzym verlies na nier ischemie dient daarom uit te gaan van veranderingen in de plasma enzym activiteit.

Hoofdstuk 12 beschrijft de relatie tussen enzym verlies uit de nier na ischemie enerzijds en de veranderingen in plasma enzym activiteit in dezelfde tijd anderzijds. De totale hoeveelheid enzym activiteit die gedurende een bepaalde periode in het bloed verschijnt kan worden berekend aan de hand van een twee-compartimenten model voor circulerende eiwitten. De totale hoeveelheid AST die gedurende 48 uur na ischemie in het bloed accumuleert komt overeen met ongeveer $90 \%$ van het verlies aan AST uit het weefsel in dezelfde periode. Het enzym verlies uit ischemisch nier weefsel lijkt goed te kunnen worden berekend aan de hand van veranderingen in de plasma enzym activiteit. Deze berekening vergt het frequent nemen van bloedmonsters gedurende vooral de eerste 24 uur. Verschillen in enzym verlies in mieren met permanente ischemie en in nieren met ischemie en reperfusie, suggereren dat tijdens reperfusie een additionele schade optreedt.

Hoofstuk 13 beschrijft de relatie tussen enzym verlies uit nierweefsel en het optreden van irreversibele cel schade. De weefsel enzym activiteit in de nier na 90 minuten 
ischemie neemt in een daaropvolgende periode van 48 uur met ongeveer $50 \%$ af. De adenine nucleotide concentratie daarentegen, is aanvankelijk verlaagd maar na 24 uur alweer bijna terug op een pre-ischemisch niveau. Controle experimenten maken het onwaarschijnlijk dat een compensatie mechanisme supra-normale adenine nucleotide concentraties veroorzaakt in een deel van het nierweefsel. Daarom lijkt de conclusie gerechtvaardigd dat ischemische niercellen enzymen kunnen verliezen zonder irreversibel beschadigd te zijn.

Hoofdstuk 14 beschrijft het directe effect van een tweetal cytokines op renale tubulus epitheel cellen in vitro. Cytokines spelen een rol in ontstekings processen en kunnen verantwoordelijk zijn voor de vernietiging van tumorcellen en micro-organismen. IL1 en IFN- $y$ blijken ook cytotoxisch te zijn voor normale nier parenchym cellen. De combinatie van IL1 en IFN-y veroorzaakt cytolyse van deze cellen. Mogelijk spelen deze eigenschappen van de cytokines een rol in het uiteindelijke effect van ontstekingen die optreden tijdens ischemie en rejectie.

Hoofdstuk 15 tenslotte beschrijft de wisselwerking tussen ischemische schade en ontstekings processen na nier ischemie in de rat. Stimulatie van ontstekings processen met LPS verergert het nierfunctie verlies na nier ischemie. De combinatie van nier ischemie en LPS behandeling leidt ook tot een gegeneraliseerd effect zoals blijkt uit een mortaliteit van 50\%. In dieren zonder nier ischemie leidt LPS noch tot nierfunctie verlies noch tot mortaliteit. LPS veroorzaakt een stijging van de TNF plasmaspiegel. Behandeling van dieren met humaan recombinant TNF in plaats van LPS, leidt in combinatie met nier ischemie tot dezelfde nierafwijkingen en gegeneraliseerde complicaties als behandeling met LPS. De suggestie wordt daarmee gewekt dat endogeen TNF een belangrijke rol speelt in schade door ontstekingsprocessen in combinatie met ischemie. Deze experimentele gegevens zijn niet alleen van belang in het licht van klinische problemen als gram negatieve sepsis en multiple organ failure maar geven ook inzicht in de implicatie op termijn van ischemische schade in donor nieren en suggereren een belangrijk nieuw aandachtsveld binnen het onderzoek naar betere preservatie methoden. 


\section{DANKWOORD}

Het tot stand komen van een "boekje" is voor velen een last, voor slechts één enkeling een lust. Ik ben dan ook veel dank verschuldigd aan allen die tot de eerste categorie behoren.

Op de allereerste plaats aan Prof.Dr.G.Kootstra die me geleerd heeft hoe je de boom in moet klimmen, gelukkig zal hij ook les 2 voor zijn rekening nemen. Prof.Dr. G.J. van der Vusse, Dr. W.T.H. Hermens en Dr. W.A. Buurman hebben zich veel moeite getroost mij, ofschoon op zeer verschillende wijze, hetzelfde wetenschappelijke metier te onderwijzen. Met Michael Vork als running mate genoot ik een rugdekking die me de gelegenheid bood breed en diep te gaan. Het dagelijkse leven in de afgelopen drie jaar speelde zich voornamelijk af binnen de groene muren van de operatie kamer waar May Bost zorg droeg voor alle denkbare technische en huishoudelijke bijstand en aldus de moed erin hield. Kamer en lotgenoot Charles van Berlo nam steed breeduit de tijd om me mijn leed met zijn leed te doen vergeten.

Ideeën blijken vooral in discussies te worden geboren en zijn daardoor moeilijk aan ến iemand toe te schrijven. Daarom dank aan George Willems, Karel Leunissen, Marc van Bilzen, Henny Peltenburg, Eric van Veen, Frits Prinzen, Kees Ruissen, Jan Willem Greve, Bert Rijkmans, Luc Snoeckx en Math Daemen.

Het werken met grote en kleine proefdieren vergt veel hulp. Jet Beekman, Ruud Kruger, Peter Franssen, Frans Slangen, Petra Rommers, Peter Kelderman, Will Coumans, Huub Simons, Frans Weekers, Jo Habets, Ton van den Bogaard, medewerkers van de instrumentele dienst, Johan van Dongen, Peter van de Klundert en niet te vergeten de vele assistentes van May Bost, Lotte, Piep, Mieke, Doortje, en Joyce, Bedankt!

De verwerking van gegevens is aan het begin van de vele ontwikkelingen op het gebied van de automatisering mischien sneller maar nog zeker niet eenvoudiger geworden. Tussen potlood en laserprinter kreeg ik hulp van Karin Spronck, Marianne Sieb, Charles van Berlo, Theo van de Nagel, Rob Maessen, Henk Jas en Jac Beursgens.

De leden van de beoordelingscommissie, Prof.P.J. Hoedemaeker, Dr.J.P. van Hooff, Dr. J.W. de Jong, Prof.Dr.R.S. Reneman, Dr.M.J.H. Slooff, Prof.Dr.H.A.J. Struyker Boudier, en Prof.Dr.O.C.K.M. Penn als voorzitter, wil ik bedanken voor de opbouwende kri. tiek bij het nemen van de laatste hindernis.

Mijn ouders zijn nog steeds mijn trouwste supporters ongeacht hetgeen in het veld gebeurt.

Jet, jouw gezond relativeringsvermogen heeft me voor veel dingen behoed, met name voor een lijviger boekwerk. Laat die volgende projecten maar komen. 


\section{CURRICULUM VITAE}

Josephus Gregorius Maessen werd geboren op 19 februari 1960 te Roermond. Hij behaalde in 1978 het eindexamen gymnasium $\beta$ aan het Stedelijk Lyceum te Maastricht. Hierna begon hij de studie Geneeskunde aan de Rijksuniversiteit Limburg en behaalde in 1984 het arts examen. Sindsdien is hij werkzaam als wetenschappelijk assistent bij de vakgroep Algemene Heelkunde (Prof. Dr. J.M. Greep). 